\title{
Trust, Accountability
} and Capacity in Education System Reform

Global Perspectives in Comparative Education

\author{
EDITED BY
}

MELANIE EHREN AND JACQUELINE BAXTER

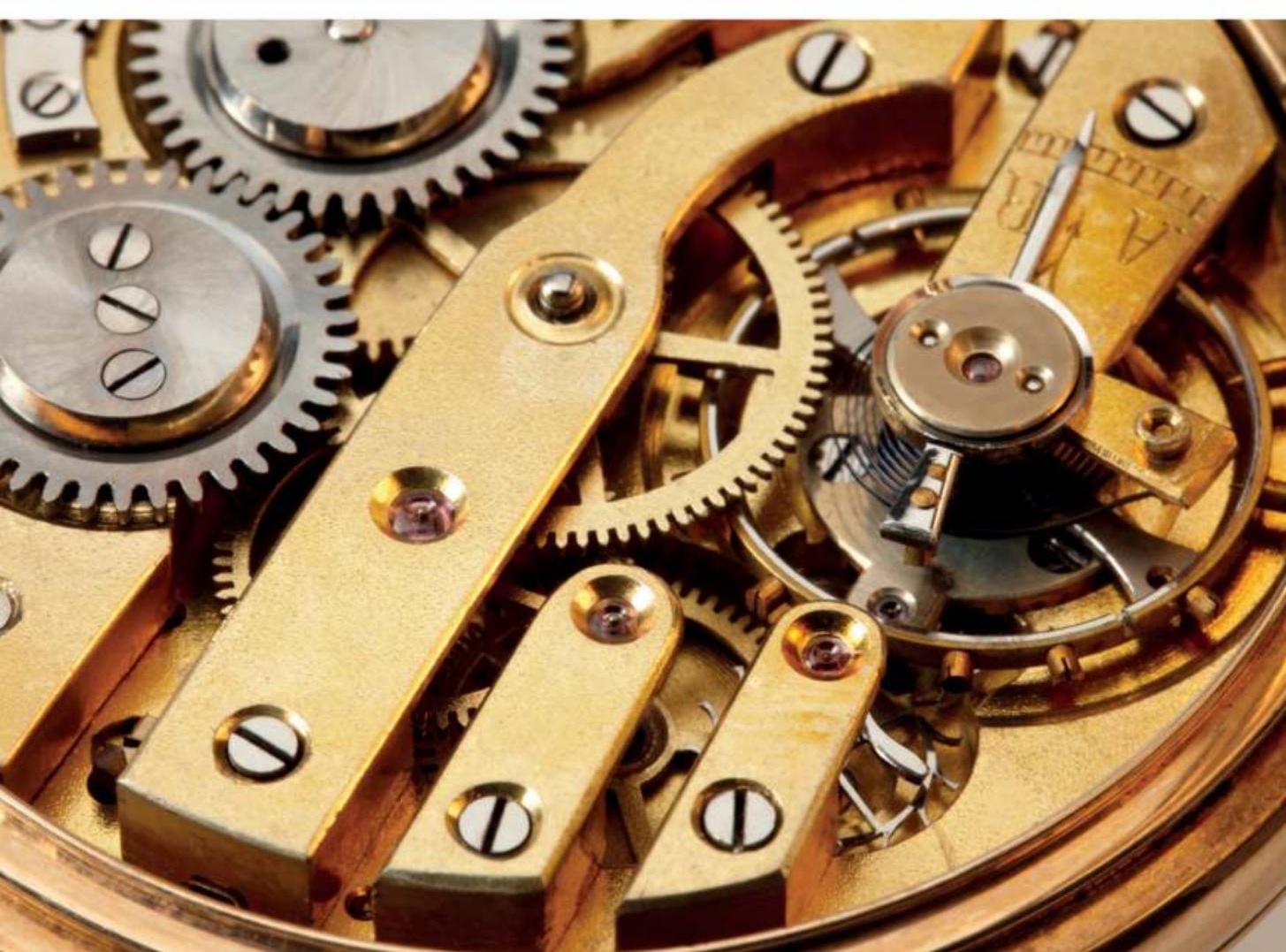




\section{TRUST, ACCOUNTABILITY AND CAPACITY IN EDUCATION SYSTEM REFORM}

This global collection brings a new perspective to the field of comparative education by presenting trust, capacity and accountability as the three building blocks of education systems and education system reform. In exploring how these three factors relate to student learning outcomes across different international contexts, this book provides a powerful framework for a more equal system.

Drawing upon research and case studies from scholars, policymakers and experts from international agencies across five continents, this book shows how trust, capacity and accountability interact in ways and with consequences that vary among countries, pointing readers towards understanding potential leverage points for system change.

Trust, Accountability and Capacity in Education System Reform illuminates how these three concepts are embedded in an institutional context temporally, socially and institutionally and offers an analysis that will be of use to researchers, policymakers and agencies working in comparative education and towards education system reform.

Melanie Ehren is a professor in educational governance and the Director of research institute LEARN! at the Vrije Universiteit Amsterdam and an honorary professor at the UCL Institute of Education, UK.

Jacqueline Baxter is an associate professor in Public Policy and Management and the Director of the Centre for Research and Innovation in Online Business and Legal Education at The Open University, UK. 
$\Longrightarrow$ Taylor \& Francis Taylor \& Francis Group

http://taylorandfrancis.com 


\section{TRUST, \\ ACCOUNTABILITY \\ AND CAPACITY IN \\ EDUCATION SYSTEM \\ REFORM}

Global Perspectives in

Comparative Education

Edited by Melanie Ehren and Jacqueline Baxter 
First published 2021

by Routledge

2 Park Square, Milton Park, Abingdon, Oxon OX14 4RN

and by Routledge

52 Vanderbilt Avenue, New York, NY 10017

Routledge is an imprint of the Taylor \& Francis Group, an informa business

(C) 2021 selection and editorial matter, Melanie Ehren and Jacqueline

Baxter; individual chapters, the contributors

The right of Melanie Ehren and Jacqueline Baxter to be identified as the authors of the editorial material, and of the authors for their individual chapters, has been asserted in accordance with Sections 77 and 78 of the Copyright, Designs and Patents Act 1988.

All rights reserved. No part of this book may be reprinted or reproduced or utilized in any form or by any electronic, mechanical or other means, now known or hereafter invented, including photocopying and recording, or in any information storage or retrieval system, without permission in writing from the publishers.

Trademark notice: Product or corporate names may be trademarks or registered trademarks and are used only for identification and explanation without intent to infringe.

British Library Cataloguing-in-Publication Data

A catalogue record for this book is available from the British Library

Library of Congress Cataloging-in-Publication Data

A catalog record has been requested for this book

ISBN: 978-0-367-36247-8 (hbk)

ISBN: 978-0-367-36249-2 (pbk)

ISBN: 978-0-429-34485-5 (ebk)

Typeset in Bembo Std

by KnowledgeWorks Global Ltd. 


\section{CONTENTS}

List of contributors

vii

Acknowledgements

Foreword

1 Trust, accountability and capacity: Three building blocks of education system reform

Melanie Ehren and Jacqueline Baxter

2 Governance of education systems: Trust, accountability and capacity in hierarchies, markets and networks

Melanie Ehren and Jacqueline Baxter

3 Trust-based accountability in education:

The role of intrinsic motivation

Frédérique Six

4 Distrusting contexts and cultures and capacity

for system-level improvement

Jacqueline Baxter

5 Accountability to build school and system improvement capacity

Melanie Ehren and Reinhard Bachmann

6 Inner group trust and school autonomy in a segregated school system; parental self-segregation in the Netherlands 
vi Contents

7 Trust, professional capacity and accountability in school improvement: Austria's quality management system

Herbert Altrichter

8 Hierarchical structures with networks for accountability and capacity building in Singapore: An evolutionary approach Shu-Shing Lee, Jeanne Ho and Tay Lee Yong

9 Educational technology to improve capacity - integrating adaptive education programmes in public school in Kenya Tom Kaye

10 From hierarchy and market to hierarchy and network governance in Chile: Enhancing accountability, capacity and trust in public education

Carmen Montecinos, Álvaro González, and Melanie Ehren

11 Contrasting approaches, comparable efficacy?:

How macro-level trust influences teacher accountability in Finland and Singapore Yue-Yi Hwa

12 Distrust, accountability and capacity in South Africa's fragmented education system

Nomancotsho Pakade and Thokozani Chilenga-Butao

13 Downward spiral or upward trajectory?:

Building a public profession to meet the shifting technical, social and political demands of education Joshua L. Glazer and Jal Mehta

14 Trust, capacity and accountability: A triptych for improving learning outcomes

Melanie Ehren and Jacqueline Baxter 


\section{LIST OF CONTRIBUTORS}

Herbert Altrichter is a professor of Education and Educational Psychology and the Director of Linz School of Education, Linz School of Education and Johannes Kepler University.

Research interests: Educational governance, school development and system reform, evaluation, teacher education.

Reinhard Bachmann is a professor at SOAS University of London. Reinhard Bachmann teaches and conducts scholarly activities at SOAS University of London, UK. He is a professor of International Management and the Founder and Director of the Centre for Trust Research at SOAS. His research focuses on issues of strategic management, organizational analysis and comparative management. Specifically, the role of social mechanisms (trust, power etc.) and societal influences (institutional arrangements, cultural traditions) on the structure and strategic organization of business relationships is emphasized in his work. Reinhard specifically published seminal work on trust in journals such as Organization Studies, Cambridge Journal of Economics, International Sociology and Journal of Managerial Psychology, and he edited the 'Handbook of Trust Research', 2006, the 'Landmark Papers on Trust', 2008, and the 'Handbook of Advances in Trust Research', 2014 (all three volumes with Akbar Zaheer).

Dr Jacqueline Baxter is an associate professor in Public Policy and Management and the Director of the Centre for Research and Innovation in Online Business and Legal Education at The Open University, UK. Her research interests lie in the area of public policy, accountability and governance. She is a fellow of the Academy of Social Sciences and the Editor-in-Chief of the Sage Journal: Management in Education. 
Thokozani Chilenga-Butao is an associate lecturer, Department of Political Studies, University of the Witwatersrand, South Africa. Her research interests include decentralization, public administration, public policy and the governance of education. Her PhD focuses on national government interventions in South Africa's provincial education departments. Her ongoing research examines South Africa's national education department and its decentralized relationship with provinces and schools.

Melanie Ehren is a professor in educational governance and the Director of research institute LEARN! at the Vrije Universiteit Amsterdam and an honorary professor at the UCL Institute of Education. Her academic work focuses on the effectiveness of accountability and evaluation systems and aims to contribute to a greater understanding of the interplay between accountability and the broader education system in tackling inequality and improving student outcomes.

Joshua L. Glazer is an associate professor of Education Policy at George Washington University. His research and teaching examine multiple approaches to improving underperforming schools in high poverty, urban environments; the political dynamics of state takeovers; and the design of research practice partnerships.

Álvaro González is postdoctoral researcher at the Instituto de Ciencias de la Educación of the Universidad de O'Higgins, Chile. His academic work focuses on educational improvement and change, addressing topics such as educational leadership, professional and organizational learning, accountability and evaluation and education policy.

Jeanne Ho is a teaching fellow at the Office of Education Research - National Institute of Education, Nanyang Technological University. She has taught in primary schools for more than two decades. His research interests are in the field of ICT in education, metacognition and teacher professional development.

Yue-Yi Hwa is a research fellow at the Research on Improving Systems of Education (RISE) Programme, based at the Blavatnik School of Government, University of Oxford. The research in this chapter was conducted as part of her $\mathrm{PhD}$ at the University of Cambridge on teacher accountability and sociocultural context across countries.

Tom Kaye is an education consultant with the World Bank Group who focuses on implementing EdTech to enhance learning outcomes in low-performing education systems. He has experience working in countries across Africa, Asia, Australia and the Middle East. 
Shu-Shing Lee is a research scientist at the Centre for Research in Pedagogy and Practice, National Institute of Education, Nanyang Technological University, Singapore. Her research interests include understanding teacher learning from a social-cultural perspective as well as understanding contextual factors and leverages that influence teacher learning.

Jal Mehta is a professor of Education at the Harvard Graduate School of Education. His work focuses on how to create schools and systems that enable all students and adults to thrive, with a particular interest in the professionalization of teaching. He is the author, most recently, with Sarah Fine, of In Search of Deeper Learning: The Quest to Remake the American High School and The Allure of Order: High Hopes, Dashed Expectations, and the Troubled Quest to Remake American Schooling. He is the proud recipient of the Morningstar Award for Teaching Excellence at the Harvard Graduate School of Education.

Carmen Montecinos is a professor in Educational Psychology and the Executive Director of the Educational Leadership Centre PUCV at Pontificia Universidad Católica de Valparaiso in Chile. She is a principal investigator with the Centre for Advanced Research in Education at Universidad de Chile focusing on policies and practices around school practitioners' workplace configurations and learning and their impact on school outcomes.

Nomancotsho Pakade has extensive working experience in community mobilization and advocacy within the NGO sector. She has been involved in research projects focusing on health and education. She holds an MA in Research Psychology from the University of Witwatersrand. Currently, Noma is a researcher at the Public Affairs Research Institution (PARI).

Frédérique Six is an associate professor for Public Governance at the Vrije Universiteit Amsterdam. Her research focuses on public governance and in particular the relationship between trust and control/accountability, applying this to how professionals are governed and to regulation.

Tay Lee Yong is a teaching fellow at the Office of Education Research - National Institute of Education, Nanyang Technological University. Tay Lee Yong has taught in primary schools for more than two decades. His research interests are in the field of ICT in education, metacognition and teacher professional development. 


\section{ACKNOWLEDGEMENTS}

The Editors gratefully acknowledge funding from the Department for International Development, under the programme: Accountability, capacity and trust to improve learning outcomes in South Africa: a systems approach. Grant reference number: ES/POO5888/1. Fund start date: September 01 2017: Duration: 4 years. (https://www.jet.org.za/work/project-showcase/accountabilityin-education/accountability-capacity-and-trust-to-improve-learning-outcomesin-south-africa-a-systems-approach). 


\title{
FOREWORD
}

\author{
Andreas Schleicher
}

Changing school systems can be like moving graveyards: it is often hard to rely on the people out there to help, because the status quo has so many protectors. Meanwhile, the changes in our societies have vastly outpaced the structural capacity of our school systems to respond. Even the best education minister can no longer do justice to the needs of millions of students, hundreds of thousands of teachers and tens of thousands of schools. The challenge is to build on the expertise of teachers and school leaders and enlist them in the design of superior policies and practices. This is not accomplished just by letting a thousand flowers bloom; it requires a carefully crafted enabling environment around capacity, trust and accountability that can unleash teachers' and schools' ingenuity and build capacity for change.

Leaders who want to make forward-looking changes in their schools and school systems need to build a shared understanding and collective ownership, make the case for change, and offer support that will make change a reality. They need to focus resources, build capacity, change work organisations, and create the right policy climate with accountability measures designed to encourage innovation and development, rather than compliance.

That relies on trust: trust in institutions, in educators and in students. Without trust, support for change is difficult to mobilise, particularly where short-term sacrifices are involved and long-term benefits not immediately evident. Low trust can also lead to lower rates of compliance, and in turn to more stringent and bureaucratic regulations or administrative accountability. In all public services, trust is an essential part of good governance. Successful schools will always be places where people want to work, where their ideas can be best realised, where they are trusted and in which they can place their trust. 
We know still little about how trust is developed in education and sustained over time, or how it can be restored if broken. But trust cannot be legislated or mandated; that is why it is so hard to build into traditional administrative structures. Trust is always intentional; it can only be nurtured and inspired through healthy relationships and constructive transparency. At a time when commandand-control systems are weakening, building trust is essential to advance and fuel modern education systems.

Some people say one cannot give teachers and education leaders greater autonomy because they lack the capacity and expertise to deliver on it. There may be some truth in that. But simply perpetuating a prescriptive model of teaching will not produce creative teachers: those trained only to reheat pre-cooked hamburgers are unlikely to become master chefs. It is when teachers feel a sense of ownership over their classrooms and when students feel a sense of ownership over their learning that productive teaching takes place. That is the fundamental problem of systems where administrative accountability arrangements stifle autonomy, they do not generate and sustain capacity. So the answer is to strengthen trust, transparency, professional autonomy and the collaborative culture of the profession all at the same time.

Much has been written about trust, accountability and capacity in education. What Trust, Accountability, and Capacity in Education System Reform adds to the literature, beyond a comprehensive analysis of these concepts, is a systematic and insightful discussion of the interrelationships between these concepts. And it is the understanding of those interrelationships that will help policy-makers and practitioners address the some of the most persistent challenges in education: whether these concern the lack of professional agency of teachers, overburdening bureaucracy or the loss of legitimacy of public education. 


\title{
TRUST, ACCOUNTABILITY AND CAPACITY
}

\section{Three building blocks of education system reform}

\author{
Melanie Ehren and Jacqueline Baxter
}

\section{Introduction: A preoccupation with system change}

Almost every country has undertaken some form of school system reform during the past two decades, trying to improve student outcomes and creating more equal systems. The quality of education is seen as one of the most important elements to the well-being of a country and its competitive advantage over other countries. International league tables (such as PISA or TIMSS) have sparked debates about the 'best' systems across the world where policymakers try and learn about successful reform strategies in their countries and how to replicate their success. However, systems are inherently complex; they have necessarily disparate goals and face different challenges as starting points for change vary as well as the socio-economic, cultural and political context of change.

Over the years, various authors in the field of comparative education have developed frameworks to understand education systems. Points of comparison have been as varied as looking at educational achievements, various policies, curricula, values and locus of decision-making where the country or nation-state has had particular prominence (Bray et al., 2014). International league tables, such as the OECD's PISA, emphasise academic outcomes and have generated great interest among policymakers and researchers alike to understand the high performance of countries such as Finland and Singapore. 'What works' and the search for good practices underpin much of the interest in comparative education and many international agencies have long-term studies and projects to provide such insights. Examples are the OECD's 'governing complex systems' project which has produced various working papers to, amongst others, understand accountability, knowledge governance and stakeholder involvement in education systems; the World Bank's SABER initiative has produced comparative data and knowledge on education policies and institutions in over 100 countries on 
policies as varied as early childhood development, student assessment and teacher policy to equity and inclusion, tertiary education and skills development, while UNESCO's annual Global Education Monitoring Report presents progress of 209 countries and territories towards the education targets in the sustainable development goals.

These studies have particularly produced cross-sectional data about a range of indicators and countries. Other studies, such as Mourshed et al. (2010) also aim to understand successful trajectories of system change. In their report, Mourshed et al. (2010) present clusters of interventions for systems that are either poor, fair, good or great, trying to outline a path for improving school system performance. The report has been highly influential in shaping policymakers' thinking about stages of development, but the report was also criticised for its simplicity. Coffield (2012) for example critiqued the lack of appreciation for the dynamic nature of change and the fact that the linear presentation of stages of change does not acknowledge the influence of history, culture, values, existing policies and structures, each of which will affect the success of reforms in different ways. Most of the comparative work, both in the academic field as by international agencies focuses on educational contexts, inputs and outputs, paying relatively little attention to indicators that help guide improvement in education governance of nation states, and how policy and reform processes are best realised, given the context in which they are introduced (Burns, 2012).

This book aims to understand education system performance and change through the lens of trust, accountability and capacity. The three variables and their interaction allow us to understand education systems from the perspective of inter-stakeholder relations (across the locational levels and for various demographic groups) and how these are situated both temporally as well as within the wider socio-economic, cultural and political context. We consider trust, capacity and accountability as the building blocks of any education system, and their interaction over time allows us to explain some of the possibilities and constraints for successful reform and how these may vary across countries. Trust for example allows us to explain the interactions between stakeholders such as policymakers, school leaders, teachers and parents involved in providing high-quality education and why collaboration between some stakeholders is more successful in building capacity for improvement. Capacity of stakeholders (their knowledge, skills and decision-making power) is needed to provide a high standard of education and deliver on their (new) responsibilities (once agreed on), while accountability provides a set of incentives for improvement, as well as information to understand where improvement is needed.

Trust, capacity and accountability are frequently studied and discussed separately in the field of political science, publication administration and education research on school effectiveness and improvement. There is however little discussion on how the three are related, both on the school level and on the system/policy/governance level. Throughout the book we will argue that it is particularly the interaction between trust, accountability and capacity that helps 
us understand performance of education systems and identify levers for change. Accountability and trust are for example considered beneficial for schools and systems to have or build the capacity for change, but both are at odds with one another where the direction of the relationship between trust and accountability is complex and bidirectional: accountability often destroys trust when governments introduce high-stakes external control in a context of distrust in schools and teachers. This phenomenon of 'crowding out' (see Gundlach and Cannon, 2010; Puranam and Vanneste, 2009) is well exemplified in South Africa where teachers and head teachers view eternal monitoring through their memories of the Apartheid regime where control equalled suppression instead of support for improvement.

Accountability may however also improve trust when locking partners into a sustainable collaboration and setting a clear set of performance standards that regulate their interaction. Ehren et al. (2015) work on school inspections in a number of European countries for example showed how inspection frameworks and standards created a common language for improvement and set of goals to word towards. Capacity, our third variable of interest, can be seen as an outcome of both trust and accountability: when teachers trust each other and collaborate well in developing a coherent curriculum the quality of school and student outcomes improve. Similarly, accountability, through the provision of performance feedback, is expected to improve schools and student learning, and many of such systems particularly aim to do so.

Any time a reform is rolled out, we thus need to think carefully about the available capacity, how people and organisations are held accountable and how actors in the system (students, parents, teachers, principals, school boards, policymakers) trust each other. Successful governance, according to Burns (2012), requires thinking about the individuals involved, their needs and their aspirations and how they interact. Some of the questions she poses are as follows:

'Can parents rely on equal quality of teaching and learning across schools? Do teachers have similar working conditions and rewards? How are organisational stakeholders being included in the decision-making processes? And how worried do stakeholders have to be about corruption in the system? Do teachers (and principals, students and parents) have the capacity to deliver on their new responsibilities? If not, is training or other support in place? Do people and organisations trust each other to collaborate, do principals and teachers trust their government to let them decide on specific levels of implementation or is their agency taken away as soon as something goes wrong?' These all affect the performance of a system and whether reform will happen. It is however these questions that often get lost when governments move forward with a new reform or policy (Burns and Cerna, 2016).

The set of questions to consider when looking at trust, accountability and capacity of schools and education systems are enormous and any attempt to answer them are challenging at best. We therefore start this book by presenting a framework to position trust, accountability and capacity as elements of, 
and processes in education systems that support or hamper learning and development of educators. We'll use Bronfenbrenner's ecological systems theory of human development which situates learning and development of individuals in a microsystem that is embedded in a meso (school)system, and in the institutional structure and social blueprint of an exo and macrosystem, subject to time in the chronosystem. In choosing a framework about human development to understand education systems, we argue that improvement of student learning is ultimately the outcome of how people behave and interact, where the improvement of teaching and learning of students requires a change in behavioural patterns of teachers, school leaders and other stakeholders in the system to more effective ways of working. In Chapter 2, we will present three ideal types of systems and use our triad of trust, accountability and capacity to describe how each system is vastly different in organising inter-stakeholder relations and creating or restricting opportunities for learning and development. Positioning trust, accountability and capacity in relation to system governance allows us to start unpacking the structural and temporal context of system performance and change. First we start by defining what we mean by 'system' and presenting Bronfenbrenner's systems approach to human development.

\section{Defining 'system'}

'Systems', their patterns of behaviour and elements, interconnections and functions or purposes, have been studied extensively in ecological science. Meadows (2008, p. 2) describes a system as 'a set of things - people, cells, molecules, or whatever - interconnected in such a way that they produce their own pattern of behaviour over time'. The most obvious component parts of education systems would be the institutions and individual actors that operate together within a common legal and administrative framework (e.g. on examinations, conditions of service for teachers), according to Bray and Jiang (2014) and Snyder (2013). Through the interactions between individuals, such as teachers, parents, students, principals and policymakers, new structures and behaviours emerge as and when actors act and react to each other, learn and develop over time. The result, according to Snyder (2013), can be qualitatively different from the sum of individual actions and learning, such as when frequent contact between teachers in a school creates social capital and a safe school culture.

The description highlights the embedded nature of relations and how we can think of systems as a set of layers which interact in complex ways, but where the learning of an individual (through the interaction with his/her environment) lies at the heart of any system and system change. This understanding of how learning and development is embedded in a wider context is well described in Bronfenbrenner's (1989) ecological systems theory of human development. He uses a systems' perspective to understand the processes and results of human 
development as a common equation of man and environment, where he described human development as follows:

the process through which the growing person acquires a more extended differentiated, and valid conception of the ecological environment, and becomes motivated and able to engage in activities that reveal the properties of, sustain, or restructure that environment at levels of similar or greater complexity in form and content (Bronfenbrenner, 1989, p. 27; cited in Härkönen, 2005).

The underlying rationale of the theory is that human development results from an interaction of processes, person and context, where developmental processes and outcomes vary as a joint function of the characteristics of the person as well as the environment, and their interactions over the course of time (Bronfenbrenner, 1989, p. 200). The system's perspective conceptualises the learning and development as a set of concentric circles of an individual's microsystem, his/her mesosystem, exosystem, macrosystem and chronosystem as depicted in Figure 1.1.

Various authors, such as Snyder (2013), Johnson (2008) and Godfrey (2014), have used Bronfenbrenner's work to understand school and system improvement, taking the development and learning of the school organisation as a starting point. These authors use the model to understand how organisational developmental processes (e.g. teaching and learning) and outcomes (e.g. student achievement) vary as a joint function of the characteristics of the school and of the ecological systems or environment surrounding the school. Bronfenbrenner's work however sees one person's microsystem as a unique model where a more general model for the learning and development of a group of individuals at the same time would assume commonality in their interactions with their environment.

The microsystem in Bronfenbrenner's model constitutes the interactions at the interpersonal level, such as the actions and interactions of school leaders, teachers, staff, parents, governors and students. The microsystem is the system closest to the developing person and the one in which they have direct contact. A microsystem of a child typically includes family, peers or caregivers, and, in a school setting, his/her teacher(s), other adults working in the school and his/her peers. Extending the theory to the development and learning of adults in a school setting situates teachers, head teachers and even policymakers in the microsystem and how they interact with and learn from others (colleagues, peers, employees, employers, stakeholders). Relationships in a microsystem are bidirectional. In other words, someone's reactions to the people in his/her microsystem will affect how they treat you in return. This is the most influential level of the ecological systems theory, according to Härkönen (2005).

The mesosystem consists of the interactions between the different parts of a person's microsystem, where a person's individual microsystems do not function independently, but are interconnected and assert influence upon one another. 


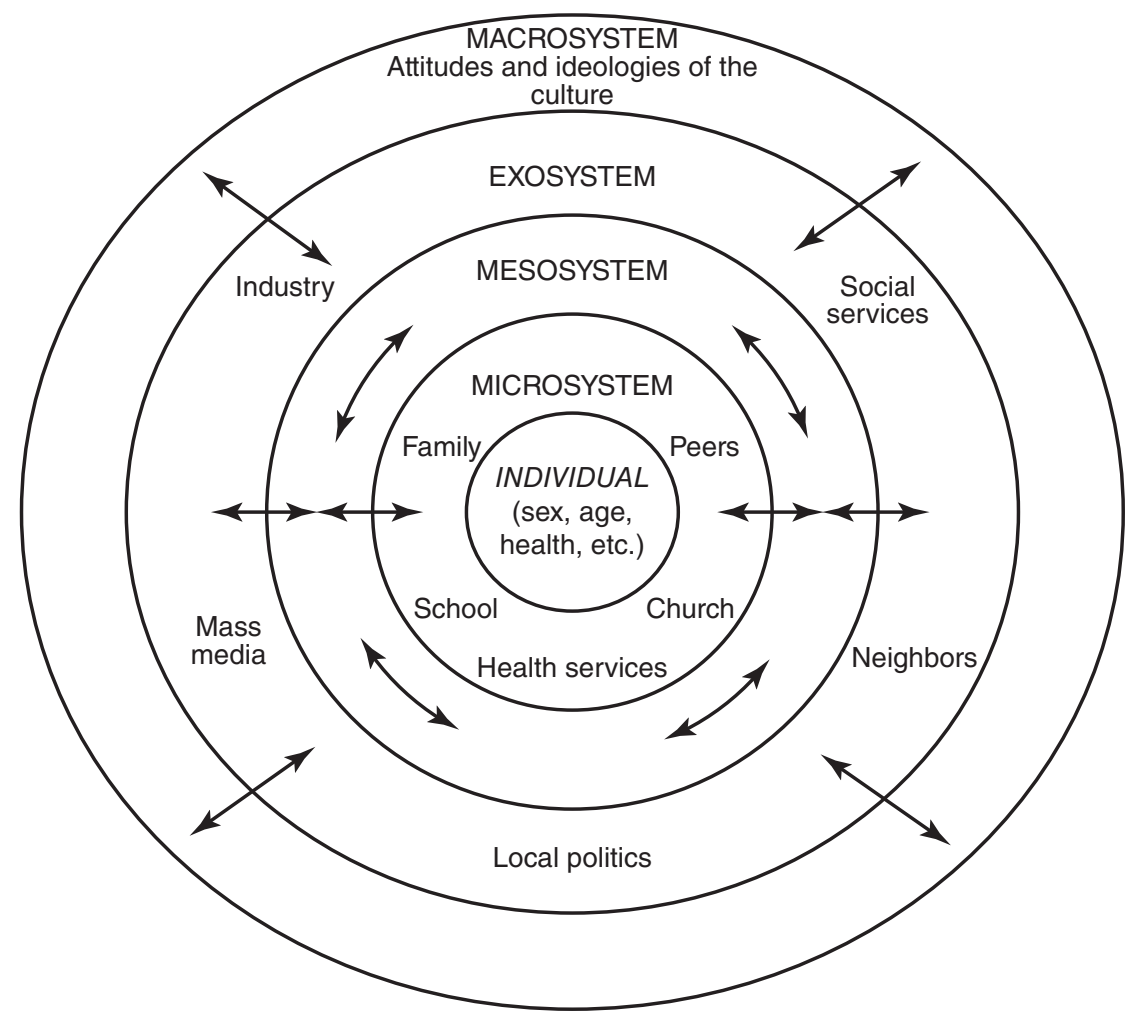

FIGURE 1.1 Bronfenbrenner's (1989) ecological systems theory of human development Source ${ }^{1}$ : Vizcarrondo Oppenheimer, Vélez-Agosto, N. M., Soto-Crespo, J. G., VizcarrondoOppenheimer, M., Vega-Molina, S., \& García Coll, C. (2017). Bronfenbrenner's bioecological theory revision: Moving culture from the macro into the micro. Perspectives on Psychological Science, 12(5), 900-910 (Vélez-Agosto et al., 2017)

These interactions have an indirect impact on the individual through the 'workings' of a school as an organisation or institution (Godfrey and Handscomb, 2019). One aspect of a students' mesosystem would for example be the relationship between the students' parents and his/her teacher. Parents take an active role in his/her school, such as attending parent/teacher conferences and volunteering in his classroom. This has a positive impact on his development because the different elements of his microsystem are working together. The students' development could be affected in a negative way if the different elements of his microsystem were working against one another. A similar argument applies to the learning of, for example, teachers and principals in a school where bidirectional interactions between groups of teachers and students or faculty and parents shape the organisational culture of the school, and for example opportunities to discuss personal problems and quality of work, and thereby influence the learning of those involved. 
The exosystem is the external environment in which individuals act and which exerts influence upon them, such as government policies, networks or other organisations that connect to the school. The exosystem refers to a setting that does not involve the person as an active participant, but still affects them. This includes decisions that have bearing on the person, but in which they have no participation in the decision-making process. An example would be a child being affected by a parent receiving a promotion at work or losing their job. If we take teachers and head teachers as the unit of analysis, examples are elements of the local community, or government policies to strengthen school autonomy and accountability, restraints on public spending, parental demands for choice, state regulations, local economics, federal mandates and local disasters that affect their work and opportunities to learn and develop (Johnson, 2008). Commonplace in many nations' education policies has been the promotion of school-led improvement, coupled with the encouragement of new types of networks of schools, according to Greany and Higham (2018). Such policies emphasise horizontal connections in the ecosystem, specifically at the meso- and exo-levels, through school-to-school collaboration or teachers and school leaders working across schools (Godfrey and Handscomb, 2019), and these will create new relationships and opportunities for learning.

The macrosystem can be thought of as the 'social blueprint' of a given culture, subculture or broad social context and consists of the overarching pattern of values, belief systems, lifestyles, opportunities, customs, and resources embedded therein, according to Johnson (2008). This system is generally considered to exert a unidirectional influence upon not only the person but also his/her micro-, meso-, and exosystem. A child, his or her parent, a teacher, head teacher and his or her school are all part of a large cultural context. Members of a cultural group share a common identity, heritage and values, such as on the purpose of education and reform, and the underlying cultural blueprint in which the system exists. For any particular value system to have an influence on a developing person, it has to be experienced within one or more of the microsystems in which that person is situated (Tudge et al., 2009). This means that students or teachers (the individual we expect to learn and develop) will only be affected by his/her cultural, political, social or economic context if it permeates his/her (opportunities of) interactions with others, access to resources etc. An example is provided by Godfrey (2014) who points to the neoliberal economic and political agenda in England which has dominated the reform agenda in for example setting academic performance targets for schools which will affect how students and school staff interact. The macrosystem evolves over time, because each successive generation may change it.

Such changes are part of what Bronfenbrenner calls the 'chronosystem'. The chronosystem refers to the patterning of environmental events and transitions over the life course, as well as sociohistorical circumstances. Although not one of the four system layers per se, the chronosystem represents a time-based dimension that influences the operation of all levels of the ecological systems, 
according to Johnson (2008). Time can have various short to long-term dimensions (e.g. occurrence of specific activities or activities or patterns of activities), where Bronfenbrenner's work particularly refers to age-related developmental processes of individuals. Tudge et al. (2009) talk about the engines of a child's development, such as parents playing with a child or his/her interaction with peers and learning materials, which change over time when a child grows older, his/her environment changes (including the school context) and interactions with the environment change. When applied to the development and learning of educators working in the school, the experience of teaching and number of years working in the school are relevant age-related time factors that influence learning and development. However, organisational dimensions and how schools and their environment change over time will also have an effect on school staff's learning and development, and of students in schools. Johnson (2008) for example refers to the day-to-day and year-to-year developmental changes that occur in a school's student body, teaching staff, curricular choices and the overall number of years a school is in operation. Newer schools face challenges and opportunities that differ from those of a school that has been in operation for a length of time, and this will affect opportunities of staff working in the school to learn and develop.

Viewed as an ecosystem, the layers in Figure 1.1 are viewed as a set of nested structures where the higher and lower levels mutually influence and shape each other; any change, disturbance or action that occurs at any level can have a disrupting effect on other parts of the ecosystem, including laterally and upwards. Recognising and understanding the mutual relationships across the levels is indispensable for a holistic comprehension of the essence of education systems and how trust, accountability and capacity operate across these layers and influence how students learn and how educators improve their work to enhance their learning. An example comes from England and how the country's continued emphasis on school accountability can for example be explained by its neoliberal political agenda (macrosystem) that highlights and regulates parental choice, competition, quality assurance and performance management (exosystem) and how this impacts on the mesosystem by creating a disproportionate pressure on school leaders to 'account for' their pupils' academic achievements and to find quick fixes where standards are lower than national benchmarks (Godfrey, 2014). The focus on accountability informs interactions between teachers and students, and students and principals in learning how to organise teaching around tested content (microsystem) and inspection standards. Perryman (2006) explains how such high-stakes accountability creates a culture of constant monitoring and performativity, as many heads only want to focus on issues that they anticipated Ofsted, the English Inspectorate of Education, would be interested in. Research has demonstrated a strong influence of accountability testing upon both curriculum organisation and teaching behaviour (e.g. Mansell and James, 2009), identifying the increased prevalence of the use of dubious test preparation strategies, such as 'teaching to the test', over-rehearsal and focusing undue effort 
upon borderline students (Bew, 2011). An Ofsted (2012) report for example describes how schools provided revision or booster classes for pupils in years 6, 9 and 11 and focused particularly on those pupils at risk of narrowly missing the key threshold targets, thereby restricting learning opportunities for those on the lower and higher end of the performance spectrum.

\section{Trust, accountability and capacity on the micro, meso, exo and macro-level}

Modelling education systems using ecological approaches is advocated by Snyder (2013) as a useful alternative to the linear models that often form the basis of educational research and policy. The model acknowledges the complexity of systems and, when applied to education, allows us to describe how trust, accountability and capacity inform learning and development of individuals on the microlevel through their interactions with others, and how trust, accountability and capacity on the meso-level, and in the macro and exosystem influence such learning and development. The chronosystem helps us understand the dynamic nature of change, where we are particularly interested in if and how trust, accountability and capacity reinforce one another over time and enhance or restrict learning and development across the layers in the system. We use a somewhat restricted approach of defining system performance as the aggregated academic outcomes of all learners in public compulsory education where change would include reform, capacity-building efforts or accountability interventions to address academic subject areas or specific student groups that need improvement.

Previous school effectiveness studies emphasise the role of teachers and the quality of their teaching in a student's academic attainment (Barber and Mourshed, 2007; Scheerens, 2009). Various meta-analyses (Hopkins et al., 2014; Muijs et al., 2014; Reynolds et al., 2014) for example present 'time and opportunity to learn', 'classroom management', 'structured teaching', 'teaching and learning strategies' and 'feedback and monitoring' as the main teaching-level variables contributing to high student outcomes. Reform strategies thus need to enable and address their work. We therefore position teachers in the centre of our model and how their school (mesosystem) and macro- and exosystem affect the quality of their teaching and learning and development. Our interest is not in their learning and development per se, nor in what constitutes effective teaching as other studies (e.g. Basma and Savage, 2018; Thurlings and den Brok, 2017) have studied and described both areas in detail.

We ask ourselves how trust, accountability and capacity in/of the school and the education system allow teachers to be or become effective. Relevant questions are how the school context and interactions with others shape their work environment and provide feedback on the quality of their teaching, how their teaching is affected by policy and reform programmes and shape their learning and development over time, either through offering formal training opportunities, through on-site joint collaboration with peers, or more implicitly when 
conditions and methods of their work and the cognitive base of their teaching changes. In the next section, we outline trust, accountability and capacity for each of the layers of Bronfenbrenner's model, providing a framework for subsequent chapters to build on.

\section{Microlevel}

On the microlevel, trust, accountability and capacity are situated in interpersonal relations between teachers and others in their professional environment, such as other teachers, parents, their principal or school board. Do trust, accountability and capacity shape these relations in ways that support high-quality teaching and teacher learning and development?

Investigations of trust in interpersonal relations have traditionally been grounded in the work of economists, sociologists and psychologists, examining, for example, economic transactions between buyer and supplier, interactions between employer-employee, or between regulator-regulatee (Bachmann and Zaheer, 2006; Six and Verhoest, 2017). These fields have aimed to understand trust in various ways, such as a rational and calculated processes and how trust is shaped as a result of purposeful decisions and choices available in a given context (economists and sociologists), or as the result of less explicit, routinised, intuitive and habitual actions where trust constitutes a set of beliefs, emotions, intentions and expectations (psychologists; Lyon, 2006, p. 8; Le Gall and Langley, 2015, p. 38).

A common definition of trust across these studies is 'a trustor's willingness to take a risk based on assessments of a trustee's competence, benevolence and integrity' (Mayer et al.'s, 1995; cited in Addison, 2015, p. 156). These three dimensions are further described by Oomsels and Bouckaert (2017, pp. 82-88) where we provide examples for a school context:

- Competence: The perceived ability, or expectation that the other party has competence to successfully complete its task. Parents will for example trust teachers whom they feel are competent to teach specific subjects. A person who means well but does not have the competence cannot be trusted, especially in the case of dependency and the involvement of some level of skill in the fulfilment of an expectation (Cerna, 2014). The roles people fulfil and the expectations and responsibilities that come with that role underpin an assessment of someone's competence. The example Seashore Louis (2007) provides of someone who was highly trusted as a principal but instantly distrusted when promoted to superintendent is a case in point.

- Benevolence: The expectation that the other party cares about the trustor's interests and needs. Teachers will for example trust their principal when (s) he looks out for them and has their best interest at heart when planning their timetable or managing their performance. Equally, parents will trust teachers who they feel act in the best interest of their child. 
- Integrity: The expectation that the other party will act in a just and fair way, such as when a school principal treats all teachers in the same way, or when a teacher supports all children equally.

Trust underpins the nature of teachers' work, while it also acts as a lubricant for effective collaboration and relations in a school context. We argue that the high level of specialist knowledge and skills required to teach, as well as the independence needed to accommodate various student needs, requires a degree of trust in teachers. Freidson (2001) positions these criteria at the heart of 'professionalism' where professional autonomy to make such discretionary judgements is legitimised by the fact that they place the good of the client, the public or the development of a discipline over their economic self-interest. It is these good intentions that underpin the work of teachers and without which they would fail to be effective. Parents who, for example, do not trust teachers are unlikely to place their child in the care and under the supervision of teachers, while it is also difficult to imagine a student learning from a teacher he/she does not trust. As Gillespie (2015) explains, trust is particularly relevant for relations that have an element of risk, where people have to rely on someone else's skills, knowledge, judgements or actions and where personal information of a sensitive nature needs to be shared as part of someone's role or responsibility and for someone to be able to learn and develop. Risk and reliance on others necessitate trust; without risk there is no need for trust, according to Gillespie (2015).

Trust also allows for learning of, and collaboration between, teachers in a school. Here, teachers are not the object of trust but the actors who need to extend their trust to those in their microsystem to be able to learn and develop. As Tschannen-Moran's (2014) and Cerna's (2014) work indicate, high levels of trust between teachers, or between a teacher and his/her principal leads to more honest and effective forms of collaboration and promotes teachers' effective engagement with professional learning networks and their own professional development. Engagement in turn can positively impact on a schools' innovation potential, it improves teaching practice and student outcomes (see; Bryk and Schneider, 2002; Forsyth et al., 2011; Goddard et al., 2001; Godfrey and Handscomb, 2019). The underlying mechanism is one of taking a risk in sharing professional concerns and becoming vulnerable to judgement of others when for example asking for advice on how to manage a difficult group or teach a difficult subject area. When such trust is reciprocated, teachers are more satisfied and engaged, and experience less burnout.

The reciprocal nature of trust is particularly relevant for trust to grow and deepen over time. Six and Van Ees (2017, p. 61) for example explain how two interacting actors build trust when over time positive expectations are met and both actors gradually get to know one another better. Lewicki and Brinsfield (2015, p. 55) refer to 'feedback loops' in describing how trust changes with evidence in favour of, or against, further trust (trust as an 'evidentiary phenomenon'). Trust relations in a school context are between a teacher and his 
colleagues, students and parents where both sides will have an initial perception of the other one's trustworthiness. Such perceptions will be confirmed over time when each actor acts according to his/her expected role, or rejected when someone proves untrustworthy. A teacher will for example be trusted by parents or students when they teach well and have a student's best interest at heart; while a teacher will trust his/her principal when he/she manages the school well and supports the teacher in his/her work. Trust relationships develop into deeper and more complex levels of attained trust when both sides confirm their competence, benevolence and integrity.

The interactive nature of the trust building process implies that the trustor's predisposing beliefs and expectations, as well as his/her knowledge and skills to assess the counterpart's performance, are crucial for high-trust relations to develop. This applies to all the relations in a school, such as between principals and school boards or parents and principals: each actor in the relationship needs to be competent in the specific role for which he/she has responsibility, as well as have the knowledge and skills (and information) to be able to assess the other person's competence and trustworthiness. Capacity of both trustor and trustee in a school is thus a key part of whether they will come to trust each other and engage in a collaborative process and cycle of learning that will allow teachers to do a good job and develop their work. Where such capacity is lacking on either side of the trust relation, it needs to be build, such as through specific support and training to ensure that both trustor and trustee can effectively fulfil their roles.

Here we turn to our third accountability variable, as accountability interventions are expected to build such capacity and can provide information about someone's trustworthiness. On the microlevel, accountability refers to a specific social relation between an actor and a forum where, in Bovens' (2007, p. 450) and Klijn and Koppenjan's (2014, p. 264) description, the actor has an obligation to explain and to justify his or her conduct, the forum can pose questions and pass judgement, and the actor may face consequences. These consequences can vary from support to sanctions, such as when professional development is offered to teachers who need to improve specific aspects of their work, or who are withheld financial bonuses when test scores of their students are lagging behind.

Teachers can be part of horizontal accountability relations with peers when holding each other accountable for high learning outcomes across the school through peer review, joint practice development or lesson study. These relations are expected to motivate peer-to-peer learning which would in turn benefit student learning and outcomes.

A school typically also has (a set of) vertical accountability relation(s) which are organised around systems of performance management where principals or middle leaders assess and judge teachers. This type of relationship is top-down and in principle one sided where the principal holds the teacher accountable and where teachers are expected to follow command, comply with school standards and learn from the advice that was given to them. 
Both types of accountability relations are often, at least to some extent, informed by the external accountability in the school's exosystem and for example the inspection criteria or testing frameworks used to evaluate school quality. As Ehren et al.'s (2015) work shows, these frameworks are highly influential in setting expectations for schools and their stakeholders and redirect school resources and learning of students and educators towards the standards by which they are judged. By specifying the criteria for judging quality one enters a grey zone between measuring quality and defining it, particularly when the accountability framework includes instructions for schools on how to meet standards, or examples of good practice (see also Smith and Ngoma-Maema, 2003). Such specifications will likely restrict school staff's space in determining what matters to them and tend to motivate or pressurise schools to solely focus on the areas in the school that are measured. As Kelchtermans (2007) explains, the audit procedure used by the Flemish Inspectorate of Education communicated a particular view of a 'good' education, so that schools reacted either with compliance or through an awareness that they needed to justify a different approach. In England, Ofsted scrapped their grading of lessons as placed teachers under pressure to produce intricately detailed lesson plans and to mark student work with a specific frequency type of feedback, scrutinised in particular detail by their head teacher. ${ }^{2}$

The consequences schools face for their performance on the standards shifts the functioning of these standards from measurement to setting expectations about what good teaching and a good school looks like and to communication and informing teachers and schools about key aspects of performance they should focus on.

The examples take us back to our discussion about trust as a condition of effective teaching and learning and development of teachers. How does highstakes accountability, when entering a school context influence trust relations, teachers' willingness to take risks and be vulnerable to learn and develop one's profession? And can trust and accountability be part of the same relationship, such as where a teacher has to trust his/her principal by whom he/she is also evaluated and monitored?

Brennan and Shah (2000) problematise trust-accountability relationships by discussing the effect of assessing teaching quality and how such assessments redefine our understanding of good teaching and potentially reduce teachers' autonomy in defining standards of good teaching. Particularly in a context of high-stakes accountability, 'professionalism' in the pure sense of the word as having discretion over the knowledge and skills that define the profession, setting entry requirements to the occupation and criteria by which the occupation is evaluated (see Freidson, 2001) is greatly reduced. This reduction of autonomy by standardising the quality of the work takes shape over time when new teachers are educated in performative systems and take external accountability standards on the quality of their work for granted. Wilkins (2011), Dougill et al. (2011) and Berry (2012) explain how new young teachers in England will themselves have been educated in a system in which pupils were frequently tested and 
schools were required to meet externally imposed standards and are regularly inspected. According to Berry (2012, p. 402), 'it is all they have known so they don't imagine it can be different'; they have taken on a 'post-performative' identity (Wilkins, 2011). According to Hall and Noyes (2007), teachers who express different views, for example of the importance of individual teacher autonomy, are now considered out of date or incompetent.

We expect that the reduction of autonomy, both on the school level and for individual teachers, potentially reduces and inhibits trust relations in a number of ways. First of all, teachers who teach to a standardised script will expect students to perform to such external standards and have little flexibility or interest in accommodating individual learning needs or interests that fall outside of the accountability framework. Setting standards is inherently at odds with supporting individual differences and interests as is clearly evidenced by practices of 'educational triage' in test-based accountability system. Work by Jennings and Bearak (2014) and Jennings and Sohn (2014) for example show how, in such systems, teachers categorise their students into those who will achieve anyway (and therefore don't require too much input), hopeless cases (who would be a waste of effort) and borderline cases who require attention and input to get their target grades which will improve the school's overall accountability classification. Reduction of teachers' autonomy over their work will have an effect on their trust relations with students, peers or the principal in so far as it reduces their ability to meet individual students' learning needs or to foster their individual talents and interests.

Similarly, teachers will likely struggle to trust their principle when their performance management is motivated by meeting a set of external criteria, particularly when these have little value for their day-to-day work or aspirations for how to develop their teaching and future career. The problem is well exemplified by Schmidt's (2013) case study work in Germany which shows how inspection categories, and judgement of schools along those categories clearly create new meanings of how the profession is viewed, the roles people are expected to fulfil in schools and the types of interventions and knowledge appropriate for those roles, emphasising good practices and strategies to improve high achievement in response to inspections. Schmidt (2013) explains how head teachers, after the introduction of inspections in Germany, were held accountable for the organisational development of their school and had to provide data to the Inspectorate on the development of their school. According to Brauckmann and Pashiardis (2010), the inspection agenda positioned them as an assertive, knowing, responsible and creative entrepreneur, instead of only the bureaucratic head of an institution. School heads tried to fulfil these new requirements by presenting their school to the Inspectorate of Education and also by managing the process of distribution and dissemination of inspection results. Brauckmann and Pashiardis (2010) and O'Brien et al. (2014) explain how head teachers selected and reordered the inspection data according to their own priorities and to the interests of their school, taking on a new role 
in thinking about and providing a framework for the interpretation and use of inspection data by other actors and stakeholders in the school (teachers, parents, students etc.). Höhne (2011) and Tacke (2005) point out how these changes shifted attention of head teachers from pedagogical practices within the classroom (teaching and learning) to an organisational level (evaluating, organising actions on accountability) and emphasised a one-sided focus on managerial organisational knowledge where knowledge about improvement of teaching practices was delegated to external experts. According to Tacke (2005), such an outsourcing of pedagogical and didactical knowledge as a result of an increased focus on organisational issues and development promoted the de-professionalisation of schools and teachers.

External accountability clearly affects interpersonal relations within school and redefines the status of the teaching profession and what is considered high-quality teaching, particularly when informed by an external high-stakes framework. Trust and accountability thus seem ill at odds with one another, particularly when the latter is informed or imposed by an external set of standards over which teachers have little influence or say.

Trust is however an important condition for accountability relations to work. Accountability of teachers' performance, without their active engagement and involvement in providing information on their work, would require continuous observation to understand the quality of their performance. Modern school principals would mitigate these restrictions by using available data on for example student achievement to understand their teachers' performance, but various studies (Datnow et al., 2007; Ingram et al., 2004) emphasise the limitations of such data: student achievement is the result of a complex mix of factors, most of which will be outside of the teacher's span of control, such as a student's home environment and socio-economic background. Various studies also indicate the perverse effects of such strict monitoring, particularly in how it motivates teaching to the test or to inspection indicators and leads to an impoverished curriculum, assessment and learning experience. Effective accountability requires a degree of trust where the teacher feels safe to talk about challenges in his/her work and knowledge or skills he/she lacks where effective performance management focuses on learning and improvement, instead of passing judgement and assigning blame.

Similarly, teachers being reviewed by peers in a horizontal accountability relationship will learn more from the exercise when they trust their peers to ask for advice, instead of having to fear that the assessment will have negative consequences for their employment status or salary. Accountability, both horizontal and vertical, can only improve learning and build capacity when introduced in a high trust, collaborative environment. As O'Neill (2013) and Näslund and Hallström (2017) argue, control and monitoring, in a context of high trust is interpreted as a sign of good intentions and credible concern and will motivate voluntary compliance, but will escalate distrust when introduced in a distrustful relation. 


\section{Mesosystem}

Individual learning of teachers and their in-school relations are embedded in the mesosystem of the school's culture and organisation. How the school is structured in terms of time tabling, opportunities for joint practice development or access to more senior colleagues will allow or restrict staff to learn and develop. The school's structure and culture also takes shape as a result of these microlevel interactions as and when these interactions become structural properties of the school. Trust, accountability and capacity are important properties of a school's organisation which affect relations in the school, while these properties equally emerge from the reciprocal exchanges that take place in the school community.

Moolenaar et al. (2014) for example describes how frequent social exchanges between students, parents and school staff are the foundation of collective trust or a school's social capital. Over time, such interactions become embedded in a system of habitual behaviours, where collective trust and social capital emerge from the ties and social relationships between individuals. Through these ties, a shared understanding of norms and values is created, knowledge is shared and habits are created which influence the behaviours and create hegemonic beliefs for those that work within them.

Shared dispositions and practices, when viewed as a whole, can be referred to as 'culture'; 'an acquired system of habitual behaviour which also generates (and/or determines) individual's schemes of action, opportunities to learn and an understanding and practice of 'this is how we do things around here'. These understandings and practices (or social structures) in turn reinforce an existing culture when new teachers are for example being socialised into the culture of the school. The reinforcing effect makes a school's culture or social structure and capital often hard to change. A school in which teacher-administrator friction seems endemic may for example find that a new principal can create a few relationships with key teachers who only gradually come to trust a new principal when having had a long institutionalised expectation of conflict (Macmillan and Meyer, 2004).

A school's social capital and culture of (collective) trust is an important element of a school's capacity to improve and perform well. Forsyth et al. (2011) find a positive relation between collective trust in a school and student outcomes. The underlying mechanism is one of sharing information, shared decision-making, collaborative planning of for example curriculum, assessment and time tabling, and consistency in behavioural policy which are all conducive to student learning. These school-level variables are well evidenced in various school effectiveness studies, such as by Scheerens (2014), Reynolds et al. (2014), Muijs et al. (2014) and Hopkins et al. (2014) who found the following school-level conditions to generate high learning outcomes:

- Achievement orientation and high expectations: a productive school climate, a school mission focused on achievement, shared vision and goals, high expectations that all students can achieve 
- Cooperative atmosphere and an orderly climate: cooperative planning, a learningoriented atmosphere consensus, orderly climate

- Clear goals on basic skills: focus on student learning, concentration on teaching

- Frequent evaluation: appropriate monitoring, evaluative potential of the school, assessment

- Professional development: staff development, in-service training, a learning organisation

- Parental involvement: parent support, home school partnership

- Strong leadership: educational leadership, school management and organisation, improvement-oriented leadership

- Effective instructional arrangements: classroom management, time on task, structured teaching, opportunity to learn, coordination in curriculum and instruction

School-based systems of evaluation and monitoring can support the development of such cultures and practices when they create a shared understanding of what standards of good teaching look like in the school, and generate information for various actors to bring their work in line with standards of school effectiveness. Systems for school self-evaluation and quality assurance aim to have this effect by engaging the school community in a continuous cycle of 'plan', 'do', 'check', 'act' and providing information to teachers and head teachers on where and how to improve educational processes in the school and learning outcomes of students.

Nelson et al.'s (2015) review of effects of school internal evaluation however shows that this is a notoriously difficult process which is often unstructured, lacks follow-up and is ill-informed by unreliable or inaccurate data. In order for internal evaluation to be successful in objectively examining the school context and the learning of students and in using the findings to prompt discussion about improvement, a number of conditions must be in place. School-internal accountability and evaluation needs capacity of school staff to collect, interpret and act on high-quality data, a skill that has been termed 'evaluation' or 'data literacy'. Schools also need time for developing and embedding evaluation literacy and collaborative discussion, while access to valid and reliable data can support the assessment and improvement of the quality of teaching in the school. The importance of effective leadership is a recurring theme in the literature on internal evaluation where leadership activity may be directly in support of internal evaluation, for example through modelling data use or in leading collaborative discussions, or indirect in ensuring that resources are provided and that a culture is developed which is improvement oriented and enables critical reflection and challenge to existing practices. As Earley and Bubb (2014) comment, leadership needs to create and embed an inquiry-oriented culture and needs to provide the resources, particularly of time, to allow staff to collaborate, agree common goals for inquiry and discuss findings and to put support in place for staff to develop research expertise. 
These findings highlight how trust also acts as a precursor to effective school-internal accountability and evaluation. A climate of trust in the school, underpinned by supportive relationships and good communication, allows for collaborative working in which teachers can be critical in their analysis of data and challenge one another openly, according to Nelson et al. (2015). Timperley (2008) found that in the most improved school of the three she studied, teachers were more ready to express uncertainty in group discussions and to seek help from others in the group when there was a collaborative (high trust) climate in the school. Such a climate enables distributed leadership and shared ownership to develop in internal evaluation (Montecinos et al., 2014). The importance of trust, the absence of blame and a history of working together is also emphasised by Earley and Bubb (2014); where trust and a history of working together was established, with values and expectations that supported open, critical inquiry around data and instruction, data use to improve instruction improved more rapidly.

A culture of trust in the school not only refers to a climate and culture of supportive relations between school staff, but also encompasses an element of moral values and institutional ethics which would strengthen benevolent and fair behaviour of individual teachers. Freidson (2001, p. 16) describes institutional ethics as 'the moral legitimacy of the policies and institutions that constrain the possibility to practice in a way that benefits others and services the transcendent value of discipline'. Institutional ethics need to support a moral concern for the ultimate purpose of a discipline which would, in our case, be the learning and well-being of students. The recognition and monitoring of any violation of ethical and moral values, such as when teachers use school resources for their personal gain, is needed to ensure that students and parents can trust teachers, as well as to ensure the school has the capacity to improve. As Meier (2004) explains, corruption diverts money and supplies away from schooling and reduces opportunities to improve learning, such as when teachers sell places in the front rows of their overcrowded classrooms, when parents have to pay if they wanted their child's exercise books to be corrected, or when the only way a child can complete the curriculum in an examination year is through private tuition with the same teacher.

In the mesosystem, capacity thus refers to school-level conditions conducive to student outcomes, and which create an environment for teachers to teach well and improve their performance. Trust is an important element of such an environment and conceptualised here as a school's social capital, collective trust, supportive culture and ethical climate. School-level structures of evaluation and monitoring can promote such conditions by creating a shared understanding of high-quality teaching and providing feedback for improvement. Trust is also an important precursor for such evaluation and monitoring to work well as performance management or peer-to-peer accountability will likely be meaningless when teachers are unwilling to risk openness about their weaknesses and concerns and learn about how to improve them, or silently or actively oppose an evaluation of their work. 


\section{Exosystem}

Learning of school staff and their relations in schools are embedded in a wider institutional context and structures, such as the regulation of teacher quality, safeguarding in schools or standards on curriculum and assessment. We are interested in how trust, capacity and accountability can be positioned as elements of the exosystem and as such affect interpersonal relations in the school and opportunities for learning and development, both directly, as through the mesosystem of the school.

When defined on the level of the exosystem, trust is no longer only about an individual school and whether the school staff can be trusted or whether the school has an internal culture of high trust. Here our definition of trust expands to encompass the education system as a whole, and the safeguards and institutional arrangements in place to ensure the quality of schools and other stakeholders in the system involved in the education of pupils. This wider institutional trust or system trust is 'the expectation of appropriate behaviour in organised settings based on the established norms of that institution' (see Bachmann, 2001). Parents who trust that teachers in general will do their utmost to try and educate and protect their child during school hours will base that expectation on experiences of other parents or how the media reports about teachers and schools. Trust in education systems is not a given and varies across countries. Cerna (2014) shows that countries vary on the trust in education where confidence levels in some countries have gone down substantially (e.g. Brazil, Chile), whereas in other countries, these levels have increased (Finland, Israel, the Netherlands).

In the context of political science, Beugelsdijk (2005) and Rothstein (2013) refer to macrosources of trust, confidence or generalised trust that arise from an institutional environment of laws, norms and standards. Individuals are more likely to engage in a relationship and trust someone else when they feel protected by a well-functioning bureaucracy (legal, political and economic). A well-functioning bureaucracy ensures that people or organisations who cannot be trusted are sanctioned, setting a structure and culture in which individuals are able to act in a trustworthy manner and without risk, and in which they can reasonably expect that most others will generally do the same (Rothstein, 2013). Parents who trust schools in general would for example expect there to be institutional safeguards which protect their children in school and which ensure a good quality of education.

Accountability systems are premised on providing such safeguards and are an important part of the bureaucracy in which schools and school staff operate and how the public comes to trust teachers and schools. Accountability systems set standards of good teaching and/or student outcomes, ensure compliance to regulations on for example safeguarding or qualifications of teachers in schools and, as such, inform the work of school staff, and also the public's and parents' trust in schools. In a well-functioning accountability system, parents will expect teachers in general to be knowledgeable and fair and schools to be safe places to send their child to. A well-functioning accountability system also ensures schools' integrity 
in using resources for the primary purpose of their work. As we explained earlier, teachers or schools who use resources for their personal gain and place personal gain above the obligation to do good work for their students, lose their legitimacy in the eyes of the public and students they serve. According to Freidson (2001), professionals only have legitimacy in so far as they act ethnically, where strong institutional arrangements can ensure appropriate and ethical behaviour.

Here, the institutional structures of resourcing schools, training and professional development of school staff and opportunities for capacity-building are also important. The conventional focus in defining capacity is on the acquisition of financial and physical resources but simply having those resources does not mean they will be used well or appropriately. Capacity, according to Hatch (2013), also depends on whether, and how a school turns those resources into outcomes and, on the exo-level, this requires policies, structures or more generally 'the ability of people, organisations and society as a whole to manage their affairs successfully' (OECD, 2017, p. 7).

The relevancy of such organisational capacity on the system-level is well exemplified in recent work by Levy et al. (2018) who present various examples of a weak bureaucracy in South Africa and how divergent and competing regional interests, organisational cultures, patronage and repeated leadership turnover in the Eastern Cape contributed to chronic weaknesses in both financial and personal management of the education system in the province. The hierarchical weakness and lack of coordinating capacity caused a resistance to change where the system remained stuck in low-quality education and outcomes of students. Capacity to produce high learning outcomes thus requires technical capital (money, physical materials and resources), human capital (the skills, knowledge and dispositions of the personnel involved), as well as social capital (the networks, norms of trust and collaboration and collective commitment among those involved).

The accountability system again has a function in building such system-level capacity, such as when the information from inspections or high-stakes testing is used to redistribute resources to those that need it most. De Grauwe (2007) refers to a 'liaison function' of Inspectorates of Education who act as an agent between the top of the education system (where norms and rules are set) and the schools (where education is shaped and takes place). They liaise between the two by informing schools of decisions taken by the centre or by informing the centre of the realities at school level. The annual and thematic reports, published by many Inspectorates of Education, are a clear example of the liaison role of inspections. These reports, which provide a summary of aggregated school inspection assessments and sometimes of specific policy relevant themes, allow national governments to monitor the performance of the education system and adjust their policy to improve system performance.

The liaison role of Inspectorates of Education can also include establishing good linkages with other services involved in quality development of schools such as pre- and in-service teacher training, curriculum development, preparation of national tests and examination and identifying and spreading new ideas 
and good practices between schools. This role becomes increasingly important when school systems are decentralised and accountability shifts from vertical topdown approaches to more decentralised structures or horizontal accountability. In horizontal systems, Inspectorates of Education can have a role in identifying and spreading new ideas and good practices between schools, particularly when ambitious reform programmes are being launched. Liaison can also include establishing good linkages with other services involved in quality development such as preand in-service teacher training, curriculum development, preparation of national tests and examination and allowing emerging lessons about effective pedagogy to disseminate through the system. Levy et al. (2018) refer to a 'transmission belt' as an element of effective governance where reform is effectively implemented through a gradual, negotiated approach instead of confrontation. Where coalitions of system actors work towards shared goals, reforms are more likely to succeed, according to a World Development Report (2018, in Levy, 2018).

\section{Macrosystem}

The macrosystem encompasses any group (culture, subculture or other extended social structure) whose members share value or belief systems, resources, hazards, lifestyles, opportunity structures, life course options and patterns of social interchange (Tudge et al., 2009; Bronfenbrenner, 1989, p. 25). The macrosystem envelops all other systems but will only have an effect on a developing person if it influences the microsystem in which the person is situated, according to Tudge (2009). Value systems vary substantially across countries, particularly in how groups conceptualise 'good teaching' or how they would understand the purpose of education, and the best strategies to coordinate or reform education. Such values will affect how capacity is build, resources are allocated and how accountability is organised and trust develops between people.

A study by Mourshed et al. (2010) shows some of the major differences between countries. Education leaders in Anglo-American systems (the United States, England, Canada) in their study talked about the importance of school autonomy, parental choice and external accountability, giving prominence to quantitative targets on student outcomes and sharing these with a broader public. In contrast, leaders in Asian and Eastern European systems, according to Mourshed et al. (2010), argued against setting quantitative targets, preferring to share performance data with individual schools, engaging them in a private dialogue about how they can improve. In this latter group, only system-level data is generally made available publicly. One of the education leaders from Singapore explained in their study (p. 68/69) that

Singapore's value system is that first priority is the nation, second is the organization, and third is the individual. We do not need to use targets because the greater good of the nation motivates our people to always work towards improvement. 
According to this respondent, in systems that set targets for schools and individuals, these targets are needed to create common ground for improvement.

In Eastern Europe, such targets were also considered inappropriate given the historic context of strong centralisation. One of the respondents in Mourshed's et al. (2010, p. 69) study said that 'our schools and educators want freedom to do what they think is best. Performance targets would be viewed as excessive state control and would be rejected'.

These different values reflect a broader ideology and national culture and preference for a 'default' governance approach. Meuleman (2015) explains that Anglo-Saxon countries generally have a tradition of liberal economics, where Northwestern Europe is more network-driven and central, and eastern and southern Europe and many Asian and South American countries are more hierarchical and central. Following this logic, we would expect these countries to either introduce market mechanisms to coordinate improvement, standardise schooling through top-down hierarchical control, or emphasise collaboration in local school improvement networks and partnerships.

Each of these three ideal types represents a different view on the purpose of education. As Greany and Earley (2017) and Greany and Higham (2018) describe, in markets, the core belief is that customer orientation and competition create efficiency and innovation when self-interested parents and educators ensure flexibility and high quality in offer of services. A market-based system, according to Greany and Earley (2017), reflects a change in thinking away from seeing education as primarily a public good, the dominant logic of hierarchies, to seeing it as primarily a private one. Rather than valuing education because it contributes to a robust democracy, offers the chance of personal fulfilment or the advancement of knowledge, a market-based logic is premised on the ideas that we must invest in our human capital because doing so will improve our personal and national competitiveness in a global economy (Greany and Earley, 2017).

The introduction of market mechanisms in education with subsequent changes to roles and responsibilities of teachers and principals has clear implications for trust in education systems, as well as interpersonal trust between teachers, students and parents. Glatter (2017) cites Marquand (2014, p. 103) who says 'citizens will trust their leaders if they think public institutions are governed by an ethic of equity and service, but if the public realm succumbs to invasion by the market or private realms, citizenship becomes an empty shell and public trust withers'. Where there is no trust in the public education system, or even distrust, the historical relationship or social contract between schools and society is eroded and teachers and schools are increasingly accused of education decline. This is exemplified within the English education system where the introduction of new public management approaches in the 1970s positioned the parent as consumer and changed the high trust, optimistic and collegial culture of the Post War consensus into distrust of the performance of public services in general and more specifically in teachers. 
In a hierarchical system, where education is centrally organised by the state, the dominant logic is to see education primarily as a public good, rather than a private one, according to Greany and Earley (2017). The quest for equal access and opportunities would lead central governments to regulate school quality through top-down processes of decision-making. Societies would value the public realm of service, equity, professional and public duty, and this would inform centralised political decision-making and education reform (see Glatter, 2017). Governments are seen as the key player in ensuring high-quality education and would prescribe the processes through which teaching is delivered. The underlying assumption here is, according to Meuleman (2008) and Freidson (2001), one of standardisation and routinisation, such as through the implementation of national curricula or a set of standardised assessments in order to prevent and replace arbitrary authoritarianism and nepotism.

Our final ideology or value-system is one of 'co-production' where good education would be conceptualised as a mutual effort of teachers, students, parents and other care givers, instead of an individual teacher. Coproduction is described by Brandsen and Honingh (2016) as 'a relationship between a paid employee of an organization and (groups of) individual citizens that requires a direct and active contribution from these citizens to the work of the organization'. In education settings, those relations would for example involve parents where research (Honingh et al., 2018; Fledderus et al., 2014) shows a positive effect of active parental participation in children's schooling. More recent reforms, such as in England's promotion of school-led improvement (Greany and Higham, 2018) or Northern Ireland's area-learning communities extend these relations to include collaboration with other schools, teachers, school leaders and service providers. High-quality education here is conceptualised as an outcome of collective action of a multitude of stakeholders, and trust is the underpinning principle that would allow for such collaboration. Examples are of primary and secondary schools working together in developing a crossphase mathematics curriculum and thereby improving student outcomes across students' compulsory school career.

Chapter 2 describes these ideal types of education systems in more details and how trust, accountability and capacity-building are situated within the various forms of governance.

\section{Chronosystem}

Bronfenbrenner's model situates time in each of the other subsystems, reminding us of the need to examine the relative developmental pace of change from the perspective of actors at different levels in the ecosystem. In the microsystem, time refers to 'engines of development', such as specific activities or encounters and interactions which change over time when a child grows older, his/her environment changes and interactions with the environment change (Tudge et al., 2009). In our use of the model, we are interested in the types of activities and 
interactions teachers engage in and learn from, and how these change over time when they become more experienced or their school environment changes.

On the meso-level, time denotes organisational development and learning where we can think of how culture and structure change over time as a function of learning of individuals and external impulses. Changes and patterns of improvement in the exo and macrosystem are typically referred to as 'macro time'. Relevant questions here are how attempts to improve teaching practice are set within the context of often rapid policy changes. These patterns are not well understood as the effects of competition in a market system for example work through the involvement of many actors in the process, such as districts, schools principals and teachers in classrooms. It is not clear from the outset whether responses by some actors, for example, principals, may be more important than others, for example. Neither is it clear how behavioural changes across different actors work through the various subsystems. As Godfrey and Handscomb (2019) point out, it can take teachers months or years to implement a new curriculum due to the need to build new skills, introduce new materials and refine strategies to context, while governments can impose policy changes that have dramatic implications for the school curriculum in the space of a few weeks.

Given the high dependence of responses of various actors on the context in which they operate, including the distribution of autonomy between various actors in the system, the specific mix of policy interventions and the historic context in which these are set, responses of educators over time will be impossible to predict and relate to specific interventions. Lemke and Sabelli (2008) refer to education as a complex system where every causal chain is mediated, and many chains branch and loop back on themselves in complicated webs of mutual interdependent, self-regulation, and amplification of effects. Relevant questions to consider are how initial levels of capacity, trust/distrust and accountability or variations of these three promote or inhibit change. How do educators for example respond to the introduction of high-stakes accountability in a context of distrust and lack of capacity to meet accountability targets? Or how does trust and capacity develop in a context without external accountability and information about the behaviour and performance of educators? Can we think of situations where high trust and high-stakes accountability either improve or reduce capacity to ensure high learning outcomes? And how can we understand these questions when education systems transition between various types of governance?

We'll revisit these questions in our concluding chapter, reflecting on the country chapters in part two of this book to present some of the main storylines of how trust, accountability and capacity change over time.

\section{Notes}

1 https://www.researchgate.net/publication/321012999_Bronfenbrenner\%27s_ Bioecological_Theory_Revision.

2 https://www.teachers.org.uk/edufacts/appraisal. 


\section{References}

Addison, S. J. (2015). Using scenarios as part of a concurrent mixed methods design (pp. 154-170). In: F. Lyon, G. Mőllering, \& M. Saunders (Eds.), Handbook of Research methods on Trust. Cheltenham/Northampton: Edward Elgar Publishing.

Bachmann, R. (2001).Trust, power and control in trans-organizational relations. Organization Studies, 22(2), 337-365.

Bachmann, R., \& Zaheer, A. (Eds.) (2006). Handbook of Trust Research. Cheltenham: Edward Elgar Publishing.

Barber, M., \& Mourshed, M. (2007). How the World's Best-Performing Schools Systems Come Out On Top. London/Washington: McKinsey \& Company.

Bew, P. (2011). Independent Review of Key Stage 2 Testing, Assessment and Accountability. TSO.

Basma, B., \& Savage, R. (2018). Teacher Professional Development and Student Literacy Growth: a Systematic Review and Meta-analysis. Educational Psychology, 30, 457. https://doi.org/10.1007/s10648-017-9416-4.

Berry, J. (2012). Teachers' professional autonomy in England: Are neo-liberal approaches incontestable? FORUM, 54(3), 397-409.

Beugelsdijk, S. (2005). A note on the theory and measurement of trust in explaining differences in economic growth. Cambridge Journal of Economics, 30(3), 371-387.

Brandsen, T., \& Honingh, M. (2016). Distinguishing different types of coproduction: A conceptual analysis based on the classical definitions. Public Administration Review, 76(3), 427-435.

Brauckmann, S., \& Pashiardis, P. (2010). The clash of the evaluations: In search of the missing link between school accountability and school improvement-experiences from Cyprus. International Journal of Educational Management, 24(4), 330-351.

Bray, M., Adamson, B., \& Mason M. (2014). Different models, different emphases, different insights. In: M. Bray, B. Adamson, \& M. Mason (Eds.), Comparative Education Research. CERC Studies in Comparative Education, vol 19. Cham: Springer.

Bray, M., \& Jiang, K. (2014). Chapter 5. Comparing systems. In: M. Bray et al. (Eds.), Comparative Education Research: Approaches and Methods, CERC Studies in Comparative Education, vol 10. Switzerland: Springer International Publishing.

Bronfenbrenner, U. 1989. Ecological systems theory. Annals of Child Development. 6, 187-249.

Bovens, M. (2007). Analysing and assessing accountability: A conceptual framework 1. European Law Journal, 13(4), 447-468.

Burns, T. (2012), Getting it right: Capacity building for local stakeholders in education. Governing Complex Education Systems Background Document.EDU/CERI/CD/RD(2012)2.

Burns, T., \& Cerna, L. (2016). Enhancing Effective Education Governance. Paris: OECD publishing.

Brennan, J., \& Shah, T. (2000). Quality assessment and institutional change: Experiences from 14 countries. Higher Education, 40(3), 331-349.

Bryk, A., \& Schneider, B. (2002). Trust in Schools: A Core Resource for Improvement. New York: Russell Sage Foundation.

Cerna, L. (2014). Trust: What it is and why it matters for governance and education. OECD Education Working Papers, No. 108. Paris: OECD Publishing. http://dx.doi. org/10.1787/5jxswcg0t6wl-en.

Coffield, F. (2012). Why the McKinsey reports will not improve school systems. Journal of Education Policy, 27(1), 131-149.

Datnow, A., Park, V., \& Wohlstetter, P. (2007). Achieving with data. In: How High Performing School Systems Use Data to Improve Instruction for Elementary Students. San Francisco: Center on Educational Governance University of California. 
De Grauwe,A. (2007). Module 1; Supervision, A Key Component in a Quality Monitoring System. http://www.iiep.unesco.org/fileadmin/user_upload/Cap_Dev_Training/Training_ Materials/Supervision/SUP_Mod1.pdf.

Dougill, P., Raleigh, M., Blatchford, R., Fryer, L., Robinson, C., \& Richmond, J. (2011). To The Next Level: Good Schools Becoming Outstanding. Reading: CfBT Education Trust.

Earley, P., \& Bubb, S. (2014). Data and inquiry driving school improvement: Recent developments in England. Journal of Educational, Cultural and Psychological Studies (ECPS Journal), 1(9), 167-184.

Ehren, M. C., Gustafsson, J. E., Altrichter, H., Skedsmo, G., Kemethofer, D., \& Huber, S. G. (2015). Comparing effects and side effects of different school inspection systems across Europe. Comparative Education, 51(3), 375-400.

Ehren, M. C. (Eds.) (2016). Methods and Modalities of Effective School Inspections. Dordrecht: Springer International Publishing.

Freidson, E. (2001). Professionalism, the Third Logic: On the Practice of Knowledge. University of Chicago press.

Fledderus, J., Brandsen, T., \& Honingh, M. (2014). Restoring trust through the co-production of public services: A theoretical elaboration. Public Management Review, 16(3), 424-443.

Forsyth, P. B., Adams, C. M., \& Hoy, W. K. (2011). Collective Trust: Why Schools Can't Improve without It. New York: Teachers College Press.

Gillespie, N. (2015). Survey measures of trust in organizational contexts: An overview (pp. 225-240). In: F. Lyon, G. Mőllering, \& M. Saunders (Eds.) Handbook of Research methods on Trust. Cheltenham/Northampton: Edward Elgar Publishing.

Glatter, R. (2017). 'Because we can': Pluralism and structural reform in education. London Review of Education, 15(1), 115-125.

Gundlach, G.T., \& Cannon, J. P. (2010). "Trust but verify”? The performance implications of verification strategies in trusting relationships. Journal of the Academy of Marketing Science, 38(4), 399-417.

Goddard, R., Tschannen-Moran, M., \& Hoy W. (2001). A multilevel examination of the distribution and effects of teacher trust in students and parents in urban elementary schools. Elementary School Journal 102(1): 3-17.

Godfrey, D. (2014). Creating a research culture - Lessons from other schools. SecEd.

Godfrey, D., \& Handscomb, G. (2019). Evidence use, research-engaged schools and the concept of an ecosystem (p. 4). In: An Ecosystem for Research-Engaged Schools: Reforming Education Through Research. London: routledge.

Greany, T., \& Higham, R. (2018). Hierarchy, Markets and Networks:Analysing the 'Self-Improving School-Led System' Agenda in England and the Implications for Schools. London: IOE press.

Greany, T., \& Earley, P. (2017). Introduction: School Leadership and Education System Reform. London: Bloomsbury.

Hall, C., \& Noyes, A. (2007). The impact of school self evaluation processes on British teachers' views of their work and professionalism. In: Paper presented at the BERA, London. Available from http://www.leeds.ac.uk/educol/documents/167834.doc.

Hatch, T. (2013). Beneath the surface of accountability: Answerability, responsibility and capacity-building in recent education reforms in Norway. Journal of Educational Change, $14(2): 113-138$.

Härkönen, U. (2005). The Bronfenbrenner ecological systems theory of human development. Children, 44, 45.

Höhne, T. (2011). Pädagogische Qualitologie: Zur Transformation von Bildungsforschung in Qualitätsforschung und deren (möglichen) Effekten auf erziehungswissenschaftliches Wissen (pp.139-164). In: R. Reichenbach, N. Ricken, \& H.-C. Koller (Eds.), 
Schriftenreihe der Kommission Bildungs- und Erziehungsphilosophie in der Deutschen Gesellschaft für Erziehungswissenschaft. Erkenntnispolitik und die Konstruktion pädagogischer Wirklichkeiten. Paderborn, München, Wien, Zürich: Schöningh.

Honingh, M., Bondarouk, E., \& Brandsen, T. (2018). Co-production in primary schools: A systematic literature review. International Review of Administrative Sciences. 86(2), 222-239. https://doi.org/10.1177/0020852318769143.

Hopkins, D., Stringfield, S., Harries, A., Stoll, L., \& Mackay, T. (2014). School and system improvement. School Effectiveness and School Improvement, 25, 257-281.

Ingram, D., Seashore Louis, K., \& Shroeder, R. G. (2004). Accountability policies and teacher decision making: Barriers to the use of data to improve practice. Teachers College Record, 106(6), 1258-1287.

Jennings, J. L., \& Bearak, J. M. (2014). "Teaching to the test" in the NCLB era: How test predictability affects our understanding of student performance. Educational Researcher, 43(8), 381-389.

Jennings, J., \& Sohn, H. (2014). Measure for measure: How proficiency-based accountability systems affect inequality in academic achievement. Sociology of Education, 87(2), 125-141.

Johnson, Eileen S. (2008) "Ecological Systems, Complexity, and Student Achievement: Towards an Alternative Model of Accountability in Education," School Leadership Re view: Vol. 3: Iss. 3, Article 4. Available at: https://scholarworks.sfasu.edu/slr/vol3/iss3/4.

Kelchtermans, G. (2007). Macropolitics caught up in micropolitics: The case of the policy on quality control in Flanders (Belgium). Journal of Education Policy, 22(4), 471-491.

Klijn, E. J., \& Koppejan, J. F. M. (2014). Accountable networks (pp. 242-258). In: M. Bovens, R. Mark, E. Goodin, \& T. Schillemans (Eds.), The Oxford Handbook of Public Accountability. Oxford: OUP.

Le Gall,V., \& Langley, A. (2015). An abductive approach to investigating trust development in strategic alliances (pp. 36-46). In: F. Lyon, G. Mőllering, \& M. Saunders (Eds.), Handbook of Research methods on Trust. Cheltenham/Northampton: Edward Elgar Publishing.

Lemke, J. L., \& Sabelli, N. H. (2008). Complex systems and educational change: Towards a new research agenda. Educational Philosophy and Theory, 40(1), 118-129.

Levy, B., Cameron, R., Hoadley, U., \& Naidoo, V. (2018). The Politics and Governance of Basic Education: A Tale of Two South African Provinces. Oxford: Oxford University Press.

Lewicki, R. J., \& Brinsfield, C. (2015). Trust research: Measuring trust beliefs and behaviours (pp. 46-65). In: F. Lyon, G. Mőllering, \& M. Saunders (Eds.), Handbook of Research Methods on Trust. Cheltenham/Northampton: Edward Elgar Publishing.

Lyon, F. (2006). Managing co-operation: Trust and power in Ghanaian associations. Organization Studies, 27(1), 31-52.

Lyon, F., Mőllering, G., and Saunders, M.N.K. (2015). Introduction. Researching trust: the ongoing challenge of matching objectives and methods, 1-25. In: F. Lyon, G. Mőllering, and M. Saunders (Eds). Handbook of Research methods on Trust. Cheltenham/ Northampton: Edward Elgar Publishing.

Mansell, W., \& James M., Assessment Reform Group (2009). Assessment in Schools. Fit for Purpose? London: Economic and Social Research Council. A Commentary by the Teaching and Learning Research Programme.

Macmillan, R. B., \& Meyer, M. (2004). Trust and its role in principal succession: A preliminary examination of a hierarchy of trust. Leadership and Policy in Schools, 3(4), 275-294.

Meadows, D. H. (2008). Thinking in systems: A primer. chelsea green publishing.

Meier, B. (2004). Corruption in the Education Sector: An Introduction. Report for Transparency International. 
Meuleman, L. (2008). Public Management and the Metagovernance of Hierarchies, Networks and Markets: The Feasibility of Designing and Managing Governance Style Combinations. Dordrecht: Springer Science \& Business Media.

Meuleman, L. (2015). Owl meets beehive: How impact assessment and governance relate. Impact Assessment and Project Appraisal, 33(1), 4-15.

Muijs, D., Creemers, B., Kyriakides, L., Van der Werf, G., Timperley, H., \& Earl, L. (2014). Teaching effectiveness. A state of the art review. School Effectiveness and School Improvement, 24, 231-256.

Montecinos, C., Madrid, R., Fernández, M., \& Ahumada, L. (2014). A goal orientation analysis of teachers' motivations to participate in the school self-assessment processes of a quality assurance system in Chile. Educational Assessment, Evaluation \& Accountability, 26(3), 241-261.

Moolenaar, N., Karsten, S., Sleegers, P., and A. Daly (2014). "Linking social networks and trust at multiple levels: Examining Dutch elementary schools", in D.Van Maele, P. Forsyth and M.Van Houtte (eds), Trust and school life: The role of trust for learning, teaching, leading, and bridging. Dordrecht: Springer, pp. 207-228.

Mourshed, M., Chijioke, C., \& Barber, M. (2010). How the World's Most Improved School Systems Keep Getting Better. London: McKinsey. Verfügabr unter: https://www.mckinsey.com/ industries/public-and-social-sector/our-insights/how-the-worlds-best-performingschool-systems-come-out-on-top[Zugriffam 17.03.2015].

Näslund, L., \& Hallström, K. T. (2017). Being everybody's accomplice: Trust and control in eco-labelling (p. 145). In: Trust in Regulatory Regimes. Cheltenham: Edward Elgar Publishing.

Nelson, R., Ehren, M., \& Godfrey, D. (2015). Literature Review on Internal Evaluation. London: Institute of Education.

O’Brien, S., McNamara, G., \& O’Hara, J. (2014). Critical facilitators: External supports for self-evaluation and improvement in schools. Studies in educational evaluation, 43, 169-177.

OECD (2017). Key Concepts and Messages Identified in GCES Work; Working Document A Prepared for Strategic Education Governance Advisory Group Meeting 18-19 September 2017. Paris: OECD.

Office for Standards in Education, Children's Services and Skills. (2012b). Good practice resource - Engaging able mathematics students: King Edward VI Camp Hill School for Boys, Manchester: Ofsted.

O’Neill, O. (2013). Intelligent accountability in education. Oxford Review of Education, 39(1), $4-16$.

Oomsels, P., \& Bouckaert, G. (2017). Interorganisational trust in Flemish public administration: Comparing trusted and distrusted interactions between public regulatees and public regulators (p. 80). In: Trust in Regulatory Regimes. Cheltenham: Edward Elgar Publishing.

Perryman, J. (2006). Panoptic performativity and school inspection regimes: Disciplinary mechanisms and life under special measures. Journal of Education Policy, 21(2), 147-161.

Puranam, P., \& Vanneste, B. S. (2009). Trust and governance: Untangling a tangled web. Academy of Management Review, 34(1), 11-31.

Reynolds, D., Sammons, P., De Fraine, B., Townsend, T., Van Damme, J., Teddlie, C. \& Stringfield, S. (2014). Educational Effectiveness Research (EER): A state of the Art Review. School Effectiveness and School Improvement, 25(2), 197-230.

Rothstein, B. (2013). Corruption and social trust: Why the fish rots from the head down. Social Research, 80(4), 1009-1032.

Six, F., \& Verhoest, K. (Eds.) (2017). Trust in Regulatory Regimes. Cheltenham: Edward Elgar. 
Schmidt, M. (2013). Schulleitungen im Prozess der externen Evaluation (pp. 27-45). In B. Drinck, D. Flagmeyer, D. Diegmann, M. Schmidt, J. Keitel, R. Schubert, \& K. Herzog (Eds.), RuN-Studie. Rezeption und Nutzung von Ergebnissen der externen Evaluation an sächsischen Grundschulen, Mittelschulen und Gymnasien. Radebeul: Sächsisches Bildungsinstitut.

Six, F. E., \& Van Ees, H. (2017). When the going gets tough: Processes of trust building and repair in regulatory relations (pp. 60-79). In: Six, F. E. en Verhoest, K. (Eds.), Trust in Regulatory Regimes. Cheltenham: Edward Elgar.

Seashore Louis, K. S. (2007). Trust and improvement in schools. Journal of educational change, $8(1), 1-24$.

Scheerens, J. (2009). Review and Meta-analyses of School and Teaching Effectiveness. The Netherlands: Department of Educational Organization and Management. www.iqb. hu-berlin.de/lehre/dateien/rapportScherens.pdf.

Scheerens, J. (2014) School, teaching and system effectiveness: some comments on three state of the art reviews. School effectiveness and School Improvement, 25, 282-290.

Smith, W. J., \& Ngoma-Maema, W.Y. (2003). Education for all in South Africa: Developing a national system for quality assurance. Comparative Education, 39(3), 345-365.

Snyder, S. (2013). The simple, the complicated, and the complex: Educational reform through the lens of complexity theory. Paris: OECD Education Working Papers, No. 96, OECD Publishing. http://dx.doi.org/10.1787/5k3txnpt1lnr-en.

Tacke,V. (2005). Schulreform als aktive Deprofessionalisierung?: Zur Semantik der Lernenden Organisation im Kontext der Erziehung (pp. 165-198). In:T. Klatetzki, \& V.Tacke (Eds.), Organisation und Gesellschaft. Organisation und Profession, 1st ed. Wiesbaden:VS Verlag für Sozialwissenschaften.

Timperley, H. (2008). Teacher professional learning and development. Educational Practices Series-18. UNESCO International Bureau of Education.

Tschannen-Moran, M. (2014). The interconnectivity of trust in schools (pp. 57-81). In: D.Van Maele, P. Forsyth, \& M.Van Houtte (Eds.), Trust and School Life:The Role of Trust for Learning, Teaching, Leading and Bridging. Dordrecht: Springer.

Tudge, J. R. H. et al. (2009). Uses and misuses of Bronfenbrenner's bioecological theory of human development. Journal of Family Theory \& Review, 1(4), 198-210.

Thurlings, M., \& den Brok, P. (2017). Learning outcomes of teacher professional development activities: A meta-study, Educational Review, 69:5, 554-576, doi: 10.1080/00131911.2017.1281226.

Vélez-Agosto, N. M., Soto-Crespo, J. G., Vizcarrondo-Oppenheimer, M.,Vega-Molina, S., \& García Coll, C. (2017). Bronfenbrenner's bioecological theory revision: Moving culture from the macro into the micro. Perspectives on Psychological Science, 12(5), 900-910.

Wilkins, C. (2011). Professionalism and the post-performative teacher: New teachers reflect on autonomy and accountability in the English school system. Professional Development in Education, 37(3), 389-409. 


\title{
2 \\ GOVERNANCE OF EDUCATION SYSTEMS
}

\author{
Trust, accountability and capacity \\ in hierarchies, markets and networks
}

\author{
Melanie Ehren and Jacqueline Baxter
}

\section{Introduction}

Chapter 1 briefly touched on the institutional structure of education systems and how systems vary in values and beliefs about opportune reform strategies. We explained how hierarchical systems are organized around a set of top-down relations of dependency and value education as a public good and for its role in contributing to a robust democracy. Markets, we argued, are premised on a different set of values where education is seen as a commodity to prepare individuals for the economic arena, and education systems are coordinated through a set of independent buyer-supplier relations. Our final archetype of network governance conceptualizes education as the outcome of collection action between for example teachers and parents, or even between schools and other service providers. A set of interdependent, high-trust, relations allow these actors to coordinate their work. The various value systems and ways of governing education systems each position actors into different roles and with different responsibilities. This chapter explores the three archetypes in more detail and how trust, accountability and capacity feature in the governance of education systems. We start with a brief explanation of 'governance' and how hierarchies, markets and networks have featured in the coordination of education systems over the years. We then present the main questions for this chapter before we continue to outline each of the three archetypes separately in more detail.

Governance, according to Windzio et al. (2005), is a specific form of coordination of social actions characterized by institutionalized, binding regulations and enduring patterns of interaction. These forms vary from institutionalized self-regulation of civil societal elements on the one hand, to authoritative decision-making by governmental actors on the other, with an intermediate range including cooperation of governmental, private and various collective actors. 
The three main forms of governance of educational systems, according to Windzio et al. (2005), are markets, hierarchy and networks; each has a distinct structure in institutions and actor constellations (e.g. state and/or non-state), and a unique process or mode of social coordination by which actors engage in rule-making and implementation and in the provision of education (Börzel and Risse, 2010).

Hierarchical control dominated most post-war Western European education systems until the 1980s, according to Greany and Higham (2018). This style of governance, based on authority, clear division of tasks, rules, rationality and objectivity was partly replaced by market-based types of coordination in the 1980s where choice, a focus on price, efficiency and decentralization were seen as the best drivers for quality and improvement. The limitations of marketized systems, particularly for disadvantaged students and parents, led amongst other reasons to the emergence of network governance in the 1990s. Here, interdependence, trust and empathy are the organizing principles where school-to-school collaboration is seen as a viable strategy for improvement or where community-based partnerships between schools and other service providers are expected to address high inequality in the education system. The OECD (2012) emphasizes that such collaboration and the relationships between schools, parents and communities particularly matter in addressing inequality. It expects such partnerships to prevent drop-out of vulnerable children and optimize their learning, such as by improving the transition of children between school phases or by improving parental involvement in schools. A review by Aldridge (2004) highlights how cohesive communities and strong educational networks can provide safety nets and opportunities for disadvantaged children, and as such promote upward social mobility and increase children's life chances.

This chapter explores in more detail how each type of coordination has a different set of accountability relations and structures with implications for how capacity is build and (re)distributed and whether and how people come or need to trust one another. The description of hierarchy, market and network governance next follows the pattern of setting them forth as ideal types, allowing us to organize our thinking about the role of trust, accountability and capacity in each of the three types. In reality, many education systems however have a combination of two or three of these modes which means that our argument is mostly conceptual where examples are presented only for illustrative purposes to understand:

1. How does hierarchical, market-based and network governance aim to build capacity across an education system, and whose capacity and how?

2. How is accountability typically organized in each of the three types of systems?

3. What set of relations underpin each type of governance and how are hightrust relations and trust in the education system relevant conditions for capacity-building in each of the three systems? 
We'll next address these questions for the three types of systems separately. Each section starts with a description of the particular governance style, the relations through which the provision of education is typically coordinated and regulated and the dominant model of accountability. We then move to explain how each system assumes to build capacity for high teaching and learning, the unintended consequences we typically find in each system and how these might reduce its overall effectiveness. Throughout our discussion we reflect on the role of trust in the coordination and accountability of various actors and how trust is, or is not, a necessity for a well-functioning system.

\section{Hierarchy}

Hierarchical education systems are those which are tightly managed from the centre with strong, top-down control of recruitment, promotion, curriculum and content of classroom instruction (Levy et al., 2018). The relations in such systems are typically 'command and control' with a high level of dependence where the state regulates school quality on the basis of a set of national standards and is responsible for most of a school's daily operations. Hierarchical education systems were central to the development of the post-war world order, according to Robertson (2012), with strong states at the national or subnational level. Windzio et al. (2005) present the former German Democratic Republic as an example of a pure centralist governance form where the state hierarchically controlled and predetermined the actions of its teachers who could only organize themselves in state-controlled unions. This type of governance was highly capable of reaching collective decisions, according to these authors, but allowed little flexibility to respond to individual student needs as we'll argue next.

Pritchett (2015, p. 15) portrays the relations in a hierarchy as those of 'principal-agent' where schooling is delegated to agents (e.g. teachers, schools) who act on behalf of a principal (e.g. the state, ministry of education). The main actors in this set of relations are typically:

Citizens/Parents/Students: those who (1) ultimately control the sovereign, (2) are the direct participants and intended beneficiaries of instructional services via schooling and (3) those under whose objectives the outcomes of the system are normatively evaluated (both individually and collectively).

The Executive Apparatus of the State: the actors who control, via executive decisions like the adoption of laws, regulations, policies and the allocation of budgets, the apparatus of the state. This can, according to Pritchett (2015), be either a nation-state or, in a federal system, the tier of government with primary responsibility for schooling, both politicians and executive policymakers.

Organizational providers of schooling: schools and organizations (like Ministries of Education) that control all aspects of the operation of schools through a large bureaucratic structure.

Teachers: individuals, or direct 'front-line service providers' of instructional services. 
Hierarchies, in their pure form, have a public or state model of accountability and control, according to De Grauwe (2007, pp. 14-16). In this model, employers or political power holders are in charge of monitoring: teachers are controlled by school head teachers, who are controlled by district officers, who are controlled by central ministries that in turn are directed by elected representatives. This monitoring system has democratic legitimacy because of the control chain that emanates from the political level. In this model, external forms of quality monitoring tend to prevail over internal ones, and the traditional inspection system, which can be more or less decentralized, will play a key role in motivating school improvement, according to De Grauwe (2007).

Pritchett (2015) takes a somewhat broader view in describing the accountability in hierarchical systems. He refers to four design elements through which the relationships of accountability function: delegation, finance, information and motivation. These elements include not just the monitoring and evaluation with incentives for change (which is how we often understand 'accountability'), but also how resources are distributed across the system. Delegation is where the principal specifies objectives and goals and 'what they want done' to the agent. An example would be a Ministry of Education setting a national curriculum and set of performance targets that school principals have to implement and adhere to. Finances are provided up front to implement such objectives. Information and motivation further incentivize the performance of the agent towards the principal's aims, for instance through an inspection that monitors the implementation of a national curriculum. Information, such as the assessments from inspections, allows the principal to decide on whether the agent's outcomes and performance meet the set objective, where a set of intrinsic or extrinsic (pecuniary) motivators will inform what happens when objectives are not met. Altrichter et al. (2015) describe the consequences of eight European inspection systems which are as varied as naming and shaming low-performing schools to replacing head teachers of failing schools.

Following Pritchett's (2015, p. 18) argument, well-functioning hierarchies are those where there is an adequate flow of accountability in the system where principal and agents work towards the same objectives. In the best possible case, politics works well so that citizen/parental demand for education is made a salient issue for politicians and their top policymakers, the executive apparatus of the state is able to convey these effectively to schools, schools are able to create the conditions in which teachers can work well, citizens and parents can also participate locally and directly and high-quality instructional services of the types that are contextually relevant are the result. In this case, the four design elements we previously referred to (delegation, finance, information and motivation) are coherent which may either build or restrict capacity for improvement, something we'll next discuss in more detail.

Standardization (and alignment around those standards) is the main principle of hierarchical coordination, according to Freidson (2001). Hierarchical systems are expected to work well where production can be standardized and where the 
monitorability of outputs and/or outcomes is straightforward. The execution of bureaucratic tasks, such as the dispensation of textbooks or the building of schools, would be typical examples for education systems (Levy, 2018), Freidson (2001) argues that hierarchies champion a mechanistic scientific model where organizations function as a machine according to a highly routinized process of work and division of labour, where government is the key player in prescribing such processes and tasks through top-down decision-making. In such models, education and high-quality teaching are seen as tasks that can be standardized and regulated from the top and compliance of educators follows from a system of rules which establish the duties of each position, occupation or job as well as their relationship (Levy, 2018).

The implementation and monitoring of the national curriculum in South Africa provides a good example of how hierarchical systems aim to improve education through the standardization of teaching and learning. Here, teachers are provided with an annual teaching plan which sets out detailed guidelines on the content that needs to be taught each week and the activities students need to complete. Monitoring by the district is put in place to ensure compliance to these prescriptions.

The rule-based nature of such arrangements, where agents are, as in the South African example, expected to follow a system of rules, does not necessarily value trust as an important principle for the functioning of such systems. Tyler (1990) has however argued that general trust in government would promote voluntary compliance of those the government aims to regulate. Their work suggests that trust in government leads to large-scale consent with government regulation, reducing the need to enforce compliance through frequent monitoring and control. In the example of South Africa, we would expect teachers to implement the curriculum as prescribed when they trust their government to provide them with relevant and legitimate guidelines. In this case, general or system trust acts as a mechanism to converge objectives. Ferris (1992) explains how similarity of objectives between principals and agents reduces the need for intense monitoring as principals (e.g. the Ministry of Education) will have little reason to be concerned over potential opportunistic behaviour of agents, in this case teachers.

Opponents of hierarchical systems however argue that educational outputs are more heterogeneous and cannot easily be captured in a set of standards and objectives. Robertson (2012) and Looney (2009) for example argue that teaching requires a level of professionalism which doesn't sit well with a high level of standardization. Robertson (2012, p. 590) refers to an ILO/UNESCO report which states that 'teaching is a form of public service which requires of teachers expert knowledge and specialized skills, acquired and maintained through rigorous and continuing study'. Teachers should enjoy academic freedom in the discharge of professional duties to include the choice and adaptation of teaching material and pedagogy; only when they have a degree of autonomy and flexibility can they improve performance of students with various learning needs and backgrounds. Standardization is, according to Looney (2009), at odds with 
supporting individual differences and interests and, although it would provide a fair and equal teaching offer to all, it doesn't value students for their individual needs and learning styles. Our example from South Africa provides a case in point. A principal in Paterson et al.'s (in prep.) case study work explains how the annual teaching plan, prescribed by government, prevents teachers from reteaching content to students who failed to understand it in the first round of instruction:

And another challenge that is there is this one - you know with our ATPs (annual teaching plan) and the curriculum, like the CAPS ... we're told in workshops - teach ... if the kids did not understand, re-teach, re-assess. You see ... however in the ATP there is no time for that.

The example highlights how hierarchical control, by definition, reduces teachers' flexibility to adapt their work to local context and needs. As Freidson (2001) argues, when the logic of a bureaucracy is fully developed, professional tasks that require discretion based on schooled knowledge as well as experience are transformed into a standard set of routines which fail to take into account individual human needs. The reduction of professional agency also features in Hadfield and Ainscow's (2018) work in in Wales. They describe how the strong hierarchy there created a professional context where the majority of school leaders deferred to those in higher positions and, when conflict arose, tended to resist passively rather than actively. Following Freidson's (2001) argument, in a hierarchy 'workers' tend to be particularly motivated by their desire to hold on to their job and their prospects within the school, rather than by their commitment to any particular kind of work.

How does the high level of standardization and reduction of professional agency affect trust, and particularly trust between students and teachers? Here we refer back to our definition of interpersonal trust in Chapter 1 and the three elements in our trust definition:

- Competence: the perceived ability, or expectation that the other party has competence to successfully complete its task.

- Benevolence: the expectation that the other party cares about the trustor's interests and needs.

- Integrity: the expectation that the other party will act in a just and fair way.

The rationale for a high level of standardization is to maximize the predictability and reliability of services and prevent any 'favouritism' or preferential treatment of students. This would support the integrity aspect of the trust relation. However, the lack of commitment to individual student learning and/or lack of opportunity to appropriately serve those students who fall outside of the norm would likely violate the competence and benevolence aspects of our trust definition.

Hierarchical systems with their high level of standardization along vertical lines of coordination and accountability thus seem mostly relevant for simple 
bureaucratic tasks, but fail to deliver more heterogeneous outputs that require a high degree of professionalism. We briefly touched on how trust in government would support compliance to centralized regulation, but how a high level of standardization potentially reduces interpersonal trust, such as between students and teachers. Our earlier reference to alignment however provides a counterargument which we develop next. We'll first explain what we mean by alignment in hierarchies and how it affects capacity-building and trust in the system. As we'll see in our next discussion, the argument is not straightforward as strong alignment can both build and reduce capacity and trust. We expect the accountability context, initial levels of teacher professionalism and perceived fairness of standards to inform the direction of the effect and the specific patterns of causality.

\section{A further note on alignment}

Alignment or 'coherence' is what makes a hierarchy functioning, according to Pritchett (2015). In such systems, delegation, finance, information and motivation are coherent within and across the various accountability relations. All the actors have similar information to act on, a similar set of standards to work towards and are rewarded for activities that would raise student learning. Technical alignment or coherence ensures that the measurement of behaviour and performance of students and educators alike is consistent across levels and over time while the incentives to improve performance motivate people to learn an develop towards a common point of reference. Technical alignment between performance standards, curriculum, assessment and evaluation allows policymakers and practitioners to have appropriate data in the right time frame to meet their decision-making needs, according to Looney (2011). Standards allow accountability and feedback systems to provide consistent information across the system to support learning and adaptation, particularly where those standards incentivize actors the system to improve outcomes.

In a well-aligned or cohesive system, the exo and macro-level are organized around a common set of standards and indicators so that various elements and actors all work towards the same goals of high learning outcomes and organize the educational processes towards those outcomes. In such systems, learning is articulated as an objective in the relation from the state to organizational providers of schooling (e.g. schools or other service providers) and to teachers, where information is gathered about learning and the relationships are managed on the basis of this information.

Typical examples of limited alignment are provided by Pritchett (2015) who talks about delegation being too overambitious relative to the amount or structure of how schools are financed, or too vague for regular reliable and relevant information on performance to be created. In incoherent systems, principals may have objectives that are completely different to goals of the Ministry of Education and how they manage teachers, or there may be policies and goals to improve 
student outcomes but teachers are promoted on the basis of seniority, or when the magnitude of finance made available (both in terms of wages and resources for inputs) is incompatible with what teachers are being asked to accomplish and are held accountable for. As we explained before, these inconsistencies will reduce capacity when actors have to negotiate conflicting demands and are managed ineffectively with contradictory standards and lines of accountability.

Holding teachers accountable for standards without providing them with resources to implement these standards is also a guarantee for trust-relations to break down. When people and organizations are held accountable on standards and targets for which their counterpart needs, but fails to deliver resources, this creates a sense of unfairness and potential opposition to change.

Strong alignment of standards across the system thus has the potential to improve trust. We refer to Ehren (2016) who talks about a mechanism of 'setting expectations'. Strong alignment, when creating a common and shared understanding of values and quality, would allow educators to develop high-trust relations as there is less need to reconcile potential opposing views and parents and students would also know what to expect. The argument is grounded in discussions about antecedents of trust and how people come to trust each other. Morrone et al. (2009) and Uslaner (2008) for example identify expectations and the belief that most people share your moral values as central to most definitions of trust, where Hardin (2004; in OECD, 2017) argues that trust is conditional on a set of beliefs about the person being trusted and on their likely behaviour with respect to a given activity. A clear and well-aligned set of roles, responsibilities and accountability standards will create a solid and shared expectation of what good behaviour looks like, and will allow teachers, head teachers, parents and students to refer to these shared standards when evaluating and addressing their counterpart's behaviour. Such shared standards may even mitigate the breakdown of trust between students and teachers we described earlier, particularly when students (and their parents) are no longer expect to be taught individually.

Whether this is in their best interest is another matter and reflects a similar point made by Levy (2018). He talks about hierarchical systems getting stuck in a low-level equilibrium when organized around schooling and process compliance and not around learning outcomes (see Pritchett, 2015). Levy (2018) and Pritchett (2015) assert that many hierarchical systems, particularly in developing countries, have policies that favour form over function and have emphasized the expansion of schooling, rather than actual learning. Examples are of governments who have procedures for hiring teachers on the basis of their qualifications, or tracking enrolment over time, across space, and by grade without linking those processes to student outcomes. These systems have been successful expanding both the fraction of all children exposed to schooling as well increasing the duration of exposure per child, but have failed to actually improve learning. The lack of performance information locks the system in an equilibrium and creates what Robert Michels described as goal displacement; a shift from ends, or ultimate goals, to means, or instrumental goals. It is these inefficiencies that have caused 
various governments to introduce market mechanisms into education, as we'1l explain in the next section.

\section{Markets}

Market governance is the opposite of hierarchy in that it constitutes a set of independent relations between 'buyer' and 'seller' and other societal actors involved in education, rather than the dependent relations that underpin hierarchical systems. Market governance in education is a response to the stalled economic growth in the 1960s and 1970s, according to Robertson (2012), which led to the emergence of new policies promoting deregulation, competitiveness and privatization of schooling. At the time, price and competition were thought to be more effective ways to coordinate the delivery of public services in general, compared with central regulation and control.

Many governments have introduced market mechanisms in education in the last three decennia, according to Waslander et al. (2010). They have done so by enhancing parental choice and encouraging school competition, through policies like abolishing catchment areas, creating voucher programmes and setting up charter schools. Greany and Earley (2017) present the example of England in the 1980s and 1990s as a typical example of a (quasi)market-based system where parental school choice, privatization and market rhetoric dominated the system, and schools can buy improvement services on an open and transparent market place. How are we to understand a focus on competition and choice, and what are the implications for teachers and students who are located in national settings but where power is now concentrated in spaces not open to political contestation?

Greany and Earley (2017) argue that the rationale for quasi-market systems reflect a neoliberal consensus of education as investing in human capital and the improvement of one's personal and national competitiveness in a global economy. Teachers' pedagogic practices are presumed to materialize the competent learner for a knowledge-based economy, instead of ensuring the acquisition of those knowledges that may not be directly useful for the economy or offering a chance of personal fulfilment. Where hierarchical systems value education for its value for contributing to a robust democracy by transmitting values, beliefs and skills that support and maintain a society or culture or common sense of a 'public' (see Robertson, 2012; Pritchett, 2015), markets treat education as a commodity that can be bought and sold. Rather than valuing education as a citizen right, it is now defined as a private good where quality is coordinated through price, supply and demand.

De Grauwe (2007) and Whitty et al. (1998) explain that advocates of quasimarkets argue that competition and choice will lead to increased diversity of provision, better and more efficient management of schools and enhanced professionalism and school effectiveness. Marketization is expected to bring particular benefits for families from disadvantaged communities, who have been ill-served 
by more conventional arrangements. The justifying belief is one of superiority of the private sector in driving up standards, compared with public institutions.

The accountability in market-based systems is typically designed to support decision-making of 'customers', competition and choice processes in what De Grauwe (2007) calls 'consumerist' or 'free market' models of accountability. In the consumerist model, the main actors in charge of monitoring are supposed to be the consumers or beneficiaries of the education system, i.e. the students, the parents and the wider community. The free market model of accountability intends to break away from public control and to replace it with the control of the individual consumer. The ultimate way of moving from public control to market control is to provide parents with vouchers, allowing them to buy the education they want for their children and put schools in a competitive position. In that case, individual family demand would become the regulating principle for education development in replacement of public control. Market-based systems typically also have some state-level accountability of performance indicators and rakings to inform school choice, driving up student performance and ensuring that teachers work more efficiently, according to Waslander et al. (2010) and Robertson (2012); high stakes testing would allow governments to publish league tables of schools' performance where parents are expected to send their child to the schools with the highest scores, motivating those schools with lower performance to either improve their scores or close when student numbers go down.

Markets reposition students and their parents as informed and rational consumers of private goods, where teachers deliver services in response to their demands. Such hard-edged economic emphasis on individuals, flexibility, competition and incentives is likely to undermine the basis of learning, according to Robertson (2012). She argues that the social encounter between teacher and student dramatically changes when the first is now positioned as simply there to improve student performance as efficiently as possible, instead of as individuals with desires and passions to make a difference in the lives of students.

In an ideal market, students (or their parents acting on their behalf) are well-informed consumers or 'buyers' and teachers the 'sellers' of knowledge and instruction. The assumption is that students can exit the relationship with their teacher and school when the expected service level is not delivered and that they are capable of evaluating the quality of education, using available external accountability information to inform their decision.

Such 'buyer-seller' relations and the role of trust in enabling effective transactions are frequently discussed in management literature, such as by Dyer and Chu (2003), Gundlach and Cannon (2010) or Vanneste and Frank (2013). These authors talk about economic efficiency of high-trust interfirm transactions between buyers and suppliers, positioning either buyer or supplier as the trustor or referent of trust, depending on who is most vulnerable in the exchange relationship. Vulnerability would be associated with potential opportunistic behaviour of the counterpart in the relation (e.g. when stealing ideas 
or misusing confidential information to one's own competitive advantage), or with a suboptimal delivery of products of services. Trust between buyer and supplier, according to these authors, is expected to reduce transaction costs and eliminate the need for formal contracts, which are costly to write, monitor and enforce. A buyer's trust that a supplier will perform would yield economic efficiencies through mitigating transaction costs that would otherwise occur in the absence of trust, such as when a buyer has to make detailed specifications on the type of product the supplier delivers and has to frequently monitor the terms of the agreement.

When we apply these understandings to an education context, we would expect that high trust between students and parents on the one hand (as buyers) and teachers and the principal on the other (as suppliers) would reduce the need for detailed agreements which for example outline the home-school partnership, or set out parental and school responsibilities in educating children. When there is no need for such contracts, students and their parents work in partnership with the teacher and school to ensure a good-quality education.

The management literature also positions the economic advantage of trust in terms of sharing of information. In a high-trust exchange relationship, where the supplier can trust the buyer not to behave opportunistically, the supplier will be more willing to share confidential information. Sharing of information on costs or new ideas and technologies allows both partners to reduce production costs and improve product design and process innovations. A lack of trust, such as when the supplier expects the buyer to steal its ideas or share them with competitors, may cause suppliers to suppress potentially relevant information that would be useful for problem solving.

This chapter however seems less applicable for education where the intangibility of teaching and the lack of a pure market place would present little, or perhaps other risks for sharing of information between students and teachers to improve learning outcomes. Teachers are generally well protected by labour laws which would reduce their anxiety over sharing some of their best practices. Even more important, the relation between students and teachers is essentially one of co-production where students need to be actively engaged in the delivery of the service (the teaching) to ensure the economic advantage of the exchange. Information sharing is inherent to the transaction, and without it, there will not be a transaction at all.

Vanneste and Frank's (2013) reference to relational contracts, compared with formal contracts for the delivery of physical goods, is more relevant here. Relational contracts refer to a service or other intangible outcomes of a relationship which cannot be enforced by outsiders. Considering the relation between a teacher and his/her student, we can immediately see how an outsider (such as the principal or external inspector) would struggle to control every element of their interaction. Not only would such pervasive external control change the very nature of the relationship to the type of narrow standardized responses discussed in the previous section, the heterogeneous nature of education, where teachers 
need a degree of professional discretion to meet individual learning needs, also makes such monitoring highly contentious.

Positioning the exchange between student/parent and teacher/principal as a relational contract suggests that particularly a shared history, expected future interactions and potential alternatives and 'outside options' would inform the effectiveness of the relationship and whether parents/students would terminate the relationship. Vanneste and Frank (2013) argue that a shared history facilitates social interactions, which in turn can lead to the establishment of cooperative norms such as trust; the development of such norms is more likely with the view of a continuation of the relationship where high trust facilitates mechanisms associated with 'voice' (i.e. joint problem solving) rather than exit (termination of the relationship). In an educational context, we would thus expect that students/parents, and teachers/principals develop a shared understanding of the value of education and specific goals of teaching over time, where high trust allows them to successfully negotiate opposing views and where sensitive information is shared to ensure high learning outcomes. The evidence of collective trust in schools, presented in Chapter 1, would support this argument.

Various scholars however argue that market mechanisms are more likely to break trust, particularly of disadvantaged students and parents. Marketized systems, by their nature, incentivize schools to respond to customer demand and compete with other schools for the most desirable 'clientele'. The least desirable students are, according to Whitty et al. (1998), those who are 'less able', have special educational needs, especially emotional and behavioural difficulties, as well as children from working class backgrounds and boys, unless they also have some of the more desirable attributes. The more able students stay in the system longer and thus bring in more money, as well as making the school appear successful in terms of its test scores and hence attractive to other desirable clients. Whitty et al. (1998) argue that, given the opportunity, most schools will want to become more selective because taking children who will bring scores down will affect their overall market position. The rationality of choice and competition thus typically only works for students from advantaged background who (or whose parents) are able to navigate the system to their advantage. Leaners who require 'more work' will likely feel disrespected by the system and lack trust in teachers and schools who either don't provide them with opportunities to progress or subject them to a high-stress teaching and learning environment.

Competition also seems to strain trust relations between principals and teachers in schools. MacBeath (2012) talks about a damaging effect of neoliberal policies on teachers' collaborative work as there are little incentives for more socially organized encounters. Weenink (2009) argues that marketization creates different value systems between teachers and principals that are often at conflict with one another. His study in the Netherlands shows how a school management's response of attracting able students and their willingness to raise fees was at odds with teachers' commitment to social justice. Particularly principals who face high competition from other schools tend to invest school resources into promotion 
and marketing or introduce practices and programmes that are likely to attract high-ability students, such as strict selection policies to attract high-ability students, or introduce costly school uniforms (Teelken et al., 2005; Lubienski, 2009), rather than promoting the learning and development of a wider student population. This ideological gap potentially breaks trust between teachers and principals but may equally break trust between teachers and students when the latter questions the benevolence and integrity of the school and teachers.

The risk of such tensions depends on the position of schools in the local community according to Waslander et al. (2010) and Greany and Higham (2018). These authors talk about local (informal) rankings of schools where schools at the top will feel less need to engage in the market and respond strategically and will have fewer disputes over a market mode or professional model of conduct. These rankings are, according to Greany and Higham (2018), informed by schools' status and popularity with parents, where the school's status is a reflection of its history, student intake, aggressive marketing campaign and inspection grade. Interviewees in their study talk about a context of 'winners and losers', where higher status schools benefit in terms of new opportunities and resources as a result of policy reform, but where lower status schools faced a concentration of challenges often including undersubscription, higher mobility and disproportionate numbers of disadvantaged, migrant and hard to place children. Some schools, particularly in highly competitive areas, turned to 'cream skimming' to recruit a particular type of students to improve the school's popularity in the neighbourhood, while also increasing the chances of doing well in standardized tests, performance league tables and Ofsted inspections. The inspection grade was an important piece of information for parental school choice and structured the competition amongst schools.

Whether market mechanisms can improve education has been the topic of fierce debates. Proponents claim higher quality, efficiency and demand sensitivity where others, such as Darling-Hammond (2004) and Ladd (2007), however also show how marketization leads to higher inequality, segregation and increasingly unequal access to schools. The evidence so far suggests that choice and competition will lead to better quality schooling for some children, particularly the most able and advantaged, but will also increase inequality between schools and lead to, what Whitty et al. (1998) refer to as, working class children and particularly children with special educational needs being 'ghetto-ized' in poorly resourced schools. How choice and competition affect school quality and improvement will vary across level of urbanization and socio-demographic features of the market place. But even in urban areas with many options, parents often don't prefer schools on the basis of their academic outcomes but decide on the basis of other indicators such as proximity to their home (particularly for primary schools). Demographic and socio-economic background factors, level of education and income all play an important role in parental choice processes, where parents tend to prefer schools with a certain composition of the student and teacher population, climate and safety, curriculum or pedagogical orientation (Bell, 2005). 
A general pattern underlying school choice seems to be that parents who are well educated, well-off and involved in their children's education are overrepresented in the group of active choosers (Waslander et al., 2010) where white parents more often try to avoid schools with high proportions of minority and lowincome students, while minority and religious groups may opt for schools with high representation of their groups as well. If the local school can provide high (perceived) quality, a sense of communication and collaboration with parents and overall satisfaction, then the likelihood that parents choose another school diminishes.

Market mechanisms operate in various ways with diverging consequences, depending on specific push-and-pull factors of the local context and actual competition and school choice. High trust, particularly between schools and educators in a local community, may mitigate some of the unintended consequences of market-based mechanisms, but the organizing principle of choice and competition would rule out the role of trust as a lever for learning and improvement. Our final archetype of network governance however is based on trust and provides a counter narrative.

\section{Networks}

Network governance emerged in the 1990s as a new type of coordination, based on interdependency, trust and empathy (Meuleman, 2011). It was a response to both the dramatic intensification of social complexity and a recognition of the failure of both hierarchy and marketization, according to Grimaldi (2011). Network-like organizational forms are thought to be more effective tools of governance in enacting processes of innovation, improvement and self-organization. In education, network governance has been promoted by various international agencies (particularly the OECD), according to Grimaldi (2011), as an effective scenario for tackling major educational issues, such as persistent academic underperformance, students' transition from school to work or childhood obesity. Creating an interconnected approach to, and comprehensive offer of, social, educational and health services through community-based partnerships is expected to more effectively address complex educational challenges in child development (Díaz-Gibson et al., 2014).

For a time, network governance was considered to be a hybrid form of hierarchy and market, according to Meuleman (2008), but it is now seen as a separate third style. Collaboration between interdependent actors is the main principle of coordination where actors are involved in decision-making and jointly coordinate policy implementation. It is considered most effective when provision cannot be standardized and local actors need a degree of autonomy and flexibility to coordinate their work. The outcomes of such collaboration can be multifarious but will, by the nature of network governance, transcend each individual member organization. Provan and Kenis (2008, p. 230) talk about 'network level outcomes' that could not normally be achieved by individual 
organizational participants acting independently'. Such outcomes are categorized by Gray et al. (2003) with specific examples for educational networks by Ehren and Perryman (2018):

1. Creating synergy: adding value by combining mutually reinforcing interests. Synergy is created when the partnership adds value by combining mutually reinforcing interests, such as when students benefit from a broader curriculum range, have access to more facilities or when schools share professional development.

2. Leading to transformation: transform different views into an ideological consensus, such as when all schools in a region sign up to the same model of teaching and behavioural policy and high expectations of student outcomes.

3. Enhancing financial efficiency: maximizing the use of resources across the partners in the network; e.g. when schools share IT or HR services, or use school and classroom infrastructure across a number of schools.

Network governance is premised on local outcomes and capacity-building where outcomes will likely vary across regions, districts, municipalities and provinces, depending on the nature of the issues each network aims to address and the extent to which the network has the autonomy to set its own goals and structures of collaboration. In some countries, networks have been part of governmentinitiated reforms where national policies and regulations have either incentivized or mandated specific types of collaboration and outcomes.

In West Belfast, we find an example of the first where area-based learning communities of primary and post-primary schools collaborate to provide a broad and balanced curriculum and to deliver on the requirements of the 'Entitlement Framework'. The Entitlement Framework requires schools to provide pupils with access to a minimum number of courses at Key Stage 4 (24 courses) and a minimum number of courses at post-16 (27 courses). To reach these targets, Article 21 of the Education Order 2006 enables schools to secure course provision for students at other institutions within the area learning community. Thus, a key purpose of these networks is to allow the level of flexibility required to enable students to take courses at more than one institution (see Brown et al., 2020).

The Netherlands offers an example of mandated networks for inclusive education. Here, national government introduced new legislation in 2014 which required mainstream schools and special schools to work in partnerships under a new authority to provide inclusive education for all children (including children with disabilities) in a region. These networks were centrally formed by the Ministry of Education, Culture and Science according to the geographical location of schools, number of pupils, existing informal cooperation between schools and after consultation with the school boards. Each network of mainstream and special needs schools is governed by new education authorities who are responsible for ensuring close collaboration between these schools in the provision of care and high-quality education to each pupil. They have a legal 'duty to care' 
which means that they are formally responsible for finding an adequate school place for each pupil in their area where children with special needs are supported in mainstream education as much as possible. The collaboration of mainstream and special schools is thought to enhance the learning and overall well-being of children, particularly those with disabilities. A network approach would allow these schools to be more responsive to their needs.

Other examples of voluntary networks, such as peer review partnerships in England (e.g. Challenge Partners), have a much more fluid structure. Here, members organize themselves according to common interests where the network dissolves when there is no longer a need to collaborate or schools join other more opportune partnerships.

Mandated versus voluntary networks are each premised on a different idea about how best to innovate schools and educational systems and how to build capacity for improvement, according to Grimaldi (2011). Mandated networks or those incentivized by national government particularly represent a rationale of management and control, allowing the transfer and spreading of standardized knowledges and practices. Voluntary networks on the other hand can be understood as an organizational configuration emerging from the bottom, being it planned or spontaneous, that develops through authentic peer-to-peer and self-organizing logics. Grimaldi (2011, p. 145) argues that in the first case, networks follow a technical rationality, based on the idea that general solutions to situated and practical problems can be developed outside the contexts of those practices. Such a network model is often associated with the logic of best practices transfer within failing and disadvantaged contexts. This model of 'rescue intervention' underpins some of the Multi-Academy Trusts in England, particularly those that have been asked by representatives of the Department for Education (Regional Schools Commissioners) to take on failing schools. These schools, which have failed their Ofsted inspection, can be mandated to convert from being governed by their local authority to academy-status, where they are placed under the governance of a high-performing trust. The 'rebrokering' is expected to drive up standards in the failing school when the trust board implements its tested and successful strategies in the school. Grimaldi (2011) argues that such models, contrary to more decentralized and voluntary types of networks, do not generate or build trust given that the process of labelling and assigning negative identities to schools and school staff that 'need to be rescued' produces a coercive transfer of practices, languages, definitions, concepts and solutions from one context to another and establishes a hierarchical order between the 'rescuer' and 'rescued'. Greany and Higham (2018) therefore position this model as 'merger and acquisition' with features of a hierarchy, instead of pure networks.

In the second case of voluntary networks, the inspiring rationality is one of the dialogues, according to Grimaldi (2011), allowing for symmetrical relations within the network which are structured by processes of exchange (comparison, influence, learning and so on), a reciprocal recognition and understanding. Here partners join out of a common interest and develop trust over time when both 
spontaneous and formal relations develop, and norms on how to collaborate and which outcomes to work towards are established.

Capacity is an important precursor for effective network governance. Hadfield and Ainscow (2018) explain how a lack of structures and processes for collaborative school improvement was one of the most important reasons why regional consortia in Wales failed. These consortia struggled to build local capacity for improvement, due to an absence of sufficient interlocking networks or subsystems of school leaders and groups of schools to deal with a region's systemic school improvement issues. According to these authors, the capacity to collaborate was evident in some areas but not in others. The gap was too great to allow the collaborative model to translate into a system-wide approach of network governance. According to Hadfield and Ainscow (2018), network governance requires an intermediate organization that can facilitate system-level transition and broker collaboration between local partners, develop capacity for leadership at all levels of the system and create and maintain a strong political mandate for a collaborative local approach, both at the national and local level.

The conditions that assure an effective functioning of networks are complex, and organizational forms are widely varied in participants' number, typology, scale and objective. Grimaldi (2011, p. 147) explains how motivation, commitment and trust among partners cannot be taken for granted, and all of these factors are crucial to the enactment of collaborative practices. The features of a network's design, the modalities through which they are created and the objectives pursued all need to be analysed carefully to understand how network governance can improve education.

Following Kenis and Provan's work on organizational networks in other sectors (e.g. health, youth services), the size of networks, the geographical location of members and trust between partners appear to be relevant conditions for high outcomes. Their work suggests that there is an optimum structure where larger, more dispersed networks need more brokered forms of governance where a separate administrative entity or one member organization governs the network. Large networks, according to these authors, will struggle to have effective forms of bottom-up shared governance as members will either ignore critical network issues or spend significant amounts of time trying to coordinate large numbers of organizations, particularly when participants are geographically dispersed. Larger networks often also face problems with the distribution of trust across the network and with ensuring goal consensus. Examples of such brokered networks are the teaching school alliances in England which function to support professional development, school improvement, leadership development and initial teacher training of their member schools. Each alliance is led by one of their members who meet the government's eligibility criteria (amongst others an outstanding judgement by Ofsted, the Inspectorate of Education) and have been approved as a 'teaching school'. Where networks are small, members are geographically close and trust is pervasive throughout the network, there is less need for a management role, and shared, bottom-up collaboration is most likely to be 
equally effective in improving learning and development across the network and student outcomes.

Support for this contingency model for educational networks comes from small case study work by Ehren and Godfrey (2017) who found that large national Multi-Academy Trusts, after an initial start-up phase, often set up regional hubs to coordinate the collaboration between schools more locally as a way to improve their span of control. These hubs have a mix of high and low-performing schools to distribute the improvement capacity across the portfolio. In the Netherlands, the work of Svensson (in Ehren, 2016) furthermore shows how a lack of trust between school boards in networks for inclusive education, caused by a past history of competition and divergent views on education, inhibited their collaboration. School boards decided to develop as little partnership as possible, by distributing resources directly to each school board; they never really integrated their provision.

The examples indicate how network governance can also have unintended consequences which may reduce capacity for improvement. Ehren and Perryman (2018, p. 7/8) build on the work of Mayne et al. (2003) to summarize such potential side effects for educational networks:

- Diffusion of roles and responsibilities with limited clarity over where to complain or who to approach when things go wrong. Hutchings et al. (2014) for example explain how parents have little clarity over who is in charge of Multi-Academy Trusts and who to address with concerns.

- Competition between partners in the network over delivery of services when partners are trying to deliver different services to the same clientele and have some home-based loyalties and preferential knowledge in delivering those services. As Gray et al. (2003) explain, stakeholders drawn into collaborative arrangements rarely relinquish their own organizational agendas for the collective good of the network but rather battle to impose their own values on the overall goals of the enterprise. Greany and Ehren (2016) report similar issues for Multi-Academy Trusts in England, where schools who join the Trust are used to 'running their own ship' and oppose working in matrix-like structures where finance, human resources and marketing professionals are employed by the Trust, and where school leaders have to adhere to and work within the framework set by these centralized services. This creates tensions which need to be resolved for the partnership to be effective.

- Issues around service users who do not want to be associated with other users served by the same network organization, such as when pupils going to mainstream schools do not want to be identified with students from a special needs school, or when head teachers of high-performing schools do not want to be associated with a failing school in the network.

- Challenges in having multiple partners in the delivery of services determine success criteria for their services and potential conflicts, coordination 
difficulties and costs, management complexities and power issues about deciding on, and reaching, common goals. Greany and Ehren (2016) for example talk about head teachers and teachers feeling disempowered by having to work within centralized structures of scripted curricula and lesson plans, particularly in large Multi-Academy Trusts where the Chief Executive Officer tries to ensure control over schools through hierarchical governance. Hill (Academies Commission, 2013) notes that the imposition of a centrally mandated model on academies in the Trust reduces school and teacher autonomy and constrains head teachers in developing innovative practices. Some head teachers also report that much of the support from centralized staff goes to schools who are failing to meet the targets set in Ofsted's inspection framework (and are designated as 'requiring improvements' or 'in special measures'), where good and outstanding schools are either expected to fend for themselves or see their resources (e.g. teachers and teaching assistants) taken out of their school to support their poorly performing peers.

- Convergence towards groupthink when members of the network isolate themselves from outside influences and suppress conflicting viewpoints when making decisions. Groupthink is a potential risk where schools in a network are for example expected to implement one and the same organizational model, curriculum and assessment practices. Such centralized decision-making and frameworks potentially create a situation in which member schools are not encouraged to think and act independently and develop innovative solutions for local problems.

- Inefficiencies due to participant turnover and communication and meeting costs, such as when schools leave the network when initial attempts to collaborate fail, or cost too much time.

Network governance, by its nature of involving various actors in decisionmaking and coordinating policy implementation, requires trust between those actors to come to a shared understanding of the problem at hand and a set of relevant activities to address them (Klijn and Koppenjan, 2000). Partners who trust each other will be more willing to collaborate without constant monitoring and checking up on agreements. Trust is thus, according to Bachmann (2001), also a governance mechanism in itself when cooperation flows from informal norms and established social relationships, where dense interpersonal networks provide information as well as opportunities to impose sanctions that are important to the establishment of trust. Such collaboration, when reflecting repeated positive experiences over time will build trust between (groups of) people and organizations, allowing public and (semi-) private actors to link mutual expectations and coordinate actions (Klijn and Koppenjan, 2000). When there is no initial trust between partners, the network is likely to fail as our previous example from the Netherlands shows. The school boards in one of the networks for inclusive education had a history of competition and 
opposing views and values on teaching. Both factors proved unfavourable for constructive collaboration and led to a disintegration of the network into subgroups organized around existing organizational lines and without new forms of collaboration or integration of services.

The shift towards network governance creates, according to Burns and Koster (2016), a complex education landscape with very real questions about which actors at which levels should be held accountable for which outcomes, and how this can function in a coherent and intelligent manner. The tension between tightly controlled accountability mechanisms which seek to minimize risk and error contradicts the fundamental purpose of network governance to allow stakeholders to flexibly respond to local context and collaborate on the basis of trust. Ehren and Perryman (2018) and Ehren et al. (2017) therefore argue for accountability that acknowledges the position of schools within networks and the collaboration between schools towards a common purpose.

Their suggested bottom-up model of accountability includes a qualitative, interpretative and flexible approach of validating good practices of localized and collaborative provision and improvement of education, such as through the use of 'developmental evaluation' or 'participatory evaluation'. In Ehren et al.'s (2017) model, Inspectorates facilitate evaluations which are goal-free, flexible and specific to context and information needs of (a network of) schools and stakeholders. Network members and their stakeholders are involved in all the phases of the accountability exercise, from developing the standards and methods for evaluation to deciding on how to improve network performance and potential consequences for failure. Such an approach allows Inspectorates of Education to engage network members and their stakeholders in making judgements about what type of behaviour is effective and appropriate in which settings and allows for a deep understanding of how networks operate and are effective in solving local problems. Inspectorates facilitate evaluations and inspect against local targets, instead of making judgements on a centralized framework. A localized approach respects the specific purpose of different networks, while simultaneously bringing order to the diffuse and sometimes ambiguous nature of collaborative arrangements.

Ehren et al. (2017) additionally argue that external accountability of networks operating in situations of high uncertainty can only really work if the education system in which they function is characterized by high levels of reciprocal trust. In such a context, accountability becomes a tool for learning and improvement instead of control; dissolving boundaries between schools and other relevant providers in the area, the Inspectorate of Education and policymakers and ensuring the goal of 'good education' is a shared responsibility and endeavour. In such a context, network members can respond quickly to local problems, professionally scrutinize and share local solutions and ensure that school collaboration is not just an 'end in itself' but successfully contributes to improvement of education and student outcomes, even in challenging circumstances. 


\section{Conclusion and discussion}

This chapter provided theoretical insights into trust, accountability and capacity-building in hierarchical education systems, markets and networks. We discussed how hierarchical systems are typically organized around a set of vertical relations of dependency which particularly work well to coordinate the delivery of bureaucratic tasks that are well defined and easy to monitor. More heterogeneous outcomes, such as typical in education systems where students have various learning needs and interests, are more complex to regulate from the top-down, and we explained how doing so potentially breaks interpersonal trust between students and teachers while reducing professional agency of educators and narrowing the set of outcomes in the system to those to which educators are held accountable. Our discussion of alignment of standards, as one of the principles of well-functioning hierarchies, provided a counter narrative in addressing the potential of coherent standardized systems to build trust by aligning expectations and create a set of shared values; one of the antecedents of high-trust relations. Taken to the extreme, strong alignment is however expected to lead to various unintended consequences, such as narrowing the curriculum and teaching to the test, which would reduce trust in similar ways as when standardizing education. Strong alignment, particularly around procedural indicators, is also thought to reduce a system's capacity to improve outcomes as it tends to lock actors into a low-level equilibrium and lead to what has been described as 'goal displacement'.

Market mechanisms are often introduced to offset the inefficiencies of hierarchical systems. They are expected to improve education through competition and choice. We reflected on evidence which indicates that, by definition, market-based systems tend to favour those students and parents who have the capacity and offer of schools to make an informed and rational decision on school choice and can 'vote with their feet' when schools and teachers fail to deliver an agreeable standard of teaching. Competition, fuelled by a school's position on accountability league tables, is known to disservice students with special needs or students who would otherwise compromise a school's position in the market. They would become the 'lost cases' or 'unfavourable client group' in a system of supply and demand where the repositioning of education from a human right and development of individual personality to a commodity and teaching of economically advantageous skills breaks trust of those who are disenfranchized from the system. Where market mechanisms work to build a high standard of education for some, they also tend to create high inequality by leaving vulnerable groups behind.

Network governance was our last ideal-type of coordination, where our brief introduction highlighted the various types of networks that have emerged over the past two decades. We distinguished government-mandated networks which often mirror relations of accountability and trust found in hierarchies, from decentralized voluntary networks that are discussed most often as typical 
examples of network governance. In the latter, trust and a set of interdependent relations are the main tenets of (social) coordination where, as Bachmann (2001) describes, trust can be seen as a coordinating principle in itself. Our examples from Wales and the Netherlands highlighted the role of local capacity and existing levels of trust as conditions for effective networks, such as resources to collaborate and a positive disposition to engage in a partnership with unknown others. Evaluation and accountability can support the development of such partnerships over time, particularly when locking members of the network (e.g. mainstream and special schools and other service providers) into the collaboration and providing them with information to improve their shared work and collective action (e.g. in providing inclusive education in a region). The examples we provided were organized around external inspections, but mature networks also have well-functioning internal mechanisms for information collection and decision-making. These would prevent some of the unintended consequences of networking we discussed, such as high transaction costs, lack of clarity over roles and responsibilities or groupthink.

As systems change their modes of coordination, schools are often faced with a combination of hierarchy, market and network and various, sometimes, contradictory pressures. These combinations can repair some of the flaws inherent in each of the ideal-types, such as a lack of innovation and variety in over-standardized hierarchies, high inequality in (quasi)markets or groupthink, ambiguity and high transaction costs in networks. However, the combination of different modes of governance also provides further sources of failure such as when creating multiple, and potentially conflicting, lines of accountability. Jessop (2002) talks about some of the most prevalent failures of combined models of governance, such as where self-organization needed for network governance rarely materializes when national governments continue to measure and regulate aspects of members' work. How the various systems operate will depend on initial levels of trust, accountability and capacity as we have seen in the examples throughout this chapter. Part 2 of country chapters will present us with more detailed cases, allowing us to understand the specific ways in which the three variables will positively reinforce change or lead to a spiral of decline.

\section{References}

Academies Commission. (2013). Unleashing Greatness: Getting the best from an academised system. London: Academies Commission.

Aldridge, S. (2004). Life Chances and Social Mobility: An Overview of the Evidence. London: Cabinet Office, Prime Ministers' Strategy Unit

Altrichter, Herbert, and David Kemethofer. "Does accountability pressure through school inspections promote school improvement?." School Effectiveness and School Improvement 26, no. 1 (2015): 32-56.

Bachmann, R. (2001).Trust, power and control in trans-organizational relations. Organization Studies, 22(2), 337-365. 
Bell, C. (2005). All Choices Created Equal? How Good Parents Select "Failing” Schools. National Center for the Study of Privatization in Education, Columbia University.

Börzel, T. A., \& Risse, T. (2010). Governance without a state: Can it work?. Regulation $\mathcal{E}$ Governance, 4(2), 113-134.

Brown, M., McNamara, G., O'Hara, J., \& Shevlin, P. (2020). Polycentric inspection: A catalyst for sparking positive interactions in educational networks. Evaluation, 26(1), 76-97.

Burns, T., \& Köster, F. (2016). Governing Education in a Complex World. Educational Research and Innovation. Paris: OECD Publishing.

Darling-Hammond, L. (2004). Standards, accountability, and school reform. Teachers College Record, 106(6), 1047-1085.

De Grauwe, A. (2007). Module 1; Supervision, A Key Component in a Quality Monitoring System. Paris: UNESCO IIEP. http://www.iiep.unesco.org/fileadmin/user_upload/Cap_Dev_ Training/Training_Materials/Supervision/SUP_Mod1.pdf

Díaz-Gibson, J., Civís-Zaragoza, M., \& Guàrdia-Olmos, J. (2014). Strengthening education through collaborative networks: Leading the cultural change, School Leadership $\mathcal{E}$ Management, 34(2), 179-200.

Dyer, J. H., \& Chu, W. (2003). The role of trustworthiness in reducing transaction costs and improving performance: Empirical evidence from the United States, Japan, and Korea. Organization Science, 14(1), 57-68.

Ehren, M. C. M. (Ed.). (2016). Methods and Modalities of Effective School Inspections. Dordrecht: Springer International Publishing.

Ehren, M. C., \& Godfrey, D. (2017). External accountability of collaborative arrangements; a case study of a Multi Academy Trust in England. Educational Assessment, Evaluation and Accountability, 29(4), 339-362

Ehren, M. C., Janssens, F. J. G., Brown, M., McNamara, G., O'Hara, J., \& Shevlin, P. (2017). Evaluation and decentralised governance: Examples of inspections in polycentric education systems. Journal of Educational Change, 18(3), 365-383.

Ehren, M., \& Perryman, J. (2018). Accountability of school networks: Who is accountable to whom and for what?. Educational Management Administration \& Leadership, 46(6), 942-959.

Ferris, J. (1992). School-based decision making: A principal-agent perspective. Educational Evaluation and Policy Analysis, 14(4), 333-346.

Freidson, E. (2001). Professionalism, the Third Logic: On the Practice of Knowledge. Chicago: University of Chicago Press.

Gray, A., Jenkins, B., \& Leeuw, F. (2003). Collaborative Government and Evaluation: The Implications of a New Policy Instrument (p. 1-29). In: A. Gray, B. Jenkins, F. Leeuw, \& J. Mayne (Eds.), Collaboration in Public Services: The Challenge for Evaluation. New Brunswick: Transaction Publishers.

Greany, T., \& P. Earley. (2017). Introduction: School Leadership and Education System Reform. Bloomsbury, 1-13.

Greany,T., \& Ehren,M.C.M. (2016).Written evidence to Education Select Committee inquiry into the performance, accountability and governance of Multi-Academy Trusts. http:// data.parliament.uk/writtenevidence/committeeevidence.svc/evidencedocument/ education-committee/multiacademy-trusts/written/32050.html

Greany, T., \& Higham, R. (2018). Hierarchy, Markets and Networks: Analysing the 'SelfImproving School-led System' Agenda in England and the Implications for Schools. London: IOE Press.

Grimaldi, E. (2011). Governance and Heterachy in Education. Enacting networks for school innovation. Italian Journal of Sociology of Education, 3(2), 114-50. 
Gundlach, G.T., \& Cannon, J. P. (2010). “Trust but verify”? The performance implications of verification strategies in trusting relationships. Journal of the Academy of Marketing Science, 38(4), 399-417.

Hadfield, M., \& Ainscow, M. (2018). Inside a self-improving school system: Collaboration, competition and transition. Journal of Educational Change, 19(4), 441-462.

Hutchings, M., Francis, B., \& de Vries, R. (2014). Chain Effects: The Impact of Academy Chains on Low Income Students. The Sutton Trust.

Jessop, B. (2002). The Future of the Capitalist State. Cambridge: Polity Press.

Klijn, E. H., \& Koppenjan, J. F. (2000). Public management and policy networks: foundations of a network approach to governance. Public Management an International Journal of Research and Theory, 2(2), 135-158.

Ladd, H.F. (2007). Holding Schools Accountable Revisited. 2007 Spencer Foundation Lecture in Education Policy and Management.

Levy, B., Cameron, R., Hoadley, U., \& Naidoo, V. (2018). The politics and governance of basic education: A tale of two South African provinces.

Levy, B. (2018). Improving Basic Education - The Governance Challenge (p.1-27). In: B. Levy, R. Cameron, U. Hoadley, V. Naidoo The Politics and Governance of Basic Education: A Tale of Two South African Provinces. Oxford: Oxford University Press.

Looney,J. (2009). Assessment and innovation in education. OECD Education Working Paper No. 24 (EDU/WKP(2009)3).

Looney, J. (2011). Developing High-Quality Teachers: Teacher evaluation for improvement. European Journal of Education, 46(4), 440-455.

Lubienski, C., Gulosino, C., \& Weitzel, P. (2009), School choice and competitive incentives: Mapping the distribution of educational opportunities across local education markets. American Journal of Education, 115, 601-647.

MacBeath, J. (2012). Future of Teaching Profession. Brussels: Education International.

Mayne, J., Wileman, T., \& Leeuw, F. (2003). Networks and partnering arrangements: New challenges for evaluation and auditing (p. 29-51). In:A. Gray, B. Jenkins, F. Leeuw, J. Mayne (Eds.), Collaboration in Public Services: The Challenge for Evaluation. New Brunswick: Transaction Publishers.

Meuleman, L. (2008). Public Management and the Metagovernance of Hierarchies, Networks and Markets:The Feasibility of Designing and Managing Governance Style Combinations. Dordrecht: Springer Science \& Business Media.

Meuleman, L. (2011). Chapter five metagoverning governance styles-broadening the public manager's action perspective. Interactive Policy Making, Metagovernance, and Democracy, 95, 95-104.

Morrone, A., Tontoranelli, N., \& Ranuzzi, G. (2009). How good is trust? Measuring trust and its role for the progress of societies. OECD Statistics Working Papers, 2009(3), 0_1

OECD (2012). Equity and Quality in Education: Supporting Disadvantaged Students and Schools. Paris: OECD Publishing. doi:10.1787/9789264130852-en

OECD (2017). Key Concepts and Messages Identified in GCES Work; Working Document A Prepared for Strategic Education Governance Advisory Group meeting 18-19 September 2017. Paris: OECD

Pritchett, L. (2015). Creating Education Systems Coherent for Learning Outcomes: Making the Transition from Schooling to Learning. RISE-WP-15/005.

Provan, K. G., \& Kenis, P. (2008). Modes of network governance: Structure, management, and effectiveness. Journal of Public Administration Research and Theory, 18(2), 229-252.

Robertson, S. L. (2012). Placing teachers in global governance agendas. Comparative Education Review, 56(4), 584-607. 


\section{Melanie Ehren and Jacqueline Baxter}

Teelken, C., Driessen, G., \& Smit, F. (2005). Frictions between formal education policy and actual school choice: Case studies in an international comparative perspective. International Review of Education, 51(1), 35-58.

Tyler, T. R. (1990). Why People Obey the Law. New Haven, CT:Yale University Press.

Uslaner, Eric M. "Trust as a moral value.” The handbook of social capital (2008): 101-121. Oxford: Oxford University Press.

Vanneste, B. S., \& Frank, D. H. (2013). Forgiveness in vertical relationships: Incentive and termination effects. Organization Science, 25(6), 1807-1822.

Weenink, D. (2009), Creating a niche in the education market: the rise of internationalised secondary education in the Netherlands. Journal of Education Policy, 24(4), 495-511.

Whitty, G., Power, S., \& Halpin, D. (1998). Devolution and Choice in Education: The School, the State and the Market. Australian Education Review No. 41. Melbourne, VIC, Australia: Australian Council for Educational Research (ACER) Limited.

Waslander, S., Pater, C., \& van der Weide, M. (2010), Markets in education: An analytical review of empirical research on market mechanisms in education. OECD Education Working Papers, No. 52, OECD Publishing. doi: 10.1787/5km4pskmkr27-en

Windzio, M., Sackmann, R., \& Martens, K. (2005). Types of governance in education: a quantitative analysis (No. 25). TranState Working Papers. 


\title{
TRUST-BASED ACCOUNTABILITY IN EDUCATION
}

\section{The role of intrinsic motivation}

\author{
Frédérique Six
}

\section{Introduction}

The central question of this book is how trust, accountability and capacity in/of the school and education system allow teachers to be or become effective. Education systems support teachers to be or become effective when teachers are willing and able to do what is needed to be effective. Willingness relates to teachers' motivation. This chapter focuses on motivation by addressing the question: how can schools and education systems motivate teachers to be(come) effective?

A dominant distinction in motivation theories is located between extrinsic and intrinsic motivation. Self-determination theory (SDT) (for an overview, see Ryan and Deci, 2017) is based on this distinction and has been applied to (at least) the micro-, meso- and exosystems in education identified in this book. SDT starts from the premise that people have three basic psychological needs: autonomy, competence and relatedness. The central finding in this theory is that the more the social context supports individuals' basic psychological needs, the more they will be intrinsically ${ }^{1}$ motivated and as a result, the better their performance and well-being. This theory has been applied in empirical research in many fields, related to learning both within and outside of formal education settings. For example, studies in how children are motivated to learn (Ryan and Deci, 2000a, p. 368; Taylor et al., 2014); how people are motivated to live healthily (Ryan et al., 2008), or lose weight, along with other studies into aspects of health ( $\mathrm{Ng}$ et al., 2012); how organizations may manage change programs (Stone et al., 2009), how employees are motivated at work (Gagné et al., 2010); or how to motivate regulatees to comply with regulation (Six, 2013).

Weibel (e.g. 2007) and colleagues (e.g. Six, 2018; Weibel and Six, 2013) have applied SDT to a better understanding of the relationship between trust and 
control in work relationships (teachers' mesosystem) and regulation (part of the exosystem). Accountability can be conceptualized as a form of control. Formal control is about setting standards, gathering information and making judgements; possibly followed by interventions (Weibel, 2007). It is usually defined from the perspective of the controller. This involves the same activities as accountability (for definition, see Chapter 1), but accountability is usually defined from the perspective of the account giver (Bovens, 2005). This combination of research makes SDT a relevant and appropriate theory to further unpack how the relationship between trust and accountability motivates teachers to be or become effective.

Currently, in many countries, educational accountability systems are experienced as being dominated by distrust of teachers and schools, suggesting a negative relationship between trust and accountability, sometimes even leading to worse outcomes (e.g. Hoecht, 2006; Lasky, 2005). This, however, is not inevitable, as I intend to show in this chapter.

This chapter argues that schools and education systems can motivate teachers to be(come) effective when accountability and trust reinforce each other, creating a work context that supports teachers' basic psychological needs. The first section addresses the different perspectives on the relationship between trust and accountability and introduces SDT as the theoretical basis for understanding when the relationship is positive or negative. SDT is a motivation theory and is the focus of Section 2, where the three basic psychological needs for self-determination are addressed, and how they relate to intrinsic/extrinsic motivation and the social context. In Sections 3 and 4, these theoretical building blocks are applied to trust-based accountability regimes in the mesosystem (i.e. school) and the regulatory dimension of the exosystem. The chapter concludes with a reflection on how this contributes to the quest that is central to this book.

\section{Relationship between trust and accountability}

The concept of accountability is related to the more general concept of control, since the objective of an accountability system is to control the accountor's behaviour so that the accountee's interests and preferences are met (Bovens, 2005). Governance can also be seen as a form of control, as it aims to have all actors work towards what the 'governors' aim to achieve. Who the governors are and how the aims are defined depends on the governance arrangement. The first subsection looks at what each governance mechanism's perspective is on the relationship between trust and control/accountability. Since in practice, no one ideal-type mechanism exists in isolation, we need to look from a different conceptual perspective to shed light. In the wider trust and control literature, two perspectives on the relationship between trust and control have emerged from empirical studies; these are presented in the second subsection. 


\section{Perspectives on relationship trust and accountability in governance mechanisms}

How do the concepts of trust and control (accountability) relate to the three governance mechanisms from Chapter 2? What are the relevant assumptions and perspectives of each ideal-type governance mechanism for these concepts (cf Bradach and Eccles, 1989; Meuleman, 2008)?

In hierarchy governance, the assumption is that relations are based on authority, driven by compliance to a set of rules and procedures that is determined beforehand. Trust is not explicitly addressed, but implicitly leaders are trusted to have the best judgement and are, therefore, the actors who formulate the rules. Subordinates are only trusted to apply the rules and follow procedures. The attitude towards problems is that they should be avoided where possible using the rules and procedures. The rules provide upfront clarity about the desired results aimed for and clarity about the processes. This creates a low need for ex post verification and audit.

In market governance, the assumption is that relations are based on a market exchange between two independent actors. There is independence because both buyer and supplier have plenty of alternatives exchange partners to choose from. The exchange is driven by market prices and contracts. Another assumption is that actors pursue self-interest maximization with guile (cf Williamson, 1993) so cannot be trusted to take into account the other actor's interests. In this model, the market is implicitly trusted to solve all problems and has the best judgement. The contracts provide high upfront clarity about results, but low clarity about process, since it is usually not included in contracts. The assumption about self-interest maximizing actors creates high principal-agent risks, and therefore a high need for ex post verification and audit.

In network governance, the assumption is that relations are social and driven by professional values and competencies; actors are mutually interdependent to achieve their goals. Trust is explicitly part of network governance (Bradach and Eccles, 1989). In networks, actors come together because of their interdependence and need to build trust to be able to 'access' the potential benefits of collaborating in the network. Each actor has a contribution to make. Because of the uncertainty about the exact nature of the collaboration in the network, upfront clarity about results is low, but shared intentions, and where possible shared goals, are important in the trust-building process. Upfront clarity about the process is medium high as certain agreements about how to work together are needed, including process agreement how to deal with the inevitable problems and conflicts is crucial for a successful long-term collaboration. In networks, the need for audit and verification afterwards is related to the degree that partners trust each other.

In reality, none of the three ideal types can be observed in practice in education systems. There is always some hierarchy present, yet given the task characteristics of teaching, as teachers are alone in their classrooms, it is impossible for school directors, governors or inspectors to ensure full compliance. Teachers are 
street-level bureaucrats: they not only implement the rules but also make policy as they use their discretion to be responsive to student needs while taking the rules into account (cf Lipsky, 1980). More importantly, each student is different and a teacher must be responsive to their particular needs in order to achieve high learning outcomes. No set of rules and procedures is capable of covering all possible situations. This renders leaders dependent on their teachers for them to achieve their goals. So in practice there is always some combination of hierarchy, as with subordinates dependent on leaders, and networks, due to mutual interdependence, present in education systems. Over the past four decades or so, market forces have been introduced in education in many countries, leading to performance-based financing of schools and sometimes even pay-for-performance for teachers. Accountability systems based on high-stakes tests are a clear example of such reforms (see earlier chapters).

We see both positive and negative relationships between trust and accountability in education systems around the world. Can these two perspectives be related to the three governance mechanisms? Not directly, given that in practice they never occur in isolation. We revisit this question later.

\section{Perspectives on trust and control (accountability)}

In the trust literature, two perspectives on the relationship between trust and control are distinguished (e.g. Das and Teng, 2001; Weibel, 2007; Weibel et al., 2016). Both perspectives are observed in practice, also in education (e.g. Avis, 2003; Blomeke and Klein, 2013; Ghamrawi, 2011).

The dominant perspective is that trust and control have a negative relationship, that they substitute one another. In this perspective, the more you trust, the less you may control; and controls are experienced as 'organized distrust' (e.g. Avis, 2003). Controls are then experienced as signs of distrust (Ghoshal and Moran, 1996), for example 'audit implies a culture of distrust' (Cooke, 2006, p. 976). Controls are also seen to increase relational detachments, increasing the distance between people, which often leads to an 'us versus them'-mentality between managers and subordinates, for example when new managerial controls were met 'with distrust by the staff' (Bolton, 2004, p. 322).

The second perspective, which is also observed in practice but not as widespread, is that trust and control have a positive relationship, that they strengthen one another (Long and Weibel, 2018; Six, 2018; Weibel, 2007; Weibel et al., 2016). In this positive relationship, controls provide employees with predictability (Zaheer et al., 1998), enable employees to perform their jobs competently (Gittell, 2001), protect employees against arbitrary decisions by universally applying general rules (Child and Möllering, 2003) and reduce the risks of working together (Langfred, 2004). Weibel et al. (2016) point to the importance of the implementation of controls: well-implemented controls facilitate employees' trust in the organizations, while poorly implemented controls - inconsistent or overly rigid implementation - undermine trust. 
Given that these two relationships both occur in practice, the rest of this chapter addresses the questions, what are the contingencies that will make the positive relationship between trust and accountability (systems) more likely? And what are the conditions that need to be met for trust-based accountability? Trust-based accountability is facilitated when the accountors are intrinsically motivated. SDT helps to identify which conditions support intrinsic motivations (Ryan and Deci, 2017), so the first question is what is SDT?

\section{Self-determination theory and intrinsic motivation}

According to SDT, human beings have three basic psychological needs: the need for autonomy, competence and relatedness. SDT builds on earlier theories of intrinsic versus extrinsic motivation. It addresses the question, under which contextual conditions do individuals motivate themselves (Deci and Flaste, 1995; Gagné and Deci, 2005; Ryan and Deci, 2000b). In essence, the answer SDT proposes is those social contexts that facilitate self-determination have a positive effect on intrinsic motivation. Self-determination is enhanced if the three basic psychological needs are satisfied (Deci and Ryan, 2000; Ryan and Deci, 2000b).

\section{Three basic psychological needs: Autonomy, competence and relatedness}

The need for autonomy is central to SDT. Individuals feel autonomous to the degree that they perceive their behaviour to be discretionary rather than imposed by others; in this sense, they experience a sense of choice and volition. Autonomy is not independence, one may feel autonomous while being (inter)dependent on others. De Charms (1968) argued that perceiving oneself (yourself) as the locus of causality for your own behaviour is the fundamental requirement for intrinsic motivation. The need for autonomy is supported the more individuals are given the opportunity 'to freely process and endorse transmitted values and regulations' and the opportunity to give 'personal meaning and valence to acquired regulations' (Deci and Ryan, 2000, p. 238). Autonomy is also supported when rewards and sanctions 'are administered on the basis of a broad and more subjective evaluation', rather than 'strictly contingent on the evaluation of standard fulfilment' (Weibel, 2007, p. 509). In other words, autonomy is supported when more narrative accountability is possible, rather than mere numbers without context (De Bruijn, 2007).

In SDT, the need for competence has two components: individuals may feel supported in their competence development when they perceive that they can develop new skills and mental frames. The first component may be traced back to White's (1959) concept of mastery and of Bandura's (1977) concept of selfefficacy. The second component, self-esteem, has been proposed to be a strong, pervasive motive to maintain a certain level of positive feelings about oneself 
(Jones, 1973). Competence is supported the more individuals can 'understand or grasp the meaning or rationale behind the regulation and an ability to enact it' (Deci and Ryan, 2000, p. 238). The more feedback that individuals receive that is constructive and learning oriented, the more this process is enhanced (Weibel, 2007).

People want to feel part of and accepted by the social groups they live in (e.g. Reis et al., 2000); their need for relatedness is about the desire to experience meaningful, supportive social relationships. The need for relatedness is supported when individuals are able to internalize the values and regulations of their social groups (Deci and Ryan, 2000, p. 238). Relatedness is also enhanced if the authorities within the group, such as school leaders, impose formal controls with positive intentions, in other words, if they trust rather than distrust those controlled (Weibel, 2007). Mutual understanding, trust and respect are important (Ryan and Deci, 2017).

\section{Self-determination and intrinsic (autonomous) motivation}

When performing self-determined activities, people have an internal perceived locus of causality. They are what SDT calls autonomously motivated. When, however, external rewards are introduced for these activities, 'people tend to feel controlled by the rewards, prompting a shift in the perceived locus of causality for the behavior from internal to external' (Deci and Ryan, 2000, p. 234). The motivation that people experience in the latter situations, SDT calls controlled motivation. It occurs not only when 'a person feels externally ... pressured or compelled to act', but also when 'the person is motivated by guilt, shame, contingent self-esteem, and fear of disapproval' (Ryan and Deci, 2017, p. 14); in other words, whenever people feel forced 'to act in ways that are incongruent or alien with respect to the person's sense of self' (Ryan and Deci, 2017, p. 14).

SDT posits that the more people internalize and integrate socially sanctioned values and norms, the more self-determined their actions and the more likely it is that their locus of causality is internal. This internalization process, however, does not occur automatically. 'The degree to which people are able to actively synthesize cultural demands, values, and regulations and to incorporate them into the self is in large part a function of the degree to which fulfillment of the basic psychological needs is supported as they engage in the relevant behaviors' (Deci and Ryan, 2000, p. 238). Social contexts that support people's basic psychological needs 'facilitate more self-determined and high-quality functioning' (Ryan and Deci, 2017, p. 12). In such contexts, people are autonomously motivated. Autonomous motivation occurs both when people do things "out of interest and for which the primary "reward" is the spontaneous feelings of effectance and enjoyment that accompany the behaviors', and when behaviours yield 'outcomes that are personally valued or important' (Ryan and Deci, 2017, p. 14). 
What SDT researchers call autonomous motivation, most people call intrinsic motivation. Hence this chapter will refer to autonomous motivation as intrinsic motivation. So the more self-determined people are, the more intrinsically (autonomously) motivated. This leads to many benefits to the people involved, their organizations and beyond (Ryan and Deci, 2017, p. 17).

More self-determined functioning is associated with greater creativity, superior learning, better performance, enhanced well-being and higher quality relationships.

And the argument in this book is that this outcome is also the desired outcome of accountability systems in education.

\section{Trust-based accountability: Trust, accountability and intrinsic motivation}

The core statement of SDT - regardless of which field it is applied to - is that the more the social context supports people's basic psychological needs, the more they will be intrinsically motivated and, as a result, the better their performance and well-being (Ryan and Deci, 2017). Figure 3.1 shows this core conceptual model.

Given the focus in this book on teachers as central actors for achieving high education quality, teachers need to be intrinsically motivated to be(come) effective. In terms of the systems introduced in Chapter 1, their mesosystem (the school) is their direct social context that needs to support their basic psychological needs. Research shows that the exosystem - outside the school - exerts such influence on school leaders that this also influences teachers' intrinsic motivation and thus students learning outcomes. Within the exosystem, the focus is on regulation since this is a major factor in the exosystem. The next two sections present research for each of these systems.

In SDT's terminology and applied to teachers, the more the accountability system - at both meso- and exo-level - supports teachers' basic psychological needs, the more they will be intrinsically motivated and, as a result, the better the learning outcomes. And as a result, the more school leaders will trust them, which is part of the relatedness need and thus further strengthens the supportive accountability (see Figure 3.2). In contrast, the more the accountability system thwarts teachers' basic psychological needs, the more intrinsic motivation will

\begin{tabular}{|c|c|c|c|c|}
\hline $\begin{array}{l}\text { Social context supports } \\
\text { psychological needs }\end{array}$ & + & $\begin{array}{l}\text { Intrinsic } \\
\text { motivation }\end{array}$ & + & $\begin{array}{l}\text { Better performance } \\
\text { Higher well-being }\end{array}$ \\
\hline
\end{tabular}

FIGURE 3.1 The core conceptual model of self-determination theory 


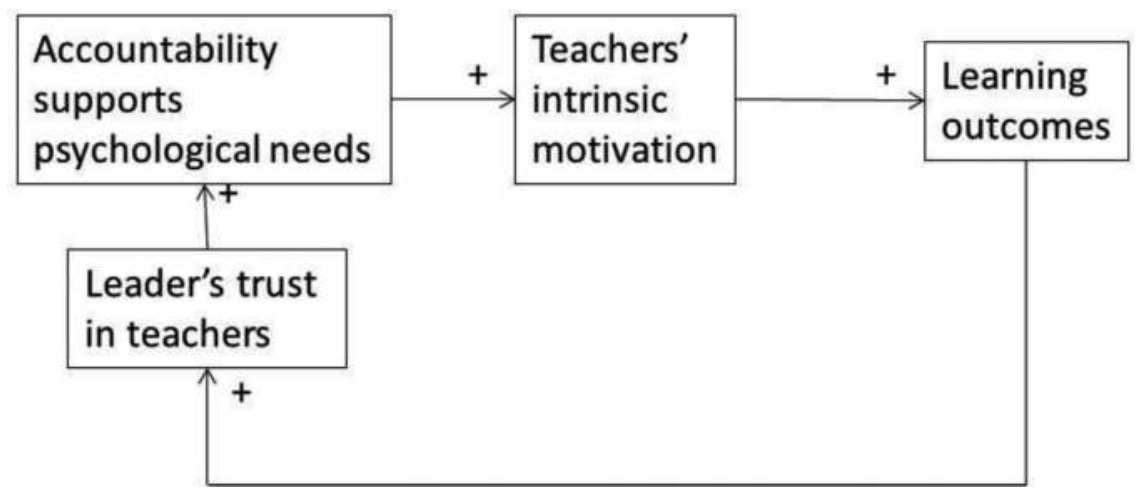

FIGURE 3.2 Trust, accountability and intrinsic motivation strengthening each other

be crowded out by extrinsic motivation and the lower the learning outcomes are likely to be. This will lead to lower trust by school leaders (and inspectors) and possibly even distrust, which further strengthens the accountability system that thwarts the basic needs.

It is important to note here that in this model, it is not a question of how much control or accountability leads to trust or distrust, but rather how the accountability measures are experienced by those who are accountable (i.e. the teachers). Does the accountability system enable and support them in what they are trying to achieve, does it motivate them; or does it hinder them, trying to coerce them (cf Adler and Borys, 1996)? Since this is a different approach to the three ideal-type governance mechanisms presented in Chapter 2, it cannot neatly be applied to these three governance mechanisms, we address this issue next.

\section{Trust and accountability in the mesosystem}

How can teachers be motivated to support students' learning processes? Examples of teacher behaviours that promote students' intrinsic motivation are listening to students, giving students ample time to independent work, giving students opportunity to talk, encouraging effort and acknowledging students' experiences and perspectives (Ryan and Deci, 2017, p. 368). Teachers, in turn, need a context - their work context - that supports their psychological needs. How can accountability systems that support teachers' needs and school leaders' trust in teachers strengthen each other in intrinsically motivating teachers? A recent literature review across different public sectors and public professionals into the relationship between trust in their professional autonomy and managerial control found two models (Six, 2018). The dominant model showed an adversarial relationship between professionals and their managers and is based on a negative relationship between trust and accountability. Professionals experienced their work context as controlling and as thwarting their psychological needs. This 
had a negative effect on outcomes and performance and triggered distrust in their managers, which reinforced the thwarting context creating a vicious cycle of increasing distrust and lower intrinsic motivation. The other model is more supportive and collaborative and is based on a positive relationship between trust and accountability. It is less frequently present than the dominant model. So it is possible in the public sector - and in education more specifically - to have trust and control/accountability strengthen each other.

What does this supportive context look like in education? In their review of decades of SDT research in education, Ryan and Deci (2017, p. 381) conclude that:

[...] education models and policies that aim to facilitate satisfaction of the basic psychological needs of students and teachers thus creating positive changes not only in students' and teachers' motivations and experiences, are most likely to optimize both achievement and broader developmental outcomes [italics added by author].

Especially high-stakes testing policies, which have been introduced in many school settings around the globe, have a broad range of negative effects on both students and teachers.

When teachers are pressured from above (e.g. with an accountability emphasis) or from below (e.g. from disengaged students), the teachers tend to become less supportive of students' autonomy and basic needs (Ryan and Deci, 2017, p. 380).

SDT has also been applied to work motivation more generally, and a review of that literature led to the list of conditions for a positive trust-accountability relationship shown in Figure 3.3.

\section{Need for autonomy}

The need for autonomy support is facilitated when professionals are able to participate in the development and execution of formal control or accountability measures (Weibel and Six, 2013). When professionals experience the support for their own initiatives and have choices and discretion, then this also supports autonomy (Hardré and Reeve, 2009). The participation in the development of accountability systems is not enough, the interpretation of the information that is generated and evaluated is also important as it allows for the perspective of professionals to be taken into account (De Bruijn, 2007).

Studies in education that do not explicitly use SDT showed the importance of a dialogue across a range of stakeholders so that different views could be heard and creative responses were found to deal with today's radical uncertainty (e.g. Avis, 2003). Teaching quality improved when teachers 


\section{Psychological needs Conditions}

\begin{tabular}{|c|c|}
\hline Autonomy & Participation in development/execution of formal controls \\
\hline & Support for own initiative \\
\hline & Opportunity for choice \\
\hline & $\begin{array}{l}\text { Dialogue about interpretation/implementation external } \\
\text { regulation }\end{array}$ \\
\hline Competence & Learning-oriented/constructive feedback \\
\hline & Opportunities for competence development \\
\hline Relatedness & Peer support \\
\hline & Manager trust in professionals \\
\hline & Equity and inclusion \\
\hline & Manager support: \\
\hline & - Managers take professionals' perspectives \\
\hline & - Managers understand professionals' feelings/ beliefs \\
\hline
\end{tabular}

FIGURE 3.3 Organizational conditions for positive trust-control relationship for professionals

Source: Ryan, R. M., \& Deci, E. L. (2017). Self-determination theory. In: Basic Psychological Needs in Motivation, Development and Wellness. New York: Guildford Press; Weibel, A. (2007). Formal control and trustworthiness: Shall the twain never meet? (pp. 57-81). Group \& Organization Management, 32(4), 500-517; Weibel, A., \& Six, F. E. (2013). Trust and control: The role of intrinsic motivation. In: R. Bachmann \& A. Zaheer (Eds.), Handbook of Advances in Trust Research. Cheltenham: Edward Elgar.

experienced more autonomy (Blomeke and Klein, 2013). More congruence between teachers' classroom values and the school system's values was built when teachers engaged in a professional dialogue with colleagues and school leaders (Ghamrawi, 2011).

\section{Need for competence}

The need for competence support is facilitated when feedback is given to professionals, that is learning oriented and constructive (Weibel and Six, 2013). This feedback may come from peers or managers. Constructive feedback from school leaders to teachers strengthens intrinsic motivation and has a positive effect on education quality (Ghamrawi, 2011; Nyhan, 2000). When teachers receive 
more frequent constructive appraisals, teaching quality improves (Blomeke and Klein, 2013).

The more professionals experience opportunities to develop their competences further, the more intrinsically motivated they are likely to be (Weibel and Six, 2013). Professional reflection is part of more general competence development and facilitates dealing with complex and ambiguous situations (Brown et al., 2011; Ruch, 2005).

\section{Need for relatedness}

For relatedness support, experiencing meaningful social relationships with peers and managers is key. Accountability systems that focus on intrinsic work engagement and manager trust facilitate professionals' intrinsic motivation (Weibel and Six, 2013). Both school leaders trust in teachers and teachers' trust in their school leader or school have positive effects on teachers intrinsic motivation (Blomeke and Klein, 2013; Ghamrawi, 2011). Trust among teachers has a positive effect on motivation, work engagement and teaching quality (Ghamrawi, 2011). Managers who get directly involved with front-line work better understand professionals' beliefs and preferences and help professionals experience manager support. It also reduces the need for audits, 'I can gain far more knowledge about the quality of a [hospital] ward by working on it than I could on a thousand audits' (Cooke, 2006, p. 980).

\section{Accountability in schools (mesosystem)}

So the more the mesosystem supports teachers' basic psychological needs and hence stimulates their intrinsic motivation, the better teachers are able to be or become effective. Studies, which investigated the impact of directly training teachers and their supervisors to be more supportive of students' and employees' psychological needs respectively, found that 'the more autonomy-supportive the supervisors and teachers are, the more positive will be the consequences for their employees and students' (Edmunds et al., 2008; Hardré and Reeve, 2009; Ryan and Deci, 2017, p. 379).

In their overview of SDT research in education, Ryan and Deci (2017, Chapter 14) found that several factors in schools interfere with teachers' basic psychological needs satisfaction, which in turn led these teachers to be more controlling and the students less self-determined. Accountability is one such factor. In recent decades, accountability demands in the exosystem in education have introduced more and more (high stakes) tests at different ages. High-stakes testing programmes put pressure on school leaders. School leaders will pass this pressure on to teachers by being more controlling and less needs supportive; and the teachers in response will be more controlling with students, undermining students' intrinsic motivation and learning (Deci et al., 1982). Students indicated that they preferred to be taught by teachers who were not burdened by accountability pressures. 
In sum, the more schools (the mesosystem) provide a work context for teachers that supports them in their psychological needs - autonomy, competence and relatedness, the more they will be intrinsically motivated to provide the learning context for students that support their needs for autonomy, competence and relatedness, which in turn will lead to better learning outcomes and higher well-being for students and teachers.

In terms of the different forms of governance, the tentative conclusion related to market governance may be that the introduction of market mechanisms such as high-stakes testing and pay-for-performance usually leads to a negative relationship between trust and accountability. What seems to meet the conditions for a positive relationship is a combination of hierarchy and network governance. The 'right' combination is achieved when teachers experience sufficient autonomy to influence the rules and procedures in the accountability system; and the hierarchical relations are experienced as meaningful social relations based on trust.

The next section addresses what this implies for the regulatory context for schools (the exosystem). This context needs to support schools in providing the supportive context for teachers.

\section{Trust and accountability in the exosystem: The regulatory relation}

For schools to be able to provide the supportive work context for teachers as described earlier, they in turn need a context that supports them. The context within which schools operate - the exosystem - is multidimensional: regulation, parents, pupils, local community and funders. This section looks at the regulatory context for schools. Schools as actors do not literally have basic psychological needs, but Six (2013) showed that the concepts of need for autonomy, competence or relatedness can be meaningfully applied in regulatory relationships to analyse what types of relationships lead to better compliance.

\section{The regulatory trust triangle}

Without regulation, every individual must collect the information necessary to make a good judgement about the trustworthiness of the organizations that they engage with, such, for example, as the schools they want to send their children to. This is not only inefficient but often impossible. Often the information requires expertise to analyse and interpret properly. Not every parent can properly judge the quality of education. This particular challenge that parents face is irrespective of whether their relationship with the school is governed by hierarchy, market or network.

It makes sense to have a party that has the required expertise to look at it and make the judgement. That is where the regulator comes in. It is more efficient 


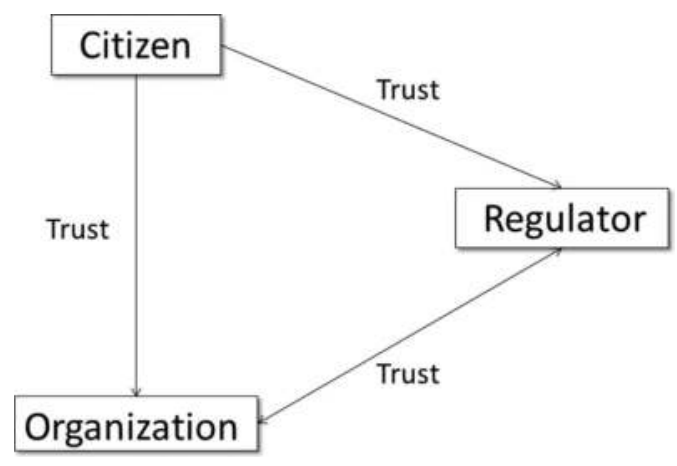

FIGURE 3.4 The regulatory trust triangle

Source: Six, F. E., \& Verhoest, K. (Eds.). (2017). Trust in Regulatory Regimes. Cheltenham: Edward Elgar.

if one independent and competent actor collects the required information and makes the judgement with the required expertise (Six and Verhoest, 2017). This is an example of third-party role trust (Nooteboom, 1999). It means that when an actor is not in the position to make a good judgement about the trustworthiness of another actor, but they trust a third actor who says the second actor is trustworthy, then the first actor is more likely to trust this second actor. So when a citizen is not in a position to properly judge whether they can trust the school to provide good quality education to their children; and when the regulator says 'I have had a good look and I trust that school'; and the citizen trusts the regulator, they will more likely trust the school (see Figure 3.4). Thus regulators help lubricate social relations with trust (Zucker, 1986).

This only works if there is trust in all three relations. Many inspectors, supervisors or auditors say that trust is not appropriate in the relationship between regulator and regulated organization. They have been told when they entered their profession that they should do their inspections and audits based on "healthy distrust'. That is appropriate, they say, because if everyone could be trusted, we would not be needed. ${ }^{2}$ So it goes to their identity. Looking at the triangle, if the regulator generally distrusts organizations they inspect, then the citizen, because they trust the regulator, will follow their judgement and therefore will also distrust organizations. So the positive impact of a regulator lubricating social relationships is lost. Of course, when the distrust is justified, when a school really is worthy of distrust because it does not perform and is failing, then the regulatory judgement of the school is very helpful to citizens. And the regulator should act on that distrust by maybe even closing the school.

In regulatory regimes, the provision of third-party trust by regulators is only useful as long as citizens trust regulators. "We trust that regulatory judgements are being made honestly by appropriately knowledgeable, motivated and qualified 
people, who are credibly representing the public for whom they act as proxies' (Downer, 2010, p. 94). When a regulator is distrusted by citizens, its judgement is irrelevant to them and therefore has no impact. People only take on board a judgement from a party that they trust. For example, when an inspectorate who is distrusted judges that a school is trustworthy, parents are likely to ignore that judgement due to their distrust of the inspector. If, on the other hand, the inspectorate is trusted, parents are likely to take that judgement into account in their decision process.

These three relations form the basic trust triangle for regulation between citizen, regulated organization and regulator, in our context: students and their parents, school and the education inspectorate. Next, the focus is on the regulatory relation between regulator (inspectorate) and regulated organization (school). What do we know about the role of trust in these regulatory relations?

\section{Trust in regulatory relations}

The most dominant theory in regulation research -responsive regulation theory (RRT, Ayres and Braithwaite, 1992) - places cooperation and trust at the heart. RRT recommends 'a dynamic regulatory strategy of dialogue and trust as a first choice followed by escalation to more punitive regulation when trust is abused' (Braithwaite and Makkai, 1994, p. 1).

Hence, trust within regulatory relations has received a fair amount of attention in research, especially with respect to its impact on compliance (e.g. Braithwaite and Makkai, 1994; Gunningham and Sinclair, 2009a; Heimer and Gazley, 2012; Murphy, 2004). These studies focus on the interaction between the individual inspector, representing the regulator, and the individuals, representing the regulated organization in the regulatory encounter (often the school director). They distinguish inspector trust in the regulatee from regulatee trust in the inspector and study how these two trust relations interact. Overall these studies suggest that regulator trust in regulatees and regulatee trust in the regulator have a positive effect on compliance, which in this book implies improving teaching quality.

Braithwaite and Makkai's (1994) longitudinal research shows that inspector trust has a positive effect on compliance. More precisely, they find that the more care home directors perceive that inspectors trust them, the better their compliance at the next inspection. Inspector trust in regulatees has another effect as well, since 'as long as [inspectors] believe a clinic is adhering to the spirit of the regulations, regulatory inspectors will join clinic staff in working around “inconsequential” technical mistakes' (Heimer and Gazley, 2012, p. 882). This does not necessarily imply regulatory capture, where a regulator loses its independence by being forced to act on behalf of the regulatees. After all, 'a good monitor knows when and how to join the performance and therefore how to get backstage to see what is really happening' (Heimer and Gazley, 2012, p. 882). 
Regulatee trust in inspectors is equally important and 'an opportunity to correct mistakes only occurs when there is trust on both sides-when regulators believe the clinic staff are competent and good-willed and they (the staff) are willing to acknowledge errors because they expect to be treated fairly' (Heimer and Gazley, 2012, p. 882). Procedural justice, fair treatment, is a key antecedent to regulatee trust in inspectors and an important driver of regulatees' intention to comply (Murphy, 2004, 2016; Murphy et al., 2009).

Six and Verhoest (2017) found four studies in different sectors and on different continents that studied all three parties in the regulatory trust triangle (Gouldson, 2004; Gunningham and Sinclair, 2009b; Holm and Halkier, 2009; Thiers, 2002). In these studies, the regulator's challenge of being the trusted third-party between citizens' interests on the one hand and the regulated business' interests on the other hand is highlighted. Citizen perceptions of the regulator are very sensitive to whether the regulator is seen as independent to business or as too cosy with business. In all four studies, regulators were seen by citizens, at least at some point in time, as being captured by the businesses they were supposed to inspect and as not paying enough attention to citizen concerns. So, the role of the regulator is ridden with dilemmas.

\section{Trust, regulation and intrinsic motivation}

The main argument in this chapter is that the quality of education is positively influenced when teachers are intrinsically motivated to provide students with a supportive learning context. This implies that inspectors need to stimulate the intrinsic motivation of teachers and their school leaders. Six (2013) applied SDT conditions to regulatory relations and found support in the regulation literature for the need for autonomy-, competence- and relatedness-support (see Figure 3.5).

The need for autonomy support is acknowledged in regulatory concepts such as (enforced) self-regulation (Ayres and Braithwaite, 1992; Campbell, 2007), management-based regulation (Coglianese and Lazer, 2003; Gunningham and Sinclair, 2009a), the importance of normative motivations (Winter and May, 2001) and procedural and restorative justice (Braithwaite, 2002; Macrory, 2006; Makkai and Braithwaite, 1996; Nielsen and Parker, 2009). The need for competence support is acknowledged in the importance of educating regulatees about rules, norms and how to comply (Ayres and Braithwaite, 1992), constructive feedback (Braithwaite et al., 2007) and praise (Makkai and Braithwaite, 1993). And last, the need for relatedness support is acknowledged by stressing the importance of trust in the regulatory relation (Braithwaite and Makkai, 1994; Murphy, 2004); the role of social motivations, norms and controls (Campbell, 2007; Winter and May, 2001); procedural justice (Murphy et al., 2009) and joint performances (Heimer and Gazley, 2012).

The more these conditions are present, the more regulatees are likely to be motivated to comply with the regulations and therefore, the more regulators 


\section{CONDITIONS THAT SUPPORT PSYCHOLOGICAL RELATED CONCEPTS IN REGULATION NEEDS

\section{Autonomy}

- Opportunity for regulatee to freely process and endorse transmitted values and regulation

- Opportunity to give 'personal' meaning and valence to acquired regulation

- Internal locus of causality

- Degree of participation

- Combination of rewards/evaluation

- Tailor-made regulatory arrangements
- Self-regulation (Campbell, 2007)

- Enforced self-regulation (Ayres \& Braithwaite, 1992)

- Self-regulation (Campbell, 2007)

- Enforced self-regulation (Ayres \& Braithwaite, 1992)

- Normative motivations (Winter \& May, 2001)

- Enforced self-regulation (Ayres \& Braithwaite, 1992)

- Management-based regulation (Coglianese \& Lazer, 2003; Gunningham \& Sinclair, 2009a; Parker, 2003)

- Restorative justice (Braithwaite, 2002; Macrory, 2006; Nielsen \& Parker, 2009)

- Procedural justice and control (Makkai \& Braithwaite, 1996)

- Regulatory arrangements (Braithwaite, 2008)

\section{Competence}

- Ability to understand or grasp meaning or rationale behind regulation

- Ability to enact regulation

- Constructive feedback
- Knowledge of rules (Winter \& May, 2001)

- Educating regulatees (Ayres \& Braithwaite, 1992)

- Speak and understand regulator language (Mascini \& van Wijk, 2009)

- Capacity to comply (Winter \& May, 2001)

- Constructive feedback (Braithwaite et al., 2007)

- Praise (Makkai \& Braithwaite, 1993)

\section{Relatedness}

- Internalize values and regulations of ones social groups

- Positive intentions regulator

- Perspective taking by regulator
- Social motivations, social norms (Winter \& May, 2001)

- Social control, normative calls (Campbell, 2007)

- Regulator trust in regulatee (Braithwaite \& Makkai, 1994; Murphy, 2004)

- Procedural justice (Murphy et al., 2009)

- Joint performances (Heimer \& Gazley, 2012)

FIGURE 3.5 Conditions that support psychological needs and regulation theory

Source: Six, F. E. (2013). Trust in regulatory relations: How new insights from trust research improve regulation theory. Public Management Review, 15(2), 163-185; Six, F. E., \& Verhoest, K. (Eds.) (2017). Trust in Regulatory Regimes. Cheltenham: Edward Elgar. 
are able to trust them, which further stimulated relatedness and motivation to comply (Six, 2013).

\section{Trust-based accountability in education}

What does all of the above imply for the possibility for trust-based accountability regimes in education? The more the regulator controls (rules and regulation) in the exosystem support the basic psychological needs of the regulatees, the more the regulatees will internalize regulator values; and the higher regulatee compliance will be. So the more the inspectorate does their inspection in such a way as to support the psychological needs of the schools and the teachers within them, the more teachers will internalize the regulator values, because there will be more of a match, and, as a result, better compliance. The higher the compliance, the more the regulators can trust. And trust being part of relatedness directly impacts on the support for psychological needs and internalization of values.

So what does this imply for regulators? First of all, procedural justice is very important. No matter how poorly the school performs, they should always treat teachers and school leaders with high procedural justice so that teachers and school leaders feel they are treated fairly. Second, they should be aware of their own general propensity to trust as an inspector, because it has an impact. If inspectors perform their inspection or audit from an attitude of distrust, it will be noticed by the regulatees, they will sense it. Regulatees will, in response, more likely be not as forthcoming and open with information as they otherwise might have been. The best way to show one's trustworthiness is often to start trusting in the first place. To be able to do this well, good interpersonal, communicative and reflexive competencies are needed. When taking the leap of faith required in trust, you then need to stay alert, stay inquisitive and observant of what happens. And reflect on whether your trust is still justified. In sum, trust is hard work and sometimes high risk, and it is often easier to work from distrust in regulatory relations.

In terms of what this implies for the three ideal-type governance mechanisms: state regulation is, by definition, hierarchical based on the state's authority to inspect and enforce regulation. The inevitable and long-term nature of the regulatory relationship also makes it a mutually dependent relationship, with network-like characteristics. The motivation to comply with regulation is strengthened when regulatees - teachers and school leaders - experience autonomy in the sense that they experience that they have had influence on the design and execution of the regulations. And when they experience meaningful social relationships with the inspectors who inspect them and that these act with high procedural justice. And research so far predominantly shows that the introduction of market mechanisms such as high-stakes testing usually leads to a negative relationship between trust and accountability, as shown previously. 


\section{Conclusion}

This chapter illustrates that the relationship between trust and accountability (control) can be either positive or negative. Using SDT, it argues that a positive relationship will more likely lead to better education quality than a negative relationship. This has implications at all system levels, and this chapter focused on two: the mesosystem (the school) and the regulator's influence in the exosystem.

In general, SDT talks about the social context within which each actor operates, which consists of more actors than have been included in the analysis. For example, the context for schools and teachers (the exosystem) consists of more than the regulator; it also includes parents, local authorities and, in some cases, private owners. Regulators have more formal authority over schools than most other actors, hence the focus on them. If other actors want to achieve high education quality, they should all want to focus on providing a social context for teachers (in their mesosystem) and schools (in the exosystem) that supports them in their psychological needs. This may be easier said than done, since the values and norms of what good education entails are contested (in the macrosystem).

The three central concepts in this book are trust, accountability and capacity. This chapter only looked in more detail at the relationship between the first two. Capacity consists of two dimensions: individual teacher's competencies and the resources available in the system, in particular within the schools. One of the basic psychological needs is to be acknowledged in the competencies one has as a teacher and to be supported in the desire to further develop one's competencies as a teacher, which relates to the first dimension of capacity. Regarding the second dimension, public services always operate with a shortage of resources, since demand always outstrips available resources (cf Lipsky, 1980) and public education is no exception. When resources become too constrained, stricter rules are often imposed which is likely to lead to experiences of lack of autonomy support. Lack of funds and time for skills development and training leads to lack of

competence support. And lack of resources also triggers coping mechanisms (cf Lipsky, 1980) that are likely to lead to less interest in the perspective of others and more self-preservation, which are likely to be experienced as lack of relatedness support. A continued focus on support for their psychological needs even when resources are constrained, however, is likely to enable teachers in their effort to be or become effective.

In a way, SDT propagates an accountability regime in education where teachers are in the lead with support from school leaders and actors in the other systems. Evers and Kneyber's (2015) radical proposal to Flip the System in education with high collective autonomy for teams of teachers, - note not individual autonomy - is in line with SDT-supportive contexts. This is a combination of hierarchy and network governance, but with the traditional hierarchy being flipped; the teachers become the authority. It explicitly lacks market 
governance. Since their publication, several country-specific versions have been published, such as in Australia (Andrews et al., 2018). In the Netherlands, a non-profit initiative Stichting LeerKRACHT has developed lean-based methods to realize more collective autonomy for teacher teams in some 750 schools, ${ }^{3}$ an approach that focuses on network governance, with some hierarchy and as little market as possible. The impact of this way of working is being evaluated at the time of writing, but participating teachers, students and school leaders are predominantly enthusiastic and first indications suggest positive impact (Stol and Stokking, 2014).

In sum, schools and education systems can motivate teachers to be(come) effective when accountability and trust reinforce each other, creating a work context that supports teachers' basic psychological needs. A challenge very worthwhile pursuing!

\section{Notes}

1 SDT uses the term autonomous motivation for what most people call intrinsic motivation. To stay close to everyday language, this chapter uses the term intrinsic motivation where formally it should use autonomous motivation.

2 Personal communication to the author by many inspectors, accountants or auditors during and after she gave presentations about this topic between 2009 and 2018 (time of writing).

3 In 2018.

\section{References}

Adler, P. S., \& Borys, B. (1996). Two types of bureaucracy: Enabling and coercive. Administrative Science Quarterly, 41(1), 61-89.

Andrews, J., Paterson, C., \& Netolicky, D. M. (2018). Flip the System Australia:What Matters in Education. London: Routledge.

Avis, J. (2003). Re-thinking trust in a performative culture: The case of education. Journal of Education Policy, 18(3), 315-332.

Ayres, I., \& Braithwaite, J. (1992). Responsive Regulation, Transcending the Deregulation Debate. New York: Oxford University Press.

Bandura, A. (1977). Self-efficacy:Toward a unifying theory of behavioral change. Psychological Review, 84(2), 191-215.

Blomeke, S., \& Klein, P. (2013). When is a school environment perceived as supportive by beginning mathematics teachers? Effects of leadership, trust, autonomy and appraisal on teaching quality. International Journal of Science and Mathematics Education, 11(4), 1029-1048.

Bolton, S. C. (2004). A simple matter of control? NHS hospital nurses and new management. Journal of Management Studies, 41(2), 317-333.

Bovens, M. (2005). Public accountability. In E. Ferlie, L. E. Lynn, \& C. Pollitt (Eds.), The Oxford handbook of public management (pp. 182-208). Oxford: Oxford University Press.

Bradach, J. L., \& Eccles, R. G. (1989). Price, authority and trust: From ideal types to plural forms. Annual Review of Sociology, 15, 97-118. 
Braithwaite, J. (2002). Restorative Justice and Responsive Regulation. Oxford: Oxford University Press.

Braithwaite, J., \& Makkai,T. (1994). Trust and compliance. Policing and Society, 4, 1-12.

Braithwaite, J., Makkai, T., \& Braithwaite, V. (2007). Regulating Aged Care, Ritualism and the New Pyramid. Cheltenham: Edward Elgar.

Brown, P., Alaszewski, A., Pilgrim, D., \& Calnan, M. (2011). The quality of interaction between managers and clinicians: A question of trust. Public Money \& Management, 31(1), $43-50$.

Campbell, J. L. (2007). Why would corporations behave in socially responsible ways? An institutional theory of corporate social responsibility. Academy of Management Review, 32(3), 946-967.

Child, J., \& Möllering, G. (2003). Contextual confidence and active trust development in the Chinese business environment. Organization Science, 14, 69-80.

Coglianese, C., \& Lazer, D. (2003). Management-based regulation: Prescribing private management to achieve public goals. Law \& Society Review, 37(4), 691-730.

Cooke, H. (2006). The surveillance of nursing standards: An organisational case study. International Journal of Nursing Studies, 43(8), 975-984.

Das, T. K., \& Teng, B. (2001). Trust, control and risk in strategic alliances: An integrated framework. Organization Studies, 22(2), 251-284.

De Bruijn, H. (2007). Managing Performance in the Public Sector. London: Routledge.

De Charms, R. (1968). Personal Causation: The Internal Affective Determinants of Behavior. New York: Academic Press.

Deci, E. L., \& Flaste, R. (1995). Why we do, what we do. In: Understanding Self-Motivation. Rochester: Penguin Books.

Deci, E. L., \& Ryan, R. M. (2000). The "what" and "why" of goal pursuits: Human needs and the self-determination of behavior. Psychological Inquiry, 11(4), 227-268.

Deci, E. L., Spiegel, N. H., Ryan, R. M., Koestner, R., \& Kauffman, M. (1982). Effects of performance standards on teaching styles: Behavior of controlling teachers. Journal of Educational Psychology, 74(6), 852-859.

Downer, J. (2010). Trust and technology:The social foundations of aviation regulation. British Journal of Sociology, 61(1), 83-106.

Edmunds, J., Ntoumanis, N., \& Duda, J. L. (2008). Testing a self-determination theory-based teaching style intervention in the exercise domain. European Journal of Social Psychology, $38(2), 375-388$.

Evers, J., \& Kneyber, R. (2015). Flip the System: Changing Education from The Ground. New York: Routledge.

Gagné, M., \& Deci, E. L. (2005). Self-determination theory and work motivation. Journal of Organizational Behavior, 26(4), 331-362.

Gagné, M., Forest, J., Gilbert, M.-H., Aubé, C., Morin, E., \& Malorni, A. (2010). The motivation at work scale:Validation evidence in two languages. Educational and Psychological Measurement, 70(4), 628-646.

Ghamrawi, N. (2011). Trust me: Your school can be better - A message from teachers to principals. Educational Management Administration \& Leadership, 39(3), 333-348.

Ghoshal, S., \& Moran, P. (1996). Bad for practice, a critique of the transaction cost theory. Academy of Management Review, 21(1), 1120-1171.

Gittell, J. H. (2001). Supervisory span, relational coordination and flight departure performance: A reassessment of postbureaucracy theory. Organization Science, 12(4), $468-483$. 
Gouldson, A. (2004). Cooperation and the capacity for control: Regulatory styles and the evolving influence of environmental regulations in the UK. Environment and Planning C: Government and Policy, 22(4), 583-603.

Gunningham, N., \& Sinclair, D. (2009a). Organizational trust and the limits of management-based regulation. Law \& Society Review, 43(4), 865-899.

Gunningham, N., \& Sinclair, D. (2009b). Regulation and the role of trust: Reflections from the mining industry. Journal of Law and Society, 36(2), 167-194.

Hardré, P. L., \& Reeve, J. (2009). Training corporate managers to adopt a more autonomy-supportive motivating style toward employees: An intervention study. International Journal of Training and Development, 13(3), 165-184.

Heimer, C. A., \& Gazley, J. L. (2012). Performing regulation: Transcending regulatory ritualism in HIV clinics. Law \& Society Review, 46(4), 853-887.

Hoecht, A. (2006). Quality assurance in UK higher education: Issues of trust, control, professional autonomy and accountability. Higher Education, 51(4), 541-563.

Holm, L., \& Halkier, B. (2009). EU food safety policy: Localising contested governance. European Societies, 11(4), 473-493.

Jones, S. (1973). Self- and interpersonal evaluations -esteem theories versus consistency theories. Psychological bulletin, 79, 185-199.

Langfred, C. W. (2004). Too much of a good thing? Negative effects of high trust and individual autonomy in self-managing teams. Academy of Management Journal, 47(3), 385-399.

Lasky, S. (2005). A sociocultural approach to understanding teacher identity, agency and professional vulnerability in a context of secondary school reform. Teaching and Teacher Education, 21(8), 899-916.

Lipsky, M. (1980). Street-Level Bureaucracy, Dilemmas of the Individual in Public Services. New York: Russell Sage Foundation.

Long, C. P., \& Weibel, A. (2018). Two sides of an important coin: Outlining the general parameters of trust-control research (pp. 506-521). In: R. H. Searle, A.-M. Nienaber, \& S. B. Sitkin (Eds.), The Routledge Companion to Trust. London: Routledge.

Macrory, M. (2006). Restorative Justice, Making Sanctions Effective. Retrieved from London.

Makkai, T., \& Braithwaite, J. (1993). Praise, pride and corporate compliance. International Journal of Sociology of Law, 21, 73-91.

Makkai, T., \& Braithwaite, J. (1996). Procedural justice and regulatory compliance. Law and Human Behavior, 20(1), 83-98.

Meuleman, L. (2008). Public Management and the Metagovernance of Hierarchies, Networks and Markets: The Feasibility of Designing and Managing Governance Style Combinations. Rotterdam: Springer.

Murphy, K. (2004). The role of trust in nurturing compliance: A study of accused tax avoiders. Law and Human Behavior, 28(2), 187-209.

Murphy, K. (2016).Turning defiance into compliance with procedural justice: Understanding reactions to regulatory encounters through motivational posturing. Regulation $\mathcal{E}$ Governance, 10(1), 93-109.

Murphy, K., Tyler,T. R., \& Curtis, A. (2009). Nurturing regulatory compliance: Is procedural justice effective when people question the legitimacy of the law? Regulation \& Governance, $3(1), 1-26$.

Ng, J.Y.Y., Ntoumanis, N., Thøgersen-Ntoumani, C., Deci, E. L., Ryan, R. M., Duda, J. L., \& Williams, G. C. (2012). Self-determination theory applied to health contexts: A metaanalysis. Perspectives on Psychological Science, 7(4), 325-340.

Nielsen, V. L., \& Parker, C. (2009). Testing responsive regulation in regulatory enforcement. Regulation \& Governance, 3, 376-399. 
Nooteboom, B. (1999). The triangle: Roles of the go-between (pp. 341-355). In R. T. A. J. Leenders \& S. M. G. (Eds.), Corporate Social Capital and Liability. Boston: Kluwer Academic Publishers.

Nyhan, R. C. (2000). Changing the paradigm: Trust and its role in public sector organizations. The American Review of Public Administration, 30(1), 87-109.

Reis, H. T., Sheldon, K. M., Gable, S. L., Roscoe, J \& Ryan, R. M. (2000). Daily well-being: The role of autonomy, competence and relatedness. Personality and Social Psychology Bulletin, 26(4), 419-435.

Ruch, G. (2005). Relationship-based practice and reflective practice: Holistic approaches to contemporary child care social work. Child and Family Social Work, 10, 111-123.

Ryan, R. M., \& Deci, E. L. (2000a). Intrinsic and extrinsic motivations: Classic definitions and new directions. Contemporary Educational Psychology, 25(1), 54-67.

Ryan, R. M., \& Deci, E. L. (2000b). Self-determination theory and the facilitation of intrinsic motivation, social development, and well-being. American Psychologist, 55(1), 68-78.

Ryan, R. M., \& Deci, E. L. (2017). Self-determination theory. In: Basic Psychological Needs in Motivation, Development and Wellness. New York: Guildford Press.

Ryan, R. M., Patrick, H., Deci, E. L., \& Williams, G. C. (2008). Facilitating health behaviour change and its maintenance: Interventions based on self-determination theory. The European Health Psychologist, 10, 2-5.

Six, F. E. (2013). Trust in regulatory relations: How new insights from trust research improve regulation theory. Public Management Review, 15(2), 163-185.

Six, F. E. (2018). Trust in public professionals and their professions (pp. 361-375). In: R. Searle,A.-M. Nienaber, \& S. B. Sitkin (Eds.), Routledge Compendium to Trust. New York: Routledge.

Six, F. E., \&Verhoest, K. (Eds.) (2017). Trust in Regulatory Regimes. Cheltenham: Edward Elgar.

Stol, Y., \& Stokking, K. (2014). Onderzoeksrapport evaluatieonderzoek veranderaanpak leerKRACHT2013-2014. Retrieved from Utrecht.

Stone, D., Deci, E. L., \& Ryan, R. M. (2009). Beyond talk: Creating autonomous motivation through self-determination theory. Journal of General Management, 34, 75-91.

Taylor, G., Jungert, T., Mageau, G. A., Schattke, K., Dedic, H., Rosenfield, S., \& Koestner, R. (2014). A self-determination theory approach to predicting school achievement over time: The unique role of intrinsic motivation. Contemporary Educational Psychology, 39(4), $342-358$.

Thiers, P. (2002). From grassroots movement to state-coordinated market strategy: The transformation of organic agriculture in China. Environment and Planning C: Government and Policy, 20(3), 357-373.

Weibel, A. (2007). Formal control and trustworthiness: Shall the twain never meet? Group \& Organization Management, 32(4), 500-517.

Weibel, A., Den Hartog, D. N., Gillespie, N., Searle, R., Six, F. E., \& Skinner, D. (2016). How do controls impact employee trust in the employer? Human Resource Management, 55(3), 437-462.

Weibel, A., \& Six, F. E. (2013). Trust and control: The role of intrinsic motivation (pp. 57-81). In: R. Bachmann \& A. Zaheer (Eds.), Handbook of Advances in Trust Research. Cheltenham: Edward Elgar.

White, R. W. (1959). Motivation reconsidered: The concept of competence. Psychological Review, 66(5), 297-333.

Williamson, O. E. (1993). Calculativeness, trust and economic organization. Journal of Law and Economics, 36(1, part 2), 453-486. 
Winter, S., \& May, P. J. (2001). Motivation for compliance with environmental regulations. Journal of Policy Analysis and Management, 20(4), 675-698.

Zaheer, A., McEvily, B., \& Perrone, V. (1998). Does trust matter? Exploring the effects of interorganizational and interpersonal trust on performance. Organization Science, 9(2), 141-159.

Zucker, L. G. (1986). Production of trust: Institutional sources of economic structure, 1840-1920. Research in Organizational Behavior, 8, 53-111. 


\title{
4
}

\section{DISTRUSTING CONTEXTS AND CULTURES AND CAPACITY FOR SYSTEM-LEVEL IMPROVEMENT}

\author{
Jacqueline Baxter
}

\section{Introduction}

Critical debates on what constitutes good governance and its requirements have dominated public-sector management reforms across the developed world for the last 30 years. Part of the reason for these debates is that within that time there has been a unilateral shift away from bureaucratic linear forms of governance in public services, to networked forms of governance (Flinders, 2008). This shift has also been accompanied by the rise of new public management (NPM) forms of control as a result of the collapse of high economic growth rates and the alarming and unexpected 'stagflation': a stagnation of the economy combined with unemployment and inflation (Ferlie and Ongaro, 2015). The shift to networked forms of governance has also been marked by an upturn in public-private partnerships within the public services. However, despite the move away from linear steering of public services, the general tenets of what constitutes good governance have not changed and continue to include: participatory decision-making, accountability, transparency, efficiency, responsiveness and inclusiveness, respecting the rule of law and minimising opportunities for corruption. Transparency involves clear and public disclosure of information, rules, plans, processes and actions by governments, companies, organisations and individuals (Holmes, 1993). It is the principle that public affairs need to be conducted in the open and the concomitant public trust that this engenders. These factors are known to create a climate of trust within governance, whether this be at the national level, or in relation to particular public services (Braithwaite and Levi, 2003).

Transparent accountability is a key component within the governance of the public services (Ferlie and Ongaro, 2015), contributing to its capacity to improve. Accountability, or the willingness to accept responsibility or to 
account for one's actions, is a key element of a system's capacity to improve (Anderson, 2014). Anderson et al. identify three types of accountability: the first - compliance-orientated system, individuals are held accountable by rules and bureaucratic procedures; the second - professional accountability, is based around professional norms and ethics of professionals; the third - performance-based accountability, relies far more on management by objective/NPM techniques to hold individuals and organisations accountable by the results that they produce - in education, this is characterised by league tables and other performance measures (Anderson, 2005). Professionals working in the public services often find themselves responding to all three measures, depending upon the culture and context: for example in the particular case of the English public services, they have, for the last 30 years, been subject to NPM techniques that rely heavily on performance-based accountability, due to successive governments' belief that this will aid capacity to improve (Newman and Clarke, 2009). However, research since then has countered this belief, illustrating that targets often induce gaming behaviours, such as, in education, schools excluding poorly performing pupils (off rolling), in the belief that they will negatively impact results, thus lowering the school's position and reputation in the league tables - public statistics on school performance (Ozga and Segerholm, 2014).

In education, networked governance and the rise of NPM techniques have exerted considerable influence on education systems. Traditional bureaucratic forms of governance have been replaced by networked governance, and a rise in the role of supranational, multilateral agencies, such as the OECD, the World Bank and UNESCO, all of which, place policy pressures upon national schooling systems. In addition, the rise of marketisation and NPM, have, in many systems, such as England, created hybrid systems of education in which state funded education is governed and controlled by a number of nonstate actors (Burns and Koster, 2016). International comparators, statistical in nature, such as the OECD Pisa programme, have become central of governance for national governments. For example, in England, schools are judged according to league tables and their performance is measured against international school statistics in subjects such as English and maths (Ozga et al., 2013). Inspectorates of education have, in a number of countries, grown in significance in terms of measuring whether schools are successful or the converse. This combination of marketisation, NPM techniques and networked governance has, over time, heralded profound changes to systems, creating spaces for what Ball (2007) terms edu-business - the incursion of business interests in state education.

Good governance is both a condition for and outcome of good governance: good governance and accountability are predicated on a certain degree of trust: trust that the measures used to hold institutions and individuals accountable, are fair and just, and trust that these institutions and individuals will comply with the measures. For example if schools trust that school inspectors will be fair in 
their appraisal of schools, then they are much more likely to comply with suggestions for improvement by that inspectorate. Trust in government requires that institutions that hold individuals and organisations to account on behalf of government are themselves transparent and free of corruption; they will only attain the level of legitimacy necessary to hold actors and institutions accountable when upholding the highest standards of openness and moral conduct. In countries in which corruption is rife, and leaders model corrupt practices, it is highly likely that citizens will not trust state-run institutions - assuming that they too will be infused with corruption.

Good governance and accountability not only require trust, they also promote trust, particularly in establishing generalised trust - an abstract trust attitude that is directed towards people in general, including strangers. Trustworthiness of the state is the most important condition for such generalised trust as it generates interpersonal trust and the amount of social and economic capital in a society. This in turn affects the state's capacity to govern: the state's capacity to govern concomitantly has an impact on the legitimacy of the state's governing tools - organisations established to carry out governance processes, such as school inspection. The state's ability to govern also depends upon 'credible commitments, reputational effects, and self-enforcing institutions' that significantly reduce the citizen's need to expend effort in monitoring government. In the case of education, this includes local, as well as national, governance tools, such as local education departments or inspectorates. If these institutions are trusted to raise standards in schools, then citizens need to expend less effort in holding government to account for poor standards in schools. Rules and norms have a key role in establishing trust in the state along with bureaucratic arrangements that reward competence and relative honesty by bureaucratic agents (Braithwaite and Levi, 2003). Norms of behaviour, for example paying taxes, are rewarded in many Western democracies by the availability of a good standard of public services. In corrupt states in which taxes are channelled into areas that do not benefit the taxpayer, there is little incentive to pay tax. In systems in which tax avoidance is commonplace, this then becomes the behavioural norm discouraging those citizens who would have paid tax to think twice about doing so. Within this transaction, there needs to be a certain amount of trust: trust by the citizen but taxes will be used for the public good, and trust by the government that taxes will be paid.

These examples indicate how trust acts as lubricant for any public system to function, and it can have a positive reinforcing effect in building capacity for improvement. For example, this two-way trust scenario means that governments can plan ahead for improvements that will benefit citizens, and on the other hand, citizens trust that their money will be used to generate improvement to public services both now and in the future. This makes it much more likely that improvement will make both trust and expectation of improvement become the norm. The effect also operates on an interpersonal level as a high level of trust signifies a readiness of citizens to cooperate with each other and to work together 
to achieve improvements in distinguished from the general trust which arises, according to Rothstein (2011), from the institutional environment of laws, norms and standards on which people and organisations can rely. This creates what is known as 'social capital' - networks of relationship amongst actors, which enable and foster co-working. For example, in education, capacity is improved by high levels of social capital born of trust, in which teachers work together to improve teaching, or heads trusting their teachers to work well and implementing systems of CPD (continuing professional development), in order for teachers to develop further and to retain job satisfaction.

In the literature, trust/trusting is generally accepted to be an organising principal - a core assumption via which societies or individuals can be classified. Distrust is conceptualised as a separate element and is distinct from trust. Rather than positioning distrust as the opposite of trust, or a lack of trust, distrust is viewed as a constraining element: one which leads to negative perceptions of others' behaviour and limiting successful organisational outcomes (McEvily et al., 2003). A lack of trust in an organisation, for example, does not mean this is a distrusting organisation, merely that there is not a great deal of trust within it. However, an organisation which is categorically distrusting is one in which relationships and interactions are coloured and conditioned by distrust and negative perceptions of actors' behaviour. This way of conceptualising distrust is helpful in identifying and alleviating distrusting cultures, which exert inimical effects on a system. For example, in a school with high levels of distrust as part of its culture, teachers are unlikely to observe each other's teaching, with a view to offering critically constructive feedback, due to a feeling that they might be reported by the observer, for poor teaching methods, and that there may be repercussions because of that. On the other hand, in a school which manifests high levels of trust, research indicates that staff are more open to critique (constructive) and have confidence that peer observation leads to improvement (Bell and Mladenovic, 2008).

Such distrusting cultures, once established, are difficult to change and often impact negatively on systems as people are suspicious and doubt others' intentions, even when the intentions of actors within such systems are, in fact, honourable (Papakostas, 2016). Distrust affects the capacity of education systems to improve as it undermines social capital that favours continuous professional development (Scheerens, 2000).

Evidence suggests that in spite of considerable financial resources, certain education systems fail to improve. This chapter aims to understand the lack of improvement in education and examine how capacity to improve student learning outcomes is undermined by distrust and corruption and what key areas need to be addressed in order to overcome this. The chapter begins with a discussion of what is understood as capacity and how it can improve learning outcomes, it then moves to examine how distrust and corruption affect capacity. It then concludes with an exploration of how trust can be restored through moral leadership, role models, boundary spanners and accountability and transparency. 


\section{What is capacity and how can it improve learning outcomes}

The OECD (2011, p. 7) defines capacity as 'the ability of people, organisations and society a whole to manage their affairs successfully'. It is often spoken of in relation to social and cultural capital within a system or society, and is recognised in relation to the resources, 'hard and soft', that a system possesses, in order to be successful. It reflects the resource-based view of strategic management, in that planning for improvement needs to take careful account of the resources, both human and material, that the system or institution has at its disposal (Raffo et al., 2016). A commonly accepted cause of the creation of a distrusting culture and concomitant diminution of its capacity to improve, is corruption (Rothstein and Varraich, 2017).

Burns and Koster (2016, p. 26) outline capacity as existing on four principal levels:

1. Individual level: the means by which to support individuals (parents, teachers, headmasters and policymakers) as they face the demands of new developments in the local context by building on existing knowledge (human resources and knowledge management).

2. Institutional level: support of existing institutions (e.g. schools, district offices) in forming policies, effective organisational structures and good management (this includes building learning organisations).

3. System level: supporting system-level actors (e.g. policymakers, teacher unions) to be able to fulfil their roles in designing/implementing/evaluating educational policies.

4. Societal level: ensuring a supportive context in which individuals and organisations operate and interact with the external environment. This is linked to cultural capital in terms of the degree to which societies are able to tolerate the uncertainty associated with trusting behaviours.

Educational effectiveness research provides an understanding of the types of technical and human capital that need to be in place to improve student outcomes. Scheerens (2014) summarises results of review studies that were carried out in the 1990s (Cotton, 1995), and more recent review studies by David et al. (2000) and Muijs, (2014). According to Scheerens, there is clear consensus about the following main conditions of effective schooling and teaching over time:

- Achievement orientation and high expectations: a productive school climate, a school mission focused on achievement, shared vision and goals, high expectations that all students can achieve

- Cooperative atmosphere and an orderly climate: cooperative planning, a learning-oriented atmosphere consensus, orderly climate

- Clear goals on basic skills: focus on student learning, concentration on teaching 
- Frequent evaluation: appropriate monitoring, evaluative potential of the school, assessment

- Professional development: staff development, in-service training, a learning organisation

- Parental involvement: parent support, home-school partnership

- Strong leadership: educational leadership, school management and organisation, improvement-oriented leadership

- Effective instructional arrangements: classroom management, time on task, structured teaching, opportunity to learn, coordination in curriculum and instruction

The context of these studies is important: the above-mentioned studies were set in industrialised countries but reviews on school effectiveness research in developing countries suggest that resource input factors play a bigger role in explaining school effectiveness (Scheerens, 2000). Particularly, teacherpupil ratio, teachers' education, teachers' salaries, per pupil expenditure and availability of textbooks which all appear to have a positive effect on student outcomes.

The elements highlighted earlier do not exist in a vacuum, but form part of the wider system and context of education. Although at first glance they appear to be separate, they are in fact intrinsically interlinked via complex networks of material and social capital networks of relationships among people who live and work in a particular society.

The next part of the chapter explains how trust and distrust affect system capacity to improve, examining key elements that have been identified as contributing to trust and the converse.

\section{Ways in which trust enhances capacity, and distrust undermines capacity in education}

Trust, as outlined in earlier chapters, operates on both the interpersonal/micro and general/system level, each of which has particular impact on capacity. Levels of trust are outlined in Figure 4.1. As the diagram describes, micro-level perspectives on trust are mirrored at both institutional level and system level. In other words, the actions of actors at the micro level impact perceptions and trust in institutions, and trust in the system (Oomsels and Bouckaert, 2017b).

The diagram outlines three areas which are important in creating trust within an environment: integrity - the expectation that system actors will act in a just and fair manner; benevolence - the confidence that actors possess, that a system is fair and free of corruption; competence - that actors are confident that a system, for example education, will work in an effective manner, i.e. that it will provide a good education for learners.

Trust in an actor, institution or system engenders a certain amount of vulnerability on the part of the trustor, and it also involves a certain amount of risk. 


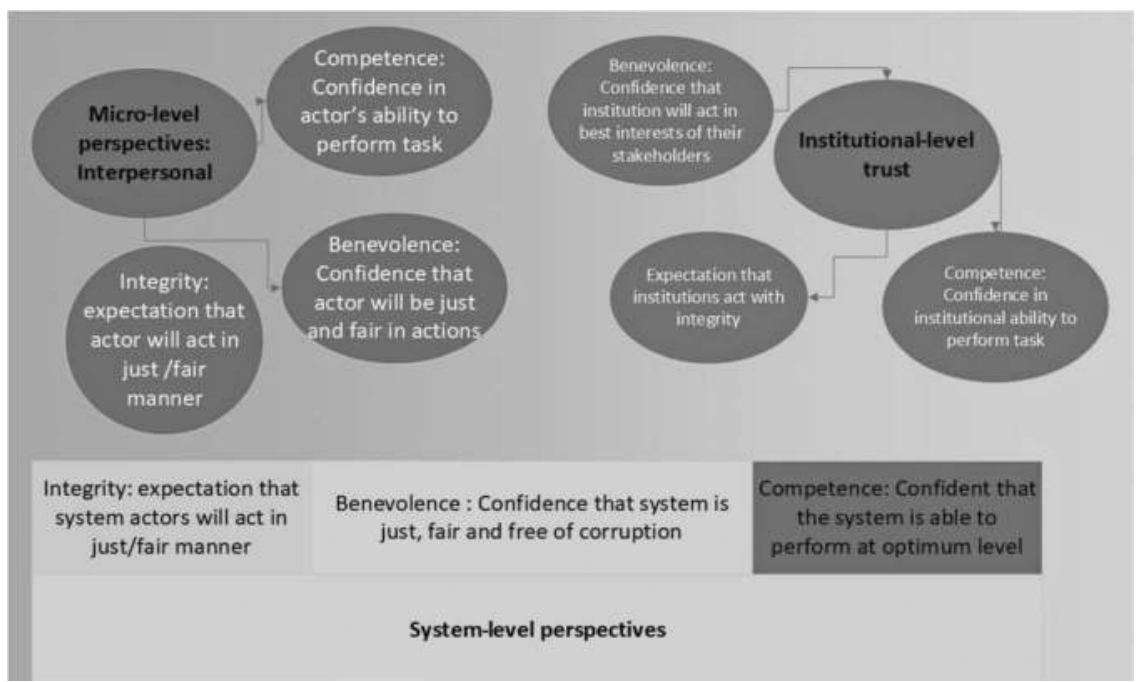

FIGURE 4.1 System Level Perspectives Adapted from Oomsels and Bouckaert (2017b, p. 82)

For example, a parent may trust a school to deliver a good education for their child. This is a risk: if the school does not deliver, it may take a considerable amount of effort and remedial work on the part of the parent, in order to achieve this aim for their child. In such a case, it is likely that the parent will view future interactions with schools with suspicion. This is what economists refer to as a high transaction cost: the less trust that the trustor has in another actor, institution or system, the harder they must work to establish that this individual or entity is to be trusted. This has both a material cost and an emotional one: if a parent lacks trust in a teacher, they will take actions to monitor the teacher's performance (material cost), they will also worry whether this teacher is compromising the learner (emotional/attitudinal cost). This, over time, will result in anger and frustration. In the presence of trust, control mechanisms are largely redundant (Bachmann, 2011; Six, 2013).

High levels of trust in actors, institutions or systems, are likely to increase capacity at each level, as this allows for more efficient collaboration and exchange of knowledge and ideas and thereby increases social and material capital. For example, if a local education authority acts with integrity, benevolence and competence, it is likely that it will be trusted to allocate financial and human support to the schools within its care. The ability to trust in these aspects makes schools feel supported and allows them to plan for long-term improvement in a cooperative manner with the local authority. These feelings of support and trust are then relayed to teachers, who begin to think of the LEA as benevolent and an authority that can be trusted, which will promote their 
willingness to engage with the LEA in improving the quality of their teaching and listen to suggested advice. Such engagement, over time, induces a cycle of high-trust relationships between organisations, increasing the capacity of both, to function at optimal level.

\section{Distrust}

Evidence suggests that in order to fully understand and conceptualise trust it is necessary to conceptualise distrust as a separate construct (Lewicki et al., 1998; Van De Walle and Six, 2014). This helps researchers to examine how certain contexts and cultures can inhibit or constrain the capacity of individuals, organisations and systems to improve or work at optimum level (Keyton and Smith, 2009). In these contexts distrust is viewed as a constraining element, leading to negative perceptions of others' behaviour and limiting successful organisational outcomes (Lewicki et al., 1998). In order to examine how distrusting cultures occur, it necessary to explain what is meant by distrust and how it is distinct from a lack of trust.

Van De Walle and Six (2014, p. 6) argue that distrust has its basis 'in reason, routines and reflexivity that lead to negative expectations towards the actions and intentions of more or less specific others'. They position distrust as distinct from trust in saying that the opposite of trust is an absence of trust, and thus the opposite of distrust is, likewise, an absence of distrust. This means that distrust is not the absence of trust, but an attitude in itself. It is an actual 'expectation that another actor cannot be relied upon, and that they will engage in harmful behaviour'. If distrust is characterised as, 'an actor's assured expectation of intended harm from the other' (Lewicki et al., 1998, p. 446), then we may expect that a culture of distrust (at both organisational and system level) is characterised by 'a pervasive, generalised climate of suspicion' (Sztompka, 1998, p. 22), leading to alienation and passivism. Evidence from research on governance illustrates that distrust influences not only attitudes but also behaviours of people in their encounters with unknown others, as well as how organisations (fail to) engage in multi-agency collaboration. Here the role of boundary spanners, individuals who act as spokesperson for an organisation and manage the collaboration with others, is key. They can disperse distrust and a culture of suspicion across an education system and will likely do so when they themselves are perceived as acting in distrustful ways, such as when they are part of system-wide corruption (discussed later in this chapter) (Richter et al., 2006).

These boundary spanners have a unique place within systems, acting as 'bridges and brokers', facilitating or inhibiting the flow of information (and resources) between organisations and individuals. They can, at best, support the controlled transfer of specialised knowledge between groups, increase cooperation by liaising with people from both organisations, and improve efficiency by introducing good ideas from one isolated setting to another' (Long et al., 2013, 
p. 2). They also have a key role to play in bringing together groups that suffer from mutual distrust (Long et al., 2013, p. 3). This will be discussed further in the concluding section of the chapter.

Groups or individuals who are distrustful of others are infused with negative interpretations of another's behaviour, motives and intentions, proving inimical to effective communication, conflict management and negotiation processes between individuals and organisations (Sitkin and Roth, 2006). Discourses of distrust and powerful institutional and system-wide beliefs of others' ill will, lack of capacity and/or integrity may have their roots in past dealings between groups and may have little relevance in current situations. For example, in South Africa (SA), traditional distrust in teachers, as putting their own protection and their unions before learner well-being, has undoubtedly contributed to negative perceptions of professionalism. In conceptualising teachers in this way, any attempt to promote teacher professionalism is stymied by the belief, regardless of whether it is true or not, in the present system (Baxter et al., 2019).

High levels of distrust between communities, for example between school and district or local education authority, can lead to 'othering' of those that are seen to be different in some way from the target group (Johnson et al., 2004): for example district advisers being seen as controlling, their only function to report poor teaching, rather than being seen as critical friends; there to help teachers improve. This in turn may lead to powerful resistance discourses that undermine or radically alter policy intent (Thomas and Davies, 2005). It can, as a number of researchers point out, lead to extreme passiveness within society; a lack of will or apathy to question the status quo - this is largely brought about due to an inherent belief that 'the system' will not change and that individuals lack both power and capacity to effect improvement (Oomsels and Bouckaert, 2017a). This kind of attitude has been noted in a number of systems in the case of schools in very challenging areas and that also lack good leadership and disciplinary systems: staff are undermined by the constant struggle to make a difference in a culture that is not conducive to improvement, eventually becoming disempowered and lacking in agency - the power to make a difference (Simon and Johnson, 2015).

One of the key elements in the creation of distrusting cultures has been found to be corruption. As in the case of trust, corruption can permeate at micro, meso and macro levels of the system (see Figure 4.1) and create distrusting contexts and cultures that are inimical to capacity and the ability of a system to improve. In the next section, we examine how this works, and why this may be so.

\section{The growth of distrusting cultures: The role of corruption}

Corruption is challenging to define as it is culturally constructed and also has distinct meanings in different disciplines. 'For the anthropologist it is a social relationship, whereas for the political scientist it is a way of governing' 
(Weingrod, 1968, p. 80). Anthropological understandings are linked strongly to sociological understandings, which, for the purpose of this study, are useful for understanding the micro processes that go to make up perceptions and understandings of systemic corruption. For example, the study of patronage, as phrased by anthropologists, is the analysis of how persons of unequal authority, yet linked through ties of interest and friendship - manipulate their relationships in order to attain their ends (Weingrod, 1968, p: 378). This strongly links to the sociological approach in describing discourses of corruption and the ways in which they create hegemonic beliefs that, in turn, create cultures of corruption and distrust. Within the sociological context, perceptions of corruption can be as, or more, powerful as the corruption itself (Clammer, 2012). This is due to their ability to condition individuals' behaviour: for example if individuals believe that no one else is paying tax then it is more likely that individuals will follow suit, whether this belief is erroneous or not. Corruption, in common with other institutional beliefs and hegemonies, is self-perpetuating, becoming, as Bourdieu (1979, p. 34) describes, 'habitus': 'a system of durable, transposable dispositions which function as the generative basis of structured, objectively unified social practices'.

Such dispositions and practices, when viewed as a whole can be referred to as 'culture'; 'an acquired system of habitual behaviour which generates (or/and determines) individual's schemes of action. In short, social structures social arrangements in a society that create order (or attempt to), produce culture - how things are done within society; habits and routines, which, in turn, generate practices, which, finally, reproduce social structures' (Rapport and Overing, 2007, p. 4). A good example of this is the situation referred to earlier, in which teachers were distrusted at one point in history, due to their behaviours at a particular point in time. Over time that discourse remains, thus creating a scenario where it is difficult for teachers to see themselves as professionals - being limited, as it were, to the ways in which they were framed in the past (and present). This is largely due to the ways in which professional identities are formed and conditioned in relation to others and the ways in which actors are perceived by other actors: for example a teacher who is respected by pupils will in turn promote this public persona and their professional confidence concomitantly re-enforced by this positive approbation (Alsup, 2006).

Research has established that corruption in education is incompatible with educational goals, particularly in relation to the aim to produce, 'citizens respecting the law and human rights' (Meier, 2004, p. 2). As Meier and others outline (Hallak and Poisson, 2005; Meier, 2004), it undermines parity of provision and access as well as impacting negatively on the overall quality of provision. The failure of a country to create an effective and meritocratic system of education puts the very survival of that country at risk, by undermining the capacity of citizens to adopt an ethical and transparent way of interacting with individuals and organisations within that society. In short, by destroying or undermining 


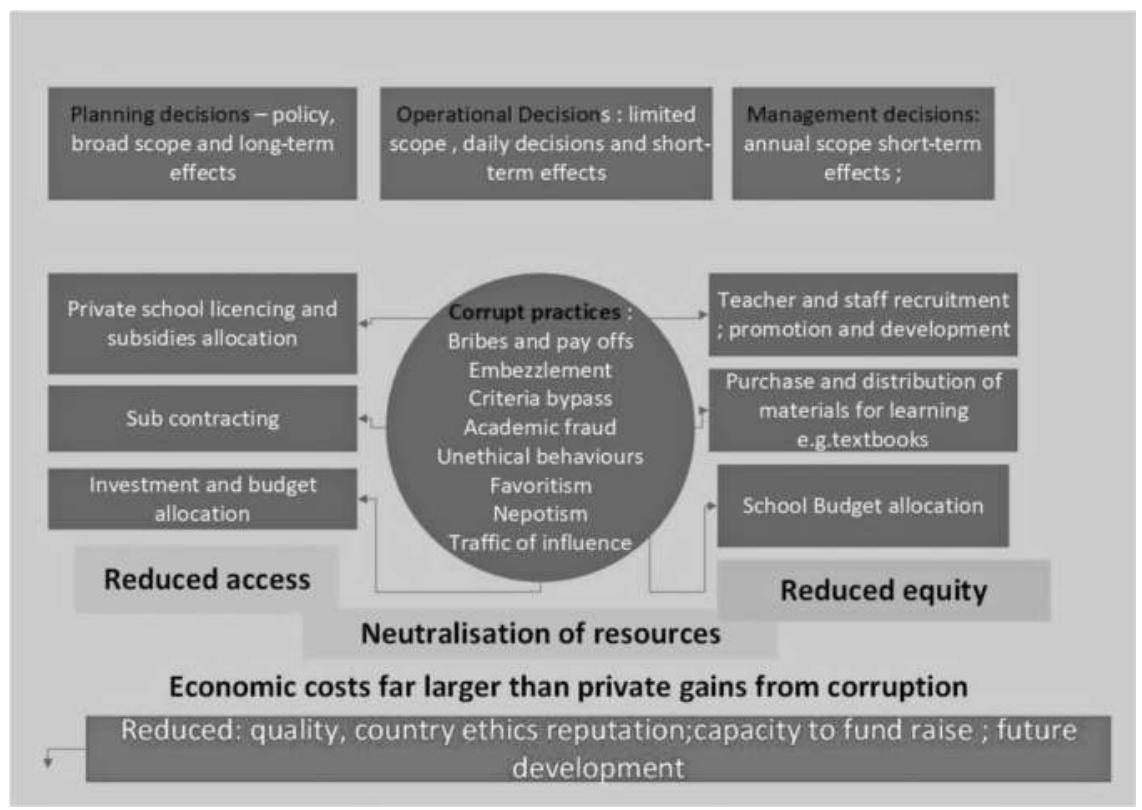

FIGURE 4.2 System wide effects of nepotistic and corrupt practices Adapted from international institute for educational planning: www.Unesco.org

the social capital within that system, corruption thrives in societies with weak systems of accountability, as there are few or ineffective measures to detect and prevent it.

As Figure 4.2 illustrates, the three broad areas of influence exert both long and short-term effects: planning, operational and management decisions, which, in turn, affect educational access, neutralise resources and equity and, finally, impact the reputation of the country in terms of ethics, capacity to fundraise (investment) and future development. Corrupt activities (the central circle) are wide ranging in their capacity to undermine at every level. The effects of these corrupt activities reduce access, neutralise resources (by either misallocating or allocating in ways that are unfair and not conducive to building capacity) and reduce equity. Such practices at micro (individual), meso (organisational) and macro (system) levels undermine trust at every level, each level exerting a domino effect on the other. Figure 4.3 illustrates how this might operate to undermine one of the areas identified by Scheerens (2014) as being core to a successful educational system: 'Achievement orientation and high expectations: a productive school climate, a school mission focused on achievement, shared vision and goals, high expectations that all students can achieve.'

The UNESCO report into corruption in education (Hallak and Poisson, 2001, p. 101) identifies areas in which corruption is likely to arise in education, 


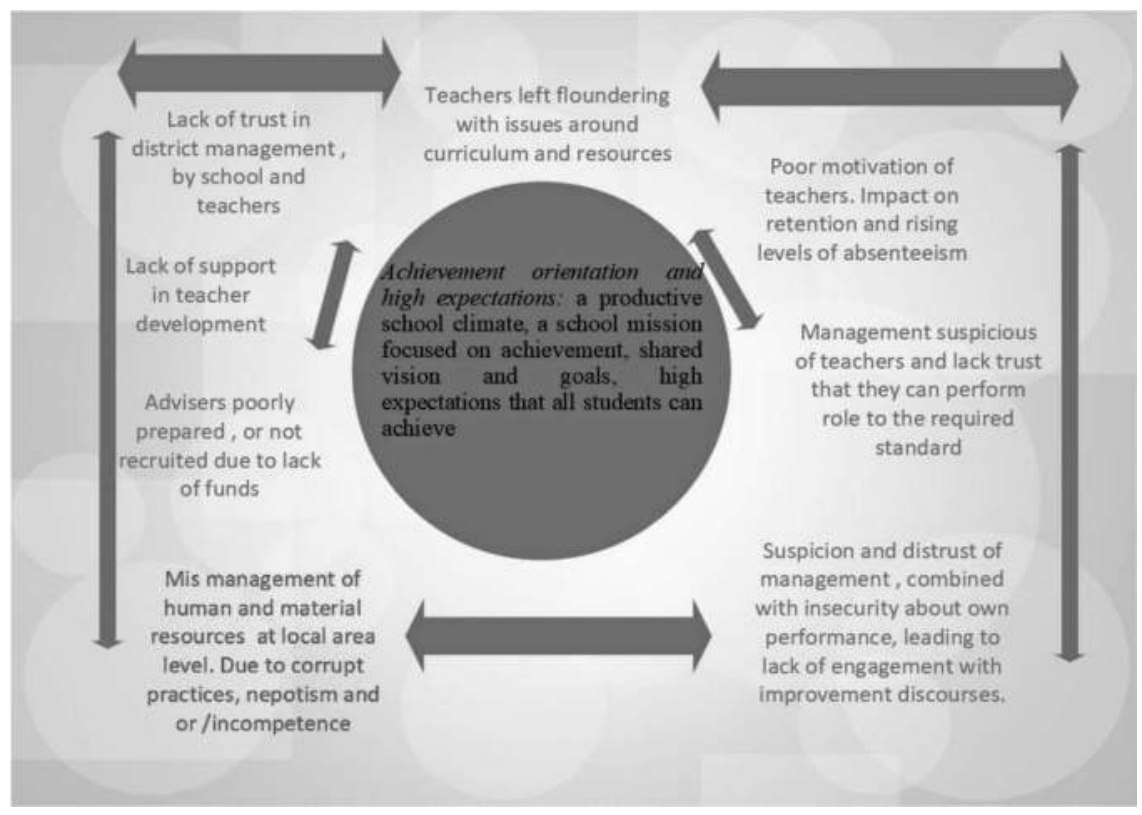

FIGURE 4.3 Mismanagement of resources at local level and consequences

dividing these in to three main areas: planning, operational and management. As the figure illustrates, the consequences are wide ranging and impact educational quality and equity in myriad ways. Once a culture of corruption infuses a system, the impact and consequences are deeply trenchant and resistance to change (Hallak and Poisson, 2005), creating the type of 'habitus', described earlier.

Mismanagement of resources can give rise to perceptions of corruption, for example a regional education department that has no processes for allocating funds to school can produce perceptions of corruption, even if this is just poor management. However, a lack of processes and fair procedures can lead to certain favoured schools being given more funding than others. If both actions have the capacity to produce perceptions of corruption, then it is - by most of the classifications used by Transparency International - corruption: Figure 4.3 illustrates how mismanagement of human and material resources at local authority/district level can create a climate of distrust, which, in turn, impacts negatively on school performance, undermining social and material capital at all levels. Individuals at school level in a system replete with distrust, and with no access to how resources are allocated, are unlikely to see this as mismanagement, and more likely to believe that resources are being appropriated in light of the agendas or ambitions of particular individuals. Such a case illustrates how corruption permeates at system level, impacting downwards on personal and professional relationships 
and upwards at system level. Groups of schools suffering from similar mismanagement can create system-wide distrust, which infuses the system, undermining it at every level.

As discussed earlier, cultures of distrust are heavily influenced not only by leaders within that system, but also equally by boundary spanners. Boundary spanners in education, such as subject advisers, or inspectors of education, can emerge from distrusting passive aggressive cultures to interact with other organisations, spreading these negative cultures throughout a system (Long et al., 2013). They have the capacity to perpetuate discourses of corruption and distrust, or the converse. A discourse, also referred to earlier, draws from the Foucauldian meaning of the word, in that it is a means of constituting knowledge, together with the social practices, forms of subjectivity and power relations which inhere in such knowledges and relations between them (Foucault, 1980). A discourse of nepotism, or favouring individuals with similar political or social affiliations, can also be construed as corruption, for example, in SA, cadre deployment of District Subject Advisers, by certain unions (the deployment of union members in key positions in order for that union to attain power within the system), has had a negative impact; not only on negative perceptions of appointment processes, but also equally on teacher skills and credibility of advisers more generally (Franks, 2014; Twala, 2014). Some deployments of this type are offered to people who lack the requisite skills to perform their role and end up advising teachers who effectively know more than they do. This often leads to those same advisers adopting a monitoring, 'tick box' approach to their work rather than the advisory professional role that would aim to improve teaching and pedagogical skills in that particular subject (a function that they are all too often, simply incapable of performing). This in turn creates a climate of distrust between teachers and their advisers (even though some advisers are very skilled) (Volmink et al., 2016).

As mentioned earlier, distrusting cultures engender mutual suspicion of actors within the system, and so often 'others' particular groups within the system othering is a process of stigmatisation that defines other groups or individuals in a negative way - a facet that inhibits cooperation and intergroup understanding and collaboration (Jensen, 2011). Thus, the chapter argues that distrust and corruption severely inhibit motivation, and, in so doing, capacity to improve, at all levels within a system.

\section{Overturning distrust and restoration of capacity}

Overturning distrust and restoring capacity to a system is not likely to happen overnight, but rather represents long-term efforts at every level of a system. In terms of education, there are myriad ways of gradually infusing a system, over time, with measures that, when viewed cumulatively, may gradually erode distrust, replacing it instead, with trust: leading to higher social and material capital, as individuals become more willing to act in ways which impact 
positively on the system, and organisational cultures improve as a result of this. In order to examine how this can be done, the chapter returns to the Burns and Koster's (2016) four levels of system capacity in education, examining each level in turn in relation to evidence supporting positive changes within system capacity in education.

\section{Individual level}

the means by which to support individuals (parents, teachers, headmasters and policy makers) as they face the demands of new developments in the local context by building on existing knowledge (human resources and knowledge management).

As this chapter has described, there are numerous ways in which the actions and activities of individuals can create or undermine system-wide capacity through micro actions and attitudes that, overtime, permeate and infuse organisations, both within and across institutional boundaries. Evidence suggests that the individual ethical standards of leaders are important in modelling positive behaviours within schools (Fard, 2012), this is particularly powerful when leaders recruit staff that also model similar ethical and professional values (Presley, 2012). Professional values of teachers are also key to what is termed 'professional accountability', in which professional norms and ethics responsiblise professionals as they go about their work (Anderson and Planning, 2005). As Figure 4.3 indicates, corrupt practices include unethical favours, favouritism nepotism and criteria bypass, all areas in which individuals may engage. Professional values and norms, when instilled at an early stage, have been found to permeate the professional identity to such an extent that unethical practices are abhorrent to actors delivering that particular service (Helmich et al., 2017; Wood et al., 2016). Evidence also suggests that modelling behaviours is also a key way to encourage values-based practice, when leaders and key figures within a system model high ethical standards, they 'set the bar', for similar behaviours throughout the system (Engelbrecht et al., 2017).

The ability to critically reflect on practice is an important source of capacity creation, particularly when carried out with others, as a professional development activity. Identifying and then acting on problems, in collaboration with peers, is core to developing good practices, and there is plentiful evidence that shows that in relation to teaching and leadership, this goes a long way to creating positive and proactive developmental cultures (Kuit et al., 2011). It also creates a culture in which mistakes are seen as learning events, rather than something to be penalised, rendering it far more likely that these mistakes can be learning events, contributing to both individual and organisational practices. 
Managers of human and capital resources, such as district officials and actors at provincial levels, also have a part to play: if they take an ethical approach to their work distributing resources in a fair and equable manner, in order to ensure that they reach those schools most in need, this will go a long way to creating a culture of trust at school level. However, this may not be possible if funding arrangements are problematic in the way in which they categorise schools. For example, if funding does not take account of school circumstances, struggling schools in areas of high socio-economic deprivation, such as rural areas, will suffer as a result. In these circumstances, despite apparently ethical behaviour of resource allocators, schools may still be under-resourced (Hall and Giese, 2009).

\section{Institutional level}

The building of learning organisations features as a key marker of school quality: for this to occur, there must be ample support for ongoing development of both teachers and leaders. If this is missing, or lacking at the local level, then the chances of building such a community are much reduced (Giles and Hargreaves, 2006). As Peter Senge, original conceiver of the idea of learning organisations, states, 'in a learning organization, members are constantly and collectively improving their capacity to create and realize a common vision. A learning organization is an organization in which people at all levels are, collectively and continually enhancing their capacity to create things they really want to create. A school's ability for collective learning spells its survival or failure in the 21st century, where interdependence is a must.' For schools to become this type of organisation, 'teachers must trust one another enough to work together and learn from one another' (Senge, 1995, p. 6). In order for this to happen, teachers need to be inducted with the idea that collaborative professional development is a norm and part of professional values. Leaders too have a responsibility to embed this within the organisation, so that it becomes an organisational norm (Bush and Glover, 2003). However, where external accountability demands are excessive or lack real engagement with practice, this is unlikely to occur; as actors will attempt to comply by gaming the system, in order to survive (Ehren et al., 2013). To counter this, accountability mechanisms need to take account of the context and culture in which they are based, in order to attain legitimacy and responsiveness/compliance by the teaching profession (Baxter and Clarke, 2013): For example, in SA, school inspection was used during the apartheid period, to promote a Bantu curriculum - one that promoted white supremacy. To reinstigate inspection in a post-apartheid society has proven all but impossible due to historically negative associations it has with a now discredited system (Deegan, 2005). However, this is not to say that some sort of process could be implemented, for example a system of school self-evaluation system, but this too may be problematic for a school system that lacks the maturity to be able to do this. 


\section{System level}

In order to ensure that distrust is eradicated at system level, it is necessary to ensure that it does not permeate downwards from national government, nor should it emanate from institutions themselves - in this case, schools. In cases in which whole governments are thought to be corrupt, this makes resolving systemic distrust, very difficult indeed (David-Barrett et al., 2017). Appropriate accountability at every level is necessary in order to ensure system capacity remains high. This means holding corrupt practices to account at all levels of the system. Choosing appropriate mechanisms for such accountability is essential, and, as stated earlier, a one size fits all approach will not guarantee success, and context and culture must be considered, if accountability is to add to system capacity rather than undermine it. For example, in England, rapid changes to the education system over the past 10 years have undermined the system of local accountability, which has been in place since the Post War Consensus. The system of inspection too has not changed to take account of new structures, leaving wide gaps and accountability that are leading to mismanagement, cronyism and malpractice in some school groupings - multi-academy trusts, which are groups of schools governed by a single trustee body and led by an executive head teacher (Baxer and Floyd, 2019).

Earlier, the chapter described the role of boundary crossers, within systems, outlining the ways in which they can exert effects which are inimical to capacity and encourage system-wide distrust. However, boundary crossers have also been identified as actors that can break down inter-institutional distrust and militate against widespread distrust (Richter et al., 2006). For example, helpful and well-informed school advisers can broker very positive and trusting relationships between schools and their local education authorities or districts (Reezigt and Creemers, 2005). Unions can bring together schools and staff at professional development events, which aid the sharing of good practice and encourage development of learning organisations (Heystek and Lethoko, 2001; Long et al., 2013). They can also militate against resistance to unpopular policies, by the ways in which they choose to implement them: it is recognised that in the policy implementation chain, such individuals can not only colour the ways in which policies are received, but, equally, influence the extent to which they are implemented in ways which are positive and well received by system actors (Baxter, 2017). School inspectors, for example, can positively influence the ways in which schools self-evaluate, an important skill in which inspection becomes more of a two way conversation, rather than an externally imposed set of tick boxes which schools may struggle to make sense of (Copping, 2011; Vanhoof et al., 2009). If schools are able to adequately self-evaluate in terms of their strengths and weaknesses, it capacitates them to be able to address these, in relation to material and human capital. There is plentiful evidence which suggests that the ability to reflect on issues is key to positive organisational culture development, as actors 
are more likely to admit to difficulties: the first step to addressing them (Avis and Fisher, 2006; Mezirow, 1990).

In 2013, researchers from the Universities of Gothenburg and Lund investigated why anti-corruption measures fail. Focusing their study on two 'thoroughly corrupt countries,' Uganda and Kenya, they concluded that current anti-corruption measures fail because they are premised on the principal agent framework, taking the existence of non-corruptible principals for granted (Persson et al., 2013, p. 450). In other words, many anti-corruption measures fail because there is an implicit belief that there are individuals within a particular society that are not corrupt; therefore, they are capable of holding other (corrupt) actors to account. Persson and colleagues argue that within a systemically corrupt society the short-term benefits of being corrupt are likely to outweigh the costs; therefore, principals (assumed to embody the public interest) and agents (those they hold to account) are both equally corruptible. This thinking is premised on the work of Ostrom (1990), in conceptualising corruption as a 'collective action problem'. The idea that all actors may understand that there is much to gain from not indulging in corrupt practices, but 'because they cannot trust that most other actors will refrain from corrupt practices, they have no reason to refrain from paying or demanding bribes' (Ostrom, 1990, p. 457). In their view, corrupt systems will not be changed by making some actors accountable to other actors, as both are likely to be equally corrupt, but rather to change actor perceptions about the degree to which a system is corrupt: in education, this effectively means that as long as actors perceive that the system is corrupt, corruption will continue. In order to reduce corruption, actors must be convinced that the system is not corrupt and that corrupt actions will be out of line in such a system. This, they argue, is the reason why so many anti-corruption initiatives fail. In fact, Transparency International corruption index judges the extent to which societies are corrupt by the perceptions of actors - under the Ostrom thinking, such an index can only compound the issue. That said, there is no easy answer to resolving such situations. Countries that have successfully combatted corruption have done so by a combination of informal and formal mechanisms that strike at the heart of all major institutions, with members of the ruling elite, holding themselves up as role models of good practice. This approach strongly advocates corruption as a societal issue, which leads us into our final section.

\section{Society}

Education is central to the well-functioning of a democratic society (Freire, 1996), and in order for it to be successful, it must be central to the communities and societies which it serves (Raftery et al., 2012). Distrust between schools and communities undermines capacity, as parental and community support for schools is vital in order for schools to function, as Scheerens (2014) states: 'Parental involvement: parent support, home school partnership', is core to capacity. Where this is weak, teachers' efforts can be undermined, as support for homework, 
arriving on time and behaviour, is key to successful pupil learning outcomes (Bojuwoye, 2009). Pupil absenteeism is a key issue for many developing countries, and poor communities in developed countries, as students are often either called to look after siblings (Christenson, 2004) or do other forms of work. It is also important that a school is supported by the community which it serves must work (Baxter and Cornforth, 2019). Schools create capacity within communities and represent a powerful way for students (and families) to rise out of poverty; in effect, they promote social mobility (Kahlenberg, 2000).

Support for schools, from parents and communities, is however not straightforward. School governing bodies (SGBs) have been seen in many societies as a way in which communities and parents can influence education. For example, SGBs in SA possess more decision-making powers than almost anywhere else in the world (Karlsson, 2002). Members are elected via a public poll every 3 years, and membership comprises parents, teachers, community representatives and the principal (ex officio). Their powers include provision to set and collect school fees (subject to parental approval), determine admissions policies, set languages of teaching, set school development plan (in collaboration with the principal) and establish a code of conduct. However, trust in these governing bodies, by parents, teachers and the community, can be compromised through nepotism or corrupt practices. In SA, most of the major unions recommend that their members stand for election to governing bodies - this includes officers of the union. This effectively means that unions also have considerable influence on the areas outlined earlier. Unfortunately, in some cases, unions effectively 'capture' governing bodies, exerting powerful and, in some cases, hegemonic influence on appointments processes. This influence is compounded if the principal is also a union member or officer (McClure Pattillo, 2012). As governing bodies in SA have the power to set language policy in schools, they have also been accused of ensuring that Afrikaans-speaking white South Africans and their acolytes can determine the language policies, admissions, the types of teachers and the influence of community leaders (especially professionals), which can make public schooling prioritise their cultural and class interests (Volmink et al., 2016). Latterly, moves to remove certain powers from SGBs (for precisely these reasons among others) have led to lack of trust between SGBs and the state at the national level, as can be seen by the response to consultation to reduce the powers of governing bodies via the Basic Laws Amendment Bill (2017). Although this is not strictly corruption, it does impact the equity and accessibility aspects of educational corruption illustrated in Figure 4.3. This leads to widespread distrust of particular schools, in relation to their mission of being able to provide quality education for all.

Corruption is not always intentional; some abuses of power and conflicts of interest can occur if members of governing bodies are not appropriately prepared for the role. Research within a number of countries illustrates this and recommends that governor training and development as a vital element of a well-functioning system (Baxter, 2016; Bush and Heystek, 2003; Moos and Paulsen, 2014). 
Chairs of governance are also key to setting clear standards for a board (James et al., 2011), and modelling high standards of ethical behaviour is a central element within this (James et al., 2012). Boards also need to be made up of individuals with certain skills, combined with community representatives; in this way, boards can function at optimum level, whilst ensuring that schools are invested in communities, and the converse (Roulier, 2000). Lack of knowledge of cultural and contextual issues within school communities, on the part of the school, can leave schools disconnected from real knowledge of students' context, which in turn, erodes social and cultural capital within the school and community.

\section{Conclusion}

This chapter has discussed the link between capacity and distrust and how capacity can be undermined by distrust. Distrust, as the chapter has illustrated, is heavily influenced by corruption and nepotism, and once a distrusting culture is established, it creates a downward spiral of distrust, which then creates more distrust (Figure 4.3). The chapter also discussed how distrusting cultures undermine social capital and resource allocation, and highlighted once distrusting cultures are established, they are highly resistant to change. Corruption and nepotism are difficult to combat, particularly if they infuse entire systems. Yet, as the final section illustrates, there are enabling mechanisms that can be put in place, which can and do begin to erode distrust and enhance social capital: high ethical standards from leaders within the system; professional codes or norms, which reflect high ethical standards of teachers, that are instilled during the training period and refreshed by continual professional development throughout the career of the individual; the ability to critically reflect on practice, fostering a culture in which mistakes are seen as learning events rather than something to be penalised, thus contributing to both individual and organisational practices; fair funding of schools and efficient processes via which to implement funding. At an institutional level, the creation of schools as learning organisations, where staff are constantly and collectively improving their capacity to create and realise a common vision, again fosters the idea that professional development is a norm and an integral part of professional values; leaders, who have responsibility and commitment to embedding this within the organisation; intelligent and realistic accountability systems for schools; for example, it is pointless to judge school on delivery of vast swathes of curriculum, if those holding a school accountable for this do not consider the particular circumstances and the particular socio-economic issues that may be present within the school. In terms of system-level actions that can undermine corruption and promote good practices, governments have a key role to play, in cases in which governments are systemically corrupt, 'the fish rots from the head' the corruption permeates downwards through society. Positive and constructive behaviours from boundary crosses within systems can promote good practice 
throughout, whilst also addressing and calling out corrupt upward practices. As Persson et al. (2013), point out, corruption needs to be seen as a collective action problem, rather than the responsibility of those appointed to hold schools and systems accountable. Parental involvement is key to a well-functioning school and education system. Parent and community representatives must work together to ensure that education is a joint responsibility between school and community, and school and parent.

Most importantly, as the chapter argues, distrusting cultures need to be examined and addressed at system level, due to the permeability of cultures to infuse systems and either enhance or erode capacity at all levels.

\section{References}

Alsup, J. (2006). Teacher Identity Discourses Negotiating Personal and Professional Spaces. New Jersey: Lawrence Erlbaum Associates Ltd.

Anderson, J. (2005). Accountablity in Education. Paris: UNESCO.

Anderson, J. A., \& Planning, I. I. f. E. (2005). Accountability in Education. Paris: International Institute for Educational Planning.

Anderson, J. E. (2014). Public Policymaking: Sydney, Australia.v: Cengage Learning.

Avis, J., \& Fisher, R. (2006). Reflections on communities of practice, on-line learning and transformation: Teachers, lecturers and trainers. Research in Post-Compulsory Education, 11(2), 141-151.

Bachmann, R. (2011). At the crossroads: Future directions in trust research. Journal of Trust Research, 1(2), 203-213.

Ball, S. J. (2007). Education plc: Understanding Private Sector Participation in Public Sector Education. London: Routledge.

Baxer, J., and A. Floyd. 2019. "Strategic narrative in multi-academy trusts in England: Principal drivers for expansion.” British Educational Research Journal 45(5), 1050-1071.

Baxter, J. (2016). School Governing: Politics, Policy And Practices. Bristol: Policy Press.

Baxter, J. (2017). School Inspectors as Policy Implementers: Influences and Activities School Inspectors (pp. 1-23). London: Springer.

Baxter, J., \& Clarke, J. (2013). Farewell to the tick box inspector? Ofsted and the changing regime of school inspection in England. Oxford Review of Education, 39(5), $702-718$.

Baxter, J., \& Cornforth, C. (2019). Governing collaborations: How boards engage with their communities in multi-academy trusts in England. Public Management Review. (in press). doi: https://doi.org/10.1080/14719037.2019.1699945.

Baxter, J., Ehren, M. C. M, \& Paterson, A. (2019). Corruption and trust in South African Education: Perceptions of teachers and school boards. In: Paper Presented at the BSA Annual Conference 2019 - Challenging Social Hierarchies and Inequalities,24-26 April 2019, Glasgow Caledonian University, UK.

Bell, A., \& Mladenovic, R. (2008). The benefits of peer observation of teaching for tutor development. Higher Education, 55(6), 735-752.

Bojuwoye, O. (2009). Home-school partnership - A study of opinions of selected parents and teachers in Kwazulu Natal Province, South Africa. Research Papers in Education, 24(4), 461-475.

Bourdieu, Pierre. 1979. "La distinction: critique sociale du jugement."

Braithwaite,V., \& Levi, M. (2003). Trust and Governance. London: Russell Sage Foundation. 
Burns, T., \& Koster, F. (Eds.) (2016). Governing Education in a Complex World. Paris: Organisation for Economic Cooperation and Development.

Bush, T., \& Glover, D. (2003). School Leadership: Concepts and Evidence. Nottingham: National College for School Leadership. (NCSL).

Bush, T., \& Heystek, J. (2003). School governance in the new South Africa. Compare, 33(2), 127-138.

Cerna, L. (2014). Trust: What It is and Why It Matters for Governance and Education.

Christenson, S. L. (2004). The family-school partnership: An opportunity to promote the learning competence of all students. School Psychology Review, 33(1), 83-104.

Clammer, J. (2012). Corruption, development, chaos and social disorganisation: Sociological reflections on corruption and its social basis. In: B. Manuhuia, B. Hindess, \& P. Larmout (Eds.), Corruption: Expanding the Focus. Australia: Academy of the Social Sciences in Australia.

Copping, J. (2011). 'Tick box' inspections scrapped for private schools. The Telegraph. Retrieved from http://www.telegraph.co.uk/education/educationnews/8612745/ Tick-box-inspections-scrapped-for-private-schools.html.

Cotton, K. (1995). Effective Schooling Practices: A Research Synthesis, 1995 Update. Portland, OR: Northwest Regional Educational Laboratory.

David-Barrett, E., Yakis-Douglas, B., Moss-Cowan, A., \& Nguyen, Y. (2017). A bitter pill? Institutional corruption and the challenge of antibribery compliance in the pharmaceutical sector. Journal of Management Inquiry, 26(3),326-347. doi:10.1177/1056492617696885.

David, R.,Teddlie, C., \& Reynolds, D. (2000). The International Handbook of School Effectiveness Research. London: Falmer Press.

Deegan, H. (2005). South Africa Reborn: Building a New Democracy. London: Routledge.

Ehren, M. C., Altrichter, H., McNamara, G., \& O’Hara, J. (2013). Impact of school inspections on improvement of schools - Describing assumptions on causal mechanisms in six European countries. Educational Assessment, Evaluation and Accountability, 25(1), 3-43.

Engelbrecht, A. S., Heine, G., \& Mahembe, B. (2017). Integrity, ethical leadership, trust and work engagement. Leadership \& Organization Development Journal, 38(3), 368-379.

Fard, S. S. (2012). Ethical leadership and moral intelligence. Oman Chapter of Arabian Journal of Business and Management Review, 34(974), 1-6.

Ferlie, E., \& Ongaro, E. (2015). Strategic Management in Public Services Organizations: Concepts, schools and contemporary issues. Routledge: London.

Flinders, M. (2008). Delegated Governance and the British State:Walking Without Order. Oxford: Oxford University Press.

Foucault, M. (1980). Power and Knowledge. Brighton: Harvester Press.

Franks, P. (2014). The crisis of the South African public service. The Journal of the Helen Suzman Foundation, 74(1), 48-56.

Freire, P. (1996). Pedagogy of the Oppressed (Revised). New York: Continuum.

Giles, C., \& Hargreaves, A. (2006). The sustainability of innovative schools as learning organizations and professional learning communities during standardized reform. Educational Administration Quarterly, 42(1), 124-156.

Hall, K., \& Giese, S. (2009). Addressing Quality Through School Fees and School Funding.

Hallak, J., \& Poisson, M. (2001). Ethics and Corruption in Education. París: IIEP-Unesco.

Hallak, J., \& Poisson, M. (2005). Ethics and corruption in education: An overview. Journal of Education for International Development, 1(1), 1-3.

Helmich, E., Yeh, H.-M., Kalet, A., \& Al-Eraky, M. (2017). Becoming a doctor in different cultures: Toward a cross-cultural approach to supporting professional identity formation in medicine. Academic Medicine, 92(1), 58-62. 
Heystek, J., \& Lethoko, M. (2001). The contribution of teacher unions in the restoration of teacher professionalism and the culture of learning and teaching. South African Journal of Education, 21(4), 222-227.

Holmes, L. (1993). The End of Communist Power: Anti-Corruption Campaigns and Legitimation Crisis (Europe and the International Order). Oxford: Oxford University Press.

James, C., Brammer, S., Connolly, M., Fertig, M., James, J., \& Jones, J. (2011). School governing bodies in England under pressure: The effects of socio economic context and school performance. Educational Management, Administration and Leadership, 39(4), 414-433.

James, C., Jones, J., Connolly, M., Brammer, S., Fertig, M., \& James, J. (2012). The role of the chair of the school governing body in England. School Leadership \& Management, 32(1), $3-19$.

Jensen, S. Q. (2011). Othering, identity formation and agency. Qualitative Studies, 2(2), 63-78.

Johnson, Joy L, Joan L Bottorff, Annette J Browne, Sukhdev Grewal, B Ann Hilton, and Heather Clarke. 2004. "Othering and being othered in the context of health care services." Health communication 16(2), 255-271.

Kahlenberg, R. D. (2000). A Notion at Risk: Preserving Public Education as an Engine for Social Mobility.

Karlsson, J. (2002). The role of democratic governing bodies in South African schools. Comparative Education, New York, Century Foundation Press 38(3), 327-336.

Keyton, J., \& Smith, F. L. (2009). Distrust in leaders: Dimensions, patterns, and emotional intensity. Journal of Leadership \& Organizational Studies, 16(1), 6-18.

Kuit, J.A., Realy, G., \& Freeman, R. (2011). Experiences of reflective teaching. Active Learning in Higher Education, 2(2), 128-142.

Lewicki, R. J., McAllister, D. J., \& Bies, R. J. (1998). Trust and distrust: New relationships and realities. Academy of Management Review, 23(3), 438-458.

Long, J. C., Cunningham, F. C., \& Braithwaite, J. (2013). Bridges, brokers and boundary spanners in collaborative networks: A systematic review. BMC Health Services Research, 13(1), 158.

McClure Pattillo, K. (2012). Quiet Corruption:Teachers Unions and Leadership in South African Township Schools. Connecticut: (BA), faculty of Wesleyan University.

McEvily, Bill,Vincenzo Perrone, and Akbar Zaheer. 2003."Trust as an organizing principle.” Organization science 14(1), 91-103.

Meier, B. (2004). Corruption in the education sector: An introduction. Unpublished Paper, Berlin, Transparency International.

Mezirow, J. (1990). Fostering critical reflection in adulthood (pp. 1-20). San Francisco: Jossey-Bass Publishers.

Moos, L., \& Paulsen, J. M. (2014). School Boards in the Governance Process (Vol. 1). London. Springer Science \& Business.

Muijs, D. R. (2014). Researching Teacher Effectiveness.

Newman, J., \& Clarke, J. (2009). Publics, Politics and Power: Remaking the public in Public Services. London: Sage.

OECD (Eds.) (2011). Governing Complex Education Systems. Paris: OECD.

Oomsels, P., \& Bouckaert, G. (2017a). Interorganizational trust in Flemish public administration: Comparing trusted and distrusted interactions between public regulatees and public regulators (pp. 80-112). In: F. Six \& K.Verhoest (Eds.), Trust in Regulatory Regimes Scoping the Field. The Netherlands, Google Books.

Oomsels, P., \& Bouckaert, G. (2017b). Interorganizational trust in Flemish public administration: Comparing trusted and distrusted interactions between public regulatees and public regulators. Trust in Regulatory Regimes. Edward Elgar Publishing.

Ostrom, E. (1990). Governing the Commons: The Evolution of Institutions for Collective Action. Cambridge, Cambridge University Press. 
Ozga, J., Baxter, J., Clarke, J., Grek, S., \& Lawn, M. (2013). The politics of educational change: Governance and school inspection in England and Scotland. Swiss Journal of Sociology, 39(2), 37-55.

Ozga, J., \& Segerholm, C. (2014). Neoliberal agendas in education. In: S. Grek \& J. Lindengren (Eds.), Governing by Inspection. London: Routledge.

Papakostas, A. (2016). Civilizing the Public Sphere: Distrust, Trust and Corruption. London Springer.

Persson, A., Rothstein, B., \& Teorell, J. (2013). Why anticorruption reforms fail - Systemic corruption as a collective action problem. Governance, 26(3), 449-471.

Presley, D. L. (2012). Leadership Strategies to Shape Organizational Culture.

Raffo, D. M., Clark, L. A., \& Arik, M. (2016). Strategic Responses of Non-Profit Organizations to the Economic Crisis: Examining Through the Lenses of Resource Dependency and ResourcedBased View Theories.

Raftery, J. N., Grolnick,W. S., \& Flamm, E. S. (2012). Families as facilitators of student engagement: Toward a home-school partnership model (pp. 343-364). Handbook of Research on Student Engagement. London, Springer.

Rapport, N., \& Overing, J. (2007). Social and cultural anthropology. The Key Concepts. Londres y Nueva: Routledge). Originally published: 2000.

Reezigt, G. J., \& Creemers, B. P. M. (2005). A comprehensive framework for effective school improvement. School Effectiveness and School Improvement, 16(4), 407-424.

Richter, A. W., West, M. A., Van Dick, R., \& Dawson, J. F. (2006). Boundary spanners' identification, intergroup contact, and effective intergroup relations. Academy of Management Journal, 49(6), 1252-1269.

Rothstein, Bo. 2011. The quality of government: Corruption, social trust, and inequality in international perspective: University of Chicago Press.

Rothstein, B., \& Varraich, A. (2017). Making Sense of Corruption. Cambridge: Cambridge University Press.

Roulier, M. (2000). Reconnecting communities and their schools through authentic dialogue. National Civic Review, 89(1), 53-66.

Scheerens, J. (2000). Improving School Effectiveness. Paris: UNESCO International Institute for Educational Planning.

Scheerens, J. (2014). School, teaching, and system effectiveness: Some comments on three state-of-the-art reviews. School Effectiveness and School Improvement, 25(2), 282-290.

Schon, D. A. (1984). The Reflective Practitioner: How Professionals Think in Action (Vol. 5126). Basic Books.

Senge, P. (1995). On schools as learning organizations: A conversation with Peter Senge. Educational Leadership, 52(7), 20-23.

Simon, N. S., \& Johnson, S. M. (2015). Teacher turnover in high-poverty schools: What we know and can do. Teachers College Record, 117(3), 1-36.

Sitkin, S. B., \& Roth, N. L. (2006). Explaining the limited effectiveness of legalistic "remedies" for trust/distrust. Organizational Trust, Volume 4 Issue 3, pp345-512.

Six, F. (2013). Trust in regulatory relations: How new insights from trust research improve regulation theory. Public Management Review, 15(2), 163-185.

Sztompka, P. (1998). Trust, distrust and two paradoxes of democracy. European Journal of Social Theory, 1(1), 19-32.

Thomas, Robyn, and Annette Davies. 2005. "Theorizing the micro-politics of resistance: New public management and managerial identities in the UK public services." Organization studies 26(5), 683-706. 
Twala, C. (2014). The African National Congress (ANC) and the cadre deployment policy in the postapartheid South Africa: A product of democratic centralisation or a recipe for a constitutional crisis? Journal of Social Sciences, 41(2), 159-165.

Van De Walle, S., \& Six, F. (2014). Trust and distrust as distinct concepts: Why studying distrust in institutions is important. Journal of Comparative Policy Analysis: Research and Practice, 16(2), 158-174.

Vanhoof, J.,Van Petegem, P., \& De Maeyer, S. (2009). Attitudes towards school self-evaluation. Studies in Educational Evaluation, 35(1), 21-28.

Volmink, J., Gardiner, M., Msimang, S., Nel, P., Moleta, A., Scholtz, G., \& Prins, T. (2016). Report of the Ministerial Task Team Appointed by Minister Angie Motshekga to Investigate Allegations into the Selling of Posts of Educators by Members of Teachers Unions and Departmental Officials in Provincial Education Departments. South Africa: Department of Basic Education.

Weingrod, A. (1968). Patrons, patronage, and political parties. Comparative Studies in Society and History, 10(4), 377-400.

Wood, C., Farmer, M. D., \& Goodall, D. (2016). Changing professional identity in the transition from practitioner to lecturer in higher education: An interpretive phenomenological analysis. Research in Post-Compulsory Education, 21(3), 229-245. 


\title{
ACCOUNTABILITY TO BUILD SCHOOL AND SYSTEM IMPROVEMENT CAPACITY
}

\author{
Melanie Ehren and Reinhard Bachmann
}

\section{Introduction}

Accountability systems, particularly in education, often have school improvement as the primary goal. In England, Ofsted's strapline for example has been to be 'a force for improvement through intelligent, responsible and focused inspection and regulation' (Ofsted 2017-2022 strategy). Similarly, test-based accountability in the United States under No Child Left Behind (NCLB) and its successor the 'Every Student Succeeds Act' aims to ensure a quality education for all children by exposing achievement gaps and spurring a dialogue and educational improvement. ${ }^{1}$ Many countries have introduced high stakes testing, school inspections or some other kind of monitoring or evaluation of schools in an attempt to raise learning outcomes. Often international assessments, such as PISA or TIMMS, spurred these reforms when countries would find themselves positioned below average in these league tables. The publication of the PISA ranking in 2000 for example led to the introduction of inspection and assessment in many of the German states. Rankings placed the country 20th in reading, mathematical and scientific literacy among 32 countries, and to address this 'PISA shock', the country introduced standards for measuring students' competencies upon completion of secondary schooling, the introduction of large-scale assessment testing at the end of primary and secondary education and school inspections (Altrichter, in Baxter, 2017; Dedering and Sowada, in Baxter, 2017; Grek, 2009). By 2008 many of the German states however ended or amended these inspections as they were costly and were found to have (too) little effect on school quality. Other countries have however continued to raise the bar for schools with ever increasing targets and consequences for failing. In England in 2013, the Inspectorate of Education for example changed its designation of 'satisfactory' to 'requires improvement' in an attempt to motivate a larger group of schools to change. 
The examples highlight the relevance of a broader question of whether, and how external accountability can improve schools, and how various types of accountability can build or inform capacity of an entire education system. This chapter will first present the evidence of effects and unintended consequences of educational accountability; we will discuss the type of capacity required for accountability systems to lead to school and system improvement, before discussing the role of trust in mediating effective accountability.

\section{Accountability for school improvement}

The question of how accountability systems can improve education has been asked many times in search of 'the best system', but evidence of the effectiveness of accountability systems is mixed at best. Ehren's (2016) systematic review of school inspections shows a large variety of qualitative and quantitative studies finding both positive and negative effects. Positive effects would include schools reflecting on the quality of their school and implement specific improvements to adhere to inspection standards and remedy failure as addressed in inspection reports (e.g. Matthews and Sammons, 2004; Ehren and Visscher, 2006; Penninckx, 2015). A small number of studies report of changes and/or implementation of school self-evaluations in relation to school inspections and how the school's organisational capacity improves as a result (Bubb and Earley, 2008; Hall and Noyes, 2007; Courtney, 2012). Only a small number of studies in England and the Netherlands have analysed changes in student outcomes, reporting either a small improvement in academic subjects for specific student groups (Shaw et al., 2003; Luginbuhl et al., 2009; Allen and Burgess, 2012; Hussain, 2012; Ehren and Shackleton, 2016), or a decline in student outcomes in the year after the inspection (Rosenthal, 2004).

A large number of studies, particularly in England, also report of unintended consequences of inspections, such as when schools narrow their educational practices or try to manipulate the inspection assessment. Koretz et al. (2001) similarly discuss both positive and negative responses to high stakes testing. Positive responses would see teachers providing more instructional time, covering more material or teaching more effectively, where ambiguous or negative responses are harmful for student learning and will lead to invalid increases in test scores (e.g. teaching to the test, narrowing of curricula to tested subjects and content). More broadly, school inspections have also been associated with stress, anxiety and increased workload, while schools designated as 'failing' struggle to recruit teachers and head teachers and see their student numbers decline.

\section{Accountability for system improvement}

Various authors also describe more indirect, system-level, effects of accountability systems in building capacity for change by aligning actions of stakeholders in the education system and their capacity to implement and support school 
improvement. De Grauwe (2007) refers to a 'liaison function' of Inspectorates of Education who act as an agent between the top of the education system (where norms and rules are set) and the schools (where education is shaped and takes place). They liaise between the two by informing schools of decisions taken by the centre and by informing the centre of the realities at school level. The annual and thematic reports, published by many Inspectorates of Education to provide a summary of aggregated school inspection assessments and sometimes of specific policy relevant themes, are a clear example of the liaison role of inspections. These reports and allow national governments to monitor the performance of the education system and adjust their policy to improve system performance. The 2017-2022 strategy of the English Inspectorate of Education, Ofsted (p. 10), for example, explicitly aims to improve outcomes across the system:

However, at the national level, Ofsted should play a role in improving outcomes for young people and other learners across the education, training and care systems. We will achieve this through our national survey reports and research, which highlight what is and isn't working to improve outcomes for young people. That reporting is only possible if our inspectors are exposed to the full range of practice that they can then inform others about. For that reason, we will make more use of the flexibility allowed in legislation to survey and inspect a greater proportion of outstanding schools.

The liaison role of Inspectorates of Education can also include establishing good linkages with other services involved in quality development of schools such as pre- and in-service teacher training, curriculum development, preparation of national tests and examination and identifying and spreading new ideas and good practices between schools. This role becomes increasingly important when school systems are decentralised and accountability shifts from vertical topdown approaches to more decentralised structures or horizontal accountability. In horizontal systems, Inspectorates of Education can have a role in identifying and spreading new ideas and good practices between schools, particularly when ambitious reform programmes are being launched.

Effective liaison of accountability agents on the various levels is expected to improve alignment and coordination across the system. De Grauwe (2007) provides an example of support services who follow up on inspection recommendations or in creating a shared understanding of what high-quality education means across the stakeholders in an education system. Ehren's (2016) work on European inspection systems indicates how inspection frameworks enable educators and administrators to have a common language and set of norms to work towards. Having an external accountability framework for all schools can create consensus across multiple stakeholders and different levels of government of how and what teachers are expected to teach, what children are expected to know or do at the end of each grade or school type and how the school day or year should be organised. Schneider et al. (2017) also speak about the power of educational 
data systems in shaping parental choices, community engagement and public support by equalising what insiders and outsiders know about schools.

Alignment in expectations and norms through the enactment of external accountability and the communication function of accountability standards can build system capacity by reducing transaction costs and creating a more efficient system. Alignment for example creates consistency in how subjects are taught across different school phases, to how teachers' initial training and professional development offers the content knowledge needed to teach these subjects. Looney (2011) explains how the transaction costs, in terms of money, time and lost opportunities, is potentially enormous if systems are not well aligned. Examples are of education and inspection systems that have different aims and include different reforms and evaluation frameworks which create a thick web of overlapping, path-dependent, layered relationships that pose inconsistent and conflicting demands on schools. Ehren and Hatch (2013) describe how such misalignment often occurs when education systems are decentralised and newer accountability arrangements on the local level (e.g. city or district) are added to centralised demands already in place (e.g. at the national or state level). An example they give refers to the United States where the national accountability requirements of the NCLB Act of 2001 have been added onto many local state systems, resulting in mixed messages regarding the performance of schools (Linn, 2005). Pallas and Jennings (2009) for example found that, under the 2009 state and city accountability system, schools in New York city fared well under the state system based on NCLB, but poorly under the city's classification, and vice versa.

Conflicting, unclear or ambiguous demands are problematic for various reasons, most of all in that they tend to motivate schools to respond defensively or seek out the most expedient or obviously acceptable position, preventing them from learning and trying out new solutions. Forsyth et al. (2011) and Daly (2009) argue that conflicting demands destroy trust between educators. An example they offer is of administrators who, when they have to mediate competing demands will find themselves in a position where they are asked to send mixed messages, issue edicts or attempt to explain underlying rationales of external standards which they don't support or have only limited knowledge of. Such a situation may arise when principals are confronted with multiple levels of accountability (e.g. from their school board and the state) where each has a different set of standards and performance targets; or when they are asked to monitor and evaluate the delivery of a standardised curriculum while at the same time are required to support the individual professionalism and morale of teachers. Such conflicting demands have the potential of violating trusting relationships.

Alignment and coordination of policies and practices in line with external accountability standards is, however, notoriously difficult, according to De Grauwe (2008), as it often goes against the sense of independence that many of the involved agencies have, and their differences in opinion about, for example, adequate teaching methods. Services which work towards pedagogical 
improvement, such as teacher training and teacher resource centres, often have different views on what constitutes quality education and how to improve (De Grauwe, 2001) compared with perhaps local governing bodies or national policymakers. For accountability standards to be able to align these actors' efforts towards improvement around a similar set of criteria and norms, they need to accept the standards as valid indicators of high-quality education and incorporate them in their daily practices and in how they support schools in implementing feedback from inspections and assessments. An example of where this was not working comes from Mazibuko (2009) who found that in South Africa (KwaZulu-Natal) there was a lack of clarity in the roles of support offices, districts and inspectors, which hampered the support of schools in using inspection recommendations to improve. Other stakeholders, such as resource centres or school support services, rarely followed up on the inspector's recommendations, which made the inspection a one-off activity with few consequences. A similar example comes from Pakistan where Jaffer (2010) explains how the higher authorities just write 'seen' on the supervisor's recommendations without taking any action. There is no decision on the actions that Inspectorates of Education have suggested for school improvement, and this may well frustrate the school staff as well as supervisors, thus creating an overall sense of inertia and demotivation.

Perfect alignment however is unrealistic, according to Looney (2009), given the complexity of education systems which often have multiple layers and links, operate in diverse contexts and employ teachers and school leaders in a range of experiences and capabilities. She therefore argues that it may be more appropriate to think of alignment in terms of balance and coherence in relationships across standards, curriculum, incentives and assessments and evaluations, instead of aiming for a 'tight fit' between the elements of an education system. A somewhat 'looser fit' would also allow for variety in an education system and prevent schools from focusing on a narrow set of indicators or ignore potential opportunities for improvement and innovation.

\section{Capacity as a precursor to accountability}

Schools' acceptance and use of performance feedback from assessment and monitoring systems is an important mechanism of change across the studies EddySpicer et al. (2016) reviewed, but many studies indicate the high level of support schools need to effectively use feedback and implement improvements, as well as adequate communication and distribution of feedback. Schools (and national policymakers), for example, need support in the interpretation of assessment data and in the implementation of data collection activities (e.g. in monitoring systems) in order to ensure the accuracy of the feedback and the identification of actual weaknesses and lack of resources that need to be addressed. Such support also sets expectations in schools around standards of good education and institutionalises external accountability standards. These examples indicate the need to look at the capacity needed for effective accountability; capacity of 
both educators to respond to accountability, as well as capacity of accountability agents who hold schools accountable for their quality and performance. Here we will focus on the capacity required for external accountability, and particularly inspections and high stakes testing as these are two dominant forms of accountability across the world.

\section{Capacity of educators to respond to accountability}

A discussion about capacity to respond to external accountability is not necessarily an obvious one to have. Many countries position high stakes testing or inspections as external measures which are expected to provide an unproblematic, valid and reliable assessment of a school's or teacher's performance on a set of standards. In the discourse about validity and reliability of such assessments, schools and/or teachers are positioned as 'objects of measurement' which are expected to continue their work 'as normal' when for example visited by an inspector. Changes to how the curriculum is taught or how the school is being run would potentially invalidate the assessment as it would measure a fabricated reality. School capacity is viewed in terms of educational quality or the ability to improve, not on their competence to prepare for an external assessment. In England, Ofsted for example purposefully introduced 'no notice' inspections in 2012 to prevent schools from preparing extensively for an inspection and present a 'dressed up', and potentially more positive picture of their school; in the United States, test coordinators, test manuals and test security protocols and procedures are put in place to prevent and detect any irregularity in academic testing.

The reality is however that all external evaluations require some capacity of educators in the preparation for inspection or high stakes testing. On the day of an inspection, inspectors expect to see certain lessons, have access to a range of documents (e.g. minutes of meetings or student work) and often need to interview parents, teachers, students and the principal (Ehren, 2016). A similar level of organisational capacity is needed for the administration of high stakes test which requires a reorganisation of the school week, ensuring parents, teachers and students are prepared for the test and know what to expect, and that students have the stamina and motivation to sit for tests that may take up to 3 hours in each subject. ${ }^{2}$ Although assessments and monitoring systems are generally developed externally, there is an assumption that teachers and head teachers are familiar with implementation requirements of these systems in order to ensure that test scores and management data is accurate, valid and reliable. Teachers and principals would need to understand what is tested in terms of curriculum standards and skills, and be able to prepare their students for the test in such a way as to allow for an accurate measurement of their actual knowledge and skills in the tested domain.

Where accountability systems make use of school-internal evaluation or data systems to inform the assessment of school quality, the expected capacity of educators to respond to external accountability goes beyond mere organisational skills. 
Eddy-Spicer et al. (2016) and Ehren et al. (2013) explain how schools need the ability to evaluate their own performance when external inspections are linked to school self-evaluation. Various Inspectorates of Education (e.g. in England and the Netherlands) draw on school internal data to decide on which schools to inspect and to vary the frequency and intensity of their visits according to schools that are most likely to be failing. These so-called risk-based models assume that schools have the capacity and skills to evaluate their own quality and provide an accurate and honest account of their strengths and weaknesses. When schools lack the time to interpret and use data, they will not be able to provide an accurate account to an external inspection agency, nor will they be able to incorporate findings from external accountability in their ongoing action and improvement planning. Without the skills to evaluate, there is a danger that schools treat the self-evaluation process as superficial and see it as a compliance exercise rather than genuinely aimed at school improvement (McNamara and O’Hara, 2012; Hall and Noyes, 2007).

Schools thus need the competencies to design and implement valid and reliable assessments, the skills and capacities to interpret data from assessments as well as the knowledge and resources to monitor school/system-level processes and school effectiveness conditions and act on feedback. Such 'evaluation literacy' allows school staff to measure their own quality and use evaluative information to improve and to implement internal assessment practices to monitor and improve student learning, to implement external standardised assessments and have systems in place which for example record student and teacher attendance. Lasky et al. (2008), furthermore, point to the need for skills that allow for intentional and possibly critical conversations anchored to student data that can inform teaching and school improvement plans.

Although external accountability can provide a strong impetus to develop such evaluation literacy, it would seem that there must be a balance between external accountability and challenge and support for capacity building to inform transformational change (MacBeath, 2004). Ehren et al. (2015) found that expectations and educational standards set by an inspection framework in the Netherlands not only helped to drive internal evaluation and school improvement, but they also found, in a minority of schools, that there was a narrowing of the curriculum and 'window dressing' of reports sent to the inspectorate to present a favourable picture of the school. In England, internal evaluation is often seen as an internal version of inspection as in some of the schools investigated by Hall and Noyes (2007). In these schools, senior leaders copied the inspection practice in making unannounced classroom observations and judgements against inspection criteria. Teachers who expressed different views on quality teaching as those in the inspection framework tended to be viewed as out of date or incompetent.

Genuine improvement from internal evaluations requires a more supportive relationship between teachers and school leaders, according to MacBeath (2004), where schools often also need external support to improve. Grek and 
Ozga (2010) and Plowright (2007) refer to professional development, support from resource centres as important conditions to improve the educational experience of students in the longer term, according to Grek and Ozga (2010) and Plowright (2007). As these authors explain, capacity must be built within the school to ensure they become learning organisations with ongoing collaborative discussion and decision-making (Marsh and Farrell, 2015; Simons, 2013).

\section{Capacity of test developers and Inspectorates of Education}

The need for evaluation literacy equally applies to those agencies which hold schools and teachers accountable. We often assume that they have the capacity and knowledge and skills to inspect schools or develop high-quality tests which provide a valid and reliable account of school quality and performance. In reality, however, many of these agencies have faced budget cuts and/or are unable to assess school quality accurately. Risk-based inspection models, such as in the Netherlands and in England, are instigated by the need to reduce the number of visits to schools and target inspections to potentially failing schools, while Ofsted has seen mounting critique of how it judges quality of schools in disadvantaged areas. Hutchinson (2016) for example shows that schools in England with a disadvantaged intake or with a high proportion of pupils with low prior attainment are 5 times more likely to be rated 'inadequate' by Ofsted than those with better intakes, and less than half as likely to be rated 'outstanding'. Her data suggest that the inspection framework, in an attempt to evaluate practices and behaviours similarly across schools, actually favours the type of practices found in middle and upper class schools. These schools would for example have a certain type of safeguarding protocol that would lead to a positive judgement of a school's climate, where schools serving disadvantaged children would have a different set of practices in place that, although more fit for purpose, are not valued by the Inspectorate.

Concerns over capacity to inspect schools in developing countries are reported in detail by Eddy-Spicer et al. (2016) and Ehren et al. (2017) who talk about the lack of resources, inefficient management and an organisational structure of Inspectorates of Education which is not fit for purpose. De Grauwe (2001, 2007) and Dembélé and Oviawe (2007) highlight how, in Africa, Inspectorates of Education face a high school-to-inspector ratio, which means that one inspector has many schools to visit, resulting in a high workload where many schools go uninspected for years. The high workload of inspectors in parts of Africa is also informed by the multitude of their tasks, which include, in addition to the evaluation and monitoring of schools, often also school management, support and administrative tasks (e.g. checking student numbers in schools).

Eddy-Spicer et al. (2016) further describe how, in low and middle-income countries, many national assessments fail to meet required technical standards relating to instrument design, sampling, test administration, data preparation, data analysis or data interpretation. As a consequence, the data obtained may 
provide inaccurate information, making its use for immediate policy purposes or for monitoring over time problematic. They also refer to cases in which data had not been made available to educational planners and managers or was not analysed. A common problem in the low and middle-income countries in their review was that reports from standardised assessments (e.g. school report cards or aggregated school-level test scores) were not written in a user-friendly language where users of those reports lacked the technical skills and critical capacity to interpret results.

Similar issues are reported in more developed countries. In the United States, high stakes are criticised by Darling-Hammond (2004) who explains that 'annual yearly progress' targets on standardised state tests was based on the notion that $100 \%$ of students will score at the 'proficient' level on state tests by the year 2014. Given that these tests were norm-referenced where test takers are compared and ranked in relation to another and $50 \%$ of students by definition must score below the norm, it is impossible to attain 100\% proficiency levels for students. Even if tests were not constructed in this way, the steepness of the standard is unrealistic, particularly for schools serving disadvantaged children, according to Darling-Hammond.

Many systems have started to report some kind of value-added measure to address concerns about the accuracy of judgements about schools' and teachers' performance. These measures compare a child's performance with that of children with a similar prior performance and similar circumstances; they aim to take into account the circumstances of children attending the school which are beyond the school's control. The complexity of these measures however raises other concerns, such as over the capacity of educators and their stakeholders to interpret and use these results. According to Harris (2011, p. 10), policymakers in the United States have developed a greater awareness of the need to use value-added measures to evaluate schools, but incorporating these measures into accountability systems will only have a positive impact on schools if the policymakers in charge of designing accountability understand the tools they are working with and if educators, as the object of accountability, understand the meaning of the measures intended to capture their performance and guide their careers. To quote Harris (2011, p. 10): 'nobody will respond well to performance-based accountability if it is neither trusted nor understood'. The high technical detail of value-added measures however has caused some policymakers, such as in England (Leckie and Goldstein, 2017³) to return to simpler, but less accurate, measures, suggesting that capacity to understand these measures is not straightforward.

However, even accountability systems which use value-added measures will not provide a perfectly accurate assessment. As Leckie and Goldstein (2017) explain, schools tend to be inconsistently effective when considering different outcomes, different cohorts over time or when teaching specific groups of students (classified for example by ethnicity, previous attainment and socio-economic status (SES)); some schools are particularly effective for promoting the progress 
of low-ability students, but not high-ability, or vice versa. The need to provide an accurate judgement of school quality, using a standardised framework that doesn't take into account specific school, teacher or student context, poses a set of constant challenge which require a high level of capacity and expertise to be resolved.

In the next sections, we will turn again to the writing in previous chapters on trust to understand how it enables effective accountability for school improvement, as well as system change.

\section{Trust as the enabler of effective accountability (school and system improvement)}

In sections 5.2 and 5.3, we presented the evidence on how external accountability (inspections and high stakes testing) can improve schools and build system-level capacity for high-quality education when inspection, monitoring and assessment operate by setting expectations, providing feedback, involving stakeholders in accountability and improvement and capacity building. These mechanisms can reinforce each other when inspection feedback for example supports the setting of expectations around inspection standards or when the setting of expectations has created an education system in which the discourse is dominated by the logic underlying inspection models and the school's stakeholders have shaped their thinking and activities accordingly. For these mechanisms to 'trigger', trust between schools and the external accountability agent providing the feedback is required, as well as trust in the overall organisation exercising the accountability, such as an Inspectorate of Education or testing agency. The first is a type of relational or inter-organisational trust, where the second is generally described as macro-level trust which would ensure that the accountability agents are seen as legitimate partners in setting standards of quality and in providing feedback which will lead to improvement.

\section{System trust for effective accountability}

Macro-level trust, also called system trust or institutional-based trust (see Bachmann, 2001; Beugelsdijk, 2005), is an abstract concept which arises from the institutional environment of laws, norms, and standards and abstract relationships. Beugelsdijk (2005) explains how the concept is closely related to the well functioning of institutions, such as the functioning of (legal, political, economic) bureaucratic systems, and is set apart from the interpersonal, exchange relationships between individuals and organisations in which trust is often discussed. System trust is, according to Bachmann and Inkpen (2011) and Fledderus et al. (2014), similar to the notion of institutional-based trust and a particularised form of trust in a specific service provider or government agency, such as an Inspectorate of Education or testing agency.

Macro trust can build trust in inter-organisational relationships such as between schools and inspectors or other accountability agents, as well as between 
inspectors and other stakeholders in the system. Bachmann and Inkpen (2011) and McEvily et al. (2003) conceptualise macro or institutional trust as an organising principle to coordinate expectations and interaction between individuals or organisations and where these interactions are viewed as embedded in the institutional environment. Institutions are not just seen as an individual organisation (such as Inspectorate of Education or testing agency) but also as a functioning rule or law, political system or bureaucracy or, in our case, an operating accountability framework. Various authors explain how and when these institutions are trusted to perform the liaison function we are interested in, institutionalise accountability standards and motivate schools and stakeholders to act on accountability feedback. Honesty, impartiality, fairness and legitimacy are key words authors use.

\section{Fairness and legitimacy as conditions for system trust}

Fairness and legitimacy, when outlined for governments and the state, refer to a perception of citizens that all relevant interests are considered or 'that the game was not rigged' (Levi, 2003). In England, Ofsted's phrase to 'inspect without fear or favour' is an example of how this aim applies to accountability systems. Fairness in a regulatory context also refers to how offenders are treated. Gunningham and Sinclair (2009b) for example explain how, in the mining industry, prosecution against those who neither intended harm nor were reckless in their behaviour was widely perceived to be unjust, and how this caused the law to lose its legitimacy in the eyes of duty holders. A similar argument underpins the idea of 'responsive regulation', a concept developed by John Braithwaite and others. Braithwaite $\left(2010^{4}\right)$ explains how regulation needs to be responsive to the moves regulated actors make, to industry context and to the environment by having the capability to escalate to tough enforcement or enhanced support. Responsive regulatory theory started out as a theory of business regulation but has now been applied to many other areas of private and public governance applications as well as education; it offers a powerful set of principles to understand how the legitimacy of regulatory systems can be improved by restorative, dialogue-based and supportive approaches to enforce compliance to regulation, and move towards more punitive sanctions when dialogue fails.

\section{How fairness and legitimacy promotes school and system improvement}

Perceived fairness of accountability systems seems to promote alignment and coordination along their standards in three ways: (1) by creating a stable environment of shared standards for educators to interact in, (2) by promoting compliance to these standards and (3) by increasing group identity and affiliation. The process operates both on the individual level as well as on the organisational level. 
Rothstein (2013) for example explains how it is trustworthy, uncorrupted, honest, impartial government institutions that exercise public power and implement policies in a fair manner which create social trust and social capital. Accountability agents who detect and take care of people and organisations which act opportunistically create assurance for others who have to engage with these individuals and organisations. By setting a clear set of standards and enforcing those in a fair, independent and transparent manner, accountability agents create a stable environment of institutionalised incentives, sanctions and regulative norms which ensures that schools behave trustworthily. Submitting schools to the same norms and incentives ensures clarity over roles, responsibilities and what to expect in a relationship (both between schools and the accountability agent, but also in the wider system), allowing those relationships to further develop.

Perceived fairness motivates compliance, as those who perceive that they have been treated fairly are more likely not only to trust the regulator but also to accept its decisions and comply with its requirements. Gunningham and Sinclair (2009a) refer to Taylor's procedural justice theory to explain that people are more likely to obey rules if those rules are considered to be legitimate and moral, if the process by which such rules are made and applied are considered (amongst other things) fair and honest, and if they are treated with dignity and respect, and have the opportunity to participate. According to Jackson et al. (2012), the legitimacy of the police and the law leads to a respect for legal guidelines for action that dictates appropriate and personally binding behaviour. These guidelines may not be perfectly aligned with everyone's moral system, but legitimacy involves the public recognition that the social order needs a system of laws that generate compliance and respect above and beyond individual preferences (or disagreements) concerning specific laws. When people feel they are treated fairly and acknowledge that it is morally just to obey the law, rule compliance is likely to be voluntary because people feel a moral obligation to comply. The best way to motivate rule compliance then, is by nurturing legitimacy and morality.

In 2016, 40,000 parents signed a petition in the United Kingdom calling for a boycott of primary school tests ${ }^{5}$; the example indicates a clear loss of legitimacy in the high stakes test where the excessive number of tests is not seen as a fair way to assess school quality, or as measures that are in the best interest of children. Rothstein et al. (2009) similarly explain how a diverse and bipartisan coalition of Americans has bemoaned the narrow test-based accountability plans which fail to tell the states and nation whether schools and related public institutions are performing satisfactorily and to indicate where improvements are required. These policies have been widely critiqued for creating incentives for educators to shift effort and resources away from a wide set of educational goals and towards instruction in reading and math exclusively, undermining schools' mission to also raise achievement in other academic areas and in the social, behavioural and civic goals of education. Accountability systems need to be trusted to have 
an effect, as they are equally capable of destroying trust; a topic we'll explore in this section.

As Eddy-Spicer et al. (2016) explain, for high stakes testing in low and middle-income countries, trust in the authority (and particularly their 'pedagogical competency') is an aspect of reasoning that precedes teachers' and school leaders' abilities to use the exam as a means of improving student learning and an important condition for aligning what is taught with what is assessed. Given the right conditions, trust in the authority of the assessment may lead to teachers' engagement with the principles underlying the design of the examination, the development of teaching knowledge and skills that go beyond performance alone and the cultivation of instructional leadership. Such high levels of trust will even ensure teachers and school leaders who oppose an emphasis on testing to continue to be involved in the exam process as they trust and appreciate the potential longer term benefit to their students' knowledge and abilities, particularly when there is limited pressure for high exam results (Yaeger et al., 2012).

Ehren et al. (2017) point to the lack of status, credibility and authority of many inspection systems in low and middle-income countries as an explanation for why inspection feedback is not used. Various authors (Churches and McBride, 2013; Darvas and Balwanz, 2014; De Grauwe, 2001) point to inspectors only doing a simple checking and control of administrative protocols in schools, due to work overload and how they are performance managed by number of visits (instead of impact and quality of feedback). Their lack of professionalism, lack of training in evaluation of school quality and dismissive attitude and sense of inflated power over schools result in a lack of appreciation of their work, poor relationships and a lack of legitimacy for the accountability system overall.

In England, Ofsted has similarly been criticised for its narrow focus on measuring academic outcomes where the new chief inspector, Amanda Spielman, organised an extensive consultation on Ofsted's new (2019) inspection framework to ensure a fair and widely accepted framework. The consultation ${ }^{6}$ highlighted the concerns of 1500 teachers, head teachers and other stakeholders in the system on specific indicators in the proposed framework, as well as wider learnings about what works well in the inspection process. In response to the consultation, Ofsted made a number of changes and explained these in extensive response to consultation documents, such as scrapping the on-site preparation of the visit by inspectors or how bullying is assessed. ${ }^{7}$

Individual experiences of fairness and perceived legitimacy seem to contribute to feelings of shared group membership with the authority concerned and can, as such, promote more indirect mechanisms of alignment around a set of accountability standards. Jackson et al. (2012) and Rothstein (2013) explain how the experience of procedural fairness - including respect and fair decisionmaking - fosters feelings of shared group membership with the authority concerned. Institutions perceived to be fair seem to increase group identity and affiliation so that the goal of the group merges with the goal of the individuals. The underlying mechanism is one of social identity where people who feel they 
are treated fairly identify with the rules and values of the group and feel a moral obligation to adhere to these. As accountability frameworks and resulting judgements of quality and performance communicate information about the status of individuals or individual organisations within a group, they shape identities and communicate status-relevant information. When people and organisations feel they have been treated fairly and respectfully they tend to use these evaluations to gain self-knowledge, construct their personal identities and engage with the groups and its values.

Fair and legitimate accountability systems are also likely to endorse a process of coercive and mimetic isomorphism and create normative pressures to confirm to these standards: a process that is described by Ehren (2016) in the context of school inspections, who talks about how the consequences of inspections and their communication of standards create a process whereby schools model themselves after these standards and after schools which are considered to be successful in responding to the standards. The publication of good practices, league tables of high-performing and failing schools and benchmarking schools on common indicators of 'good educational practices' and outcomes, allows schools to copy protocols, structures and good practices from schools considered to be high performing and provides legitimacy to such practices. Accountability frameworks provide firms and associations, as well as professional networks and training associations, with a lens to identify successful models and practices and change how actors make decisions, do their jobs and think about their schools, particularly when external stakeholders have power and use rankings and assessments to inform their choice of, or funding of schools and when schools face legitimacy threats when for example classified as failing and risk losing funding.

Jackson et al. (2012) and Mansbridge (2014) however also argue that compliance stems from personal commitment to law-abiding behaviour where 'trustworthy individuals' comply with the law because they believe it is the right thing to do. Schools would follow-up on inspection feedback or aim to improve their performance on a high stakes test because they are intrinsically motivated to do so. This mechanism obviously only works when there is a culture of law abidance. Given that such a culture is not likely to be prevalent across all schools, many accountability systems have some type of consequence (e.g. fines or naming and shaming) to enforce of compliance of those who are unwilling to do voluntarily.

\section{Inter-organisational trust for effective accountability}

The boundary spanning function of accountability agents, i.e. inspectors, is essential with regard to building capacity in the education system. Earlier we have already explained how this function could support the coordination and alignment of interaction and expectations in the education system and that collectively accepted standards, curricula, assessments and incentives can create a coherent approach to improvements. To be able to fulfil such a boundary 
spanning role, accountability agents need to be trusted actors who are expected to actively promote their standards so that other stakeholders (e.g. head teachers, parents) would follow their advice and feedback.

Boundary spanners are always important when trust is concerned, and accountability agents play a specifically pivotal role in the education system in that they can bridge over the different perspectives of various actors and thus foster trust among the stakeholders such as parents, regulators, teachers, government and local communities. Trust can spread in the education system, as in any social system, if the exchange of knowledge is effectively and credibly facilitated by key agents, such as inspectors, who enjoy authority and a high reputation for their impartial judgement. They can then function as 'third party guarantors' (Coleman, 1990) in relation to a number of dyadic relationships between other stakeholders in the education system. This means that two stakeholders who do not know much about each and/or have not much reason to invest trust in their relationship (for example government officials and parents) will do so because they both trust a third actor, i.e. the inspector, who is held in high esteem by each of them. If this happens multiple times, it will eventually lead to the creation a trust-based system of social interaction between all stakeholders which allows for openly sharing information, minimising transaction costs of collaborative efforts and contributing to innovative initiatives.

Accountability agents, however, are not simply enabling and enhancing trust but are themselves dependent on some prior form of trust and goodwill on the part of those who they report to and those who they appraise. Without such initial levels of trust, they would not be able to collect information on the quality of teaching and learning in schools as teachers and head teachers would try and prevent their access to the school, or would try and stage a performance disallowing them to understand the actual quality of the school. In such a situation, they would fail to provide feedback on improvement, both to the schools they are supposed to assess, or offer valuable information to other stakeholders in the system; containing their boundary spanning role in the system. Inspectors and Inspectorates of Education thus need to enjoy a certain level of initial trustworthiness and credibility from other stakeholders to allow them to share potential institutional weaknesses and be appraised on them. If this is all in place, the inspector can have a boundary function by spreading trust and openness. The mechanism would operate through the type of mimetic process discussed earlier: inspectors who offer constructive advice to teachers, who use it for improvement will, when witnessed by parents and head teachers, engender trust in the entire system. All stakeholders see that the system is not 'rigged' and that there is benevolence and integrity on all sides to work towards a better public good. In such manner, inspectors can trigger a dynamic process of trust creation that all stakeholders will benefit from.

For inter-organisational trust to develop into such a 'virtuous circle', it is also a precondition that all stakeholders have clarity over each other's expectations and specific interests. This creates a certain level of predictability and ensures 
that stakeholders can see each other as reliable partners. Such predictability typically comes from the inspection frameworks which standardise how schools are inspected (e.g. through various types of data collection and analysis) and the criteria by which their quality is assessed. These standards allow schools to understand how they are being evaluated and when as well as how they can prepare for an inspection, preventing unpleasant surprises and a feeling of unfairness for being judged on criteria that were unknown to them.

Fairness and legitimacy are particularly important to develop a stronger form of trustworthiness and trust, i.e. 'benevolence-based trust' (Mayer et al., 1995). Benevolence-based trust as described by Mayer et al. is particularly important for the inspection frameworks to be used by all involved for the improvement of education, allowing for open conversations between inspectors and school staff about the way in which teaching and learning can improve. If there is no or not enough benevolence-based trust between schools and inspectors, there is a considerable risk that head teachers and teachers will erect a façade for the inspection and focus on ticking the 'right' boxes rather than allowing a substantial and meaningful inspection. In this case, it is impossible for the inspector to fulfil his/ her task properly. This could trigger a 'vicious circle' or downward spiralling process towards ever lower levels of trust, such as when inspectors or inspection organisations respond by penalising schools or aggressively enforcing schools to comply to their framework, thereby promoting even greater levels of distrust towards the inspection and vice versa. It would also incapacitate the inspectors and Inspectorate of Education's overall legitimacy as a boundary spanner across the system; they would simply not be able to collect and disseminate information about the actual quality of schools and the system as a whole. Without such a boundary spanning role, they would not be able to promote inter-organisational trust between schools and other stakeholders in the system who rely on information about school and education system quality to inform their work (e.g. local municipalities in charge of school buildings or improvement support services, teacher training colleges, districts).

Inter-organisational trust may in some cases need some time to develop but will do so when organisations can make positive experiences in interactions with each other. This proves necessary particularly when head teachers, teachers and parents know very little about how the Inspectorate works, what its role is and how it ensures that high standards of fairness are followed. It is important that stakeholders, in order to suggest themselves as serious cooperation partners, can observe sufficient internal homogeneity, commonality and external connectivity when assessing each other's trustworthiness. As we have discussed above, the latter is highly intertwined with alignment and coordination effects produced and rendered by macro-level trust, i.e. system trust. Again, the accountability agent plays a specifically important role in that he/ she, more than any other stakeholder, can provide common ground and an agreeable playing field for all other stakeholders and thus promote a significant level of system trust. 
Accountability agents, by the very nature of their work, are likely to have considerable power by way of their social position within the education system and the capacity to influence narratives and discourses among its key actors. This is does not do harm to trust development but is in fact necessary for inspectors to their role as facilitators of trust (Bachmann, 2001) and to promote good practice in the relationships between other stakeholders. Only if they appear as strong actors, who also can claim legitimacy and are perceived to maintain fairness among the various other actors, they can credibly suggest commonly accepted standards and criteria of teaching and learning and thus play an essential role in trust building and capacity development within the education system. Of course an important condition is that they are fair in how they assess schools and don't benefit their power for personal gain, such as by offering schools bribes to ensure a positive inspection outcome.

Noteworthy, it is not only inspectors that function as boundary spanners. In principle, all stakeholders have external relations and they all play an important role in creating inter-organisational trust. Other examples of boundary spanners include teachers who have earned respect and trust from their peers for their effectiveness in the classroom, or parents who serve on parent-teacher councils or site leadership teams (Forsyth et al., 2011). A further example are district officials who promote or inhibit trust within the education system as they move between institutions and stakeholder groups. These agents sustain connections between the different communities of practice within and across their organisation and are thus a precondition of effective and successful education systems (Millward and Timperley, 2010). All these actors who are specifically active in transcending boundaries can contribute to establishing indispensable linkages among the various stakeholders. The inspection standards however often dominate and inform these linkages as Ehren et al.'s (2015) comparative research on school inspections indicates. Their study highlighted how inspections 'set expectations' across an education system about what quality education constitutes and thereby inform the work of various stakeholders in an education system. As such inspectors and inspection agencies not just function as a boundary in and of themselves, but also inform the work of other boundary spanners, managing the exchange relationships between organisations, aligning informal commitments, explicit and tacit expectations and interests between the stakeholder partners around values and standards underpinning inspection frameworks.

Over time, such alignment contributes constitutively to institutionalise these commitments and norms as well as help transform them into established and taken-for-granted organisational structures and routines. These structures and routines, in turn, channel the orientation of individual members of stakeholder organisations as well as the collectively held orientations towards the partner organisations (Bachmann and Kroeger, 2017), thereby fostering the development of trust in multiple dyadic relationships and the creation of system trust. As Luhmann (1979) and Bachmann (2001) argue, both types of (interpersonal and 
system) trust go hand in hand as system trust needs to be enacted by repeated behaviours at the level of social interaction. Nonetheless, system trust, which is a form of institutionalised trust, also exists as a to some extent as separate behavioural pattern, strongly linked to the well-functional of accountability systems and democratic structures and can continue even when a small number of individuals lose trust in each other.

Thus, boundary-spanning roles, in particular those fulfilled by accountability agents, i.e. inspectors, are vital to provide a commonly acceptable basis of standards and expectation; and only where these are in place, it is possible to create an education system that is based on trust and has a strong potential to enhance its capacity and effectiveness in regard to its essential function within the wider society.

\section{Conclusion}

This chapter has investigated the role of accountability in ensuring a high level of performance outcome of education systems and their capacity for improvements. We explained how capacity and accountability interact in capacity being a precursor to effective accountability, while accountability can equally build capacity. Schools for example need the capacity to act on inspection feedback while capacity is required to inspect schools and hold schools accountable. Similarly, accountability systems, when offering feedback on improvement to schools, can lead to better learning outcomes.

Given the wide-spread evidence that accountability systems in some cases also fail to improve learning outcomes, we further explored how accountability constraints or supports system-wide improvement through their normalisation and coordination of standards of high-quality education. We argued for two mechanisms by which accountability systems can build capacity for improvement: alignment of actions of stakeholders across the system as well as their expectations of what good education constitutes and where improvements are needed. These mechanisms however only operate if, and when, the accountability system and those working in it (e.g. school inspectors) operate as boundary spanners in standardising and normalising practices of schools, parents, learners and those working with schools. Such a boundary spanning role is contingent on whether the accountability system is based on a set of transparent and reliable control procedures that schools and other stakeholders can engage with and incorporate in their work. And also whether schools and other relevant stakeholders view the accountability agents' actual practices as fair and legitimate and consider the accountability exercise to be in their best interest. We discussed a number of examples of how Inspectorates of Education gain such legitimacy through extensive and genuine consultation over their frameworks for inspection, and by engaging the teaching profession in their inspection activity. However, when an accountability exercise is riddled with deception, in transparent decision-making, blame games, hidden agendas or misuse of power on the side of the accountability 
agent, trust in the accountability system is clearly broken. Only if malpractices of this kind are absent it seems possible to reap the benefits of accountability and raise the improvement capacity and quality of education systems in a sustainable manner.

\section{Notes}

1 https://www.gov.uk/government/organisations/ofsted/about; https://www.ed.gov/essa.

2 New York State Testing Program (2018). NY Educator Guide to the 2018 Grades 3-8 Mathematics Tests.

3 https://onlinelibrary.wiley.com/doi/full/10.1002/berj.3264.

4 Taken from http://johnbraithwaite.com/responsive-regulation/ and https://www.anu. edu.au/fellows/jbraithwaite/_documents/Articles/essence_responsive_regulation.pdf.

5 https://www.bbc.co.uk/news/education-36188634.

6 https://www.gov.uk/government/consultations/education-inspection-framework2019-inspecting-the-substance-of-education/education-inspection-framework-2019inspecting-the-substance-of-education.

7 https://thirdspacelearning.com/blog/new-ofsted-framework-2019-consultationresults-schools/.

\section{References}

Altrichter, H. (2017). The short flourishing of an inspection system (pp. 207-230). In: J. Baxter (Ed.), School Inspectors. Dordrecht: Springer.

Allen, R., \& Burgess, S. (2012). How Should We Treat Under-Performing Schools? A Regression Discontinuity Analysis of School Inspections in England. Bristol: University of Bristol, Centre for Market and Public Organisation, Bristol Institute of Public Affairs.

Bachmann, R. (2001).Trust, power and control in trans-organizational relations. Organization Studies, 22(2), 337-365.

Bachmann, R., \& Inkpen, A. C. (2011). Understanding institutional-based trust building processes in inter-organizational relationships. Organization Studies, 32(2), 281-301.

Bachmann, R., \& Kroeger, F. (2017). Trust, power or money:What governs business relationships? International Sociology, 32(1), 3-20.

Beugelsdijk, S. (2005). A note on the theory and measurement of trust in explaining differences in economic growth. Cambridge Journal of Economics, 30(3), 371-387.

Braithwaite, J. (2011). The essence of responsive regulation. UBCL Rev., 44, 475.

Bubb, S., \& Earley, P. (2008). From Self-Evaluation to School Improvement: The Importance of Effective Staff Development. Reading: CfBT Education Trust.

Churches, R., \& McBride, C. (2013). Making External School Review Effective. Reading: CFBT.

Coleman, J. S. (1990). Foundations of Social Theory. Cambridge, MA: Harvard University Press.

Courtney, S. J. (2012). Ofsted's revised school inspection framework: Experiences and implications. In: Paper Presented at BERA Conference, Manchester. Available from http://www. leeds.ac.uk/educol/documents/216133.pdf.

Daly, A. (2009). Rigid response in an age of accountability: The potential of leadership and trust", Educational Administration Quarterly, 45(2), 168-216.

Darling-Hammond, L. (2004). Standards, accountability, and school reform. Teachers College Record, 106(6), 1047-1085.

Darvas, P., \& Balwanz, D. (2014). Basic Education beyond the Millennium Development Goals in Ghana: How Equity in Service Delivery Affects Educational and Learning Outcomes (No.: 978-1-4648-0098-6). Washington, DC: World Bank Studies. 
Dedering, K., \& Sowada, M. G. (2017). Changing policies — Changing inspection practices? Or the other way round? (pp. 25-43). In: J. Baxter (Ed.), School Inspectors. Dordrecht: Springer.

De Grauwe, A. (2001). School Supervision in Four African Countries (Vol. I), Challenges and Reforms. Paris France: International Institute for Educational Planning/UNESCO.

De Grauwe, A. (2007). Transforming school supervision into a tool for quality improvement. International Review of Education, 53(5/6), 709-714.

De Grauwe, A. (2008). School Monitoring Systems and their Impact on Disparities. Paris: UNESCO IIEP.

Dembélé, M., \& Oviawe, J. (2007). Introduction: Quality education in Africa - International commitments, local challenges and responses. International Review of Education, 53(5/6), 473-483.

Eddy-Spicer, D., Ehren, M., Bangpan, M., Khatwa, M., Perrone, F. (2016). Under What Conditions do Inspection, Monitoring and Assessment Improve System Efficiency, Service Delivery and Learning Outcomes for the Poorest and Most Marginalised? A Realist Synthesis of School Accountability in Low- and Middle-Income Countries. London: EPPI-Centre, Social Science Research Unit, UCL Institute of Education, University College London.

Ehren, M. C. M. (Eds.) (2016). Methods and Modalities of Effective School Inspections. Dordrecht: Springer.

Ehren, M. C. M., Altrichter, H., McNamara, G., \& O’Hara, J. (2013). Impact of school inspections on school improvement; Describing assumptions on causal mechanisms in six European countries. Educational Assessment, Evaluation and Accountability, 25(1), 3-43.

Ehren, M. C. M., \& Hatch, T. (2013). Responses of schools to accountability systems using multiple measures:The case of New York City elementary schools. Educational Assessment, Evaluation and Accountability, 25(4), 341-373.

Ehren, M. C., \& Shackleton, N. (2016). Mechanisms of change in Dutch inspected schools: comparing schools in different inspection treatments. British Journal of Educational Studies, 64(2), 185-213.

Ehren, M. C. M., Gustafsson, J. E., Altrichter, H., Skedsmo, G., Kemethofer, D., Hüber, S. (2015). Comparing effects and side effects of different school inspection systems across Europe. Comparative Education, 51(3), 375-400.

Ehren, M. C. M.,Visscher,A. J. (2006). Towards a theory on the impact of school inspections. British Journal of Educational Studies, 54(1), 51-72.

Ehren, M. C. M., Eddy-Spicer, D., Bangpan, M., Reid, A. (2017). School inspections in lowand middle-income countries: Explaining impact and mechanisms of impact. Compare, 47(4), 468-482.

Englert, K. S., Fries, D. A., Martin-Glenn, M. L., \& Douglas, B. B. (2007). Accountability systems: A comparative analysis of superintendent, principal, and teacher perceptions. International Journal of Education Policy and Leadership, 2(4), 1-12.

Fledderus, J., Brandsen, T., \& Honingh, M. (2014). Restoring trust through the coproduction of public services: A theoretical elaboration. Public Management Review, 16(3), 424-443.

Forsyth, P. B., Adams, C. M., \& Hoy, W. K. (2011). Collective Trust: Why Schools Can't Improve without It. New York: Teachers College Press.

Gorard, Stephen. (2009) "Does the Index of Segregation Matter? The Composition of Secondary Schools in England since 1996." British Educational Research Journal 35.4 639-52.

Grek, S. (2009). Governing by numbers: The PISA 'effect' in Europe. Journal of Education Policy, 24(1), 23-37.

Grek, S., \& Ozga, J. (2010). Governing education through data: Scotland, England and the European education policy space. British Educational Research Journal, 36, 937-952. 
Gunningham, N., \& Sinclair, D. (2009b). Regulation and the role of trust: Reflections from the mining industry. Journal of Law and Society, 36(2), 167-194.

Gunningham, N., \& Sinclair, D. (2009a). Organizational trust and the limits of managementbased regulation. Law \& Society Review, 43(4), 865-900.

Hall, C., \& Noyes, A. (2007). The impact of school self evaluation processes on British teachers' views of their work and professionalism. In: Paper Presented at the BERA, London. Available from http://www.leeds.ac.uk/educol/documents/167834.doc.

Harris, D.N. (2011). Value-added measures in education. Boston: Harvard Education Press.

Hussain, I. (2012). Subjective Performance in the Public Sector: Evidence from School Inspections. London: School of Economics and Political Science, Centre for Economic Performance.

Hutchinson, J. (2016). School Inspection in England: Is there room to improve?: London: EPI.

Jackson, J., Bradford, B., Hough, M., Myhill, A., Quinton, P., \& Tyler, T. R. (2012). Why do people comply with the law? Legitimacy and the influence of legal institutions. British Journal of Criminology, 52(6), 1051-1071.

Jaffer, K. (2010). School inspection and supervision in Pakistan: Approaches and issues. Prospects, 40(3), 375-392.

Luginbuhl, R., Webbink, D., \& De Wolf, I. (2009). Do inspections improve primary school performance? Educational Evaluation and Policy Analysis, 31(3), 221-237.

Koretz, D. M., McCaffrey, D. F., \& Hamilton, L. S. (2001). Towards a framework for validating gains under high-stakes conditions. CSE Technical Report 55. CRESST/Harvard Graduate School of Education.

Lasky, S., Schaffer, E. C., \& Hopkins, T. (2008). Learning to think and talk from evidence: Developing system-wide capacity for learning conversations. In: L. Earl \& H. Timperley (Eds.), Professional Learning Conversations: Challenges in Using Evidence for Improvement. The Netherlands: Springer.

Leckie, G., \& Goldstein, H. (2017). The evolution of school league tables in England 19922016: 'Contextual value-added', 'expected progress' and 'progress 8'. British Educational Research Journal, 43(2), 193-212.

Levi, M. (2003). Chapter 4. A state of trust. In: V. Braithwaite \& M. Levi (Eds.), Trust and Governance. New York: Russell Sage Foundation.

Linn, R. L. (2005). Conflicting demands of No Child Left Behind and state systems: Mixed messages about school performance. Education Policy Analysis Archives, 13(33), 1-17.

Looney, J. (2009). Assessment and innovation in education. OECD Education Working Paper No. 24 (EDU/WKP(2009)3).

Looney, J. (2011). Developing High-Quality Teachers: teacher evaluation for improvement. European Journal of Education, 46(4), 440-455.

Luhmann, N. (1979). Trust and Power. Chichester: Wiley.

MacBeath, J. (2004). Putting the self back into self-evaluation. Improving Schools, 7(1), 87-91.

Mansbridge, J. (2014). The role of the state in governing the commons. Environmental Science E Policy, 36, 8-10.

Marsh,J.A., \& Farrell, C. C. (2015). How leaders can support teachers with data-driven decision making: A framework for understanding capacity building. Educational Management Administration \& Leadership, 43(2), 269-289.

Matthews, P., \& Sammons, P. (2004). Improvement through Inspection. London: Ofsted.

Mayer, R. C., Davis, J. H., \& Schoorman, F. D. (1995). An integrative model of organizational trust. Academy of Management Review, 20(3), 709-734.

Mazibuko, S. P. (2009). The Managerial Role of the Principal in Whole-School Evaluation in the Context of Disadvantaged Schools in Kwazulu-Natal. 
Millward, P., \& Timperley, H. (2010). Organizational learning facilitated by instructional leadership, tight coupling and boundary spanning practices. Journal of Educational Change, 11(2), 139-155.

McEvily, B., Perrone, V., \& Zaheer, A. (2003). Trust as an organizing principle. Organization Science, 14(1), 91-103.

McNamara, G., \& O'Hara, J. (2012). From looking at our schools (LAOS) to whole school evaluation-management, leadership and learning (WSE-MLL): The evolution of inspection in Irish schools over the past decade. Educational Assessment, Evaluation and Accountability, 24(2), 79-97.

Pallas, A. M., \& Jennings, J. L. (2009). 'Progress' reports (pp. 99-105). In: Ravitch, D., Meier, D., Avitia, D., Bloomfield, D. C., Brennan, J. F., Dukes, H. N., Haimson, L., Horowitz, E. M., Jennings, J. L., Koss, S., McAdoo, M., Ofer, U., Pallas, A. M., Sanders, S., Stern, S. Sulivan, P. J., Wolf, A. (Eds.), NYC Schools Under Bloomberg and Klein:What Parents, Teachers and Policymakers Need to Know. New York: Cass Size Matters.

Penninckx, M., \& Vanhoof, J. (2015). Insights gained by schools and emotional consequences of school inspections. A review of evidence. School Leadership \& Management, $35(5), 477-501$.

Plowright, D. (2007). Self-evaluation and Ofsted inspection developing an integrative model of school improvement. Educational Management Administration E Leadership, 35(3), 373-393.

Rosenthal, L. (2004). Do school inspections improve school quality? Ofsted inspections and school examination results in the UK. Economics of Education Review, 23(2), 143-151.

Rothstein, B. (2011). The Quality of Government: Corruption, Social Trust, and Inequality in International Perspective. Chicago: University of Chicago Press.

Rothstein, B. (2013). Corruption and social trust: Why the fish rots from the head down. Social Research, 80(4), 1009-1032.

Shaw, I, Newton, D. P., Aitkin, M., \& Darnell, R. (2003). Do OFSTED inspections of secondary education make a difference to GCSE results? British Educational Research Journal, 29(1), 63-76.

Simons, H. (2013). Enhancing the quality of education through school self- evaluation In: M. K. Lai \& S. Kushner (Eds.), A Developmental and Negotiated Approach to School Self-Evaluation. Advances in Program Evaluation (Vol. 14). Bingley, UK: Emerald Group Publishing.

Schneider, J., Jacobsen, R., White, R., \& Gehlbach, H. (2017). The (Mis)Measure of Schools: How Data Affect Stakeholder Knowledge and Perceptions of Quality.

Yeager, D. S., Purdie-Vaughns,V., Garcia, J., Pebley, P., \& Cohen, G. L. (2012). Lifting a barrier of mistrust: "Wise" feedback to racial minorities. Unpublished Manuscript. Austin, TX: University of Texas. 


\section{6}

\section{INNER GROUP TRUST AND SCHOOL AUTONOMY IN A SEGREGATED SCHOOL SYSTEM; PARENTAL SELF-SEGREGATION IN THE NETHERLANDS}

\section{Melanie Ehren}

\section{Introduction}

The segregation of students into different schools has important implications for educational inequality, social cohesion and intergenerational mobility and has attracted much attention among social and educational academia and policymakers alike. Rising levels of segregation are observed across the globe as Parker et al. (2018), Jerrim et al. (2018) and Gutiérrez et al. (2017) show. These authors all present OECD PISA data which illustrates how the segregation of rich and poor pupils has remained broadly unchanged across OECD countries and is even increasing in some countries, despite numerous policy initiatives designed to reduce socio-economic gaps.

The Netherlands is one of the countries that has seen an increase in between-school segregation. In its most recent annual report of the State of Dutch Education, the Inspectorate of Education (2018) presents a concerning narrative of how schools are increasingly segregated along ethnic lines and educational background of parents. Segregation on both dimensions (ethnic and educational background of parents) is particularly high in the four large cities in the Netherlands (The Hague, Amsterdam, Rotterdam and Utrecht), but even schools in some smaller cities, such as Ede and Breda, are highly segregated (Boterman, 2019; Inspectorate of Education, 2018; Ladd et al., 2009). Segregation of children with different migratory backgrounds seems to be in decline in most cities while level of parental income is becoming more prevalent in how students are sorted into different schools, according to Boterman (2019).

A high level of segregation is an important academic and political concern as it is widely associated with the reproduction of inequalities across class, race and gender (Sampson, 2012). Previous research has suggested that having a high 
proportion of students from disadvantaged backgrounds as one's peers has a negative effect on a range of educational outcomes (Gutiérrez et al., 2017). Schooling systems which tend to cluster low socio-economic status students together could be increasing educational inequality and reducing social mobility over time (Levacic and Woods, 2002). Jerrim et al. (2018) reference OECD research which finds that in countries where schools tend to be more segregated, the impact of the school's socio-economic intake on learning outcomes is higher. This means that schools which serve disproportionate numbers of disadvantaged students are less able to counter the effects of that disadvantage than schools with a more balanced, comprehensive intake.

The effects of social segregation between schools are not limited, however, to student achievement alone. According to Gutiérrez et al. (2017) prior research has also found that greater levels of between-school segregation have an effect on school attendance, grade retention and behaviour (Palardy, 2013; Palardy et al., 2015). Altonji and Mansfield (in: Duncan and Murnane, 2011) describe how a concentration of students from deprived backgrounds creates a context which inhibits effective teaching and learning: a child from a poor family is two to four times as likely as a child from an affluent family to have classmates with low skills and with behaviour problems; this has a negative effect on the learning of the entire group as teachers tend to have lower expectations of what children can achieve and spend large amounts of time on managing behaviour instead of instructing students (Gilsing and Tierolf, 2010). Urban families living in poverty also tend to move frequently, and this further disrupts teaching and learning. A substantial body of research shows that schools serving high concentrations of poor, non-white and low-achieving students find it difficult to attract and retain skilled teachers (Duncan and Murnane, 2011, p. 14).

The extent of between-school segregation in an education system, therefore, matters, with some believing that encouraging greater mixing of young people from different social backgrounds is the key to reducing educational inequalities. Indeed, some scholars have even argued that socio-economically segregated schools fail to prepare students for facing diversity (Masey and Fisher, 2006) and may even be a threat to social cohesion (Gorard, 2009; Mickelson and Nkomo, 2012). As Gutiérrez et al. (2017) explain, the extent to which social groups mix is thought to be an important factor influencing inequality, social cohesion and social mobility (Gorard, 2009; Levacic and Woods, 2002). As long-lasting friendships and peer groups are developed during young people's time in school, the extent of between-school segregation is a key indicator of whether different social groups live in isolation from one another.

High levels of segregation are particularly problematic in the current time where nationalism is on the rise and a lack of opportunities is sparking an increase in populism. Segregating children from an early age is seen to contribute to ever more divided societies in which people who have different codes, 
values and belief systems find little common ground. Dividing children from a very early age provides them with little opportunity to socialize with and come to understand peers from different backgrounds, and this will have bearing on how they see and interact with others later in life. Gamoran $\left(2013^{1}\right)$, for example, talks about the socially divisive nature of school segregation when schooling is no longer a common socializing experience to instil a set of shared values and allow children to forge bonds with children from other backgrounds and origins. Diverse schools are better able to create social networks that cross the boundaries of families and communities and create networks that help knit the fabric of societies, according to Gamoran (2013).

The divisive nature of school segregation is acknowledged by national government in the Netherlands and several large municipalities (Amsterdam, Utrecht) who have introduced centralized systems for school intake to ensure more diverse schools. Nijmegen, a city in the south of the Netherlands, for example, introduced a central subscription system in 2009 to assign students to primary schools, ensuring a share of 30 per cent of disadvantaged students in each school. All the primary schools in Nijmegen have subscribed to the system: in the event of oversubscription, priority is given to siblings and children who live nearby but subsequent priority is given to either advantaged or disadvantaged students in order to reach the required balance, by lottery system (Jerrim et al., 2018).

On a national level, the most constant policy instrument has been the extra funding of schools based on school composition. Scheerens et al. (2019) describe how the Dutch Ministry of Education's funding formula prioritizes low socio-economic status (SES) and cultural minority students in an attempt to make these schools more attractive, such as when schools use the extra funding to reduce class size or offer additional support. Since 2010, schools in areas of high deprivation also receive further financial support to educate their disadvantaged students.

Despite these policies, school segregation remains high, particularly by level of income in the large cities (Boterman, 2019). This chapter looks at the regulatory context of freedom of education and school choice and how these provide the institutional context which enables and aggravates between-school segregation. The chapter focuses on primary education as a discussion of the mechanisms of segregation in secondary education would require a consideration of other structural conditions which separate students across schools, and particularly the highly tracked system of secondary education in the Netherlands. The chapter frames the discussion of school choice mechanisms around inner group trust and class closure and how this is causing parents to self-segregate their children into homogenous primary schools. We examine the high general trust in the Netherlands and the high trust in the education system and why these are not enabling a more diverse system. Our chapter ends with a discussion of potential policies to address the potential divisive nature of a segregated system, within a context of high school autonomy and school choice. 


\section{The Dutch education system and institutional context of segregation}

A number of studies address the levels, causes and effects of school segregation in the Netherlands, describing the type of segregation (e.g. according to ethnicity, level of income and parental education), geographical trends and how these interact with parental school choice and school profile and quality (Dijkstra et al., 2002; Gramberg, 1998; Karsten et al., 2003, 2006; Ladd et al., 2009). An understanding of such patterns of segregation, however, requires a brief introduction of the Dutch education system, and particularly the freedom of education and school choice.

\section{The Dutch education system}

Primary education in the Netherlands consists of 8 years of education, from the age of 4 until the age of 12 (grade 1-grade 8). The Dutch constitution (Article 23) guarantees the freedom of school choice, as well as the right for anyone to set up faith schools or schools with a specific pedagogical concept, which can be a public or a (semi) private school. Public schools are open to all children regardless of religion or world view and are generally subject to public law. They provide education on behalf of the state and are governed by the municipal council (or a governing committee), a public legal entity or a foundation set up by the council. Privately run schools are governed by the board of the founding association (e.g. a church or parents' association); they can refuse admittance to pupils whose parents do not subscribe to the pedagogical concept, belief or ideology of the school. These schools are also government funded, even though they have not been founded by the state. Teaching is based on religious or ideological beliefs, and this category includes Catholic, Protestant, Jewish, Muslim, Hindu and anthroposophical schools.

Freedom of education gives the right to any natural or legal person to set up a school, to organize teaching and to determine the (educational, religious or ideological) principles on which teaching is based. This constitutional arrangement

puts public and private schools on an equal footing, with all schools receiving public funding provided that they comply to legislator requirements. Parents have free school choice, and funding 'follows the student', which lays the foundation for potentially strong competition among schools (Nusche et al., 2014, pp. 20-21).

The Ministry of Education, Culture and Science has the responsibility for the overall education system, but it does not interfere with the organization of individual schools. The Ministry's responsibilities relate mainly to setting legislation and determining the structure and funding mechanisms of the education system. It also controls the system by setting quantitative or qualitative standards, attainment targets and examinations. The Minister of Education is responsible for the evaluation of the quality of education, which is carried out by the Dutch 
Inspectorate of Education (Scheerens et al., in: Volante et al., 2019). Scheerens et al. (2019, p. 115) describe the role of central government in education reform as 'establishing the objectives of the policy measures (what) while the field itself will decide how best to pursue those objectives (how)'.

The constitutional right of freedom of education has led to a diverse landscape of schools with Catholic (30\%) and Protestant (30\%) schools, a small number of Islamic and Hindu schools, and large number of schools that teach according to the methods of Maria Montessori, Helen Parkhurst (Dalton Plan schools) or anthroposophical philosophy (Steiner/Waldorff schools) (Boterman, 2019). Particularly the larger urban areas would have all or most types of schools, whereas Catholic schools are predominantly located in the Southern parts of the Netherlands, and a particular sub-fraction of Protestant schools or non-religious maintained schools are concentrated in the heartland of the country, reflecting the religious and socio-cultural profile of the local population. The diversity in offer of schools allows parents to purposefully decide on educating their child within a specific instructional approach or religious identity. All schools, however, have to teach towards the same academic outcomes and are held accountable on the same inspection standards.

\section{A self-segregated landscape}

School choice dynamics in the Netherlands are responsible for a substantial share of segregation, according to Boterman (2019), but school choice is to a large degree also informed by residential processes and where families live. As parents, particularly in primary education, favour the school that is closest to their home, spatial patterns of where ethnic groups and people from various social classes live will affect the student's population in schools. A good understanding of active self-segregation by school choice, thus, requires us to isolate school segregation from residential segregation.

Gutiérrez et al. (2017) discuss the variety of indices which have been developed to measure the segregation of individuals across different groups of schools. These indices differ, according to these authors in terms of their statistical properties as well as whether they attempt to measure segregation between just two or multiple groups. For instance, Massey and Denton (1988) classified indices of residential segregation according to five different dimensions: evenness, exposure, concentration, centralization and clustering. In the school-segregation literature, measures usually incorporate 'evenness' and 'exposure'. Evenness refers to differences in the distribution of two social groups among schools in a country. A school system is even if the allocation of students to schools matches their overall proportion at a national level. A school system is uneven if the proportion of students within one or both groups at schools greatly differs from their national proportion.

Exposure refers to the degree of potential contact, or the possibility of interaction, between two different groups within schools in a country. The probability of interaction between groups is given by the proportion of individuals per school 
who are part of each group. A very segregated school shows low exposure, as there are very few students from other groups than the majority group. Examples of indicators measuring exposure are the interaction or the isolation index (II).

Similar indices have been calculated by Boterman (2018, p. 163) for the Netherlands. His three segregation indices capture the segregation of a particular geographical area based on the dispersion of pupils across schools, while also taking into account the size of various groups within each municipality. These three indices allow us to understand where parents are actively choosing a school outside of their residential area:

- Index of dissimilarity (DI): the unevenness of the distribution of pupils over schools between two groups. It is not sensitive to the sizes of the groups and is, hence, useful for inter-urban comparisons.

- II: expresses the degree to which a particular group is concentrated in schools with a large share of their own group. It basically measures the average share of pupils of that same group at any particular school within the municipality. This index is highly sensitive to the total share of that group within the city. The likelihood of being in a school with, say Turkish-Dutch pupils, is higher if the share of Turkish pupils in the total school age population is higher.

- $\quad$ Modified II (MII): takes the share of pupils into account and, therefore, does a similar job as the DI but without comparing it to another group.

Boterman (2018) finds that The Hague, Ede and Breda score particularly high on ethnic segregation; Turkish-Dutch and Moroccan-Dutch children are concentrated in the same school in these cities, while Surinamese Dutch are particularly segregated from native Dutch children in Ede, the Hague and Amsterdam. The actual size of each of the ethnic groups in these cities is an important explanation for why the various groups are concentrated within a school and the effect largely disappears when correcting for group size, apart from the cities of Ede and Zwolle and for Surinamese-Dutch children. When calculating these measures for groups with various educational levels, Boterman finds that the gentrification of larger cities (Amsterdam/Utrecht) and the influx of higher educated parents is changing the landscape of school segregation. Amsterdam and Utrecht are becoming less segregated, when correcting measures for the growing size of high versus low educated groups, while segregation according to educational background is increasing in Almere, Haarlemmermeer and The Hague. Regardless of the size of the groups, the actual levels of segregation according to social class are very high (Boterman, 2018, Inspectorate of Education, 2018). In effect, children from Turkish, Moroccan and Surinamese descent do not go to school with native Dutch children in most of the cities and towns in the Netherlands, while children from low and high socio-economic backgrounds also concentrate in homogenous schools.

Parents carefully navigate the educational landscape and often choose a school outside of their residential area. The more options they have available, the greater 
the potential to choose a school that fits their choice pattern and preferences. How can we understand segregation from the various school choice sets of parents?

\section{School choice and parental preferences}

Most of the international literature concentrates on the school choice of middle-class parents who navigate the schooling landscape to ensure their child enters the best school and has access to a wide range of educational opportunities. The strategies they use range from moving into desired catchment areas for public schools, or various opt-out routes, such as buying into private schools or after school tuition (Bell, 2005; Burgess et al., 2009). Free school choice in the Netherlands does not require parents to move house to gain access to a favourable school; they sometimes, however, have to travel further and purposefully choose to do so. Particularly families in urban and suburban settings have access to a range of nearby schools and such access is even greater for wealthy parents who have more options for transporting their children to a school of their choice. Options for school choice, thus, vary widely, but are particularly exercised by middle and upper class parents and in urban areas.

Boterman (2019) shows how, in such areas, parents' often actively choose a school outside of their residential area and how this is increasingly leading to a landscape of homogenous schools with a student population that is similar in ethnic, income and parental educational background. In such contexts, school choice is not just informed by spatial proximity but also by (1) a school's religious or pedagogical profile, and (2) the (perceived) school quality and composition of the school population (Boterman, 2019; Dijkstra et al., 2002; Gramberg, 1998; Karsten et al., 2003, 2006; Ladd et al., 2009).

\section{Religious and pedagogical profile}

A report by Zeldenrijk (2011) explains how parents' choice of a school with a specific religious or pedagogical profile is motivated by the school population rather than actual differences in religious character or teaching ideology. These profiles signal a certain ethnic or socio-cultural student population and, according to Zeldenrijk (2011) schools do not vary much in how they teach or interact with children and parents. Small religious schools (Islam, Hindu and Jewish) tend to have students from the same ethnic background where Turkish/ Moroccan-Dutch parents favour an Islamic school, while native Dutch upper class parents seem to prefer Montessori, Steiner or Jenaplan schools (Inspectorate of Education, 2018).

\section{Perceived school quality and composition of the school population}

Perceived school quality in the Netherlands is a second key element of school choice dynamics, according to Boterman (2019). School quality in the 
Netherlands is measured by the Inspectorate of Education who assess schools on a set of indicators on the quality of teaching in schools, the academic outcomes of students on standardized assessments. The Inspectorate visits all school boards and a sample of their schools on a regular basis, prioritizing school boards with a relatively high number of underperforming schools. Inspection outcomes of all schools and their school boards are published on the internet. These inspection reports are expected to inform school improvement but also allow parents to choose a school of high quality. School inspection reports are assumed to inform parents' decisions-making, and various initiatives have been implemented over the past decade to make the reports more accessible, such as using 'lay language' to explain the performance of schools on the inspection standards, using colour coded visuals to present the overall judgement of school quality and publishing separate lists of weak schools.

Studies by Dronkers (1999) and Dronkers and Veenstra (2001), however, suggest that parents rarely use inspection reports when deciding on a school; according to these authors, only $2 \%$ of the variance in the growth of schools can be explained by the publication of school performance data, and only $20 \%$ of middle and upper class parents seem to take the inspection judgement of a school and its outcome on national standardized tests into consideration (Karsten et al., 2010; Gilsing and Tierolf, 2010). A more recent study by Boterman (2013) suggests that parents associate school quality with the composition of the school instead of the inspection measure of quality; they would talk about quality when being asked to explain their choice of schools, but further probing indicated that their actual choice was informed by the student population in the school.

This lack of parental preference for schools with high learning outcomes or quality teaching concurs with international studies. Bell's (2005) study on why parents in the United States choose a middle or high school that was classified as 'failing' shows a complex dynamic of 'choice sets' whereby parents take a variety of factors into account which vary according to their own background and the availability of schools within travelling distance of their home. Using social networks, customary attendance patterns and their understanding of their child's academic achievement, parents construct a set of preferences for types of schools that vary systematically by social-class background. These preferences generally include criteria other than the academic outcomes of the school.

In the Netherlands, Karsten et al. (2003) refer to 'a match between home and school', while Boterman (2013) quotes parents who talk about the atmosphere in the school. Such information is typically obtained through 'word of mouth' or other informal communication among parents, according to Karsten et al., 2010; Gilsing and Tierolf, 2010). Parents seem to prefer a school profile which matches their own educational experience, and a school population that is similar to their own background.

For some parents in the Netherlands, such choice is a negative decision. Karsten et al. (2010) and Boterman (2019) refer to a 'white flight' in some areas where parents purposefully avoid schools with a predominantly migrant 
population and instead send their child to relatively homogenous 'white schools', even if they have to travel out of their own residential area. Various studies have presented examples of white native parents who are anxious about 'black schools' where parents speak Moroccan at the school gate. Similarly, lower class parents are often uneasy about mixing with upper class parents who come and collect their children by expensive 'cargo bikes'; electric bicycles with a cargo box in front to carry one or two children to school. The small number of faithbased schools also lead to high segregation when Muslim, Hindu and orthodox Protestant families send their children to these schools; motivations to do so often go beyond the specific religious profile of the school when Muslim parents, for example, do not want their child to mix with peers who are seen to have looser sexual and ethical values (Zeldenrijk, 2010). There are examples of parents deliberately choosing schools with a diverse student population (Reay et al., 2011), but more often parents self-segregate into homogenous schools. It is these understandings of how their child will be exposed to other socio-cultural and/or religious values and use of language in the school community which dominates parents' assessment of school quality and school choice.

\section{Self-segregation: A reinforcing mechanism}

Self-segregation seems to have a reinforcing element, particularly in areas where there are multiple schools to choose from: the more homogenous a school becomes, the less likely it will attract parents and children from other ethnic, cultural or socio-economic groups. Parents purposefully avoid schools when the majority of children have a different ethnic or socio-economic background (Gilsing and Tierolf, 2010). Boterman (2018) explains this reinforcing loop by the fear of being stereotyped or bullied when being the only white, Muslim or orthodox Protestant child in the group. In primary education, parents also meet other parents at the school gate when collecting their child from school and will wish to congregate with like-minded parents for reasons of cultural, social and economic belonging (language, religion, family, friends, etc.). Native Dutch parents often express an anxiety about a potential drag on the language development of their child when going to school with non-native speakers or their child being exposed to strict gender/sexual values and norms they do not agree with. In some cities, ethnic groups are large in numbers and concentrate in certain residential areas; they can easily uphold their own language and culture, motivating native Dutch parents to move out of the area and travel to a school outside of the neighbourhood. Zeldenrijk (2010) refers to 'class closure' when highly educated parents prefer a school with children from a similar socio-economic background, and lower educated parents avoid schools with children from a different ethnic background (see also Gilsing and Tierolf, 2010; Jongejan and Thijs, 2010). The stronger the identity of the majority group, the more homogenous a school will become, particularly when parents stereotype schools in the neighbourhood according to 'black', 'white', 'workers' or 'posh', and where each of 
these stereotypes has a specific set of constructs and represents a certain 'habitus' or social and cultural capital.

Forsyth et al. (2011) explains how groups vary in terms of the kinds and degree of diversity found within them and the extent to which they can be characterized by a shared identity, or psychological disposition which generates similar kinds of behaviours and preferences (Collier, 2017). People will feel a sense of belonging to a group where other members share similar attributes or behaviours. A group of parents in a school are typically similar in the clothes they wear, the language and accent they speak, whether they have body tattoos, their means of transport, the type of food their children eat at lunchtime, how their children spend their leisure time and what kind of holidays are celebrated. The more homogenous the school population is in terms of having a shared culture; a set of shared social norms; or similar somatic characteristics (hair, skin and colour), genetic origin, linguistic profile, cultural roots, upbringing and religion, the stronger its group identity. It is most likely these features parents will pick up on when visiting opening evenings or touring the school grounds.

In some cases, school policy is reinforcing the construction of these identities and the pattern of self-segregation, such as when schools set high (voluntary) fees for extra-curricular activities, offer programmes of study which particularly cater for the interests of middle and upper class parents (e.g. dual language curriculum), present themselves as a homogenous school or reject applications on the basis of the school's religious profile (Gilsing and Tierolf, 2010). By signalling a certain profile, they motivate groups of parents to subscribe their children to the school, while dissuading others from doing so. Schools that are oversubscribed will often also select their intake and try to prioritize enrolment of potentially high performing pupils from middle and upper class backgrounds. Self-segregation becomes a reinforcing loop whenever more homogenous schools become less popular to parents with different religious beliefs or pedagogical preferences, or when schools with a predominantly disadvantaged intake become specialized in teaching these children and become less attractive for parents from more affluent backgrounds (Gilsing and Tierolf, 2010).

\section{School choice and trust in the education system}

The previous sections highlighted how parents in the Netherlands prefer schools which match their home environment and values and how the religious and pedagogical profile of the school and its student population are the primary sources of information by which they chose such a school (provided there is choice in their neighbourhood). Quality in terms of student outcomes or inspection judgement seems to matter less to parents. This section argues that the high general trust in the education system and the legitimacy of a highly segregated system, rooted in Article 23 of the Constitution, are central to understanding why school quality is not at the forefront of parental school choice and why strong group identity trumps general trust. 


\section{Trust in the education system}

In the Netherlands, trust in the education system is relatively high compared to other countries. In its 2015 'Government at a Glance' report, the OECD presents results from the Gallup World Poll, indicating that the Netherlands is one of the highest scoring countries with Belgium, Ireland and Finland in citizens' satisfaction with the education system. Satisfaction with the education system also increased from 67 to $78 \%$ of the population; the OECD average was 67\% in 2016 (see Figure 6.1). Data refer to the percentage of 'yes' answers to the question: 'In the city or area where you live, are you satisfied or dissatisfied with the educational system or the schools?'

The high trust in the education system suggests that parents expect all schools to be of (similar) good quality and may, as a result, think that it does not matter where a child goes to school in terms of learning outcomes or entry to secondary education. A recent survey of 'Ouders en Onderwijs' (an advocacy group for parents) supports this statement as only $5 \%$ of the parents report their child having experienced inequalities in educational opportunities.

The expectation that schools vary little in quality or children's academic outcomes is further reinforced by the Inspectorate's statutory framework and mission statement which emphasize its role in 'guaranteeing a minimum quality level across all schools'. All schools have to meet minimum outcome targets by legislation, ensuring that all children exit primary education with a similar level of academic outcomes. As identity, culture, ethos and values in the school are purposefully outside of the accountability and regulatory framework and protected by Article 23 of the Constitution, these are the factors that parents expect and observe to vary across schools. Their choice of a school with a highly homogenous population suggests that these identities are the main mechanism

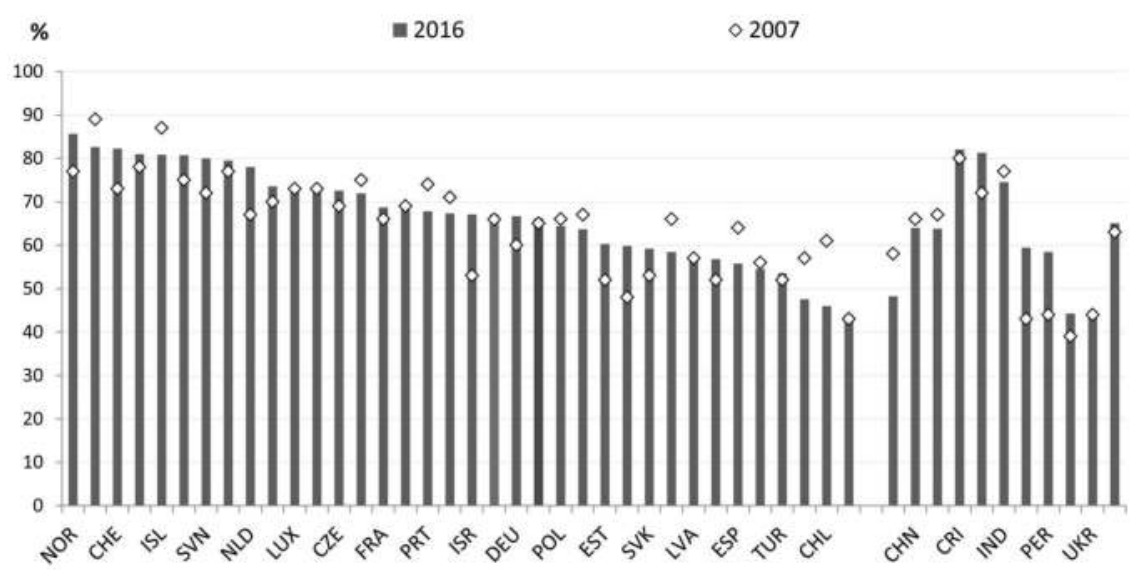

FIGURE 6.1 Citizens' satisfaction with the education system (2007 and 2016) Source: OECD Government at a Glance (2017). 
by which schools become segregated and are considered a legitimate element of, and preference for school choice.

\section{A pillarized society and Article 23 of the Constitution}

To understand the current legitimacy of the highly segregated school landscape and the role of Article 23 of the Constitution in maintaining such a divided system, we need to understand the historic context of the education system and society in general. Sturm et al. (1998) and Driessen and Van der Slik (2001) refer to the remarkable subcultural segmentation of education and of society in general on the basis of different religious or philosophical views and how this 'pillarized system' can be traced back to the 17th century but still shapes our current thinking of how to organize schools and school choice.

As Sturm et al. (1998) describe, from around 1880 to around 1970, public life in the Netherlands was primarily divided along ideological lines and the different pillars were harmoniously living apart in what was termed a 'consociational democracy'. These pillars not only represented various religious groups, but also the emerging social democratic labour movement. Each pillar had its own set of values, identities, outlook on life, media, art forms, youth organizations, old age homes, sport and cultural clubs, social interest organizations, housing associations, insurance companies, health institutions, etc., providing citizens with a more prominent and strong marker of their identity, often even more so than socio-economic, functional or regional forms of solidarity or, according to Sturm et al. (1998), even kinship. The state accommodated to the citizens' different ideologies by pluralizing the services it controlled as well as by incorporating the differing world views into the public order. State subsidies were allocated through the pillars according to the principle of proportionality. In this way, the presence of an intermediate layer of ideologically based private organizations, distributing public money for educational as well as social and cultural purposes, became a salient feature of the Dutch welfare state in the course of the 20th century. Pillarization was a cradle-to-grave pluralistic organization of society in which functional differentiation was overlaid by world view differences.

Each pillar, thus, had its own familiar and isolated culture, and education was an important part of the provision, particularly in educating a dedicated cadre with leadership abilities. All pillars maintained their own schools, apart from the social democrats who preferred religiously neutral public schools provided by the state. Only these schools were state funded at the time, but a long dispute over funding resulted in an agreement between liberals and socialists on the left and Christian democrats on the right in the Netherlands on equal funding of public and religious schools in 1917. This political agreement, termed the 'Pacification', ended both suffrage issues as well as dispute over unequal school funding. Article 23 of the Dutch constitution was introduced to separate school and the state by funding all schools equally, both public and private and 
maintaining freedom of education for all schools in, for example, curriculum policy and teacher appointments.

The pacification, however, resulted in even further pillarization of the education system with, according to Sturm et al. (1998), an enormous increase in the number of denominational schools of widely divergent kinds, at the cost of public schools. A few years after the implementation of Article 23, the number of denominational primary schools already outnumbered the neutral public ones, and this is still the case today. According to Driessen and Van der Slik (2001), in 1999-2000, 34\% of schools are public, 30\% are Protestant Christian, 30\% are Roman Catholic, while another 7\% of schools have one of 16 other denominations (e.g. Islamic, Hindu, Jena Plan and Montessori).

While the church has lost much of its influence over social institutions and organizations in the past decades, including schools, the segregation across past religious and pedagogical profiles has remained largely intact. In 1950, for example, $73 \%$ of all elementary school children attended a private school; in 1993, the number had only dropped to 68\% (Dronkers, 1996). Bax (1988) explains this continued segmentation of the education system by the vested interests of the professionals in denominational organizations and their legal protection. Even parts of the structure of education support and pedagogical counselling services continue to be organized according to denominational profiles.

Dronkers (1996) adds a few other possible explanations. He refers to the informal relations between school administration and teachers in private schools which may contribute to the high achievement of students in such schools and thereby attract nonreligious parents. Additionally, private schools may be more attractive than public schools because, in contrast to the obligation of the latter to be neutral, private schools pay considerable attention to basic values and norms. According to Dronkers, more traditional parents avoid public schools because they find them to be too progressive. Finally, parents and teachers tend to more purposefully choose a private school that meets their preferences and own background, and such purposeful choice is thought to encourage the development of an educational community with shared values and may thereby foster higher achievement. Private schools are, to some parents, thought to provide some counterweight to the negative influence of increased individualization in modern society. Furthermore, as Sturm et al. (1998) explain, the fact that the Netherlands is a small and very densely populated country allows a highly compartmentalized system to continue to exist and to increase when new minorities of immigrants utilize Article 23 to establish and access schools according to their own religious or cultural beliefs and identities, creating new segments of schools in society.

\section{But what about general trust ...}

The high self-segregation into homogenous schools is, however, remarkable given the high level of general trust in the Netherlands. Dutch citizens score generally high compared to other countries. The Eurostat (2015) survey indicates 


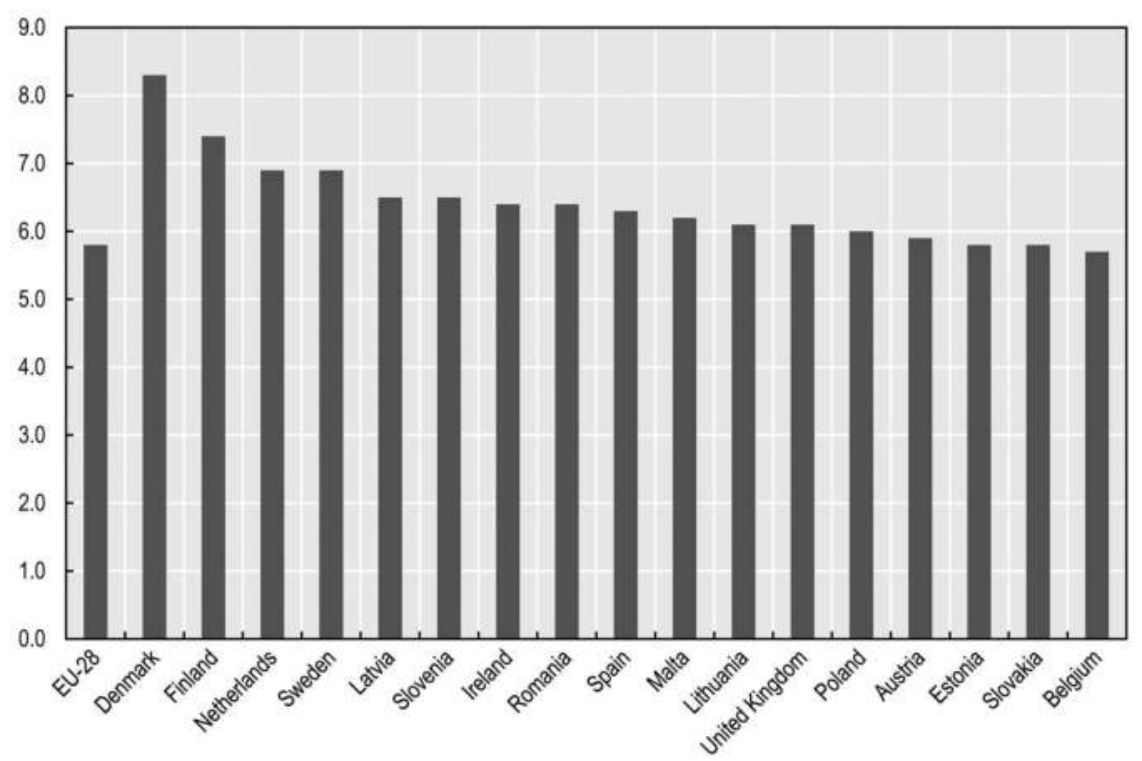

FIGURE 6.2 Interpersonal trust in European countries, $2013^{2}$

Source: OECD guidelines on measuring trust $\left(2017^{3}\right)$

a score of 6.9 compared to the average of 5.8 across 28 EU countries on the question 'Some people say that you can trust most people (see figure 6.2). Others think you cannot be too careful in dealing with other people. Do you think most people can be trusted?'.

General trust refers to 'the potential readiness of citizens to cooperate with each other and to abstract preparedness to engage in civic endeavours with each other. Attitudes of generalized trust extend beyond the boundaries of face-toface interaction and incorporate people who are not personally known' (Stolle, 2002, p. 397). Goodall (2015) and Morrone et al. (2009), for example, explain how, in such a context, others would not be seen as rivals, there would be a sense of a shared fate and common purpose and people would be inclined to trust others outside of their own group (Gunningham and Sinclair, 2009a; Morrone et al., 2009; Uslaner, 2015, p. 21).

Generalized trust arises, according to Rothstein (2011), from the institutional environment of laws, norms and standards on which people and organizations can rely. Having a clear set of rules and a well-functioning rule of law provides normative certainty and accountability of power, enabling people to enact their civil rights, while similarly enforcing duties and safeguarding autonomy. In societies with high generalized trust, people are more inclined to give others the benefit of the doubt; they have a more optimistic outlook for future interactions with people in general and are more engaged in public policy (Oomsels and Bouckaert, 2017; OECD, 2017). 
Given the high general trust of Dutch citizens, one would expect an education system of heterogeneous schools where race, income or culture are irrelevant conditions of school choice. A sense of shared purpose would allow parents to find common ground in their choice of schools, embrace diverse schools where division across ethnic and cultural background or income is overcome. We would also expect that general trust can only exist in a country where children learn to live with others from diverse backgrounds from a very early age and are motivated to do so by their parents who actively seek out diverse schools. This is evidently not the case as the previous sections of this chapter indicated.

Strong inner group identity, trust in the education system (including in the external accountability ensuring a high level of quality across all schools) and the legitimacy to choose on the basis of identity apparently trumps the general trust in creating a highly self-segregated school system. In the Netherlands, the institutional context of freedom of education and school choice simply does not require various ethnic and socio-economic groups to interact in an educational context and parents choose not to do so.

\section{Conclusion}

This chapter described the high levels of segregation in the Dutch education system where children from different ethnic backgrounds and levels of parental income are concentrated in homogenous schools. In the Netherlands, children from disadvantaged backgrounds have substantially less opportunities to succeed as they are increasingly advised to go into lower educational tracks in secondary education, whilst educated and affluent parents push for higher educational tracks for their children (Inspectorate of Education, 2018). This trend is unfortunately in line with other countries; a study by Gutiérrez et al. (2017) on OECD PISA data indicates that the level of between-school segregation is increasing in a number of OECD countries.

This is a striking and perhaps surprising finding, given how much the world has changed over the last decade. In particular, despite a host of school-system reforms occurring across the world and major world events such as the Great Recession of 2008, the segregation of students from different backgrounds into different schools has hardly altered at all. One explanation is the high residential segregation, but even when correcting for where parents live, parental choice of a schools outside of one's area is an important factor in between-school segregation in the Netherlands and elsewhere.

The high level of segregation in the Netherlands can be explained by looking at the institutional context of freedom of education and school choice which has resulted in highly homogenous schools, even in areas with a diverse population. A strong group identity, which is situated in and legitimized by a historic context of a 'pillarized' society and education system in the Netherlands has led parents to self-segregate into homogenous schools that matches their own schooling experience and background. A lack of societal consensus on the value of diverse 
schools and the protection of schools' identities in the constitution reinforces such patterns of self-segregation where mouth-to-mouth stereotyping of schools strengthens the emergence of homogenous schools, according to student population and identity.

The strong pattern of what Zeldenrijk (2011) calls 'class closure' is remarkable given the high general trust and high trust in the education system of Dutch citizens. High general trust is a measure of the potential readiness of citizens to cooperate with each other and to abstract preparedness to engage in civic endeavours with each other. Attitudes of generalized trust extend beyond the boundaries of face-to-face interaction and incorporate people who are not personally known. Such high general trust would suggest that parents either support diverse schools or at least do not actively seek out homogenous schools. However, in the Netherlands the freedom of school choice in a context of school autonomy has clearly not informed parents' choice for more diverse schools or created a national understanding or set of values to support more diverse schools. The fact that there traditionally is a strong structure to safeguard minority's interests via freedom of education seems to have implied that there was no need to actually trust or interact with unknown others.

This chapter discussed the various academic and political concerns over a highly segregated school system, arguing that segregation deprives children from opportunities to do well in school and progress in life and in fracturing society into groups that no longer coexist peacefully. Various authors discuss the potentially socially divisive nature of segregating students in schools as such systems do not offer a setting in which students from various groups interact or collaborate with each other and have limited opportunity to get to know others and develop trust towards other groups. When we need to engage with others in a pluralist society, in a time of limited resources, or when social cohesion is needed to grow the economy in more sustainable ways, our education system is not equipped to learn children how to do so. Students from different groups simply do not have opportunities to develop intergroup trust, and this will likely impact on the extent to which they will rely on others from other backgrounds later in life. Volante et al. (2019) argue that high between-school segregation is particularly problematic given the current increase in nationalism and increased and hardened political divisions emerging within countries across the world. This begs the question of how we can come to a system with more diverse schools, given the high levels of school autonomy, freedom of education and school choice in the Netherlands.

Volante et al. (2019) explain how, internationally, most of the policies designed to reduce socio-economic inequalities among students fall within one of the following three dimensions: (1) school autonomy versus centralization, (2) tracking versus comprehensive schooling and (3) instruction time and curricula. Policies they describe include the introduction of more inclusive curricula, reducing tracking and streaming by ability level, or more centralized targets on student performance. In the Netherlands, policies have traditionally included 
preferential funding to schools in deprived areas and/or with students of low SES and migrant backgrounds. Other, more recent initiatives are the introduction of citizenship education. The Education Act was amended in 2005 to task schools with the 'promotion of active citizenship' and aimed to ensure that children learn to interact with peers from other backgrounds and cultures and value the pluralist nature of Dutch society from an early age on. Further changes were announced in 2019 which included legislating for a minimum standard of skills and competences children need to acquire by the end of compulsory education. These standards require students to understand and know the principles of civic society, democracy and the rule of law, while they are also expected to have the skills to live and communicate with others in a pluralist society. Various assessments are currently being developed to allow schools to evaluate children's civic competences (e.g. Cito meetinstrument burgerschapscompetenties), while the Inspectorate of Education will incorporate these standards in their framework.

The change in legislation can be seen as a first attempt to create a set of shared values and an identity which would connect the various groups in society. Schools, however, continue to be relatively autonomous in deciding on how to meet these goals; they are only required to publicly report on how they have incorporated these aims in their curriculum and assessment. The current inspection framework also only holds schools accountable for the performance of their own student population and as such provides little incentives for a more integrated system. Given the freedom of education and school choice, schools only need to offer a structured programme of citizenship education, but do not need to collaborate with other schools in creating an environment in which children can interact with peers from other ethnic and/or socio-economic backgrounds. In the existing system of highly homogenous schools, legislation and an inspection framework for citizenship education about political systems and the rule of law only is unlikely to lead to more value congruence across diverse communities or changes in the socio-economic composition of the student body in a school.

Banks (2014) talks about how, in such a context, 'transformative citizenship education' offers a more viable alternative for exposing students to a more diverse set of values and cultures. Transformative citizenship education, compared to more traditional mainstream programmes such as those regulated by the Dutch Education Act, helps students acquire the knowledge, skills and values needed to function effectively within a diverse society. It also helps students to realize that 'no local loyalty can ever justify forgetting that each human being has responsibilities to every other' (Appiah, 2006, p. xvi). As citizens of the global community, transformative citizenship education helps students develop a deep understanding of the need to take action and make decisions to help solve the world's difficult problems. Transformative citizenship education allows children to participate in ways that will enhance democracy and promote equality.

Given the large autonomy of schools and parents in the Netherlands, successful initiatives to create more diverse school communities also need to come from within society, such as municipal policies to distribute children across schools 
through a centralized school-intake system, or white parents who join forces and collaboratively send their children to a predominantly black school. At present, there are few school-led initiatives, but those that have been successful would see school staff actively reach out to the local community to persuade a diverse group of parents to choose a school, or where principals have actively managed behaviour of children and their parents on the school ground in an attempt to create a culture that is more acceptable to a wider group of parents. Parents who speak multiple languages and have roots in different cultures have, in some municipalities, also acted as boundary spanners in reaching out to other ethnic or social groups and create a diverse, but cohesive school, community. These grassroots initiatives could, when incentivized and scaled up, ensure a more transformative, national, change.

\section{Notes}

1 http://wtgrantfoundation.org/library/uploads/2015/09/Inequality-is-the-ProblemPrioritizing-Research-on-Inequality.pdf

2 http://appsso.eurostat.ec.europa.eu/nui/show.do?dataset=ilc_pw03\&lang=en

3 https://www.oecd-ilibrary.org/docserver/9789264278219-en.pdf?expires= 1572094489\&id=id\&accname= guest\&checksum=A39115079D809A9A83922EF752A1B5AF

\section{References}

Altonji, J. G., \& Mansfield, R. K. (2011). The role of family, school, and community characteristics in inequality in education and labor market outcomes (pp. 339-358). In: G.J. Dunan \& R.J. Murnane (Eds.). Whither Opportunity?: Rising Inequality, Schools, and Children's Life Chances. New York: Russell Sage Foundation.

Appiah, K. A. (2006). Whose culture is it?. New York Review of Books, 53(2), 38.

Banks, J.A. (2014). Diversity, group identity, and citizenship education in a global age. Journal of Education, 194(3), 1-12.

Bax, E.H. (1988) Modernization and cleavage in Dutch society. A Study of Long Term Economic and Social Change. Groningen: Rijksuniversiteit.

Bell, C. (2005). All choices created equal? How good parents select "Failing" schools. National Center for the Study of Privatization in Education, New York: Columbia University

Boterman, W.R. (2018). School segregation in the free school choice context of Dutch cities (p. 155-179). In: X. Bonal \& C. Bellei (Eds.), Understanding School Segregation: Patterns, Causes and Consequences of Spatial Inequalities in Education. London: Bloomsbury Publishing.

Boterman W. R. (2013) Dealing with diversity: Middle class family households and the issue of black and white schools in Amsterdam. Urban Studies 50: 1128-1145.

Boterman, W. R. (2019). The role of geography in school segregation in the free parental choice context of Dutch cities. 56(15), 3074-3094. Urban Studies, 0042098019832201.

Burgess, S., Wilson, D., \& Worth, J. (2010). A national experiment in school accountability: the impact of school performance information on pupil progress and sorting: University of Bristol. Centre for Market and Public Organisation.

Collier, P. (2017). Culture, politics and economic development. Annual Review of Political Science, 20, 111-125

Dijkstra, A. B., Jungbluth, P., \& Ruiter, S. (2002) Verzuiling, sociale klasse en etniciteit. Segregatie in het Nederlandse basisonderwijs. Sociale Wetenschappen 44(4): 24-48. 
Driessen, G., \& Van Der Slik, F. (2001). Religion, denomination, and education in the Netherlands: Cognitive and noncognitive outcomes after an era of secularization. Journal for the Scientific Study of Religion, 40(4), 561-572.

Dronkers, J. (1996). Dutch public and religious schools between state and market. A balance between parental choice and national policy?. In Bildung zwischen Staat und Markt. Beiträge zum 15. Kongreß der Deutschen Gesellschaft für Erziehungswissenschaft vom 11.-13. März 1996 in Halle an der Saale (pp. 51-66).

Dronkers, J. (1999). The effects of parental conflicts and divorce on the well-being of pupils in Dutch secondary education. European Sociological Review, 15(2), 195-212.

Dronkers, J., \& Veenstra, R. (2001). Schoolprestatie-indicatoren in het voortgezet onderwijs: start, reacties en vervolg. In: A.B. Dijkstra, S. Karsten, R. Veenstra, \& A.J. Visscher (Eds.), Het oog der natie: scholen op rapport; standaarden voor de publicatie van schoolprestaties (pp. 21-36). Assen: Koninklijke Van Gorcum BV.

Duncan, G. J., \& Murnane, R. J. (Eds.). (2011). Whither opportunity?: Rising inequality, schools, and children's life chances. Russell Sage Foundation.

Forsyth, P. B., Adams, C. M., \& Hoy, W. K. (2011). Collective Trust: Why Schools Can't Improve without It. New York, NY:Teachers College Press.

Gamoran, A. (2013). Inequality Is the Problem: Prioritizing Research on Reducing Inequality. Annual Report. William T. Grant Foundation.

Gorard, Stephen. (2009) "Does the Index of Segregation Matter? The Composition of Secondary Schools in England since 1996." British Educational Research Journal 35.4 639-52.

Gilsing, R., \& Tierolf, B. (2010). Ouders nemen de wijk; in de eigen wijk naar school in gemengde wijken in Utrecht. Utrecht:Verwey-Jonker Instituut.

Goodall, C. (2015). Working with difficult to reach groups: a 'building blocks' approach to researching trust in communities (pp. 118-126). In: F. Lyon, G. Mőllering, \& M. Saunders (Eds.), Handbook of Research Methods on Trust. Cheltenham/Northampton: Edward Elgar Publishing.

Gramberg, P. (1998) School segregation: The case of Amsterdam. Urban Studies 35(3): 547-564.

Gunningham, N., \& Sinclair, D. (2009a). Organizational trust and the limits of managementbased regulation. Law \& Society Review, 43(4), 865-900.

Gutiérrez, G., Jerrim, J., \& Torres, R. (2017). School Segregation Across the World: Has Any Progress Been Made in Reducing the Separation of the Rich from the Poor?. London: UCL Institute of Education.

Inspectorate of Education (2018). De staat van het onderwijs [Annual report]. Utrecht: Inspectie van het Onderwijs.

Jerrim, J., Greany, T., \& Perera, N. (2018). Educational Disadvantage: How Does England Compare? London: Education Policy Institute.

Jongejan, D., \& Tijs, J. (2010). Prima, maar niet voor mijn kind': opleidingsniveau en houding ten aanzien van zwarte scholen onder autochtone Nederlandse ouders [level of education and attitude toward black schools among native-Dutch parents]. Migrantenstudies, 26, 2-20.

Karsten, S., Felix, C., Ledoux, G., et al. (2006) Choosing segregation or integration? The extent and effects of ethnic segregation in Dutch cities. Education and Urban Society 38: 228-247.

Karsten, S., Ledoux, G., Roeleveld, J., et al. (2003) School choice and ethnic segregation. Educational Policy 17: 452-477.

Karsten, S., Visscher, A.J., Dijkstra, A., \& Veenstra, R. (2010). Towards standards for the publication of performance indicators in the public sector: the case of schools. Public Administration, 88(1), 90-112. 
Ladd, H. F., Fiske, E.B., \& Ruijs, N. (2009) Parental Choice in the Netherlands: Growing Concerns 3092 Urban Studies 56(15) about Segregation. In: National Conference on School Choice, Vanderbilt University, Nashville, TS, October 2009. Available at: https://ncspe. tc.columbia.edu/working-papers/OP182.pdf (accessed February 2019).

Levačić, R., \& Woods, P. A. (2002). Raising school performance in the league tables (part 1): disentangling the effects of social disadvantage. British Educational Research Journal, $28(2), 207-226$.

Massey, Douglas S. and Denton, Nancy A. (1988) “The Dimensions of Residential Segregation." Social Forces 67(2), 281-315.

Mickelson, Roslyn Arlin, and Nkomo, Mokubung. (2012) "Integrated Schooling, Life Course Outcomes, and Social Cohesion in Multiethnic Democratic Societies." Review of Research in Education 36.1 197-238.

Morrone, A., Tontoranelli, N., \& Ranuzzi, G. (2009). How good is trust? Measuring trust and its role for the progress of societies. OECD Statistics Working Papers, 2009(3), 0_1

Nusche, D., Braun, H., Halasz, G., \& Santiago, P. (2014). OECD Reviews of Evaluation and Assessment in Education: Netherlands 2014. Paris, France: OECD Publishing.

OECD (2017), OECD Guidelines on Measuring Trust. Paris: OECD Publishing. http://dx.doi. org/10.1787/9789264278219-en

Oomsels, P., \& Bouckaert, G. (2017). Interorganisational trust in Flemish public administration: comparing trusted and distrusted interactions between public regulatees and public regulators. Trust in Regulatory Regimes, 80-112.

Palardy, Gregory J., Rumberger, Russell W., and Butler, Truman. (2015) “The Effect of High School Socioeconomic, Racial, and Linguistic Segregation on Academic Performance and School Behaviors." Teachers College Record 117.12 Teachers College Record, 2015, Vol.117 (12).

Parker, P. D., Marsh, H.W., Jerrim, J. P., Guo, J., \& Dicke, T. (2018). Inequity and excellence in academic performance: Evidence from 27 countries. American Educational Research Journal, 55(4), 836-858. DOI: 10.3102/0002831218760213

Reay, D., Crozier, G., \& James, D. (2011). White middle-class identities and urban schooling. Dordrecht: Springer.

Rothstein, B. (2011). The Quality of Government: Corruption, Social Trust, and Inequality in International Perspective. Chicago: University of Chicago Press.

Sampson RJ (2012) Great American City: Chicago and the Enduring Neighborhood Effect. Chicago, IL: University of Chicago Press.

Scheerens, J., Timmermans, A., \& Van der Werf, G. (2019). Socioeconomic Inequality and Student Outcomes in the Netherlands (pp. 111-133). In: L.Volante, S.V. Schnepf, J.Jerrim, \& D.A. Klinger (Eds.), Socioeconomic Inequality and Student Outcomes. Cross-National Trends, Policies, and Practices (Volume 4). Singapore: Springer.

Stolle, D. (2002). Trusting strangers-The concept of generalized trust in perspective. Österreichische Zeitschrift für Politikwissenschaft, 31(4), 397-412.

Sturm, J., Groenendijk, L., Kruithof, B., \& Rens, J. (1998). Educational Pluralism-A historical study of so-called 'pillarization' in the Netherlands, including a comparison with some developments in South African education. Comparative Education, 34(3), 281-297.

Uslaner, E. M. (2015). Measuring generalized trust: In defense of the 'standard' question, (pp. 97-106). In: F. Lyon, G. Mőllering, \& M. Saunders (Eds.), Handbook of Research Methods on Trust. Cheltenham/Northampton: Edward Elgar Publishing.

Volante, L., Schnepf, S.V., Jerrim, J., \& Klinger, D. A. (2019). Socioeconomic Inequality and Student Outcomes. Cross-National Trends, Policies, and Practices (Volume 4). Singapore: Springer.

Zeldenrijk, D. (2011). Je voelt het gewoon: Een onderzoek naar schoolkeuze en segregatie in Amsterdam-Noord. Den Haag: Raad voor Maatschappelijke Ontwikkeling. 


\title{
TRUST, PROFESSIONAL CAPACITY AND ACCOUNTABILITY IN SCHOOL IMPROVEMENT
}

\section{Austria's quality management system}

\author{
Herbert Altrichter
}

\section{Introduction}

Over the last 25-30 years, the Austrian authorities - and those of the German Bundesländer (Rürup, 2008) - have attempted to 'modernize' the governance of their education system. According to Altrichter et al. (2005) and Altrichter (2020), it is possible to identify steps of 'modernization' that are characterized by different foci of innovatory attention. Ehren and Baxter (2019, p. 2) have argued that education systems may be understood 'through the lens of trust, accountability, and capacity' (see Chapter 1 of this volume). Indeed, these waves of governance reforms are associated with changing constellations of trust between the state and its professionals in schools, the capacities attributed to them and expected of them and accountability demands

\section{Governance reforms and the relationship of trust, capacities and accountability}

In the following sections, we will describe these phases of governance changes and discuss the special constellation of trust, capacities and accountability prevalent in the respective phases.

\section{Phase 0: Stability and 'antagonistic cooperation' of state and profession}

For some time now, Austria was considered a prime example of firmly state-based and bureaucratic governance of schooling (Pavolini, 2015; West and Nikolai, 2013; Windzio et al., 2005). The central ministry was responsible for overseeing and organizing virtually all areas of school organization, classroom teaching 
and learning as well as remuneration and retirement of educational staff. Within the constitution, the central parliament set the legal framework, while detailed legislation was implemented by provincial parliaments (Eurydice, 2018). Schools were considered to be the last link in a bureaucratic chain, with little autonomy in terms of how they organize their work and curriculum.

This resulted in a legalistic, stable system, lacking in dynamism and characterized by two main governance mechanisms, i.e. a system of dual regulation (Brüsemeister, 2004): on the one hand, strong input regulation by the state, for example through central legal requirements for assessment and certification, for content specification of curricula, setting of standards for teaching material and textbooks, central funding and central teacher assignment (see Fend, 2001, p. 41). On the other hand, input regulation was coupled with a high level of professional self-regulation (or teacher autonomy) in classroom teaching with little interference by parents, school leaders (SLs) and regional administration.

In this system, parents and other stakeholders were usually kept at a distance. Parents were expected to support their children's learning, but not to participate directly in school life such as by supporting teachers in the classroom or helping out with extracurricular activities. Parent-school contacts were usually channelled through the school head or through pre-arranged consultation hours of teachers and consultation days twice a year.

Accountability was organized accordingly: Schools were monitored on their compliance to input regulations, i.e. legal requirements that specified the curriculum and extracurricular offers, while there was little monitoring of classroom teaching, and results of learning were largely attributed to children's talents and efforts.

Such a system might have been thought as being built on much trust on the professional capacities of teachers who were expected to use their relative freedom to the best of children. However, researchers such as Brüsemeister (2004) and Maroy and van Zanten (2009) did not portray this characteristic hybrid pattern of governance as a version of 'trustful collaboration' between the state and teachers, but rather conjured up an image of 'antagonistic cooperation': On the one hand, teachers had no say in strategic and resource decisions. For example, curricula, school structures and resourcing were decided by central and regional levels of school administration. On the other hand, education professionals were given much freedom in implementing these decisions in classroom teaching: '... once the teacher closes the classroom door and starts teaching, the state cannot have a say in this area' (Brüsemeister, 2004, p. 5). However, both parties, state administration and teachers, were mutually and suspiciously observing the other actor for possible infringements of one's own territory.

What are the implications of this constellation for the relationship of trust between the state and its educational professionals, the capacities attributed to them and expected of them and accountability (see Table 7.1)? The traditional model of antagonistic cooperation between teachers and state functioned within a top-down bureaucratic system: Teachers and schools were policy implementers 
TABLE 7.1 Governance changes and the relationship of trust, accountability and capacity

\begin{tabular}{|c|c|c|c|c|}
\hline & $\begin{array}{l}\text { Professional } \\
\text { Capacity }\end{array}$ & Trust & $\begin{array}{l}\text { Accountability } \\
\text { Based on ... }\end{array}$ & $\begin{array}{l}\text { Impulses for } \\
\text { Development }\end{array}$ \\
\hline $\begin{array}{l}\text { Phase } 0 \text { : } \\
\text { traditional } \\
\text { model of } \\
\text { antagonistic } \\
\text { cooperation }\end{array}$ & $\begin{array}{l}\text { Assumed - } \\
\text { capacity taken } \\
\text { for granted as } \\
\text { an element of } \\
\text { bureaucracy }\end{array}$ & $\begin{array}{l}\text { 'Antagonistic } \\
\text { cooperation': } \\
\text { top-down } \\
\text { legislation and } \\
\text { management } \\
\text { with room for } \\
\text { manoeuvre for } \\
\text { teachers }\end{array}$ & $\begin{array}{l}\text { Checks of } \\
\text { lawfulness, but } \\
\text { unquestioned } \\
\text { belief in } \\
\text { adequate } \\
\text { functioning }\end{array}$ & $\begin{array}{l}\text { Top-down } \\
\text { management is } \\
\text { seen as } \\
\text { inadequate for } \\
\text { more quickly } \\
\text { changing } \\
\text { conditions and } \\
\text { demands }\end{array}$ \\
\hline $\begin{array}{l}\text { Phase } 1+2 \text { : } \\
\text { new autonomy } \\
\text { model }\end{array}$ & $\begin{array}{l}\text { Assumed - } \\
\text { belief in } \\
\text { bottom-up } \\
\text { development }\end{array}$ & $\begin{array}{l}\text { Increased trust } \\
\text { in teachers' } \\
\text { and schools' } \\
\text { (autonomous) } \\
\text { capacities }\end{array}$ & Self-evaluation & $\begin{array}{l}\text { International } \\
\text { student } \\
\text { assessment } \\
\text { studies call } \\
\text { 'adequate } \\
\text { functioning' } \\
\text { into question }\end{array}$ \\
\hline $\begin{array}{l}\text { Phase } 3 \text { : } \\
\text { evidence-based } \\
\text { model }\end{array}$ & $\begin{array}{l}\text { Questioned } \\
\text { and checked }\end{array}$ & $\begin{array}{l}\text { 'Organized } \\
\text { doubt' in } \\
\text { teachers' and } \\
\text { schools' } \\
\text { capacities for } \\
\text { result-oriented } \\
\text { self- } \\
\text { development, } \\
\text { external } \\
\text { reform and } \\
\text { support }\end{array}$ & $\begin{array}{l}\text { External } \\
\text { evaluation }\end{array}$ & $\begin{array}{l}\text { Increasing } \\
\text { awareness of } \\
\text { the discrepancy } \\
\text { between } \\
\text { questioning } \\
\text { professional } \\
\text { capacities and } \\
\text { building } \\
\text { improvement } \\
\text { on these } \\
\text { capacities }\end{array}$ \\
\hline $\begin{array}{l}\text { Phase } 4: \\
\text { re-written } \\
\text { evidence-based } \\
\text { model }\end{array}$ & $\begin{array}{c}\text { Checked and } \\
\text { developed }\end{array}$ & $\begin{array}{l}\text { Increasing trust } \\
\text { in a system of } \\
\text { checks and } \\
\text { balances, more } \\
\text { focused } \\
\text { intervention } \\
\text { in specific } \\
\text { cases }\end{array}$ & $\begin{array}{l}\text { External } \\
\text { evaluation }+ \\
\text { self-evaluation } \\
\text { as a basis of } \\
\text { negotiated } \\
\text { development }\end{array}$ & \\
\hline
\end{tabular}

in a bureaucratic system; the focus of attention was on the input. Its output, its capacity to deliver, was taken for granted; no specific measures for comparative quality control of results and processes, i.e. of accountability, were deemed necessary. SLs had no special accountability role: They had some administrative duties in addition to their teaching but were not supposed to interfere with teachers' classroom work and to monitor the quality of teaching. There were legal and administrative checks made by regional administrators ${ }^{1}$; however, these checks were becoming fewer and fewer as the system was expanding in numbers of schools and students. Teachers (at the lower end of the hierarchy) tended to 
suspiciously watch out for infringements of their classroom autonomy (which is the 'antagonistic' element of this cooperation). Thus, 'trust at a distance' without systematically monitoring whether this trust was well founded seemed to characterize this constellation.

At the end of its era, this arrangement was challenged by teachers, politics and the public media for being too 'bureaucratic' and too slow in adapting to quickly changing local conditions and demands, for not using the energy and ingenuity of teachers for more autonomous school development (Posch and Altrichter, 1993; Schratz and Hartmann, 2009), its mediocre results in international student assessments and its administrative inefficiency, resulting from the split of responsibilities between central state and provinces (Lassnigg and Vogtenhuber, 2015). A first step of reforms to improve these issues will be described in the next section.

\section{Phases 1 and 2: School autonomy reforms and in-school coordination}

In the early 1990s, school autonomy policies aimed to expand the individual school's scope for action and its right to make decisions, to make it more responsive to the potential capacities and needs of its catchment areas, which in turn was meant to increase the quality and efficiency of work at schools. In Austria, the most important instrument for 'school autonomy' was the curriculum: Schools could rewrite $5-10 \%$ of their curriculum according to their preferences. Via this mechanism schools aimed to attract students in an increasingly competitive environment - caused primarily by decreasing student numbers. Additional autonomy for deciding the length of the school day, internal organization, finances and internal staffing (but not personnel recruitment) were gradually given to schools (see Altrichter et al., 2016).

In a second wave of reform between 1995 and 2000, in-school and between-school coordination were addressed: First, the capacity and rights in regard to the management of individual schools were strengthened (school management training, strengthening SLs' rights), and instruments for internal management (such as school development plans [DPs], in-school quality management) were introduced (Altrichter et al., 2005). Second, central and regional administrative authorities were looking for new ways to 'orchestrate the variety' that school autonomy policies had apparently produced (EDK, 2000). New forms of system monitoring and school supervision were developed; set assessment tasks and benchmarking looked to make assessment criteria comparable in different schools.

What are the implications of these reforms for the relationship of trust, professional capacity and accountability? The new autonomy model that evolved through reform phases 1 and 2 (see Table 7.1) was based on trust in teacher professionalism and, particularly in wave 2 , on trust in the newly evolving role of school leadership. Schools could only make use of autonomous opportunities, e.g. for curriculum development, if their staff possessed the necessary professional capacities, 
if school management assumed an active leadership role and if it motivated staff for participating in school improvement processes. Accountability entered the field via the medium of self-evaluation. The assumption underlying (e.g. Rauscher, 1999) was that an autonomously developing school would be interested in self-evaluating to find out if its improvement goals had been reached and its energies were focused effectively.

This constellation was challenged when it became apparent that schools differed in their developmental capacities, i.e. that not all teachers and SLs could live up to the wealth of competencies expected of them in the previous paragraph. Furthermore, many schools used the new 'curricular autonomy' for developing school-specific curricula and school profiles, which were meant to attract a larger number of more able students. In this way, they focused part of their efforts on competing with other schools and not necessarily on producing better learning conditions for students in areas of national interest (Altrichter et al., 2014). Ultimately, Programme for International Student Assessment (PISA) results raised public doubts over whether the trust in the system's quality, in self-developing schools and in the capacity of their staff was always, well founded (see next section).

\section{Phase 3: The 'PISA shock' and evidence-based governance models}

In 2001, the so-called PISA shock hit education debates in Germany and (initially, to a lesser degree) Austria: Their school systems had performed less well than expected in the international comparative student assessment. It sparked an acute and sometimes feverish political and media debate that paved the way for more thorough changes, for a third wave of governance reforms. The authorities responded by gradually developing and implementing a type of evidence-based governance model characterized by the following features (Altrichter and Gamsjäger, 2017): (1) setting (output) goals more clearly than before, (2) measuring goal achievement, (3) feeding back achievement results to various actors on all system levels to stimulate improvement and (4) involving stakeholders more than before in order to increase pressure on and/or support for schools.

The most important practical incarnations of evidence-based reforms were to be found in the performance standard and school inspection policies. A performance standard policy was introduced into the Austrian education system in 2008 (Altrichter and Gamsjäger, 2017) with performance standards for the primary cycle of schooling (students of 10 years in Math and German) and the lower secondary cycle (students of 14 years in Math, German and English). Classroom material, diagnostic tests and sample test items were offered alongside other publications and workshops in order to support full implementation of the new standards and of competence-based teaching in classrooms. Nationwide comparative standard testing was administered for the first time in May 2012, and performance results fed back to students, parents, teachers, SLs and administrators. 
Furthermore, school inspections were introduced as a second element of evidence-based reforms. ${ }^{2}$ While performance standards and feedback emphasize comparative data of student performance tests and output criteria, most inspection models centre on teams of inspectors collecting and evaluating different kinds of evidence; they may also include process criteria and take account of specific local conditions for schooling and improvement (Rürup, 2008). Contrary to the school systems of many German Bundesländer and Swiss cantons, Austria did not introduce a statewide system of 'inspections'. There were experiments with new inspection models in some provinces (Altrichter, 2017; Ehren et al., 2013), which were loosely based on German models. Eventually, the Austrian authorities decided to go for an alternative model of systemic quality management that will be described in more detail in the 'SQA: The new systemic quality management policy' section.

The evidence-based model marked a sharp contrast to the previous phases in terms of the relationship of trust, professional capacities and accountability (see Table 7.1). Trust in teacher and SL professionalism was undermined by disappointing comparative performance results and by slow 'autonomous' school improvement (which, additionally, was said to focus too rarely on the 'really essential' issues of student learning and performance). As a consequence, the locus of control shifted away from schools and educational professionals, who were thought to lack motivation and/or diagnostic and innovative capacity for fast and well-focused innovation.

The heated debate in response to PISA pressured the authorities to take immediate action to improve performance (Tillmann et al., 2008) and to protect public trust in the school system. Mechanisms monitoring system performance, such as inspections and performance assessment, were introduced because they were considered to allow for more informed and evidence-based decisions in the improvement of schools and teaching quality and to be more effective means to effect. However, these control mechanisms had also some potential for substituting the potentially decreasing public trust.

Control mechanisms may substitute trust between actors in cases where there is no or not enough trust existing; in this perspective, trust and control are alternative routes for arriving at stable social relationships (Gulati, 1995). Control may also be complementary to trust in that trust and control can build on and reinforce assumptions of perceived trustworthiness (Costa and BijlsmaFrankema, 2007).

However, control mechanisms may also damage trust within relationships. For Das and Teng (2001), this is connected with the type of control: Formal control through strict rules and objectives means may undermine trust because it harms actors' autonomous decisions about what works best. Social control through shared goals and norms may complement trust by increasing mutual understandings. Other researchers argue that it is not merely the type of control mechanisms that makes control harmful or not but also the dynamic process by which the relationship of trust and control unfolds, its contextual setting and 
the way control is enacted by supervising actors (Costa and Bijlsma-Frankema, 2007). In sum, substitutability and complementarity of trust-control relations seem to be connected with the overall appraisal processes by which actors evaluate their agency in a given place and time rather than with the objective characteristics of the governance constellations (Young, 2003, p. 90).

While evidence-based reforms seem to be implemented to counter potentially decreasing public trust in schools and in the teaching profession, they themselves are firmly built on 'trust in the rational state' that, in developing nationwide assessment instruments and in producing comparative performance data, is designed to help to base improvement decisions on evidence-based approaches. Accountability was targeted at acquisition of the best possible system-wide (and, if possible, also locally focused through school inspections) information (untainted by teacher reservations and opinion) in order to detect deficiencies at all levels and tailor both resolute system-wide reforms and site-focused interventions - an area in which teachers, in many cases, lacked capacity.

Thus, an idea of 'rationalizing governance' or of 'evidence-based selfgovernance' seems to be at the heart of the evidence-based model: Improvement decisions on individual school level, but also on all other levels of schooling, were no longer to flow from the political preferences or diffuse value judgements, but were to be based on empirical, hard facts: The evidence-based philosophy promises to steer through insight (Rürup, 2008); facts are so compelling that 'actors cannot escape this evidence' (Heinrich, 2018, p. 327).

This set up was challenged when it became apparent that the evidence-based instruments of inspections and performance assessments did not bring resolute and well-focused improvement as quickly as expected and that they could not function without professional participation of teachers and SLs, which will be explained in the next section in more detail.

\section{Phase 4: Criticism and re-written versions of evidence-based governance models}

In recent years, criticism of the evidence-based strategy has been increasing in German-speaking countries (e.g. Bellmann, 2016). A line of epistemological critique pointed to the flawed promise that externally developed, general answers were best for specific contexts and complex interactions (Berliner, 2002; Biesta, 2007; Heinrich, 2018). Another line of criticism argued that comparatively high investments (Fend, 2018) were required for establishing the instruments of evidence-based governance - investments that went into the infrastructure of education with no direct benefit for the educational processes in classrooms (Piezunka, 2019, p. 271). Additionally, these instruments did not fully deliver what was expected (Heinrich, 2018, p. 327; Piezunka, 2019, p. 271). Data feedback from national comparative performance tests rarely stimulated classroom improvement beyond superficial changes or reteaching (Altrichter et al., 2016; Maier and Kuper, 2012). School inspections seemed to be more effective in communicating 
normative expectations to schools rather than in stimulating school improvement (Gustafsson et al., 2015). These problems may be connected with simplistic models of evidence transfer and use (Maritzen, 2018), and more generally with the ambivalent attitude towards professionalism inherent in the evidence-based strategy.

The evidence-based philosophy seems to be characterized by a fundamental tension between organized doubt of professional competence and transfer of complex additional tasks to the profession. On the one hand, the evidence-based strategy was partly born out of the doubt that the teacher-centred and schoolbased development strategy of phases 1 and 2 would address the right goals and would rapidly pursue them. Centrally prescribed objectives and specific directions for improvement were to replace lengthy and unsecure negotiation processes at school sites, and control mechanisms were meant to substitute mere trust in the profession. At the same time, the evidence-based strategy cannot be put into practice other than by professional actors: by teachers who show enough competence and initiative to draw appropriate consequences from educational standards and data feedback; by SLs who are ready to take on responsibility for their students' results and to put energy into further development (see Altrichter and Geisler, 2012).

These conflicting approaches to teacher capacity were reflected by complaints by teachers and teacher associations about the large workload for schools associated with inspections and performance assessment (Piezunka, 2019, p. 271). What was meant to bolster public trust in the school system, was in danger of arousing 'teachers' feelings of being mistrusted by both the school provider and parents' (Hult and Edström, 2016, p. 321) and, as a consequence, of damaging the trust between the profession and the state.

This situation may also be interpreted as struggle between two concepts of professionalism (Gewirtz et al., 2009): While Austrian teachers may have largely worked according to an image of traditional professionalism characterized 'by low or ritualized collaboration and a preference for the role of the lone fighter' (Nairz-Wirth and Feldmann, 2019, pp. 4 and 7), the evidence-based reform assumes to strengthen alternative types of 'new professionalism' (Day and Sachs, 2004) based on 'openness to science and research findings, high levels of interest in collaboration and innovation' (Nairz-Wirth and Feldmann, 2019, p. 7).

Even if criticism of the evidence-based strategy has significantly increased in recent years and even if there is some weakening of its groundwork, there is no new comprehensive paradigm arising in the educational discourses of the German-speaking countries which can replace evidence-based control. The idea of evidence-based governance seems to continue to govern education policy in general, while there have been many changes to specific aspects and arrangements of the evidence-based governance instruments. These changes may be seen as a fourth wave of governance reforms in which the early versions of evidence-based instruments are re-written.

A good example are inspection reforms that have taken place recently in many countries. In their logic and aspirations (not necessarily in their results), these 
changes to differentiated, more focused inspection approaches ('proportionate' or 'riskbased inspections'; see Ehren and Honingh, 2011; Honingh et al., 2018, for the Netherlands; Baxter, 2014, for England) may be read as strategies to cope with these challenges of cost and professionalism. In these approaches, resources are bundled and focused on schools with problems, while strong schools are considered to be 'professional' enough to be allowed more room for manoeuvre and to self-determine their development efforts. In England, however, this has since backfired as some schools deemed outstanding, have not been inspected since, yet parents report that standards have slipped since the original grade. ${ }^{3}$

Similar developments may be observed in German-speaking school systems. 'New inspections' were rapidly introduced as a core element of the new governance models in virtually all German Bundesländer (Kotthoff et al., 2016; Piezunka, 2019). In recent years, some inspectorates have again been abolished (e.g. between 2010 and 2017 by Schleswig-Holstein, Rhineland-Palatinate and Hesse), but even in pioneering school systems such as Lower Saxony, there have been changes in inspection procedures (in 2013 and again in 2018; see Sowada and Dedering, 2016), which seem to revolve around two key aspects: controlling costs and involving teachers and SLs as active professionals in the procedure rather than having them as dependent recipients of external advice and, at the same time, putting additional workload on their shoulders - i.e. around features that have been identified as most critical issues of the original evidence-based strategy above. These measures were also meant to ease the workload for teachers and SLs, which another set of changes in 2018 again aimed for (Tonne, 2018).

Evidently, there is a 'dialogic turn' (Heinrich, 2018, p. 325) in German inspections. What is ultimately considered relevant 'evidence' for school improvement is not produced by the elaborate inspections procedures, but relocated to the school level and constructed in the participative 'dialogues' of teachers and inspectors. In a recent study, Piezunka (2019) interviewed 18 staff members of German school inspectorates. She found that these inspectors struggled to make their inspections more acceptable for schools by using two main strategies: They 'changed the presentation of their data (more descriptive, less evaluative) and gave school representatives a greater say when formulating expectations' (Piezunka, 2019, p. 282).

Although Piezunka's (2019, p. 283) argument is on an interactional level, these inspectors' strategies of coping with teacher criticism seem to mirror the thrust of institutional policy reforms. The author points to a potential paradoxical effect that may also be relevant at institutional level: In the face of criticism over the efficiency of its systems, the inspectorate aims to enhance its impact for school improvement by softening its approach and giving schools more autonomy and say. In so doing, they may compromise their regulatory distance and risk being accused of inspector capture.

What are the implications for the relationship of trust, professional capacities and accountability (see Table 7.1)? The re-written evidence-based model is still 
using evidence-based instruments (e.g. by performance standard testing); however, it applies them more differentially for its monitoring and intervention strategies. Since a relevant section of the teaching force seemed to oppose the evidence-based instruments (by not using them, by complaining about additional workload and decreasing trust), the re-written strategy is to win them by signalling that accountability is not meant to substitute, but to complement trust in teachers' professional capacity. However, thanks to the increased public knowledge about school performance through the PISA debate, communicating boundless trust in schools and professionals would not be credible. Thus, the new strategy is built on a concept of 'differential trust' in the system actors. Both risk-based inspection approaches and the new Austrian quality management strategy (see next section) assume that there are teachers and schools who have the will and the professional capacity for self-development when subjected to a minimal version of monitoring. For these organizations and individuals, quality improvement can primarily build on professional agency which is balanced by some differential monitoring that serves both as a detection mechanism for unwanted development trajectories and a motivational stimulus for continuing improvement.

The assumption, however, is that there are also other schools and teachers who need more monitoring, support and guidance. They are more often subjected to inspections by proportionate inspection models, and their development activities are more closely monitored by the authorities. Those who are capable of and motivated to change must be encouraged and enabled to self-manage, evaluate their provision and outcomes and develop their school, while those who are not must be subjected to more focused intervention in order to restore and uphold their functioning.

\section{SQA: The new systemic quality management policy}

In this section, we will examine the Austrian systemic quality management policy SQA ('Schulqualität Allgemeinbildung'; which translates as 'school quality in general education') that may be understood as another example for a 're-written evidence-based governance model'.

In the wake of the PISA shock (see 'Phase 3: The 'PISA shock' and evidence-based governance models' section), the school administration bodies in some Austrian provinces began to discuss alternative ways of fulfilling their functions and to search for a new balance between administration, quality control and developmental support (Altrichter, 2017). Since the school year 2005/2006, some provinces experimented with 'new inspection models'. However, these were never institutionalized by federal legislation. Amidst diverging developments in the different provinces, the legal framework for quality assurance in the Austrian education system, the Federal School Supervision Act (BSAG [Bundes-Schulaufsichtsgesetz], 1962), was completely revised (BSAG, 2011) in spring 2011. A National Quality Framework (Nationaler Qualitätsrahmen) was spelt 
out, which was to organize quality monitoring and quality development in the Austrian school system by the following elements:

- A 'definition and description of school quality' clarifies the goals of development and the criteria of evaluation.

- A 'system of planning and reporting' by which all levels of the school system (from central administration to individual schools) periodically (annually to every third year depending on the school type) plan and evaluate their development.

- 'Periodical target agreements' between all levels of the school system which clarify (national, regional and school specific) goals, measures and expected performance.

- The obligation of the national authorities to provide instruments for governance and (self) evaluation and support structures for schools.

In order to implement the new quality management strategy, the ministry launched the nationwide programme $S Q A$ that has been put into practice since 2013/2014. A central element of SQA is the DP (Entwicklungsplan). These development plans are - in the official rationale - both 'working instruments for goal- and result-oriented planning and implementation of educational measures' and central instruments of accountability (Radnitzky, 2015, p. 9). The individual school's DP must specify two 'themes' (at minimum) that it aims to promote during the next development period. The first theme is nationwide, obligatory and phrased by the ministry; for the school year 2015/2016, it was 'further developing learning and teaching with respect to individualization and competence-based teaching in inclusive settings' (see BMBF, 2015b). The second theme (and all further voluntary themes) is chosen by the individual school according to need and interest.

SQA mandates a minimum content of DPs: For every theme, the plan must include some evaluation of the present state, goals, indicators and measures for reaching the goals (including a plan for evaluation of development measures), a professional DP, and information with respect to processes, responsibilities and structures of the overall development effort (see BMBF, 2015c). The planning period is 3 years; an annual update is required which must be sent to the regional administrator. DPs will also be written on district, province and central state level and summarized in a national education report (see BMBF, 2013, p. 2).

The second central element are conversations on results and target agreements (Bilanz- und Zielvereinbarungsgespräche; BZGs). These target agreements take place between leading individuals on all levels, e.g. between a SL and the regional administrator, between the province chief administrator and the respective department head in the ministry. They aspire to 'dialogue-oriented leadership' and are to be conducted as dialogues of equal partners. In these target agreements, the DP will be discussed, and if necessary, amended; thereby, directions for future school development are agreed upon (see BMBF, 2015a). 
Finally, SQA also offers a support structure for quality evaluation and development of schools (see Radnitzky, 2015, p. 11). There is an electronic webpage (www.sqa.at) that gives access to various information documents, development and evaluation instruments and a pool of consultants for school and classroom development. The implementation concept includes workshops for inspectors and school principals. A network of coordinators on school, province and system level is to support further implementation.

A very special support feature is a new role created at the school level: A teacher is appointed by the SL to serve as an SQA coordinator (SC). ${ }^{5}$ While the overall 'strategic responsibility for quality development' as well as the responsibility for preparing DPs and for target agreement discussions (BZG) lies with the SL, the SC's task is to support 'school leaders in the operational implementation of SQA at the school site' by taking over 'partial responsibility' for the quality development processes (BMBF, 2016a).

What is the main rationale of this 'quality model'? Provincial administrations had experimented with 'new inspection models'. The new legislation deprives these models of their legal basis and wants to provide an alternative route to quality management because inspection, in the interpretation of ministry officials, is too intrusive and disenfranchising. The 'National Quality Framework' aims for improvement cycles starting from self-evaluations of schools (rather than from external evaluations) and from self-devised DPs (rather than from DPs that responded to external feedback). It aims for 'strengthening commitment' of the profession and 'balancing of interests between nation-wide frame requirements, regional needs, and personal aspirations' (see Radnitzky, 2015, p. 9). In many places, the transactions between different system levels (e.g. between SLs and regional administrators) are portrayed as 'dialogues of equal partners'; the whole procedure is to signal respect for and trust in the professional capacities of teachers and SLs.

Through SQA, the authorities are promoting a complementary perspective of trust and control that may be seen as rowing back from the phase 3 position of strong external accountability and trying to win teachers who had been in a strong position of relative independence and did not participate in phase 3 innovations as expected. Performance standard testing and feedback procedures are still in place, and schools are expected to refer to them in their DPs. The body of regional administrators continues to exist (being renamed 'regional school quality managers'). Their function under the new legislation (alongside their continuing administrative duties) lies in negotiating target agreements with individual schools. Thus, they continue in their intermediary role of linking education system levels but use 'more modern and dialogic' instruments of target negotiation and agreement.

In his analysis of the new approach, Maritzen (2015, p. 15) interprets SQA as an institutional way of coping with the effects and repercussions of the previous Phase 3 reform when 'a technologically defined programme of education monitoring had been transplanted into a system largely unprepared for that'. Indeed, nationwide performance testing and data feedback were alien innovations for most teachers who were not educated for self-confident use of these instruments 
and suspected to come under closer surveillance (Gamsjäger et al., 2019). The SQA reform is meant to work against impending teacher alienation. It aspires to provide both 'mutual communication between system levels' and 'reversibility of relationships', but also fixed formats that are to lead to 'binding agreements and reliable commitment'. The scheme addresses teachers and SLs as professionals, but also implies that further professionalization (for instance with respect to mid-term school development planning, in-school coordination and evaluation) is necessary (Maritzen, 2015, p. 16).

\section{Challenges and pitfalls of a 're-written evidence-based policy'}

The argument so far has been that school systems such as the Austrian have recently seen changes of their governance that were on the one hand motivated by changing levels of trust in teachers and an understanding of the capacity they needed to engage with external accountability and use evidence to improve, while similarly affecting how schools and teachers are held to account, the capacity (and particularly information and use of evidence-informed standards) they have to use to improve and who and what sources of information are to be trusted to improve teaching and student outcomes. The recent 're-written evidence-based model' of systemic quality management policy is characterized by the following features (see 'Phase 4: Criticism and re-written versions of evidence-based governance models' section): (1) by distinguishing between schools with more or less professional capacities, (2) by investing more trust in the 'more professional schools' and giving them more leeway in using relevant data and deciding on their future development and (3) by applying closer control and guidance on 'less professional schools', these models aim (4) to improve the effectiveness of evidence-based instruments for enhancing school quality and (5) to control costs and make these models more cost-efficient. By a concept of 'differential trust' $(=1)$, this strategy tries to counter criticism of damaging trust in teachers and schools and motivate education professionals for engaging themselves in a complementary model of trust and accountability $(=2)$. However, this strategy is not plausible for all schools, since the PISA debate has unearthed serious doubts in the performance of, at least parts, the school system. In order to reach the overall goals (=4 and 5 ), underperforming schools have to be singled out and subjected to a different treatment, i.e. to a more focused intervention (=3) that is built on a substitutional concept of trust and accountability.

The Austrian quality management system SQA is analysed as an example for these models ('SQA: The new systemic quality management policy' section). However, what has been explained before are aspirations of a policy model that need not necessarily be put into practice. Thus, in the concluding section, we will summarize early quantitative (Kemethofer and Altrichter, 2015; Skliris et al., 2018) and qualitative (Altrichter et al., 2020) evidence of the SQA implementation to discuss whether or not a phase 4 model such as SQA is perceived 
by teachers and SLs as trustful in their capacities and whether or not it opens up professionals for accountability more than before.

The trust-building message of the reform is only taken up by parts of the profession, and in particular by SLs; however, many teachers remain sceptical and do not see 'trust in their professional capacities'. In quantitative studies, the majority of SLs and SCs think that SQA is a useful instrument for quality assurance and development in schools, while about 10\% deny its useful potential (Kemethofer and Altrichter, 2015; Skliris et al., 2018). However, the cost-benefit relationship is seen more critical: About $10 \%$ of SCs and SLs say that there is a positive cost-benefit relationship, while more than a third say that costs are higher than benefits and many respondents remain neutral (Skliris et al., 2018, p. 15). This relationship is evaluated more positively by higher administrative ranks: About a third of the regional administrators and of the SCs on province level say that there is a positive cost-benefit relationship, while about $10 \%$ give a negative evaluation of this relationship (Skliris et al., 2018).

None of the SLs in a qualitative study of five secondary schools (Altrichter et al., 2020) rejects SQA; all consider it a reasonable and useful strategy for pushing forward with school improvement, although some think it is demanding in terms of times and resources. The new role of SCs is generally well received: They support the SLs and build a communicative bridge to staff which may increase SQA acceptance by staff. Particularly, the target agreement conversations are described as 'dialogic', 'non-hierarchical' and 'confidence-based'. The message that school improvement needs time and precedes inspection and evaluation seems to sink well with SLs. All these observations seem to indicate that a relevant section of SLs feel that the quality development system invests some trust in their capacities.

There are no quantitative data about teachers' reactions; however, the qualitative study (Altrichter et al., 2020) indicates that many teachers are still sceptical of the 'structuredness' of the quality system, and in particular, of its accountability and evaluation elements. Their reservations are directed to the inherent distrust, and constraint of individuality; they see it as 'state coercion' and threat to their professional self-determination. They see excessive work demands and emphasize that they are not being trained for these demands. Teachers do not experience themselves the target agreement conversations between SLs and regional administrators which are meant as an exemplar of the more trustful relationships between school and administrative level. Even the new management system's central offer to start improvement processes with their own goals and with self-evaluation does not change a great deal for teachers, because many teachers may engage in developing their teaching, however, shy away from seeing the need for coordinated wholeschool improvement. And they certainly do not feel equipped for evaluation at all which prevents them from taking ownership of evaluative efforts in their school.

What is the relationship of the SQA version of accountability to capacity and professionalism of teachers and schools? It seems that accountability measures are characterized by some in-built tension between critical scrutiny of and trust in the professional capacities of teachers. On the one hand, school outcomes are 
checked and the processes and teacher competencies invested are questioned in order to detect areas in need of improvement; on the other hand, teachers and schools are trusted that they will develop their professional competencies and find ways for improvement. As a consequence, accountability systems may differ in the extent to which users experience this tension, either as a painful ambivalence or as a liveable balance of diverse work aspects. Whether or not accountability and trust are seen in a substitutional or complementary relationship depends much on the dynamics of implementing these governance instruments in a given context. Through SQA the authorities aimed to promote a complementary concept of accountability and trust in the profession.

For the SLs in this study, SQA seems to support their professionalism. The quality management system obliges them to push forward with well-focused and structured school improvement which would be expected anyway from the leadership role and which (in the case schools) they aim for anyway. The SQA process offers them a useful way of structuring the improvement process, the regional SQA meetings provide some information and exchange with fellow principals and the hierarchical obligation to deliver helps them to convince staff to join in.

However, it is not difficult to imagine cases (which were not in the case sample of Altrichter et al., 2020) in which the SQA process cruelly exposes the lack of leadership capacities. If so, then it would also become obvious that few opportunities for developing or substituting lacking leadership competencies are available within the SQA structure; individuals aiming for additional professional skills would have to actively search for additional courses offered by the regional professional development institutions. Thus, SQA seems to build on basic SL capacities and support them but includes only minor provisions for developing them. As a consequence, it is questionable if the SQA model includes an effective operationalization of 'differential trust in diverse professional competencies' that has been claimed as a characteristic of phase 4 strategies. Certainly, regional administrators may adapt their interventions to the specific situation of the individual school in the target agreement conversations; however, the case study data does not suggest that this is the case.

It is difficult to judge by case study data alone; however, there is much indication that the SLs' and schools' prior experience with school development and their knowledge and skills are a precondition and predictor for successful implementation of a sound version of this quality management strategy. Thus, the hypothesis is that SQA builds on professional capacities and strengthens them where they are, but it will have difficulties in building up capacity where knowledge and skills are lacking from the outset.

Through the quality management system authorities try to communicate a complementary idea of trust and control: teachers are addressed as competent professionals who must critically examine their contribution to student learning and improve their competence and practice accordingly. Not all teachers (interviewed in case schools) are responding to both sides of the message, but rather continue to see accountability as distrust and constraint (e.g. Hult and Edström, 
2016, report similar results for Sweden). However, some teacher statements seem to point to development: They say that the commitment to the school's quality goals, the extent of educational communication among staff and of reflection of one's teaching have increased in the course of the SQA process. Some SLs interpret these observations as indications for developing teaching competency through reflection and for increasing professionalism.

Another realm of professional capacity is left open: evaluative capacities. Aspects of evaluation and accountability had not been emphasized in the communication of the reform, but many teachers remain still very attentive to its possible function of evaluating their work. Downplaying the evaluative aspect in the implementation of SQA may have contributed to some vagueness or uncertainty about what evaluation means and what kind of evaluation is meant by SQA. As a consequence, both teachers and SLs seem uncertain in what way to cope with SQA evaluation tasks. There is certainly much online material available; however, many professionals seem to lack basic concepts and competencies that would enable them to make deliberate use of online support.

\section{Conclusion}

To sum up, the rationale of the new Austrian quality management system SQA (which is taken as an example for a 're-written evidence-based model') is repositioning teachers, principals and local/national authorities as actors in the governance of schools. The system is set up in a way where the latter two are now becoming more powerful and teachers less so. It requires principals and authorities to agree on change in 'dialogic' target conversations. Binding these two role holders together creates a counterbalance for the strong autonomy of teachers and their lack of engagement in evaluation and accountability.

The early data available for its implementation indicates that many teachers seem to hesitate to buy into the language and instruments of coordination, management and evaluation that are communicated by SQA. Teachers do not feel well prepared for evaluation, and they are far off the central trust-building processes of SQA that are usually taking place between SLs and regional administrators. And they seem to be suspicious of the trustful conversion of accountability instruments that were originally introduced in the third wave of reform of German speaking school governances to assess school quality from an external, more distanced perspective rather than from the hitherto purely internal perspectives of quality appreciation (Piezunka, 2019).

The SLs seem to welcome the change because they are offered useful things by SQA: strengthening and legitimation of their role and improvement efforts towards teachers, practical procedures and instruments for orchestrating improvement processes that they are responsible for anyway. Additionally, they are given support by the new role of SCs. They can test the 'dialogic' claims of SQA in their encounters with the regional administrators and, in the majority of the documented cases, judge these 'dialogues' to be trustworthy. 


\section{Notes}

1 These persons are called in Austria 'Schulinspektor' and have both administrative (e.g. personnel recruitment and distribution) and evaluative functions. We use the term 'regional administrator' to avoid their role being equated to 'new inspectors' in the international usage of the term.

2 School inspections have been in operation for about 200 years in some countries (e.g. in Austria; see Ehren et al., 2013). Their way of working has evolved over time, but traditionally they were combining evaluative and administrative functions in different constellations. The term 'new inspections' refers to recent inspection models which are usually characterized by concentrating on evaluative functions (as opposed to administrative), explicit quality criteria and procedures, and by using instruments borrowed for social research (such as interviews, observation rubrics; see Altrichter and Kemethofer, 2016).

3 See https://www.cypnow.co.uk/cyp/news/2006280/inspections-of-outstanding-schoolsreveal-falling-standards.

4 For vocational schools, the existing programme 'Quality in vocational education' (Qualität in der Berufsbildung), which was compatible with the provisions of the new law, was used for implementation (see BMBF, 2014).

5 In all schools with more than four classes; smaller primary schools may form a network that appoints an SC (BMBF, 2016b).

\section{References}

Altrichter, H. (2017). The Short Flourishing of an Inspection System (pp. 206-230). In: J. A. Baxter (Ed.), School Inspectors: Policy Implementers, Policy Shapers in National Policy Contexts. Cham: Springer.

Altrichter, H. (2020). Changing Contexts of School Governance: The Emergence of Evidence-Based Governance Models in the State-Based Education Systems of Austria and Germany. In:J.Allan,V. Harwood, \& C. Rübner Jørgensen (Eds.), Schooling Governance. World Yearbook in Education 2020. London: Routledge (accepted for publication).

Altrichter, H., Brauckmann, S., Lassnigg, L., Mossbrugger, R., \& Gartmann, G. B. (2016). Schulautonomie oder die Verteilung von Entscheidungsrechten und Verantwortung im Schulsystem (pp. 263-303). In: M. Bruneforth, F. Eder, K. Krainer, C. Schreiner, A. Seel, \& C. Spiel (Eds.), Nationaler Bildungsbericht Österreich 2015 (Volume 2). Graz: Leykam.

Altrichter, H., Brüsemeister, T., \& Heinrich, M. (2005). Merkmale und Fragen einer Governance-Reform am Beispiel des österreichischen Schulwesens. Österreichische Zeitschrift Für Soziologie, 30(4), 6-28.

Altrichter, H., Ettl, K., Grinner, K., Kolleritsch, K., Kopp-Sixt, S., Leeb-Brandstetter, R., Pöschko, H., \& Postlbauer, A. (2020). Implementing a New Quality Management Strategy in a School System: Repercussions for the Relationship of Trust and Professional Capacities. (Under review).

Altrichter, H., \& Gamsjäger, M. (2017). A conceptual model for research in performance standard policies. Nordic Journal of Studies in Education Policy, 3(1), 6-20.

Altrichter, H., \& Geisler, B. (2012). Unterrichtsentwicklung in Zeiten der Systemreform. Schweizerische Zeitschrift Für Bildungswissenschaften, 34(1), 71-95.

Altrichter, H., Heinrich, M., \& Soukup-Altrichter, K. (2014). School decentralisation as a process of differentiation, hierarchization and selection. Journal of Education Policy, 29(5), 675-699.

Altrichter, H., \& Kemethofer, D. (2015). Does accountability pressure through school inspections promote school improvement?. School Effectiveness and School Improvement, 26(1), $32-56$. 
Altrichter, H., \& Kemethofer, D. (2016). Stichwort: Schulinspektion. Zeitschrift Für Erziehungswissenschaft, 19(3), 487-508.

Altrichter, H., Moosbrugger, R., \& Zuber, J. (2016). Schul- und Unterrichtsentwicklung durch Datenrückmeldung (pp. 235-277). In: H. Altrichter, \& K. Maag Merki (Eds.), Handbuch Neue Steuerung im Schulsystem. Wiesbaden: SpringerVS.

Baxter, J. A. (2014). An independent inspectorate?. School Leadership \& Management, 34(1), 21-38.

Bellmann, J. (2016). Datengetrieben und/oder evidenzbasiert?. Zeitschrift für Erziehungswissenschaft, 19(1), 147-161.

Berliner, D. C. (2002). Educational research:The hardest science of all. Educational Researcher, $31(8), 18-20$.

Biesta, G. (2007). Why 'what works' won't work: Evidence-based practice and the democratic deficit in educational research. Educational Theory, 57(1), 1-22.

BMBF. (2013). Entwicklungsplan: Merkmale. Retrieved from http://www.sqa.at/pluginfile. php/951/mod_folder/content/0/merkmale.pdf.

BMBF. (2014). QIBB - Wie \& Was?. Retrieved from https://www.qibb.at/de/ueber_qibb/ qibb_wie_was.html.

BMBF. (2015a). Allgemeines zu Bilanz- und Zielvereinbarungsgesprächen. Retrieved from http://www.sqa.at/course/view.php?id=62.

BMBF. (2015b). SQA auf einen Blick. Retrieved from http://www.sqa.at/course/view. php?id=151.

BMBF. (2015c). Struktur von Entwicklungsplänen. Retrieved from http://www.sqa.at/ course/view.php?id=21.

BMBF. (2016a). Aufgabenprofil der SQA-Schulkoordinator/innen. Retrieved from http://www.sqa.at/pluginfile.php/2059/course/section/1121/Aufgabenprofil_ SK_19032016\%20fin\%20(1).pdf.

BMBF. (2016b). Rundschreiben Nr. 6/2016: Schulentwicklung mit SQA. Retrieved from http://www.sqa.at/pluginfile.php/2068/course/section/1132/SQA\%20 Rundschreiben\%206-2016.pdf.

Brüsemeister, T. (2004). Das andere Lehrerleben. Bern: Haupt.

BSAG [Bundes-Schulaufsichtsgesetz]. (1962). BGB1. Nr. 240/1962 i.d.F. BGBl. I N5. 25/2008. Retrieved from http://www.ris.bka.gv.at/GeltendeFassung.wxe?Abfrage= Bundesnormen\&Gesetzesnummer=10009264\&ShowPrintPreview=True.

BSAG (2011). Änderung des Bundes-Schulaufsichtsgesetzes. BGBl. I Nr. 28/2011 vom 20. Mai 2011. Retrieved from https://www.ris.bka.gv.at/Dokumente/BgblAuth/ BGBLA_2011_I_28/BGBLA_2011_I_28.pdfsig.

Costa,A. C., \& Bijlsma-Frankema, K. (2007). Trust and control interrelations: New perspectives on the trust-control nexus. Group \& Organization Management, 32(4), 392-406.

Das, T. K., \& Teng, B. S. (2001). Trust, control and risk in strategic alliances: An integrated framework. Organization Studies, 22, 251-283.

Day, C., \& Sachs, J. (2004). Professionalism, Performativity and Empowerment (pp. 3-32). In: C. Day, \& J. Sachs (Eds.), International Handbook on the Continuing Professional Development of Teachers. Maidenhead: Open University Press.

EDK. (2000). Die Vielfalt orchestrieren. Innsbruck: StudienVerlag.

Ehren, M. C. M., Altrichter, H., McNamara, G., \& O’Hara, J. (2013). Impact of school inspections on improvement of schools. Educational Assessment, Evaluation and Accountability, 25(1), 3-43.

Ehren, M. C. M., \& Baxter, J.A. (2020). Trust, Accountability and Capacity:Three Building Blocks of Education System Reform. Cpt. in this book. 
Ehren, M. C. M., \& Honingh, M.E. (2011). Risk-based school inspections in the Netherlands. Studies in Educational Evaluation, 37, 239-248.

Eurydice. (2018). National System of Education: Austria. Retrieved from https://eacea. ec.europa.eu/national-policies/eurydice/content/austria_en.

Fend, H. (2001). Bildungspolitische Optionen für die Zukunft des Bildungswesens. Zeitschrift für Pädagogik, 43, 37-48.

Fend, H. (2018). Bildungsforschung und Schulentwicklung in Österreich. In H.Altrichter, B. Hanfstingl, K. Krainer, M. Krainz-Dürr, E. Messner, \& J. Thonhauser (eds.), Baustellen [in] der österreichischen Bildungslandschaft (pp. 14-25). Münster:Waxmann.

Gamsjäger, M., Altrichter, H., \& Steiner, R. (2019). Wirkungen und Wirkungswege einer Bildungsstandards-Reform: Die Sichtweise von Lehrpersonen und Schulleitungen in österreichischen Primarschulen. Zeitschrift für Bildungsforschung. 9(2), 139-158. DOI: 10.1007/s35834-019-00239-1.

Gewirtz, S., Mahony, P., Hextall, I., \& Cribb, A. (2009). Policy, Professionalism and Practice: Understanding and Enhancing Teachers' Work (pp. 3-16). In: S. Gewirtz, P. Mahony, I. Hextall, \& A. Cribb (Eds.), Changing Teacher Professionalism: International Trends, Challenges, and Ways Forward. Oxon: Routledge.

Gulati, R. (1995). Does familiarity breed trust? The implications of repeated ties for contractual choice in alliances. Academy of Management Journal, 38, 85-112.

Gustafsson, J.-E., Ehren, M. C. M., Conyngham, G., McNamara, G., Altrichter, H., \& O’Hara, J. (2015). From inspection to quality. Studies in Educational Evaluation, 47, 47-57.

Heinrich, M. (2018). Does dialogue work? Governance Analysen zur Notwendigkeit eines, dialogic turn' evidenzorientierter Steuerung am Beispiel der Schulinspektion (pp. 323-334). In: K. Drossel, \& W. Eickelmann (Eds.), Does, What Works' Work? Bildungspolitik, Bildungsadministration und Bildungsforschung im Dialog. Münster: Waxmann.

Honingh, M., Ehren, M., \& van Montfort, C. (2018). Het vernieuwde toezicht in het onderwijs. Hooggespannen verwachtingen van kwaliteitszorg, bestuurskracht en maatwerk. Bestuursunde, 27(4), 19-29. DOI: 10.5553/Bk/092733872018027004002.

Hult, A. \& Edström, C. (2016). Teacher ambivalence towards school evaluation: promoting and ruining teacher professionalism. Education Inquiry, 7(3), 305-325.

Kemethofer, D., \& Altrichter, H. (2015). Schulqualität Allgemeinbildung (SQA) in der Einschätzung von Schulleitungen. Erziehung und Unterricht, 165(7-8), 675-690.

Kotthoff, H.-G., Böttcher, W., \& Nikel, J. (2016). Die, Schulinspektion' zwischen Wirkungshoffnungen und Wirksamkeit (pp. 325-359). In: H. Altrichter, \& K. Maag Merki (Eds.), Handbuch Neue Steuerung im Schulsystem. Wiesbaden: SpringerVS.

Lassnigg, L., \& Vogtenhuber, S. (2015). Challenges in Austrian educational governance revisited. (IHS Sociological Series, No. 107). Vienna: IHS.

Maier, U., \& Kuper, H. (2012).Vergleichsarbeiten als Instrumente der Qualitätsentwicklung an Schulen. Die Deutsche Schule, 104(1), 88-99.

Maritzen, N. (2015). SQA - Ein Blick von außen. Erziehung und Unterricht, 165(1-2), 12-18.

Maritzen, N. (2018) Was heißt und zu welchem Ende studiert man Daten? In K. Drossel \& B. Eickelmann (eds.), Does, What works' work? (pp. 37-54). Münster:Waxmann.

Maroy, C., \& van Zanten, A. (2009). Regulation and competition among schools in six European localities. Sociologie du Travail, 51(S1), e67-e79.

Nairz-Wirth, E., \& Feldmann, K. (2019). Teacher professionalism in a double field structure. British Journal of Sociology of Education. 40(6), 795-808. DOI: 10.1080/01425692. 2019.1597681.

Pavolini, E. (2015). Marketization and Managerialization of Education Policies in a Comparative Perspective (pp. 73-96). In: T. Klenk, \& E. Pavolini (Eds.), Restructuring Welfare Governance. Cheltenham: Edward Elgar. 
Piezunka, A. (2019). Struggle for acceptance - Maintaining external school evaluation as an institution in Germany. Historical Social Research, 44(2), 270-287.

Posch, P. \& Altrichter, H. (1993). Schulautonomie in Österreich.Vienna: BMUK.

Radnitzky, E. (2015). SQA - ein Generationenprojekt. Erziehung und Unterricht, 165(1-2), 8-11.

Rauscher, E. (1999). Schul-Autonomie-Handbuch.Vienna: BMUK.

Rürup, M. (2008). Typen der Schulinspektion in den deutschen Bundesländern. Die Deutsche Schule, 100(4), 467-477.

Schratz, M. \& Hartmann, M. (2009) Schulautonomie in Österreich: Bilanz und Perspektiven für eine eigenverantwortliche Schule. In W. Specht (ed.), Nationaler Bildungsbericht Österreich 2009. Band 2 (pp. 323-340). Graz: Leykam.

Skliris, B., Petrovic, A., Klimann, T., \& Svecnik, E. (2018). Evaluation der Initiative SQA. Graz: BIFIE.

Sowada, M. G., \& Dedering, K. (2016). Die Reform der Reform (pp. 169-199). In: Arbeitsgruppe Schulinspektion (Eds.), Schulinspektion als Steuerungsimpuls?. Wiesbaden: SpringerVS.

Tillmann, K.-J., Dedering, K., Kneuper, D., Kuhlmann, C. \& Nessel, I. (2008) PISA als bildungspolitisches Ereignis. Wiesbaden: SpringerVS.

Tonne, G. H. (2018).Vorwort. Schulverwaltungsblatt für Niedersachsen, 3, 109.

West, A., \& Nikolai, R. (2013). Welfare regimes and education regimes. Journal of Social Policy, 42(3), 469-493.

Windzio, M., Sackmann, R., \& Martens, K. (2005). Types of Governance in Education: A Quantitative Analysis. Retrieved from https://www.econstor.eu/bitstream/10419/ 28275/1/501321926.PDF.

Young, G. (2003). Contextualizing Cooperation (pp. 77-19). In: M. A. West, D. Tjosvold, \& K. G. Smith (Eds.), International Handbook of Organizational Teamwork and Cooperative Working. Chichester: Wiley. 


\title{
8
}

\section{HIERARCHICAL STRUCTURES WITH NETWORKS FOR ACCOUNTABILITY AND CAPACITY BUILDING IN SINGAPORE}

\section{An evolutionary approach}

\author{
Shu-Shing Lee, Jeanne Ho and Tay Lee Yong
}

\section{Introduction}

Singapore was first pushed into the limelight when the 2012 Programme for International Student Assessment (PISA) results were announced and Singapore was amongst the top five performers for mathematics (second), reading (third) and science (third) (OECD, 2016). This stellar performance did not happen overnight, and Singapore has steadily made progress. Goodwin et al. (2017) reported that in the 2013 Trends in International Mathematics and Science Study, Singaporean students topped rankings in mathematics and science achievements. Around 90\% of the student population who took the test achieved scores above the international median score. Singapore's achievement as a high-performing system was consistently validated in the 2009 PISA, 2012 PISA, 2015 PISA and 2018 PISA. In 2015 PISA, the Organisation for Economic Cooperation and Development (OECD) ranked Singapore as the top education system in the world. Hong Kong was ranked in the second place and South Korea in the third place (Coughlan, 2015). In 2018 PISA, Singapore continued to be amongst the top education systems coming in the second in English, mathematics and science after China. Although Singapore came in the second in 2018, Singapore students did significantly better in literacy skills than in the 2015 PISA, and they also performed better than the OECD average in higher order thinking skills (The Straits Times, 2019).

Singapore is a small city-state, which is two-thirds the size of New York City and one-tenth the size of Shanghai. The scale of Singapore's education system could be one reason why it is possible for the Singapore Ministry of Education (MOE) to achieve its current status in a relatively short period of time even though Singapore is a young nation that achieved independence in 1965. Internationally, there is growing interest in high-performing education systems, and there has been a trend 
towards global policy borrowing for system reform. Scholars, such as Earley and Greany (2017) as well as Darling-Hammond et al. (2017), stress that each context has its unique nuances. While there is interest to understand the factors contributing to Singapore's good performance, in reality, there is likely a combination of factors at play and careful planning and deliberate modifications over the years.

In this chapter, we leverage existing literature and the authors' collective insights of Singapore's education context as a researcher, school leader and head of department (HOD), respectively. We take an evolutionary perspective to unpack the trajectory of the Singapore education system. The system's structures stem from hierarchical control and evolved to include network governance. Throughout Singapore's development as an education system, the MOE has continuously made efforts to build the capacities of teachers and leaders, initially, adopting more centralised approaches of capacity building to enact a standardised curriculum and later developing capacities for school-led innovations through network structures. We argue that MOE's capacity-building efforts have increased the competencies of teachers and leaders over time and, subsequently, enabled MOE to trust schools. Increasing trust in schools further enabled a shift from a centralised approach of accountability towards school-led accountability and innovations. In other words, schools and teachers have developed sufficient capacities so MOE trusts that schools can be empowered to implement school-led accountability and innovations. Figure 8.1 broadly summarises the progression of accountability, capacity building and trust in the Singapore education system.
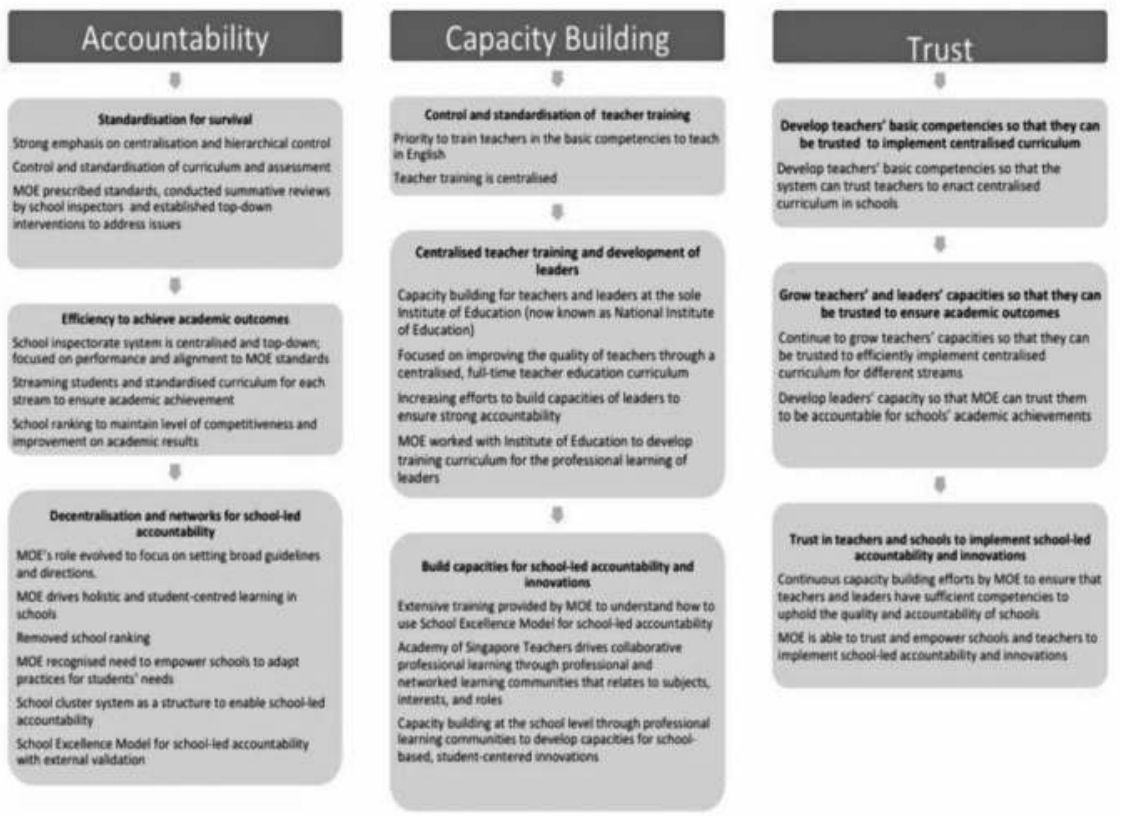

FIGURE 8.1 Progression of accountability, capacity building and trust in the Singapore education system 
In the sequent sections, we elaborate from an evolutionary lens how Singapore has evolved structures and efforts related to accountability, trust and capacity building to achieve the most important objective for education in Singapore: 'to educate a child to bring out his greatest potential, so that he will grow up into a good man and useful citizen' (Former Prime Minister Lee Kuan Yew, in Goh, 1979, p. iii).

\section{Hierarchical governance and capacity building for standardisation and efficiency \\ Standardisation to ensure the survival of a nation (1959-1978)}

During the 1960s, the Singapore education system began as a mix of communitybased schools that implemented different curricula. The mediums of instruction involved different languages, namely, English, Chinese, Tamil and Malay. To ensure the survival of a young nation, with no natural resources, there was an urgent need to ensure the education system developed skilled workers that attracted global multinational corporations. There was a rapid move towards developing a national system of education with a strong emphasis on centralisation and hierarchical control. English was chosen as the medium of instruction (Gopinathan et al., 2008). Centralised resources were allocated to build and expand new schools rapidly.

Priority was placed on capacity building for teachers. There was a pressing need to (1) recruit more teachers and (2) to train new teachers and retrain existing teachers to teach in English. The focus of capacity building was to regulate teacher training and develop well-qualified teachers who could implement standardised curriculum and assessment.

Accountability of schools was also centralised. MOE prescribed standards that schools needed to meet. Accountability and school appraisal were done by the school inspector focusing on financial management, implementation of MOE's policies and school's academic performance. MOE conducted external, summative reviews on an annual basis to identify issues and establish top-down interventions to address any issues (Ho and Koh, 2018).

Overall, accountability and capacity building during this survival period (1959-1978) focused on, and was designed to support, standardisation ( $\mathrm{Ng}$, 2008). The goal was to develop an education system that was accountable to standards set by MOE. There were urgent demands to expand and build schools as well as recruit and train more teachers so that more students could be educated to drive Singapore's nation building and economic development. Teachers were trained through a centralised teacher training institute to develop basic competencies so that they could implement MOE's standardised curriculum. MOE kept schools accountable through yearly evaluations and intervened accordingly to ensure standards were met. 


\section{Efficiency to reduce educational wastage and achieve academic outcomes (1979-1996)}

In the earlier years, between 1959 and 1978, MOE was able to ensure the basic quality and competencies of schools and teachers by adopting a standardised approach. The rapid expansion of schools and training of teachers enabled the system to achieve high student enrolment, but it also resulted in education wastage. There were high dropout rates (Ho and Koh, 2018). Accordingly, MOE's concerns with accountability and capacity building shifted to develop an approach that would reduce dropout rates. Subsequent changes included streaming of students according to academic performance with a standardised curriculum for each stream (Gopinathan et al., 2008). Teacher capacity building was similarly restructured: moving from part-time courses into full-time courses at one Institute of Education (now known as the National Institute of Education). The key thrust of capacity building was no longer about having enough teachers but shifted to improving the quality of teachers through a centralised, full-time teacher education curriculum so teachers developed competencies to enact the standardised curriculum for each stream of students. Teacher learning and student learning were nurtured through a centrally designed curriculum (Goh and Lee, 2008).

Accountability of schools continued to be centralised and top-down but became more structured and aligned to emphasise performance and alignment to MOE's standards ( $\mathrm{Ng}, 2008$ ). The most profound change was the move from the appraisal of schools by an individual school inspector to a school inspectorate system where a group of inspectors would evaluate each school every 4-5 years on four aspects, management of school, instruction, extracurricular activities and student welfare (Wee and Chong, 1994).

Another significant development was the introduction of school ranking in 1992 as a form of local accountability ( $\mathrm{Ng}, 2007)$. School rankings were published in the press to include academic results and the ability of schools to value-add to students' improvement in academic results from the time they entered the secondary school to the point of their graduation. School ranking became a tool for local accountability. It created a level of competitiveness amongst schools to ensure students' academic achievements. Schools that ranked poorly got a wake-up call to find ways to improve students' academic outcomes.

MOE recognised the complexity of such local accountability, particularly where schools required further capacity and a strong middle management team to improve their performance on these rankings. As a result, MOE created the position of HODs in 1983, together with a full-time management programme to build HODs' capacities to perform this role (Lim-Chan, 2006). These HODs were teachers but also tasked with leadership and administrative support within the school. The introduction of HODs provided a way for the system to identify good teachers to be promoted and trained to be school principals. To further support administrative management and accountability within the school, 
vice principals were assigned to schools. Thus, school principals were supported by vice principals and HODs. MOE also began to work with the Institute of Education (now known as the National Institute of Education) to develop a curriculum for the professional learning of vice principals and principals to nurture their competencies for managing schools effectively. In this way, MOE emphasised capacity building to develop a strong school management team so that schools have the competencies to be trusted and accountable to achieve students' academic outcomes. Stronger middle management in schools was expected to support local accountability and ensure there was strong accountability throughout the education system.

The education system remained centralised but the focus was now less about levelling up the capacities of teachers and students to ensure the survival of a young nation, but instead on creating a centralised system that could function efficiently to reduce education wastage. Students were streamed and provided a standardised education experience that was based on their academic ability. The school inspectorate system focused on academic performance and adherence to MOE's standards. Schools were ranked according to academic outcomes to maintain a level of competitiveness and focus on academic improvement.

However, the development of a strong middle management team in schools could be seen as initial signals that the system was preparing for shifts towards school-led accountability (Ng, 2007). MOE's capacity-building efforts in developing quality teachers and a strong middle management team allowed it to devolve more responsibilities and accountability downwards locally to schools. Developing the competencies of teachers and leaders through one centralised approach at a single Institute of Education allowed the MOE to trust that schools had the capacity and would take the responsibility to implement the centralised curriculum for different streams of students. MOE's trust in schools was also indicated by the change in the school appraisal system, from a sole inspector whose sole judgement and views were binding to an inspection by a group of inspectors who allowed for more discussion and a variety of perspectives and input, focused on developing schools (Wee and Chong, 1990).

\section{Hierarchical with network governance for school-led accountability and capacity building (1997 onwards)}

\section{Cluster of schools as a structure for hierarchical with network governance}

The increasing global development and drive towards knowledge-based industries in the 21st century meant that for Singapore to remain competitive, it was insufficient to focus solely on students' academic outcomes. MOE felt a need to develop students with thinking dispositions and skills to meet the needs of a knowledge-based economy and, as result, introduced the Thinking Schools, Learning Nation (TSLN) vision in 1997 (Goh, 1997). This vision articulated a 
more inclusive, student-centred and flexible approach to teaching and learning, recognising more diverse ways of thinking, learning and innovative pedagogies (Hogan and Gopinathan, 2008). Such change also required MOE to change its position and coordination of school quality. Teo $(2000 \mathrm{a}, \mathrm{b})$, the then Minister for Education, shared that MOE's role was to set the broad directions and guidelines and not about getting schools to conform to detailed rules and procedures. The intention was to encourage more diversity and innovation at the school and teacher levels, with 'ideas bubbling up through the system rather than being pushed down from the top' (Shanmugaratnam, 2005). This development aligned with earlier efforts at building the capacities of a strong school management team to enable school-led accountability. Furthermore, the system made progression by introducing the school cluster system to create opportunities for collaboration, professional development and sharing of resources between schools.

The school cluster system was introduced in 1997 to support the TSLN vision as a structure for coordinating capacity-building efforts and encouraging individual school innovations while ensuring schools align with the overall directives set by MOE. As shown in Figure 8.2 in the cluster system, MOE sets policies and the national curriculum; schools are organised in four zones (north, south, east and west), where each zone constitutes seven to eight clusters. Each cluster includes 10-13 schools which are overseen and supported by a cluster superintendent (The Ministry of Education, Singapore, 2019a).

The school cluster system provides a unique structure to combine hierarchical steering and accountability with checks and balances towards a centralised framework with a more decentralised structure of network governance and collaboration. In the hierarchical structure, cluster superintendents are senior or experienced principals whose role is to communicate MOE's policies and to guide and supervise school leadership teams to understand and implement MOE's policies. Cluster superintendents monitor the implementation of MOE's policies in their cluster of schools by collecting data and feedback from their schools on a centralised framework, ensuring consistency in school appraisal

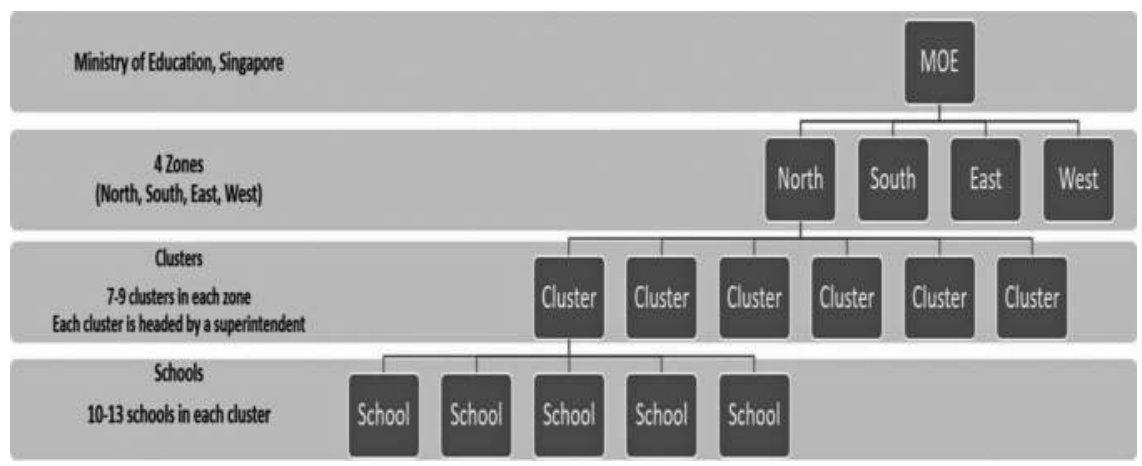

FIGURE 8.2 The school cluster system 
(Chew et al., 2003); they are the line manager of their schools' principals who report to them and are appraised and mentored by them (Ho and Koh, 2018). The school cluster system also aims to improve hierarchical steering by providing a clear communication structure whereby cluster superintendents, who understand the capacity-building needs of their schools, can initiate customised capacity-building efforts to help schools develop competencies to implement national policies and remain accountable to MOE.

The school cluster system is also a strategy for MOE to build relationships with schools and ensure collaboration between schools as part of a more decentralised structure of network governance. Cluster superintendents play a key role in facilitating the collaboration across the schools within their cluster and to help schools make sense of policies collectively, share resources and good practices (Teo, 2000a). Under the guidance of cluster superintendents, schools are trusted to be innovative and creative in providing education experiences that align with national policy but equally benefit and are fit for the students and teachers in their local contexts (Teo, 1998). The school cluster system promotes professional learning across schools by tapping on expertise and resources of schools within the same cluster. The school cluster system is a system-level structure that devolves greater school autonomy and trust in schools by creating a culture of collaborative decision-making and capacity building for innovations.

\section{Network governance through school-led appraisal and accountability}

As schools were given greater autonomy in the introduction of the school cluster system, MOE argued that the centralised model of accountability was no longer fit for purpose (Teo, 1997). In response, MOE introduced the School Excellence Model (SEM) in 2000 to task schools with their own quality assurance while removing centralised control. The shift from central to more local forms of accountability aimed to promote and acknowledge diversity and innovation in schools (Ng, 2013). School management teams were trained to self-evaluate the quality of their school using a common framework, where these self-evaluations would in turn be validated by a team of external appraisers once every 5 years.

The development of SEM shows how deliberate efforts were made to empower schools to take charge of their own appraisal and to support school-led accountability. The SEM provides broad standards and performance indicators that go beyond academic outcomes to emphasise students' holistic development. Schools are required to review processes (enablers) and outcomes (results) using an expanded concept of student outcomes to include the physical, social, aesthetics, moral and emotional development of the child (Teo, 1999). SEM does not dictate a prescribed approach for school evaluation but allows for variations in how schools develop capacities for innovations and achieve excellence (Teo, 2002). Even though MOE sets out key performance areas and sample indicators in SEM, schools can decide on how they want to achieve excellence in these 
areas and can design their own self-evaluation ( $\mathrm{Ng}, 2008$; Seah and Ow, 2003). The external appraisers' role is to assist schools in calibrating the school's selfassessment and identification of its strengths and areas for improvement (Seah and Ow, 2003). The approach marks a clear shift in Singapore's education system from a summative and external approach to school evaluation and accountability to a formative one with the intention of schools owning their self-assessment and charting their own approach towards school improvement.

The shift in approach signals a level of trust by MOE in schools to objectively and accurately self-evaluate their performance; trust that was developed by establishing a strong school management team, including the principal, vice principals, HODs and school staff developer, providing the management team with extensive training in the SEM and ensuring they have the capacity to lead and monitor their school (in both instructional, curriculum and administrative areas). In particular, the training of school principals in SEM is important as leaders are trained to understand that SEM is designed to include broad standards, key performance areas and sample indicators beyond academic results. School leaders are trained to understand that there are various approaches to achieving outcomes.

\section{Evolution of SEM and understandings of broader educational outcomes}

As with everything in the Singapore education system, we are constantly reviewing and fine-tuning policies. SEM has evolved to a stage whereby schools choose which key areas (such as staff development and well-being, partnerships, teaching and learning) they would want the external assessors to validate, moving towards a customised validation (The Ministry of Education, Singapore, 2019b). To reduce the effort spent on paper work, so that schools can focus on the self-evaluation process instead of the end product of the evaluation report, SEM has been modified over time to be less onerous and to help schools focus on the substance and not the form of SEM (Ng, 2003).

Changes in the model over time have included the removal of school rankings to support the broadening of student outcomes beyond academic performance and the flexibility to meet different students' needs as stated in the TSLN vision (The Straits Times, 2015). The intention is on building a school culture of holistic education where each school has its own niche and peak of excellence (Heng, 2012b, 2013, 2014b). The main principle throughout the implementation of SEM is one of MOE investing in capacity building of schools which would allow MOE to trust that all schools are providing high-quality education and can cater to the needs of their unique student population (Heng, 2012a).

The past model of hierarchical governance for standardisation and efficiency to achieve academic outcomes has, however, ingrained a clear set of expectations and habits in parents and teachers which have not yet changed, despite the various measures to move towards a more holistic education system and a broader 
set of education outcomes (e.g. by removing school rankings, and communicating to schools and teachers the need to focus on 'nurturing of the whole child', Ministry of Education, 2012). Studies like the Core Research Programme, for example, indicate that the teachers' focus remains on teaching content and knowledge to pass exams, despite attempts to get teachers to explore different pedagogies (Hogan, 2014). Parents also still predominantly push their children to do well in academic subjects and on tests; parents expect and hold schools and teachers accountable for academic achievement. As a result, schools still feel primarily accountable to deliver such results.

\section{School-led capacity building for innovations}

To address teachers' competencies for a more holistic, student-centred and flexible approach (instead of delivering a standardised central curriculum), MOE established the Academy of Singapore Teachers (AST). AST aims to support professional learning by bringing together teachers from different schools. Its mission is to build a teacher-led culture of professional learning and excellence centred on the holistic development of the child as well as the growth of the teacher to bring about school success (Academy of Singapore Teachers, n.d.). Broadly, AST encourages two approaches of collaborative capacity building where teachers inquire about their practice in (1) professional learning communities (PLCs) at the individual school level and (2) networked learning communities (NLCs) across schools.

\section{Capacity building through professional learning communities in schools}

These two learning communities, as promoted by AST on behalf of MOE, intend to capacitate teachers to take ownership over their teaching and to involve schools in supporting teachers' professional learning. The centralised (top-down) promotion of PLCs is part of MOE's move to indicate that the system trusts that teachers already have basic competencies, where MOE encourages them to take greater autonomy, reflect, inquire and innovate their practices (Shanmugaratnam, 2005). AST (MOE) provides them with 'three big ideas' of what to achieve as part of the PLCs: (1) ensuring students learn, (2) building a culture of collaboration and (3) focusing on student learning outcomes (DuFour, 2004) with an additional set of four critical questions on student learning to facilitate professional conversations (DuFour and Fullan, 2013):

1. What is it we expect students to learn?

2. How will we know when they have learned it?

3. How will we respond when they don't learn?

4. How will we respond when they already know it? 
AST also recommends that schools provide at least 1 hour during curriculum time for the PLCs to meet, known as timetabled time (Shanmugaratnam, 2005), where teachers in the same grade level and/or who teach the same content subject meet. Schools are expected to support the PLCs by creating 'white space' during curriculum hours so that the PLCs have protected time to meet, dialogue on student learning issues and develop teaching resources where relevant. PLCs are also supported by a coalition team who are part of the school management team, comprising the principal, vice principal and school staff developer. This team provides the structures, processes, resources and culture to support teacher learning within the school's PLC framework (Hairon et al., 2015).

PLCs are MOE's way of building schools' and teachers' capacities to initiate school-led innovations, in order to achieve students' academic outcomes, holistic student development and address the needs of specific school and classroom contexts (Hairon and Tan, 2017). Capacity-building efforts are key so that schools and teachers develop the capabilities to take ownership of and accountability for developing school-based curriculum innovations. This is important because even in a small system like Singapore, with the progression towards student-centred learning experiences, the system can no longer adopt a one-size-fits-all approach of providing centralised curriculum or translating curriculum models for every school and expecting schools to enact it. Capacity building is essential, so schools have the abilities to understand the overall curriculum directions that MOE is encouraging, and for every school to translate these directions into their own school-based curriculum that is suitable for local needs. In fact, the commitment from MOE to encourage PLCs in schools aligns with the idea of every school a 'thinking school' that is articulated in the TSLN vision in 1997 which underpinned the initial SEM. PLC is an example of collaborative and community forms of professional development where efforts are on building camaraderie amongst teachers and their capacities to interrogate practices (Hairon and Dimmock, 2012). Today, almost all schools in Singapore have adopted PLCs, with variations in the inquiry approaches chosen, including Lesson Study, Action Research and Learning Study (Lee and Lee, 2013).

\section{Capacity building through networked learning communities across schools}

In addition to PLCs within schools, MOE has also encouraged NLCs across schools. While PLCs stress professional learning in communities of teachers within schools, NLCs involves teachers from different schools, promoting teachers to learn from one other, learn with one another and learn on behalf of each other (Jackson and Temperley, 2007). These NLCs may be (1) subject based, like subject chapters under the AST; (2) role based, like an NLC for School Staff Developers and (3) interest based NLCs 'on any area that anyone is passionate about' (Heng, 2014a). 
Subject chapters, as the name implies, are organised by subject disciplines (e.g. chemistry, geography and mathematics) to advance the pedagogies and instructional practice of subject teachers. All subject teachers are members of their respective subject chapters, but attendance at subject chapter meetings is voluntary in nature. Interest based NLCs cater to teachers with specific interests, such as the Normal Course NLC of teachers who share a common passion to learn effective pedagogical practices to implement the Normal Course for lower progressing students. An example of a role-based NLC is the School Staff Developer NLC, which involves School Staff Developers from different schools where they dialogue about how to proactively understand the professional development needs of teachers in their schools and how to facilitate professional learning by matching teachers' needs to the right professional development opportunities.

As NLCs involve teachers across schools, it can function at the cluster, zonal or even national levels, such as an NLC on Character and Citizenship Education. An example of a cluster/zonal NLC could be when cluster superintendents identify an area of learning amongst schools under their charge, for example differentiated instruction. Then teachers from these schools could be organised to learn how to design different learning experiences for differentiated instruction to meet their students' needs and teachers' readiness.

Jackson and Temperley (2007) contend that professional learning within and across school communities is necessary for rich competence development as communities within a school have too limited a scale to provide sufficient scope for teacher professional learning. NLCs add opportunities for the development to school-based PLCs by extending the breadth of diverse teaching and learning experiences from teachers across a range of school contexts. These capacity-building efforts ensure teachers and schools continue to develop capacities to uphold the quality and accountability of schools as indicated in the SEM. Capacity building in PLCs and NLCs also means that schools and teachers know where to seek expertise within and across schools when they face challenges. As we have argued throughout the chapter, the structure of centralised steering combined with localised collaboration and accountability through a school cluster structure, a strong emphasis on capacity building, sharing of resources and practices allows MOE to trust schools to implement school-led accountability within a framework of school excellence and initiate practices and pedagogies to improve the performance of students in their local contexts. Figure 8.3 summarises evolution of the Singapore education system which sets the context for planned efforts to develop accountability, trust and capacity in the system.

\section{The next leap: Capacity building in communities and networks for continuous improvement}

As the previous section explained, hierarchical with network governance sets the context for relationships amongst capacity, accountability and trust in the Singapore education system. As shown in Figure 8.4, capacity building is a key 


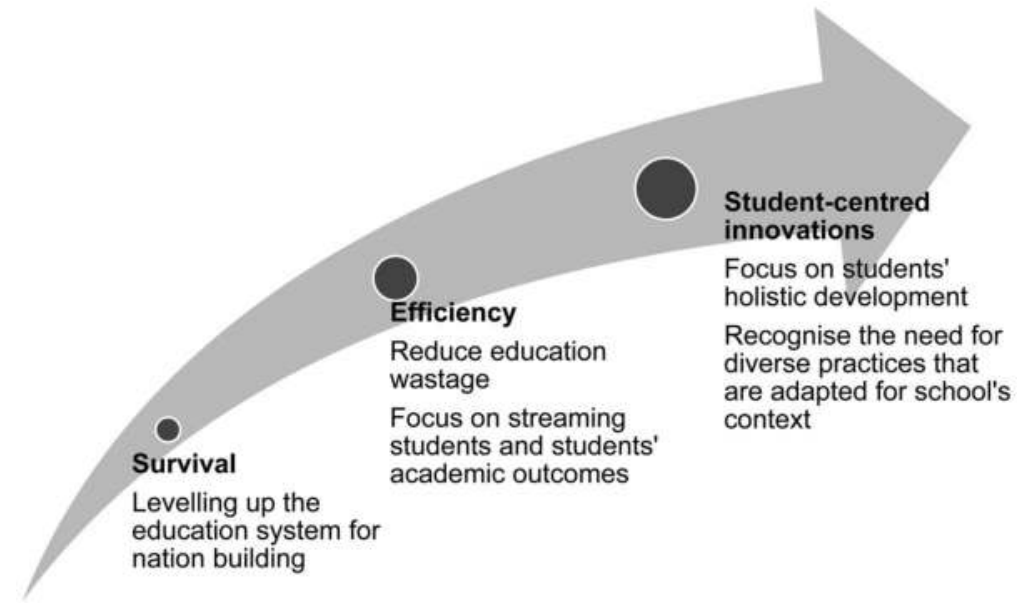

FIGURE 8.3 Evolution of the Singapore education system

pillar in the system to allow MOE to trust teachers as professionals and empower them to design quality learning experiences for students. Capacity-building efforts also develop leaders' competencies and understandings of SEM so leaders can be trusted to engage in self-appraisal and innovations.

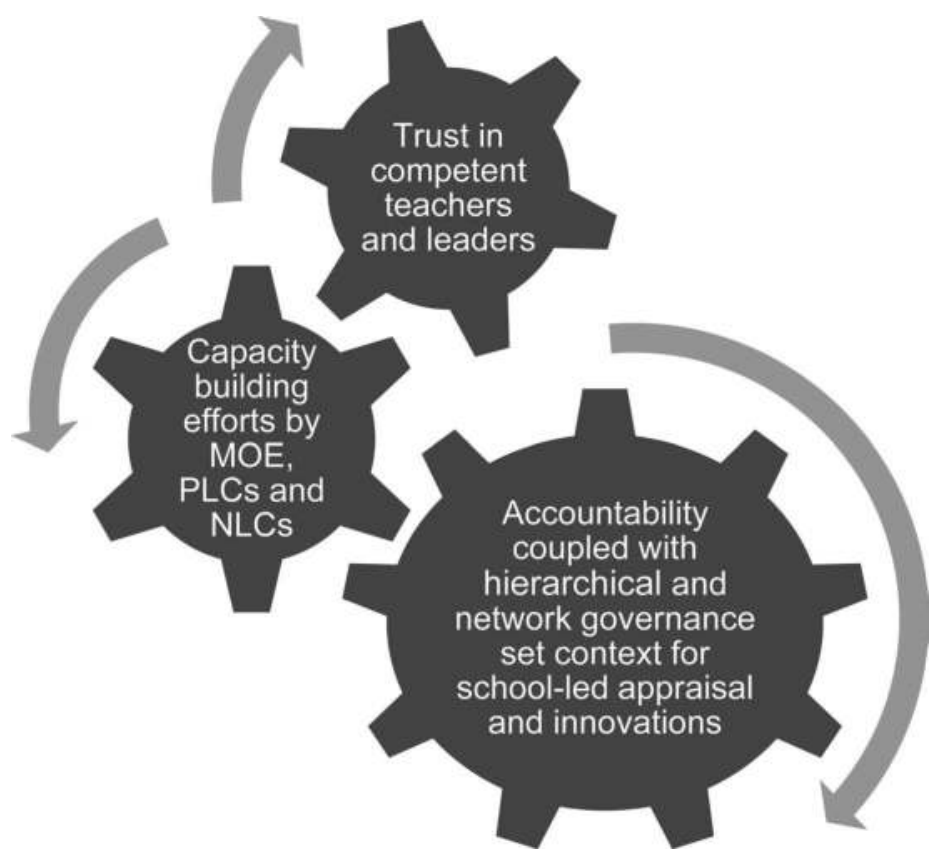

FIGURE 8.4 Relationships amongst accountability, capacity building and trust 
An important next step for the system to move forwards is further capacity building in NLCs and PLCs and enabling teachers to take ownership of their professional learning and develop competencies that are relevant to their particular contexts. The successful implementation of PLCs and NLCs likely hinges on perseverance and leadership to overcome cultural and workplace practices that are tied to a hierarchical education system that is working to include network governance. Existing cultural practices that may pose challenges for PLCs and NLCs in schools include teachers' view that participating in these learning communities is a burden on top of their already high workloads, teachers' reluctance to change established teaching practices for fear of compromising students' test scores and a dependency culture where teachers expect top-down guidance for implementing PLCs and NLCs. The culture of collaboration and learning in learning communities can be nurtured over time by taking a continuous, developmental perspective. Leaders play key roles in providing the structural and cultural support to develop teachers' will to learn and be agents of their professional learning (Hairon and Dimmock, 2012; Hairon and Tan, 2017). The approach to PLC and NLC implementation needs to allow teachers to provide feedback to leaders either informally or formally. It is useful for leaders to seek feedback as it allows teachers' voices and needs to be heard and for leaders to make conscious efforts towards refining the ways PLCs are implemented in schools (Ho et al., 2019), as well as for NLCs to function across schools.

The introduction of NLCs in Singapore is part of an international trend of 'self-improving school systems'. The rationale is that a hierarchical approach to bring about improvements is no longer sufficient. Instead, schools need to improve quality and strive for excellence at the local, school-led level (Greany, 2017; Hargreaves and Shirley, 2009). In Singapore, PLCs work on collaborative teacher learning and influencing practices at the school level while NLCs and the school cluster system establish networks across schools to facilitate the sharing and propagating of good practices between schools in the system. NLCs and the school cluster system support networked professional learning and school improvement activities as teachers and leaders collaborate with other schools to ensure that their learning and innovations align with policies and goals (Hairon and Dimmock, 2012).

Lave and Wenger (1991) also suggest that communities provide spaces for cultivating different perspectives. In Singapore, NLCs and the school cluster system enable teachers to work with their peers in other schools to understand different possibilities and practices for improving student outcomes. MOE is moving away from a hierarchical, one-size-fits-all approach towards a facilitating role that leverages networks and communities to build capacity and generate innovative solutions to complex problems. MOE still bears responsibility to ensure accountability and standards are met to drive the system towards positive national outcomes. However, national outcomes have been broadened to include more than just academic performance and to embrace school-specific outcomes as well as students' holistic development. MOE's consistent emphasis on capacity 
building ensures that schools have the competencies to customise practices and innovate to meet students' needs. Competent schools enable the system to empower schools to decide how they want to improve practices while holding them accountable for aligning practices and performances to policies. This is an increasing tenet of international thinking on system reform and improvement (Greany, 2017; Hargreaves and Fullan, 2015) where school leaders are expected to take a greater role in unpacking and explaining what policies mean to teachers and students. Teachers and leaders need to be reflective and interpret policies for the school context.

Another tenet of international thinking, which aligns with our school cluster system and NLCs, relates to how networks and collaborations involve trusting schools and teachers to leverage lateral networks for continuous learning and improvement (Greany, 2017; Hargreaves and Fullan, 2015). Giving schools and teachers opportunities to develop a school-based curriculum and implement improvements within a broader set of guidelines is expected to bring about more sustainable reform. The underlying premise is one of developing teacher professionalism through teacher-led professional learning in communities and networks and allowing them to self-regulate through responsible professional dialogue (Hargreaves and Shirley, 2009). Singapore similarly operates on the philosophy of 'teachers for teachers' (Academy of Singapore Teachers, n.d.) where teachers are expected to take ownership in pursuing quality practices and learn together.

Hargreaves and Fullan (2015) state that the building blocks of a self-improving system include clusters of schools, local solutions, co-construction and system leaders. We envisage that Singapore is well positioned to be a self-improving system because our system is organised to create opportunities for schools to work laterally and for school leaders to 'lead nationally' (Heng, 2014a,b). Our system adopts both hierarchical and network governance where schools are trusted and thus empowered to adopt localised solutions and innovations. The cluster system builds joint professional capital (Hargreaves and Shirley, 2009) so teachers across schools develop a shared sense of working towards providing students with holistic learning experiences. Porritt et al. (2017) similarly state that joint practice development in communities enables teachers to learn new practices through mutual engagement and collaboration. For Singapore, this joint practice development extends laterally beyond schools. The school cluster system continues to embed opportunities for collaboration to maintain and uphold the quality of education in Singapore.

\section{Conclusion}

This chapter adopted an evolutionary lens to unpack the Singapore education system. Within it, we have described how the system stems from hierarchical governance but is progressing to be one that includes hierarchical and network governance. With this context in mind, we have described the deliberate efforts 
made by the system to evolve accountability structures alongside the capacity building of teachers and leaders. Initially, the system adopted more centralised approaches of capacity building to enact standardised curriculum, later developing capacities for school-led innovations through network structures. The quality and competencies of teachers and leaders are key so that the system trusts schools to provide quality and holistic learning experiences for students.

Capacity building continues to be a key aim, as the system works towards a self-improving system. A self-improving system involves developing teachers' and leaders' competencies within and across school communities and networks. Communities are platforms to develop teachers' professionalism and ownership of their own learning. Networks allow teachers to see new perspectives, develop trust amongst peers and build synergies across schools. While Singapore has come a long way, the country continues its journey as a system that is constantly learning and improving.

\section{References}

Academy of Singapore Teachers. (n.d.). Our Mission and Vision. Retrieved from https:// www.academyofsingaporeteachers.moe.gov.sg/about-ast/our-mission-and-vision.

Chew, J., Stott, K., \& Boon, Z. (2003). On Singapore:The making of secondary school principals. International Studies in Educational Administration, 31(2), 54-75.

Coughlan, S. (2015, May 13). Asia tops biggest global school rankings. BBC News. 48(4), 635-650. Retrieved from: http://bbc.com/news/business-32608772.

Darling-Hammond, L., Burns, D., Campbell, C., Goodwin, A. L., Hammerness, K., Low, E. L., \& Zeichner, K. (2017). Empowered Educators: How High-Performing Systems Shape Teaching Quality Around the World. John Wiley \& Sons.

DuFour, R. (2004). What is a 'professional learning community'?. Educational Leadership, 61(8), 6-11.

DuFour, R., \& Fullan, M. (2013). Cultures Built to Last: Systemic PLCs at Work. Solution Tree Press.

Earley, P., \& Greany,T. (2017). Postscript:The Future of Leadership (pp. 222-228). In: P. Earley, \& T. Greany (Eds.), School Leadership and Education System Reform. London: Bloomsbury.

Goh, C. B., \& Lee, S. K. (2008). Making Teacher Education Responsive and Relevant (pp. 96-113). In: S. K. Lee, C. B. Goh, B. Fredriksen, \& J. P. Tan (Eds.), Toward a Better Future; Education and Training for Economic Development in Singapore Since 1965. Washington, DC: The World Bank.

Goh, C. T. (1997). Speech by Prime Minister Goh Chok Tong at the opening of the 7th International Conference on Thinking on Monday 2 June 1997, at 9 a.m. at the Suntec City Convention Centre Ballroom. Retrieved from https://www.moe.gov.sg/media/ speeches/1997/020697.htm.

Goh, K. S. (1979). Report on the Ministry of Education 1978. Singapore National Printers.

Goodwin, A. L., Low, E. L., \& Darling-Hammond, L. (2017). Empowered Educators in Singapore: How High-Performing Systems Shape Teaching Quality. John Wiley \& Sons.

Gopinathan, S., Wong, B., \& Tang, N. (2008). The evolution of school leadership policy and practice in Singapore: Responses to changing socio-economic and political contexts (insurgents, implementers, innovators). Journal of Educational Administration and History, 40(3), 235-249. 
Greany, T. (2017). Collaboration, Partnerships and System Leadership Across Schools (pp. 56-65). In: P. Earley, \& T. Greany (Eds.), School Leadership and Education System Reform. London: Bloomsbury.

Hairon, S., \& Dimmock, C. (2012). Singapore schools and professional learning communities: Teacher professional development and school leadership in an Asian hierarchical system. Educational Review, 64(4), 405-424.

Hargreaves, A. P., \& Shirley, D. L. (Eds.). (2009). The Fourth Way: The Inspiring Future for Educational Change. Thousand Oaks, CA: Corwin Press.

Hargreaves, A., \& Fullan, M. (2015). Professional capital: Transformng teaching in every school. Teachers College Press.

Hairon, S., Goh,J.W. P., \& Chua, C. S. K. (2015).Teacher leadership enactment in professional learning community contexts:Towards a better understanding of the phenomenon. School Leadership \& Management, 35(2), 163-182.

Hairon, S., \& Tan, C. (2017). Professional learning communities in Singapore and Shanghai: Implications for teacher collaboration. Compare: A Journal of Comparative and International Education, 47(1), 91-104.

Heng, S. K. (2012a). Keynote Address by Mr Heng Swee Keat, Minister for Education, at the Ministry of Education Work Plan Seminar 2013, on Wednesday, 12 September 2012 at 9.20 a.m. at Ngee Ann Polytechnic Convention Centre. Retrieved from https:// www.moe.gov.sg/news/speeches/keynote-address-by-mr-heng-swee-keat-ministerfor-education-at-the-ministry-of-education-work-plan-seminar-on-wednesday-12september-2012-at-920-am-at-ngee-ann-polytechnic-convention-centre.

Heng, S. K. (2012b). Speech by Mr Heng Swee Keat, Minister for Education at the 15th Appointment and Appreciation Ceremony for Principals on Thursday, 27 December 2012, at Island Ballroom, Shangri-La Hotel. Retrieved from https://www.moe.gov. $\mathrm{sg} /$ news/speeches/speech-by-mr-heng-swee-keat-minister-for-education-at-the15th-appointment-and-appreciation-ceremony-for-principals-on-thursday-27december-2012-at-island-ballroom-shangri-la-hotel\#sthash.siqNTkri.dpuf.

Heng, S. K. (2013). Keynote Address by Mr Heng Swee Keat, Minister for Education, at the Ministry of Education Work Plan Seminar 2013, on Wednesday, 25 September 2013 at 9.15 a.m. at Ngee Ann Polytechnic Convention Centre. Retrieved from https://www.moe. gov.sg/news/speeches/keynote-address-by-mr-heng-swee-keat-minister-for-educationat-the-ministry-of-education-work-plan-seminar-2013-on-wednesday-25-september2013-at-9:15am-at-ngee-ann-polytechnic-convention-centre\#sthash.64vu3zhI.dpuf.

Heng, S. K. (2014a). Keynote Address by Mr Heng Swee Keat, Minister for Education, at the Ministry of Education Work Plan Seminar 2014, on Tuesday, 23 September 2014 at 9:15 a.m. at Ngee Ann Polytechnic Convention Centre. Retrieved from https:// www.moe.gov.sg/news/speeches/keynote-address-by-mr-heng-swee-keat-ministerfor-education-at-the-ministry-of-education-work-plan-seminar-2014-on-tuesday-23september-2014-at-9-15am-at-ngee-ann-polytechnic-convention-centre.

Heng, S. K. (2014b). Speech by Mr Heng Swee Keat, Minister for Education at the 17th Appointment and Appreciation Ceremony for Principals, Shangri-La Hotel, 30 December 2014, at 3.10 p.m. Retrieved from https://www.moe.gov.sg/news/speeches/ speech-by-mr-heng-swee-keat-minister-for-education-at-the-17th-appointmentand-appreciation-ceremony-for-principals-shangri-la-hotel-30-december-2014at-310pm\#sthash.tGGee3ry.dpuf.

Ho, P. (2014). Opening Remarks by Ms Ho Peng, Director-General of Education, at The Principals' Forum and PISA 2012 Problem Solving Results Launch on 31 March 2014, 2.30 p.m. at Victoria Junior College. Retrieved from https://www.moe.gov.sg/news/ 
speeches/opening-remarks-by-ms-ho-peng-director-general-of-education-at-theprincipalsand8217-forum-and-pisa-2012-problem-solving-results-launch-on-31march-2014-230pm-at-victoria-junior-college\#sthash.AW1niJRU.dpuf.

Ho, J.-M., \& Koh, T.-S. (2018). Historical Development of Educational Leadership in Singapore (pp. 29-83). In:T. S. Koh, \& D. Hung (Eds.), Leadership for Change: The Singapore Schools' Experience. Singapore: World Scientific.

Ho, J., Ong, M., \& Tan, L. S. (2019). Leadership of professional learning communities in Singapore schools: The tight-loose balance. Educational Management Administration \& Leadership, 1741143219833698.

Hogan, D. (2014). Why Is Singapore's School System So Successful, and Is It a Model for the West?. Retrieved from http://theconversation.com/why-is-singapores-school-systemso-successful-and-is-it-a-model-for-the-west-22917.

Hogan, D., \& Gopinathan, S. (2008). Knowledge management, sustainable innovation, and pre-service teacher education in Singapore. Teachers and Teaching: Theory and Practice, 14(4), 369-384.

Jackson, D., \& Temperley, J. (2007). From Professional Learning Community to Networked Learning Community (pp. 45-62). In: L. Stoll, \& K. S. Louis (Eds.), Professional Learning Communities: Divergence, Depth and Dilemmas. Berkshire: Open University Press.

Lave, J., \& Wenger, E. (1991). Situated learning: Legitimate peripheral participation. Cambridge university press.

Lee, D., \& Lee, W. O. (2013). A professional learning community for the new teacher professionalism: The case of a state-led initiative in Singapore schools. British Journal of Educational Studies, 61(4), 435-451.

Lim-Chan, G. C. Y. (2006). The Role of Heads of Department in Cluster Secondary Schools in Singapore. Doctor of Education, University of Leicester.

Ministry of Education. (2012, September 12). MOE Removes Secondary School Banding and Revamps School Awards [Press Release]. Retrieved from Ministry of Education website: https://www.moe.gov.sg/news/press-releases/moe-removes-secondary-schoolbanding-and-revamps-school-awards.

Ng, P.T. (2003). The Singapore school and the School Excellence Model. Educational Research for Policy and Practice, 2(1), 27-39.

$\mathrm{Ng}$, P. T. (2007). Quality assurance in the Singapore education system in an era of diversity and innovation. Educational Research for Policy and Practice, 6(3), 235-247.

$\mathrm{Ng}$, P.T. (2008). The phases and paradoxes of educational quality assurance: The case of the Singapore education system. Quality Assurance in Education, 16(2), 112-125.

$\mathrm{Ng}$, P. T. (2013). An examination of school accountability from the perspective of Singapore school leaders. Educational Research for Policy and Practice, 12(2), 121-131.

OECD. (2016). PISA 2015 Results in Focus. Retrieved from https://www.oecd.org/pisa/ pisa-2015-results-in-focus.pdf.

Porritt, V., Spence-Thomas, K., \& Taylor, C. (2017). Leading Professional Development (pp. 121-130). In: P. Earley, \& T. Greany (Eds.), School Leadership and Education System Reform. London: Bloomsbury.

Seah, J. C., \& Ow, A. (2003). School Excellence Model. From https://www.cscollege.gov.sg/ Knowledge/Ethos/Ethos\%20June\%202003/Pages/The\%20School\%20Excellence\%20 Model.aspx (Retrieved 26 April 2017).

Shanmugaratnam, T. (2005). Speech by Mr Tharman Shanmugaratnam, Minister for Education, at the MOE Workplan Seminar 2005, 22 September 2005 at 10.00 a.m. at the Ngee Ann Polytechnic Convention Centre. Retrieved from http://www.nas.gov.sg/ archivesonline/speeches $/$ view-html?filename=20050922991.htm. 
Teo, C. H. (1997). Improving School Management through School Clusters: Speech by Minister for Education, RADM (NS) Teo Chee Hean at the Official Opening of the New Campus of Tanjong Katong Girls' School on Friday 11 July 1997. Retrieved from https://www.moe.gov.sg/media/speeches/1997/110797.htm.

Teo, C. H. (1998). Principals - The Key Leaders in Building Thinking Schools Based on Strong School-Home-Community Relations: Speech by RADM Teo Chee Hean, Minister for Education and Second Minister for Defence at the Appointment Ceremony for Principals on Monday 21 December 98 at the Marina Mandarin Ballroom at 3 p.m. Retrieved from https://www.moe.gov.sg/media/speeches/1998/261298.ht.

Teo, C. H. (1999). Speech by RADM Teo Chee Hean, Minister for Education and Second Minister for Defence at the MOE Work Plan Seminar on Saturday 4 September 1999 at the Temasek Polytechnic at 9.25 a.m. Retrieved from http://www.nas.gov.sg/ archivesonline/data/pdfdoc/1999090403/tch19990904d.pdf.

Teo, C. H. (2000a).Ability Driven Education-Putting the System in Place: Speech by RADM Teo Chee Hean, Minister for Education and Second Minister for Defence at the MOE Work Plan Seminar 2000 on Saturday 23 September 2000 at 9.20 a.m. at the Nanyang Polytechnic Auditorium. Retrieved from https://www.nas.gov.sg/archivesonline/data/ pdfdoc/2000092302.htm.

Teo, C. H. (2000b). Dynamic School Leaders and Schools-Making the Best Use ofAutonomy: Speech by RADM (NS) Teo Chee Hean, Minister for Education and Second Minister for Defence at the Diploma ins Educational Administration (DEA) Graduation Dinner on Wednesday 12 January 2000, at 8 p.m., at the Belvedere, Mandarin Hotel, Singapore. Retrieved from http://www.nas.gov.sg/archivesonline/data/pdfdoc/2000011201/ tch20000112b.pdf.

Teo, C. H. (2002). Speech by RADM Teo Chee Hean, Minister for Education and Second Minister for Defence at the Appointment Ceremony for Principals on Monday, 30 December 2002 at 3 p.m., Island Ballroom, Shangri-La Hotel. Retrieved from https:// www.moe.gov.sg/media/speeches/2002/sp30122002.htm.

The Straits Times. (2015). Heng Swee Keat as Education Minister: A Study in Bold Moves. Retrieved from http://www.straitstimes.com/singapore/education/heng-sweekeat-as-education-minister-a-study-in-bold-moves.

The Straits Times. (2019). China Pips Singapore to Top Sport in Pisa Education Ranking. The Straits Times. Retrieved from https://www.straitstimes.com/singapore/education/ china-pips-spore-to-top-spot-in-pisa-education-ranking.

The Ministry of Education, Singapore. (2019a). School Information Service-School Clusters and Cluster Superintendents. Retrieved from https://sis.moe.gov.sg/SchoolClusters.aspx.

The Ministry of Education, Singapore. (2019b). Schools. Retrieved from https://www.moe. gov.sg/about/org-structure/sd.

Wee, H. T., \& Chong, K. C. (1990). 25 years of school management. Evolution of Educational Excellence, 25, 31-58.

Wee, H.T., \& Chong, K. (1994). 25 Years of School Management (pp. 33-60). In: J. S. K.Yip, \& W. K. Sim (Eds.), Evolution of Educational Excellence: 25 Years of Education in the Republic of Singapore. Singapore: Longman Singapore Publishers Pte Ltd. 


\section{9}

\section{EDUCATIONAL TECHNOLOGY TO IMPROVE CAPACITY - INTEGRATING ADAPTIVE EDUCATION PROGRAMMES IN PUBLIC SCHOOL IN KENYA}

\section{Tom Kaye}

\section{Introduction}

Since the beginning of the new millennium two phenomena have increased global interest in, and international comparisons of, national education outcomes. The first phenomenon was the rise in prominence of international assessments. In 1995, the International Association for the Evaluation of Educational Achievement executed the first Trends in International Mathematics and Science Study (TIMSS). The study, which included 46 countries, was the largest international comparison of national education outcomes undertaken to the time. The Programme for International Student Assessment, introduced in 2000, measures reading, mathematics and science outcomes. These tools track national performance over time and allow international comparisons and benchmarking. The second phenomenon was the introduction of the Millennium Development Goals (MDGs). The second MDG (achieving universal primary education) placed a global spotlight on countries yet to achieve full primary enrolment. This was amplified in 2015 when the Sustainable Development Goals (SDGs) were introduced. The fourth SDG (ensuring inclusive and equitable quality education and promoting lifelong learning opportunities for all) broadened the focus from enrolment to quality. The increased ability to track and compare outcomes, coupled with greater global scrutiny, has increased pressure on policymakers to realise rapid improvements in learning. This pressure is particularly intense in countries where universal enrolment is not yet achieved or learning outcomes lag behind global averages.

In the cases of lower and middle-income countries (LMICs), the demand for rapid improvement in learning outcomes often cannot be met through existing low-performing systems which lack foundational inputs, including adequate infrastructure, robust curriculum or well-trained teachers. This has led countries 
to adopt system-wide reforms that attempt to holistically address multiple challenges and align education systems towards learning. However, these reforms are complex, politically sensitive, and often painstakingly slow (Crouch and Destefano, 2015). Kaffenberger and Spivack (2019) demonstrate that if the current pace of change in education outcomes in non-OECD countries continues, it will take over 100 years for these countries to reach the current OECD average.

This slow pace of change has made educational technology, or EdTech, increasingly appealing to policymakers trying to fast-track improvements in learning outcomes. EdTech comes in many shapes and sizes, with the term referring to tools that might deliver curriculum, support assessment, or streamline school management. As early as 2006, the Inter-American Development Bank was supporting more than 38 EdTech projects in 20 countries (Arias Ortiz and Cristia, 2014). Isaacs' (2015) study identifies more than 50 EdTech projects scheduled to take place in 20 African and Mediterranean countries between 2015 and 2021. However, the impact of EdTech has been varied. While some well-designed projects have led to improved service delivery or outcomes, there are countless examples where EdTech has been deployed as a silver bullet to resolve complex challenges, only for poorly designed programmes to waste valuable resources (Batty et al., 2019).

Although EdTech has the potential to support better learning outcomes, we have much to learn about successfully deploying these tools. This chapter provides a modest contribution by providing insight into how EdTech can be harnessed to drive better learning outcomes in LMICs. Kenya was selected as a case study due to its regional leadership in the EdTech space. Two initiatives form the centrepiece of EdTech integration into public education in Kenya the Digital Literacy Programme (DLP) and the Tusome (which stands for Let's Read in Kiswahili) programme. The two programmes adopted vastly different approaches to programme design and implementation, including how they generated trust amongst programme personnel, built capacity to implement the reforms and ensured that participants were accountable for using the EdTech tools. Through exploring the Kenyan case, it becomes clear that while EdTech can play a role in improving education service delivery, its impact in LMICs is the greatest when integrated into reforms designed to address specific challenges impeding the effectiveness of education provision. The chapter reveals how maximising the impact of these tools is contingent on deploying EdTech to support well-defined activities within robustly designed programmes, ensuring users have the capacity to leverage the tools to drive better education outcomes, and putting in place mechanisms to hold users accountability for deploying the technology in line with their intended use.

\section{The context of Kenya's primary education system}

Since the 1990s, spurred by entrenched, low, learning outcomes, Kenya has pursued two waves of education system reform. The first, from 2003 to 2013, expanded access to education. The second, from 2013 to the current day, 
aims to increase education quality. Through these reforms, Kenya is building a robust education system which acknowledges and addresses its complex socio-economic, political and cultural context. However, learning levels are still below international benchmarks, with innovation required to address the remaining challenges.

\section{The Kenyan development context}

Kenya is one of the world's largest, most well-populated and most ethnically diverse countries. The nation's education services traverse great distances and meet the needs of a large and diverse population. The country's median age of 19.7 years is half that of the United States and China and lower than many regional neighbours. In 2017, GDP per capita was US\$1594, making it the 153rd poorest of the world's 195 countries (World Bank, 2019a,b). Other macro-level challenges impeding access to and completion of education include economic inequality, poor governance, corruption and weak infrastructure. Table 9.1 provides greater insight into Kenya's complex macro socio-economic context.

At the micro level, various challenges limit children accessing education. Primarily, child labour persists. This is despite Kenya having ratified international conventions and put in place national legislation to protect children. Nearly $66 \%$ of children aged 5-11 work at least 1 hour of paid work a week, and more than 10\% aged 12-14 work more than 14 hours a week (Kenya National Bureau of Statistics and United Nations Children's Fund, 2011). Work includes supporting family businesses, agricultural activities, domestic service and begging. Additional micro-level challenges include long distances to travel to schools, inadequate food, teenage pregnancy and threats to personal security.

TABLE 9.1 Key national statistics impacting educational context in Kenya

\begin{tabular}{llll}
\hline Indicator Name & Indicator Result & Indicator Name & Indicator Result \\
\hline Total population & 52.2 million (2019) & Access to electricity & $56 \%$ \\
$\begin{array}{l}\text { Population growth } \\
\text { rate }\end{array}$ & $2.5 \%$ per annum & Infant mortality & $\begin{array}{c}52 \text { of every } 1000 \\
\text { children born }\end{array}$ \\
$\begin{array}{l}\text { Proportion of } \\
\text { population in poverty }\end{array}$ & $\begin{array}{l}\text { US } \$ 1.90 \text { per day) } \\
\text { Life expectancy }\end{array}$ & Net migration & $\begin{array}{c}-50,000 \text { people per } \\
\text { annum }\end{array}$ \\
Fertility rate & 64 Male/69 Female & Mobile phone access & $86.1 \%$
\end{tabular}

Source: Adapted from United Nations Population Fund. (2019). World Population Dashboard: Kenya. From https://www.unfpa.org/data/world-population/KE (Retrieved 22 May 2019) (United Nations Population Fund, 2019); World Bank. (2019a). Net Official Development Assistance Received (Current US\$). Kenya: Data. From World Bank Open Data website https://data.worldbank.org/indicator/DT.ODA.ODAT. CD?locations=KE (Retrieved 28 October 2019); and World Bank. (2019b). World Development Indicators Database. From World Bank Open Data website https://data.worldbank.org/country/kenya?view=chart (Retrieved 20 February 2019). 
In understanding Kenya's development context, it is important to acknowledge the role of development partners. In 2017 , Kenya received nearly US $\$ 2.48$ billion aid from domestic and international sources, including UN agencies, development banks and international and local NGOs (World Bank, 2019a). These agencies contribute funding and technical advice to support infrastructure, institutional and social development. However, these contributions also bring complexities. For instance, engaging with so many diverse partners can fragment national development priorities. Partners have strong views about how to address challenges, leading governments to adopt programmes from other contexts rather than homegrown approaches aligned to local need (Colclough and Webb, 2010). Finally, variations in funding can impede absorption and development effectiveness. In recent years, aid in Kenya has ranged from US $\$ 388$ million (2002) to over US $\$ 3.3$ billion (2013). Ensuring institutional structures are in place to implement programmes can take time. Variations in financing make engaging resources difficult, meaning funding does not always generate the expected outcomes.

\section{5-2013: Persistently low learning outcomes lead to reform}

While Kenya's regional assessment results between 1995 and 2011 compared favourably with its neighbours, outcomes were stagnant and lagged behind other international comparators. The Southern and Eastern Africa Consortium for Monitoring Educational Quality (SACMEQ ${ }^{1}$ ) assessment results highlight that despite performing above the regional average in reading and mathematics in SACMEQs I-III, Kenya's results regressed over time (see Table 9.2). Using Altinok et al.'s (2018) method to compare various regional and international

TABLE 9.2 Kenyan SACMEQ results over time

\begin{tabular}{lcccc}
\hline & $\begin{array}{c}\text { SACMEQ I } \\
(1995-2000)\end{array}$ & $\begin{array}{c}\text { SACMEQ II } \\
(2000-2005)\end{array}$ & $\begin{array}{c}\text { SACMEQ III } \\
(2007-2011)\end{array}$ & Change Over Time $^{a}$ \\
\hline $\begin{array}{l}\text { Kenya Reading } \\
\text { Mean Score }\end{array}$ & 543.3 & 546.5 & 543.1 & -0.2 \\
$\begin{array}{c}\text { Kenya Math } \\
\text { Mean Score }\end{array}$ & 563.3 & 557 & -6.3 \\
Regional average & 500 & 500 & 500 & N/A \\
\hline
\end{tabular}

${ }^{a}$ Change over time of Reading Mean Score is measured between SACMEQ I and SACMEQ III. For Math Mean Score, the change is measured from the first execution in SACMEQ II and the second in SACMEQ III.

Source: Adapted from Onsomu, E., Nzomo, J., \& Obiero, C. (2005). The SACMEQ II Project in Kenya: A Study of the Conditions of Schooling and the Quality of Education. In: SACMEQ Educational Policy Research Series. Retrieved from http://www.tzdpg.or.tz/fileadmin/documents/dpg_internal/dpg_working groups_clusters/cluster_2/education/3-Core_Documents/SACMEQ_Tanzania_2005.doc (Onsomu et al., 2005) and Ogle, M. A., Wasanga, P. M., \& Wambua, R. M. (2012). The SACMEQ III Project in Kenya: A Study of the Conditions of Schooling and the Quality of Education. Retrieved from www.knec.ac.ke (Ogle et al., 2012). 
tests, Kenya's SACMEQs I-III results convert to a score of approximately 425, slightly above the 'low' threshold of 400, and significantly below the 'intermediate' achievement threshold of 475. Additionally, findings from the UWEZO project identified that in annual tests conducted from 2011 to 2014, a maximum $41 \%$ of Kenyan children up to age 13 could finish basic literacy and numeracy tasks expected of a 7-year-old child (Uwezo, 2015).

In order to address these low learning outcomes, Kenya's education leadership began various reforms aimed at progressively developing a stronger education system.

While not aimed at increasing education quality, the first major reform was the introduction of universal primary education. Ever since independence in 1963 Kenya aspired to universalise education. In 2002, with the results of SACMEQ I providing a catalyst, free primary education for all was announced. The policy addressed a major barrier to enrolment - cost. Schools are now allocated US $\$ 10$ per pupil to cover expenses. Parents, who had covered $35 \%$ of costs, no longer paid any fees (Gichura, 2003). Net enrolment increased from 59.5\% in 2002 to $76 \%$ in 2009, while attendance also increased (World Bank, 2019b). Although the policy increased access, it negatively impacted education quality, with teachers, materials and infrastructure now split between more students (Bold et al., 2010). This decrease in quality likely contributed to the fall in outcomes between SACMEQs II and III.

The second major reform was the introduction of a new Basic Education Act in 2010. The new national constitution, also introduced in 2010, intended to make democracy and governance more representative and inclusive, devolving responsibilities to officials in 47 counties and 290 sub-counties. In education, this meant creating county and sub-county offices in each municipality to allow the Ministry of Education (MOE) to better meet local need. This changed how schools interact with the MOE, with county officers becoming responsible for quality assurance, inspection and teacher training. While county personnel have some autonomy to determine how to best support schools, hierarchical control is maintained through budget allocation and rigid inspection schemes.

The third major reform was an increase in the independence of the Teachers Service Commission (TSC). The TSC trains, employs, promotes and disciplines teachers. While the TSC's mandate and operations did not change, their increased independence reduced the MOE's involvement in these activities. Where previously the TSC was required to comply with requests from MOE, this is no longer the case. The TSC can now unilaterally manage the teaching workforce and change policies or processes. This independence has led to some challenges implementing projects, collecting governance and accountability data and, generally, ensuring activities align with MOE policy. However, despite this formal separation, informal collaboration often occurs between MOE and TSC officers to support schools. While this informal collaboration is positive, the lack of a formal foundation means the MOE is not able to provide full oversight of the sector. 


\section{Onwards: a shift to focus on quality}

Since the start of reforms in 2003, Kenyan assessment results have improved (Table 9.3). This goes beyond a rebound from the dip seen in SACMEQ III, with an increase in both reading (6\%) and math (nearly 10\%) scores between SACMEQs III and IV (Ministry of Education, 2018). Using the harmonised test scores, this equates to approximately 455 on the TIMSS scale, moving Kenya closer to the intermediate benchmark of 475 .

However, while results have improved, Kenya still lags behind global averages. Analysis of the determinants of learning identified major barriers to learning, including the quality of both inputs (e.g. curriculum, textbooks, materials and teacher capacity) and oversight systems (e.g. teacher and school monitoring) (Ngugi et al., 2015). Noting the findings of these diagnostics, the MOE has introduced various reforms aimed at increasing Kenyan quality education.

The most important quality-focused initiative is Kenya's curriculum reform. In line with international good practice, Kenya is adopting a competency-based curriculum incorporating critical thinking and active learning. Curriculum reform, and the pedagogical changes accompanying it, is controversial and difficult to execute. Acknowledging this, Kenya made robust preparations and is diligently pursuing the reform. However, despite careful planning, communication and teacher training, in March 2019, the Secretary General of the Kenyan National Union of Teachers demanded the MOE suspend the new reform, arguing teachers did not have the capacity to implement the new curriculum. Prof. George Magoha, Cabinet Secretary for Education, resisted, vowing to deliver the reform (Wanzala, 2019).

In parallel to the curriculum reform, the TSC is implementing a new Teacher Performance Appraisal and Development (TPAD) tool to strengthen teacher management and capacity development practices. The TPAD replaces the previous system, a black box lacking a focus on skills growth or career progression, with a more transparent process that places teacher development at the centre. Teachers self-appraise their skills and performance, while their supervisor assesses them on the same metrics. The two discuss the outcomes and collaboratively finalise the appraisal. This increases the teacher's awareness of their strengths and helps them identify mechanisms to address weaknesses. It also fosters a more

TABLE 9.3 Kenyan SACMEQs III and IV results

\begin{tabular}{lccc}
\hline & $\begin{array}{c}\text { SACMEQ II } \\
(2000-2005)\end{array}$ & $\begin{array}{c}\text { SACMEQ III } \\
(2007-2011)\end{array}$ & $\begin{array}{c}\text { SACMEQ IV } \\
(2013-2017)\end{array}$ \\
\hline Reading Mean Score & 546.5 & 543.1 & 577.6 \\
Math Mean Score & 563.3 & 557.0 & 608.1 \\
\hline
\end{tabular}

Source: Adapted from Ministry of Education. (2018). 2018 Education Quality Dialogues. Retrieved from http://www.education.go.ke/images/GPE_PRIEDE/2018-FINAL-DIALOGUES-.pdf. 
trusting relationship between teachers and supervisors. The TPAD underpins efforts by the TSC to increase the professionalism of the teacher workforce, which is expected to result in better education outcomes.

The MOE is also working to increase community engagement in schools. The school board of management (BOM), a localised governing body comprised parents and other community members, oversees operations at the school level. The BOM supports the development of both physical infrastructure and high-quality teaching approaches, liaises with the county office on issues, such as staffing and discipline, and oversees child welfare and the achievement of learning outcomes. This increased community involvement leverages social accountability approaches, ensuring MOE and TSC oversight is complemented by grassroots level accountability driven by parents and local stakeholders.

While these quality-focused reforms are still in the early stages, initial indications are they are having a positive impact. An analysis of 40 schools nationally revealed that academic results have improved, BOMs are constituted and are running effectively and schools are more child friendly. While these results are only early and limited, they demonstrate that Kenya is moving in the right direction.

\section{Enhancing education quality through the use of EdTech in Kenya}

While Kenya has managed to realise modest improvements in learning outcomes, this suite of reforms has taken approximately 15 years to begin to deliver results. During this same period, a young population with a thirst for education has emerged and is impatient for better education. The MOE is reluctant to wait the many years required for a full system overhaul using traditional approaches.

In an attempt to fast-track improved outcomes, the MOE reviewed the latest trends in international education delivery and identified the option of using EdTech to enhance the efficiency and effectiveness of education service delivery. This section provides an overview of the evolution of the global EdTech industry before analysing Kenya's experience designing and implementing two large-scale EdTech initiatives in the public education system.

\section{A brief history of EdTech}

Initially, technological innovation in the education sector was slow and focused on infrastructure and communication. However, innovation is now accelerating, with investments in EdTech booming. In 2017, investments rose to US $\$ 9.52$ billion, an increase of nearly 600\% from 2013 (Singh, 2018). EdTech innovations are increasing efficiency and effectiveness in areas, including access and provision, teacher training, content delivery, student assessment and system management.

However, while EdTech has great potential, the industry is still immature, and robust evidence of successful practices is lacking. Due to the fragmented way in which the industry emerged globally, few resources document the evolution 
or current state of EdTech (Weller, 2018). This lack of history and global perspective makes it difficult for professionals to understand what does or does not work, or build on an existing body of knowledge. Additionally, there is a lack of a formal language to discuss and analyse the industry. Finally, and most importantly, despite the growing prevalence of EdTech projects, there is little evidence to demonstrate that EdTech is significantly impacting learning outcomes (Escueta et al., 2017).

Despite the immaturity of the industry and the risks involved in the development and roll-out of EdTech, the slow change using traditional methods has encouraged education leaders in LMICs more broadly, and Kenya specifically, to explore EdTech to fast-track improvements in learning outcomes. Kenya was one of the first developing countries to have implemented multiple large-scale EdTech programmes, providing the opportunity to explore lessons learned across two programmes rolled out in a single context.

\section{EdTech in Kenya: the Digital Literacy Programme}

The DLP was announced in 2013 by the Coalition of the National Alliance, the United Republican Party, the National Rainbow Coalition and the Republican Congress Party. These parties aimed to equip all Kenyan schoolchildren with a laptop computer. This ambition went through various iterations, with the scope narrowing over time. The second medium-term plan 2013-2017 earmarked US\$270 million to distribute laptops to schools, enhance infrastructure, train teachers and develop online content. The National Education Sector Plan 2013/14-2017/18 stated that by the end of 2018, all grade 1 pupils would have a laptop and one computer would be accessible between 15 students in other classes. Finally, in May 2016, the government advised that the DLP would provide all grade 1 students with tablets, with teachers receiving laptops (Wanzala and Nyamai, 2018).

Throughout its evolution from promise to policy to plan, the DLP was met with both excitement and scepticism. Advocates pointed to the DLP's potential to enhance Kenya's education equity and quality in the short term and its global competitiveness in the long term. Critics questioned whether the government would be able to deliver the programme in a timely and cost-effective manner, and whether the planned activities would lead to the stated outcomes (Barmao, 2014). Potential challenges included ensuring adequate financing for devices and capacity development, delivering teachers adequate training in the new tools, putting in place secure storage and ensuring alignment between the DLP and existing reform programmes. Stakeholders also argued the US $\$ 270$ million could be better used to support existing infrastructure development, curriculum and pedagogical reform programmes (Odero, 2016).

Despite the opposition, and the narrower than originally anticipated scope, once implementation began in 2016 the roll-out accelerated quickly. Major achievements within 6 months included training approximately 70,000 teachers by mid-2016, commencing device delivery in September 2016, creating digital 
content for grades 1 and 2 in Kiswahili, English, mathematics, science and social studies and connecting all but 500 schools to electricity (Mariga et al., 2017).

Despite initial progress, the fears of critics were realised, with various challenges limiting the DLP's impact. The goal of reaching all schools by December 2016 was not achieved. By July 2018, only 19,000 of the 23,000 schools had received devices (Wanzala and Nyamai, 2018). Teacher training was also inadequate. Only 70,000 teachers (out of approximately 250,000) received training by the end of 2017. The lack of capacity development compounded teachers' negative perceptions of the programme, which in turn led to limited uptake (Nyaga, 2018). In 2018, only just over a third of teachers reported they were using the tablets in the classroom despite having received the devices (Wanzala, 2018).

In February 2019, the MOE's Principal Secretary announced that the ministry had suspended the DLP. Evidence indicated that although supply and installation was at nearly 95\%, utilisation had only reached approximately $5 \%$.

\section{EdTech in Kenya: Tusome}

Tusome, which translates to 'Let's Read' in Kiswahili, is a reform programme focused on enhancing Kenyan early grade literacy (EGL) quality. It aims to strengthen EGL practices in grades 1, 2 and 3 in all 23,000 Kenyan public schools by (1) providing new textbooks and materials, (2) training all grades 1-3 teachers in the new EGL approaches, (3) training Civil Society Organisation (CSOs) in the new approaches to help them support teachers and (4) training senior leaders to use Tusome data to identify and address education system gaps (U.S. Embassy in Kenya, 2016). EdTech is used in Tusome to support teacher coaching and oversight. All CSOs receive tablets that make classroom observations more efficient and effective while also supporting more robust data collection. The tablets are equipped with five resources:

1. Tangerine ${ }^{\circledR}$ : Tutor is a classroom observation tool created for Tusome. CSOs record observations on the tablet during the lesson. They then assess the performance of three students using tests on the tablet. Results are synthesised to identify ways to improve teacher pedagogy.

2. A digital version of all Tusome textbooks and materials for easy access during school visits.

3. 30 Videos demonstrating how to effectively teach using the Tusome instructional approach.

4. Papaya ${ }^{\mathrm{TM}}$ helps teachers identify and pronounce letters in Kiswahili and English.

5. A dashboard of data is accessible by CSOs, County Directors of Education and central MOE staff. The data displays national EGL performance and supports evidence-based policymaking.

The inclusion of EdTech in Tusome built on the robust foundations laid by the Primary Maths and Reading (PRIMR) programme (RTI International, 
2014). Running from 2011 to 2014, PRIMR included randomised control trials piloting various EdTech interventions.

The Tusome midline evaluation report found statistically significant increases in EGL in both Kiswahili and English (Freudenberger and Davis, 2017, p. 7). Other studies reveal the positive impact the tablets have had on CSO and teacher behaviour (e.g. more than $98 \%$ of CSOs either agree or strongly agree that tablets facilitate more engaging feedback sessions and more than $90 \%$ either agree or strongly agree the tablets enable them to visit more teachers), which is expected to have been the key driver behind the increase in learning outcomes (Piper et al., 2017, 2018).

\section{Contrasting the Digital Literacy Programme and Tusome}

The DLP and Tusome have had vastly differing planning and implementation approaches and levels of success. Table 9.4 summarises key elements of the two programmes contributing to these differences.

\section{The role of trust, capacity and accountability in adopting EdTech in Kenya}

This section analyses how trust, capacity building and accountability affected the DLP and Tusome programmes. It highlights how the existence or absence of these variables can significantly impact EdTech deployment in both positive and negative ways. Importantly, the analysis also reveals how EdTech can help to strengthen trust, capacity and accountability within education systems. The findings of this analysis reinforce that EdTech as a stand-alone tool is not the silver bullet that many education policymakers seek. Rather, EdTech is at its most powerful when it is leveraged to make well-designed programmes focused on enhancing education quality more efficient and effective.

\section{The impact of Kenya's education reforms on trust, capacity and accountability}

When considering how the DLP and Tusome were impacted by, and impacted, trust, capacity and accountability in the Kenyan education system, it is important to recognise that the programmes were not deployed in a vacuum. Rather, they were introduced in a context that had recently been subject to various, significant, reforms. These reforms disrupted the levels of capacity and trust within the system and changed the nature and dynamics of the accountability mechanisms.

The reforms prior to 2013 resulted in major shifts in the roles and responsibilities of, and relationships between, institutions and actors. For example, free primary education was not accompanied by an appropriate increase in teacher recruitment. This resulted in the student to teacher ratio increasing significantly. Teachers felt abandoned, resulting in an erosion of trust between teachers and the 
TABLE 9.4 Contrast of key traits of DLP and Tusome interventions

\begin{tabular}{|c|c|c|}
\hline & Digital Literacy Programme & Tusome \\
\hline $\begin{array}{l}\text { Origin and } \\
\text { design }\end{array}$ & $\begin{array}{l}\text { - Forged from a political } \\
\text { promise }\end{array}$ & $\begin{array}{l}\text { - Based on robust evaluation of } \\
\text { previous pilot }\end{array}$ \\
\hline Purpose & $\begin{array}{l}\text { - Broad purpose, aimed to } \\
\text { increase digital literacy and } \\
\text { general learning } \\
\text { - Scope regularly changed } \\
\text { over time, including during } \\
\text { implementation }\end{array}$ & $\begin{array}{l}\text { - Well defined purpose. Used tech } \\
\text { to make coaching more efficient } \\
\text { (more time with teachers) and } \\
\text { effective (more engaging) } \\
\text { - Aligned with the theory that } \\
\text { enhanced teacher quality leads } \\
\text { to better outcomes }\end{array}$ \\
\hline Implementation & $\begin{array}{l}\text { Encountered regular delays } \\
\text { due to budgetary constraints, } \\
\text { infrastructure limitations and } \\
\text { lack of uptake }\end{array}$ & $\begin{array}{l}\text { - Complemented new EGL } \\
\text { approaches by enhancing } \\
\text { coaching process } \\
\text { - Detailed 5-year plan } \\
\text { implemented in line with } \\
\text { expectations }\end{array}$ \\
\hline $\begin{array}{l}\text { Training and } \\
\text { support }\end{array}$ & $\begin{array}{l}\text { - Less than } 30 \% \text { of } 250,000 \\
\text { teachers trained, with devices } \\
\text { not arriving until last day } \\
\text { - Little evidence of ongoing } \\
\text { support or implementation } \\
\text { policies/manuals }\end{array}$ & $\begin{array}{l}\text { - Training focused on pedagogy. } \\
\text { Tablets introduced after } \\
\text { technical training } \\
\text { - Teachers and CSOs both } \\
\text { trained to ensure alignment } \\
\text { - Guides provided to support } \\
\text { implementation }\end{array}$ \\
\hline $\begin{array}{l}\text { Stakeholder } \\
\text { engagement }\end{array}$ & $\begin{array}{l}\text { Ambivalent public feedback } \\
\text { did not impact pursuit of } \\
\text { political promise } \\
\text { - Lack of formal communication } \\
\text { led to weak stakeholder } \\
\text { engagement }\end{array}$ & $\begin{array}{l}\text { - Communication to stakeholders } \\
\text { and from conception through } \\
\text { implementation } \\
\text { - Included midline evaluation, } \\
\text { with results publicly available } \\
\text { to demonstrate impact }\end{array}$ \\
\hline Oversight & $\begin{array}{l}\text { Little formal oversight or } \\
\text { accountability to ensure } \\
\text { teachers used devices }\end{array}$ & $\begin{array}{l}\text { Tablets equipped with inbuilt } \\
\text { oversight mechanisms. GPS } \\
\text { locking meant data could only } \\
\text { be entered while on school } \\
\text { grounds } \\
\text { - Automated data upload allowed } \\
\text { county and central personnel to } \\
\text { oversee CSO progress }\end{array}$ \\
\hline Impact & $\begin{array}{l}\text { - No impact on learning } \\
\text { - Programme cancelled in } \\
\text { February } 2019\end{array}$ & $\begin{array}{l}\text { Midline evaluation indicated } \\
\text { statistically significant impact } \\
\text { on learning outcomes } \\
\text { - Assessments show CSOs are } \\
\text { using tablets and they are helping } \\
\text { them in their work }\end{array}$ \\
\hline
\end{tabular}


MOE (UNESCO, 2005). Decentralising activities to the county level required a new workforce to be recruited and trained, and new relationships to be developed. The newer, more independent, TSC created a split in the accountability system. Rather than reporting to the MOE, teachers and school leaders were now solely accountable to the TSC, even though county-based MOE staff still conducted school inspections. Informal practices emerged to traverse institutional silos. These practices required trust between the CSOs, MOE staff and teachers, which had begun to slowly emerge.

The quality-focused reforms commencing from 2013 compounded the impact of the earlier reforms. The shift to the competency-based curriculum meant teachers who had used a rote-based approach for many years needed to learn new pedagogical approaches while simultaneously teaching more children. At the same time, the new teacher professional development tool shifted how teachers were assessed and held accountable, requiring the relationships between teachers and their supervisors to be redefined. Finally, teacher accountability was impacted by the increased role played by school BOM who had become more involved in teacher oversight and management.

While these reforms strengthened Kenya's education system, their impact on trust, capacity and accountability is significant. While a full exploration of these issues is beyond the scope of this chapter, recognising that these reforms impacted the system is important when analysing dynamics related to capacity, accountability and trust in relation to the DLP and Tusome programmes.

\section{Trust, capacity and accountability and the Digital Literacy Programme}

The DLP provides an excellent example of the importance of considering trust, capacity and accountability when designing and implementing EdTech interventions. Overlooking these elements played a large part in the eventual failure of the DLP.

While the design of the DLP attempted to leverage the high level of general technological trust in Kenya, the programme's design did not include adequate mechanisms to build trust in the DLP specifically. Kenya is home to many innovative tech-based tools which have become integrated into everyday life, driving a high level of general technological trust. For example, nearly a quarter of Kenya's gross national product is now transferred through the mobile banking application, MPESA, rendering cash and cards nearly obsolete (Mengitsu and Imende, 2013). In addition to this general trust, Mwingirwa et al.'s (2015) study found that teachers are welcoming of well-designed EdTech solutions that facilitate learning. As discussed earlier, despite this conducive environment, the DLP faced opposition from a wide section of the education community. Rather than working with stakeholders to address concerns, the government pursued the DLP, hoping to convert critics with successful implementation. While ignoring these concerns partially fractured stakeholder trust, this was compounded when 
implementation faced challenges. Instead of successful implementation building trust amongst the programme's opposition, the poor implementation experience crystallised and increased the existing distrust amongst stakeholders.

Stakeholder distrust of the DLP was compounded when capacity-building activities proved inadequate to realise the programme's goals. The attempts to build teacher capacity were inadequate in two significant ways. First, not enough teachers were trained. Initial training reached less than a third of Kenya's 242,000 teachers (Ministry of Education Science and Technology, 2014). Only three teachers per school received formal training, with additional teachers trained by those attending the first training. There was no mechanism to assess the knowledge or skills of those who received the initial training or the quality of the training they subsequently delivered to others within the school. As of May 2019, only an additional 10,980 teachers had received formal training (Kenyan ICT Authority, n.d.). Second, the training provided to teachers who did attend was inadequate. Three days was insufficient to equip teachers to integrate the tools into teaching practices. The training mainly covered device operations, with little focus on how teachers could use devices to improve learning. Limited training was compounded by a lack of publicly available documentation to support teachers to integrate the devices into their daily activities. The inadequate training programme further negatively impacted trust between programme stakeholders and those driving the reforms.

With teachers already unwilling and unable to use the devices, the DLP's challenges were accelerated by the absence of an accountability mechanism. Oversight of education in Kenya is split between the MOE and the TSC. At the county level, officers employed by the MOE are responsible for inspecting schools and providing guidance on strengthening practices and processes. Meanwhile, officers engaged by the TSC are responsible for providing guidance to teachers to improve pedagogical practices. For the DLP, there is an additional third actor - the ICT Authority - who is responsible for providing the devices, capacity development, broadband connectivity and content. None of these agencies were mandated to monitor and enforce device use. Additionally, existing accountability structures (e.g. the Guidelines for Quality Assurance and Standards Assessment of Schools in Kenya) have not been updated to reflect the DLP.

While the lack of trust, inadequate capacity development and the absence of accountability mechanisms limited the DLP's impact, this was compounded by the lack of consideration given to the transitory state of Kenya's education system. At a time when the sector was transitioning to new institutional structures and technical approaches, the money spent on the DLP (US $\$ 270$ million, close to the entire primary education budget for 1 year) could have been better used elsewhere. The decision to implement the DLP when the system was undergoing significant change placed pressure on teachers already struggling to understand new pedagogical approaches and resulted in reform fatigue amongst school, county and central staff already stretched supporting existing reform. This lack of consideration of the current state of the education sector reinforced initial stakeholder distrust of the DLP. 


\section{Trust, capacity and accountability and the Tusome programme}

In contrast to the DLP, Tusome's strategically targeted focus ensured designers and implementers could better address issues related to trust, capacity and accountability, which contributed to the programme's success.

Building capacity was at the heart of Tusome's design. The programme focused on training teachers and CSOs to implement, and provide coaching on, new EGL pedagogical approaches. Both teachers and CSOs attended a 2-day training to help them understand the new approaches, ensuring both sets of actors shared an understanding of the pedagogical approaches and the coaching role the CSOs would play. Ensuring teachers understood the CSO's role as coaches meant they were prepared to work collaboratively with CSOs when they visited schools. This was an important step as many of the new CSOs had formerly been employed as inspectors. Helping both CSOs and teachers understand the shift from inspecting and judging schools to supporting teachers to enhance teaching quality was an important step in building trust between the groups. This also created a feeling of collaborative accountability for children's learning outcomes. In addition to the foundational training, the CSOs received 1 day of training focused on how the tablets could support their coaching. The training emphasised that the tablets were tools to complement, rather than substitute for, the CSO's technical expertise. This strategy positioned the CSOs as trusted professionals tasked with working with teachers to strengthen the education system rather than as implementers of an EdTech project.

This comprehensive training programme was also part of a multifaceted strategy to build stakeholder trust in Tusome and foster trust between the different actors implementing the programme (Piper et al., 2018). The first step towards fostering trust in the programme was aligning international good practice with local need. The link between enhancing teacher quality and improving learning outcomes is globally recognised. Tusome's focus on enhancing teacher quality also aligned with Kenya's needs, with education stakeholders agreeing that more and higher quality teachers were required to support free primary education. Bringing international good practices to Kenya to address this local need helped foster trust in the programme. The second step was to ensure robust programme communications. The Tusome communication approach included strategically sharing or making available various programme documents, training materials and teacher guides. This ensured stakeholders were aware of Tusome's purpose and processes, and that all parties understood their, and each other's, roles in the programme. Making the documents publicly available built further trust in the programme amongst stakeholders and, provided a foundation for transparency between programme implementers. It also fostered an environment of networked accountability amongst the various actors. Third, programme goals were made clear to implementers and stakeholders, with performance updates made publicly available. This meant stakeholders understood programme progress, which generated even greater trust in the reform. Each of these three elements, as well as the robust capacity development 
programme, played an important role in fostering both stakeholder trust in the programme and trust between programme implementers.

The use of tablets played a vital role in fostering trust amongst both implementers and stakeholders by making data more accessible and transparent. This, in turn, made implementing accountability measures simpler and more effective. The data was collected, and used, at multiple levels. When undertaking coaching visits, CSOs used the tablet's student assessment function to assess learning, providing CSOs with insight into students' progress. The outcomes combined with lesson observations to inform discussions with teachers about pedagogical practices. The CSOs also captured additional school-level data such as teacher and student attendance, infrastructure information (e.g. classroom quality, number of desks and chairs) and progress through the curriculum. The data submitted by CSOs was used to support analysis of trends in counties, sub-counties or at a national level. The data identified strengths and weaknesses which informed the design of training programmes and system-level policy dialogue.

Beyond supporting better pedagogical practices, one of Tusome's most important contributions to Kenya's education system was fostering a positive approach to accountability. Piper et al. (2018) note, 'the Tusome feedback data were utilized to encourage greater levels of instructional support', rather than underpinning punitive consequences for underperformance. This positive approach transformed relationships, particularly between CSOs and teachers. The relationship now focuses on the growth and learning and rests upon mutual accountability whereby both parties are jointly responsible for learning outcomes. This contrasts with previous approaches where CSOs focused on ensuring teachers followed prescribed processes. This shift to focus on learning and teacher improvement played an important role in developing trust but also in enhancing teacher capacity. This positive accountability approach also created a positive feedback loop. Initial successes drove CSO and teacher support, which led to more robust data, which led to greater sharing of successful practices and challenges, which enabled central- and county-level staff to provide more insightful support to schools and CSOs, which led to schools and CSOs wanting to ensure the data they supplied was robust.

Finally, the Tusome programme has been so successful that it has generated trust amongst stakeholders not even involved in programme implementation. While CSOs received training and were equipped with tablets, county-based MOE staff did not participate in the programme. Based on the positive response from CSOs, the MOE staff are requesting training and tablets to help them better support schools.

\section{Reflections on the role of trust, capacity and accountability and the use of EdTech}

Comparing the DLP and Tusome highlights how fostering trust, building capacity and employing accountability mechanisms impacted the effectiveness of EdTech deployment in Kenya. It also shows how appropriately integrating 
EdTech into robust education reform can increase efficiency, enhance effectiveness and support successful education reform. This analysis generated a number of insights that others attempting to harness EdTech may wish to consider while also providing insight into our understanding of the relationship between trust, capacity and accountability.

First, the Kenyan experience demonstrates that when EdTech is integrated into an appropriately designed intervention, it can build system capacity and foster trust between actors by strengthening accountability processes. The Tusome tablets allowed CSOs to collect and share data in real time with teachers, providing insight into student performance to both parties. The live, objective, data provided a foundation for robust pedagogical conversations between teachers and CSOs. Combined with the easy availability of the tools on the tablet, the coach and teacher worked collaboratively to identify ways to address challenges and build teacher capacity. This process fostered trust amongst the parties, leading to more collaborative approaches to enhancing education quality. This easy access to robust data helped facilitate the shift to the new, positive, approach taken to accountability. This focus on improvement rather than punitive consequences enhanced the trust and collaboration between actors. While Tusome focused on pedagogical strengthening, other EdTech solutions can generate similar outcomes. Live attendance monitoring, adaptive education programmes or formative assessment programmes can all be used to generate data and potentially produce similar results in different education service delivery niches.

Second, Kenya's experience demonstrates why EdTech is not a silver bullet that will provide a solution to any and all challenges affecting low-performing education systems. The contrast of the DLP and Tusome highlights how EdTech can support learning when used to enhance efficiency or effectiveness in a specific niche. It also shows that EdTech by itself cannot improve learning outcomes. Rather, interventions integrating EdTech must be appropriately targeted, well designed and robustly implemented to avoid wasting large amounts of money that could be invested in other interventions.

Third, creating trust in an EdTech tool, developing the capacity of users to operate the tool and putting in place an accountability structure to support engagement are all required for EdTech to drive better learning outcomes. In the case of the DLP, a lack of trust, the neglect of capacity building and the lack of an accountability system limited impact. In a system already undergoing upheaval, the lack of focus on building trust in the reform amongst programme implementers meant that the intervention faced challenges from the outset. Inadequate training and a lack of accountability meant that devices went unused resulting in the large investment being wasted. This was in direct contrast to Tusome, where adequate consideration of these elements contributed to successful outcomes.

Fourth, even though technology is playing an ever-increasing role in society, and the general level of trust in and capacity to utilise tech is growing, it is still important to explicitly consider trust and capacity when deploying EdTech. Kenya has been at the forefront of integrating digital solutions into daily life. 
Despite a high level of trust and capacity, inserting digital devices into classrooms under the DLP did not achieve the desired impact. However, under Tusome, where end users were adequately trained in how to use the devices to increase efficiency and effectiveness, a large impact was realised.

Fifth, the planning and implementation of interventions using EdTech tools must be carefully aligned with the local context. The Kenyan education system has been through a long period of intense reform. These reforms required staff to learn new roles, adopt new education approaches and undertake reform-related work in addition to existing duties. Stakeholders did not want further reforms, particularly expensive ones that did not address fundamental infrastructure, training or resource requirements. Tusome complemented the existing reforms, targeting areas where additional support was required.

Finally, it is important to note that trust, capacity and accountability are not static elements, with each new project building on the experiences of those gone by. Tusome fostered trust and capacity in key stakeholders which will make them conducive to EdTech initiatives into the future. Conversely, the poor performance of the DLP has led to distrust amongst education stakeholders. The second phase of the DLP will have to be carefully planned and implemented to overcome this distrust.

\section{Conclusion}

Around the world, policymakers, leaders and entrepreneurs continue to try to harness EdTech to enhance learning. While the development and use of EdTech have grown exponentially, it is yet to consistently demonstrate the outcomes many had expected. Muted or negative impacts often occur when EdTech is pitched as a silver bullet solution to complex, systemic, problems.

While EdTech is not a silver bullet, in Kenya, it has been used to help enhance the efficiency and effectiveness of a well-designed education reform project. This comparison of the DLP and Tusome highlights the importance of taking a sophisticated, holistic, approach to EdTech deployment, and in particular, how important it is to foster trust in interventions, develop the capacity of end users and design and deploy appropriate accountability mechanism.

\section{Note}

1 Commencing in 1995, the SACMEQ is Africa's only regionally equated assessment.

\section{References}

Altinok, N., Angrist, N., \& Patrinos, H. A. (2018). Global Data Set on Education Quality (1965-2015) (No. 8314). Retrieved from http://econ.worldbank.org.

Arias Ortiz, E., \& Cristia,J. (2014). The IDB and Technology in Education: How to Promote Effective Programs? Inter-American Development Bank (July). Retrieved from http:// www.iadb.org.

Barmao, C. (2014). Laptops-for-Pupils Project: Challenges and Opportunities in Public Primary Schools in Eldoret Municipality (Vol. 4). Retrieved from www.iiste.org. 
Batty, R., Wong, A., Florescu, A., Sharples, M., \& Sharples, M. (2019). Nesta: EdTech Testbeds Models for Improving Evidence. London.

Bold, T., Kimenyi, M., Mwabu, G., \& Sandefur, J. (2010). Does Abolishing Fees Reduce School Quality? Evidence from Kenya. Retrieved from https://pdfs.semanticscholar. org/9171/8d541e354bcdc49bd5a092f9f3fe0e6f0313.pdf.

Colclough, C., \& Webb,A. (2010). The Impact of Aid on Education Policy in Kenya (No. 36). Retrieved from www.oecd.org/document/22/0.

Crouch, L., \& Destefano, J. (2015). A Practical Approach to In-Country Systems Research. Retrieved from https://assets.publishing.service.gov.uk/media/57a09dcced915d3cfd001c14/14_Crouch-DeStefano.pdf.

Escueta, M., Quan, V., Nickow, A. J., Oreopoulos, P., Anzelone, C., Balu, R., ... SweetenLopez, O. (2017). Education Technology: An Evidence-Based Review. Retrieved from http://www.nber.org/papers/w23744.

Freudenberger, E., \& Davis, J. (2017). Tusome External Evaluation-Midline Report. Retrieved from https://pdf.usaid.gov/pdf_docs/PA00MS6J.pdf.

Gichura, S. (2003). The Turning Point: Free Primary Education in Kenya (pp. 37-41). In: Critical Perspectives on Education and Skills in Eastern Africa at Basic and Post-Basic Levels. NORRAG News. https://www.norrag.org/fileadmin/Full\%20Versions/NN32.pdf.

Isaacs, S. (2015). A Baseline Study on Technology-Enabled Learning in the African and Mediterranean Countries of the Commonwealth. Retrieved from www.col.org.

Kaffenberger, M., \& Spivack, M. (2019). Not So Fast: Slow Pace of Learning Means Big Changes Are Needed. From RISE Programme website https://www.riseprogramme. org/blog/not_so_fast (Retrieved 22 May 2019).

Kenya National Bureau of Statistics and United Nations Children's Fund. (2011). Kenya Multiple Indicator Cluster Survey 2011, Nyanza Province. Retrieved from https:// microdata.worldbank.org/index.php/catalog/2660/study-description.

Kenyan ICT Authority. (n.d.). Teacher Capacity - DigiSchool - ICT Authority. From http:// icta.go.ke/digischool/teacher-capacity/ (Retrieved 13 April 2019).

Mariga, G., Ogenga, S., Shikali, C., \& Muliaro, J. (2017). Computer laptop project strategy for basic education schools in Kenya. International Journal of Information and Communication Technology Research, 7(5). Retrieved from https:// pdfs.semanticscholar.org/9ac1/3c23157d3fd44e73bb0aa7bbcefc21d98180. pdf?_ga=2.154147832.1750334033.1572288542-1256155719.1572288542.

Mengitsu, A., \& Imende, S. (2013). Kenya's Mobile Tech Revolution - Selamta. From Selamta website https://www.selamtamagazine.com/stories/kenyas-mobile-tech-revolution (Retrieved 28 October 2019).

Ministry of Education. (2018). 2018 Education Quality Dialogues. Retrieved from http:// www.education.go.ke/images/GPE_PRIEDE/2018-FINAL-DIALOGUES-.pdf.

Ministry of Education Science and Technology. (2014). 2014 Basic Education Statistical Booklet. Nairobi.

Mwingirwa, M. I., Marguerite, M. K., \& Khatete, D. (2015). Teachers' Perspectives Towards Use of Technology: the Case of Geogebra in Teaching Mathematics in Kenya. Scientific Research Journal, III(IX), 19-24. Retrieved from http://www.geogebra.org.

Ngugi, M., Mumiukha, C., Fedha, F., \& Ndiga, B. (2015). Universal primary education in Kenya: Advancement and challenges. Journal of Education and Practice, 6(14), pp. 87-95. Retrieved from www.iiste.org.

Nyaga, F. (2018). Assessment of Public Primary School Teachers Preparedness in the Implementation of the Digital Literacy Programme in Public Primary Schools in Imenti North Sub-County Kenya. Retrieved from http://erepository.uonbi. ac.ke/bitstream/handle/11295/104206/FELISTAS NYAGA FINAL PROJECT. pdf?sequence $=1 \&$ is Allowed $=\mathrm{y}$. 
Odero, K. (2016). It's All Systems Go for Kenya's Digital Learning Project as Devices Arrive from China. IAfrikan.com.

Ogle, M. A., Wasanga, P. M., \& Wambua, R. M. (2012). The SACMEQ III Project in Kenya: A Study of the Conditions of Schooling and the Quality of Education. Retrieved from www.knec.ac.ke.

Onsomu, E., Nzomo, J., \& Obiero, C. (2005). The SACMEQ II Project in Kenya: A Study of the Conditions of Schooling and the Quality of Education. In: SACMEQ Educational Policy Research Series. Retrieved from http://www.tzdpg.or.tz/fileadmin/documents/ dpg_internal/dpg_working_groups_clusters/cluster_2/education/3-Core_Documents/ SACMEQ_Tanzania_2005.doc.

Piper, B., Destefano, J., Kinyanjui, E. M., \& Ong'ele, S. (2018). Scaling up successfully: Lessons from Kenya's Tusome national literacy program. Journal of Educational Change, 19(3), 293-321. https://doi.org/10.1007/s10833-018-9325-4.

Piper, B., Oyanga, A., Mejia, J., \& Pouezevara, S. (2017). Implementing large-scale instructional technology in Kenya: Changing instructional practice and developing accountability in a national education system. International Journal of Education and Development Using Information and Communication Technology, 13(3), 57-79. Retrieved from http://ezproxy.lib.uconn.edu/login?url=https://search.ebscohost.com/login. aspx?direct $=$ true $\& \mathrm{db}=$ eric $\& A N=E J 1166622 \&$ site $=$ ehost-live.

RTI International. (2014). USAID/Kenya Primary Math and Reading (PRIMR) Initiative: Final Report. Retrieved from https://globalreadingnetwork.net/sites/default/files/ reading_program_info_files/FR_Kenya.pdf.

Singh, A. (2018). Understanding the EdTech Product Landscape. From Medium website https://medium.com/the-edtech-world/edtech-landscape-743716608675 (Retrieved 3 February 2019).

U.S. Embassy in Kenya. (2016). Tusome and USAID Improve Access to Education. Retrieved from https://ke.usembassy.gov/tusome-usaid-improve-access-to-education/.

UNESCO. (2005). Challenges of Implementing Free Primary Education in Kenya: Assessment Report. Nairobi.

United Nations Population Fund. (2019). World Population Dashboard: Kenya. From https://www.unfpa.org/data/world-population/KE (Retrieved 22 May 2019).

Uwezo. (2015). Are our children learning? The State of Education in Kenya in 2015 and Beyond. Retrieved from http://www.uwezo.net/wp-content/uploads/2016/05/4-pages-uwezoreport2.pdf.

Wanzala, O. (2018, July 25). Laptops lie idle in schools on lack of skilled tutors. Business Daily. Retrieved from https://www.kionjo.com/news/client/desktop/newsarticle/Laptopslie-idle-in-schools-on-lack-of-skilled-tutors/21bb33aa-d44b-4bba-93bf-c32e2c5b010a.

Wanzala, O. (2019, May 16). Magoha tells off critics of new education system - Daily Nation. Daily Nation. Retrieved from https://www.nation.co.ke/news/education/-No-turningback-on-new-curriculum/2643604-5117000-aqwbyhz/index.html.

Wanzala, O., \& Nyamai, F. (2018, July 23). Big hurdles thwart Jubilee's laptops plan. Daily Nation.

Weller, M. (2018). Open Research Online. Educause Review Online, 53(4), 34-48. https://doi. org/10.5860/choice.51-2973.

World Bank. (2019a). Net Official Development Assistance Received (Current US\$). Kenya: Data. From World Bank Open Data website https://data.worldbank.org/indicator/DT.ODA. ODAT.CD?locations=KE (Retrieved 28 October 2019).

World Bank. (2019b). World Development Indicators Database. From World Bank Open Data website https://data.worldbank.org/country/kenya?view=chart (Retrieved 20 February 2019). 


\section{0}

\section{FROM HIERARCHY AND MARKET TO HIERARCHY AND NETWORK GOVERNANCE IN CHILE}

\section{Enhancing accountability, capacity and trust in public education}

\section{Carmen Montecinos, Álvaro González, and Melanie Ehren}

\section{Introduction}

The law that creates the New Public Education System (NEP, Law No 21.040) was introduced in 2017 to change the governance structure for the provision of public education and represents the latest system reform undertaken in Chile, to improve educational quality and equity. Between 2018 and 2025, the NEP legislation will gradually change the intermediate level of the education system by replacing departments and corporations of education operated by municipal governments, currently responsible for administering public schools, with 70 Local Public Education Services (SLEs). In addition, this law creates an institution within the Ministry of Education, the Directorate of Public Education (DEP), with the purpose of centrally coordinating and guiding these SLEs. The main task of these SLEs is to enhance the quality of public education by structuring and organizing support to schools under their jurisdiction. The emphasis on between-school collaboration within each SLE and among the 70 SLEs and the DEP outlines networking as a new mode of governance for the Chilean Public Education System. Network governance is a distinct break from the past two decades of educational reforms, which included a combination of hierarchical and market models.

In this chapter, we examine this system reform to understand how, in practice, trust and capacity are harnessed and/or diminished when network governance is introduced to replace competition in the provision of public education. ${ }^{1}$ This mode of coordination will operate in combination with a managerial approach and hierarchical control by the Ministry of Education through the National System of Quality Assurance of Education. In what follows, we first provide an overview of the previous hierarchical and market governance model, explaining the decline of public education in Chile and the rationale for change in the 
mode of coordination. We highlight three structural weaknesses associated with the provision of public education by municipal departments and corporations of education that underpinned this change. Then, we analyse how, in order to address these weaknesses, NEP legislation maintains 'managerialism' and introduces 'networking' as new mode of coordination. After explaining these coordination mechanisms, we examine the possibilities and tensions arising from their combination to effectively harness trust and capacity to offer high-quality learning opportunities to students attending public schools within an accountability system that retains strong hierarchical and market components.

\section{Market and hierarchical governance of the Chilean education system}

Increasingly Chilean families have perceived public municipal schools as the least attractive option for educating their children. In 2000, over 54\% of elementary and secondary students in Chile were enrolled in municipal schools. By 2014, this number dropped to $36.8 \%$ (OECD, 2017). How can we explain this? To answer this question, we outline key features of the governance of the education system under a combination of hierarchical and market models and its impact on public schools.

\section{Characteristics of market governance in Chile}

In 1981, a large-scale reform introduced by the dictatorship of Augusto Pinochet (1973-1989) transferred the administration of elementary and secondary public schools from the State to municipalities and incentivized private providers to open and run schools. The Ministry of Education (Mineduc) retained centralized control over technical-pedagogical aspects, such as the national curriculum, evaluation and assessment and professional development (Donoso-Díaz et al., 2015). This reform created a quasi-market that fostered competition among private and municipal providers, strengthened parental choice and developed a funding formula based on a State subsidy transferred to municipal and private administrators as a per-pupil, attendance-based voucher (Bellei and Vanni, 2015).

After the restoration of democracy in 1990, successive governments from a centre-left political coalition opted to maintain and enhance the marketization of education. Municipal schools became the only option for many families who were excluded from private-subsidized schools and some selective high-performing municipal secondary schools; these schools increasingly engaged in selection on the basis of academic performance, effectively skimming off the highest performing students (Carrasco et al., 2017; Treviño et al., 2016). Others were excluded through an add-on fee, known as co-payment, charged to parents by private-subsidized schools. These choice and selection mechanisms created a system which is highly segregated by social class, with low-income students concentrating in municipal schools and high-income students going 
to private providers (OECD, 2004, 2017; Valenzuela and Montecinos, 2017). The State aimed to ensure public education by mobilizing school improvement through various measures (Raczynski and Muñoz, 2007), but numerous studies show that by maintaining marketization and developing new policies that incentivized privatization, public education was systematically undermined (OECD, 2004, 2017).

In addition to the high socio-economic segregation, the Chilean school system featured unequal learning opportunities when comparing learning outcomes across private-subsidized and municipal schools (Mizala and Torche, 2012). Students' scores on the SIMCE, a high-stakes census-based achievement test, administered to students in grades 4,8 and 10, are higher in private-subsidized schools, although these differences disappear when controlling for the socio-economic status of students: when comparing student outcomes across quintiles according to income level, test scores increase by the level of income at all grade levels. Thus, private-subsidized schools, in terms of SIMCE results, did not necessarily add academic value (OECD, 2017). Notwithstanding, in Chile, the majority of the population still believes that private-subsidized schools provide a better education (Elacqua et al., 2010).

To address these undesirable outcomes of marketization, a number of structural reforms were implemented between 2008 and 2017 (Valenzuela and Montecinos, 2017). First of all, the Preferential School Subsidy Law of 2008 (SEP, Law No 20.248) provided schools with greater resources per low-income student, contingent upon the development and implementation of a 4-year School Improvement Plan (SIP). Second, the Inclusion Law passed in 2015 (Law No 20.845) prohibited private-subsidized schools to request parents to pay school fees. These schools were also no longer allowed to select students and they no longer received state vouchers if they operated as for-profit institutions. The Inclusion Law also issued a rise in per-pupil funding as a function of the number of low-income students in the school.

\section{Characteristics of hierarchical governance in Chile}

The highly marketized system of the past 20 years also encompassed strong hierarchical control over key school processes and outcomes with various arrangements of vertical accountability (Manroy, 2004). The introduction of the National System of Quality Assurance of Education (SQA, Law No 20.529) in 2011 is a key element in these hierarchical arrangements, consolidating the role of the ministry at the central level and enabling it to control education policy, continuous professional development, technical support to schools and funding of schools via the voucher system. The quality assurance system also features three institutions, independent of the ministry but exercising centralized state control over key aspects of the education system: the National Education Council (CNED), the Quality Agency and the Superintendence of Education. 
The CNED approves the national curriculum, assessment instruments and frameworks for pupils and school professionals, procedures for classifying schools' performance levels and other regulations norming educational institutions. As such it acts as the ultimate arbitrator of school quality. The Superintendence is in charge of financial and administrative audits of schools and their administrators, supervising their compliance with laws and regulations and processing grievance complaints from students' parents and guardians. The Quality Agency is responsible for evaluating pupil learning with standardized tests, school inspection and providing information to guide the educational system towards improving quality and equity. As a key assessment device, SIMCE results are used to classify schools, to sanction low-performing schools and reward high-performing ones, thus creating incentives for schools to improve. These results are also used to target inspections to low-performing schools (labelled 'Insufficient') and to allocate increased support to these schools.

Through the National System of Quality Assurance (SQA), the State, represented by all these institutions, assumed an evaluative position in order to regulate and make up for the flaws and omissions of the market model (Parcerisa and Falabella, 2017). Although SQA legislation purports to ensure schools' quality, questions have been raised about the fairness of the tools used; particularly the punitive nature of accountability through SIMCE has been criticized by organized groups of academics, practitioners and students (Pino-Yancovic et al., 2016). This critique as well as the increasing discontent with the market model led to a substantial restructuring of the public education sector as we explain next.

\section{Structural weaknesses of municipal public education}

Discontent with Chile's education system was particularly focused on the (perceived) lack of quality of public schools, run by municipalities within a system of central control, and the privileged position of private-subsidized schools. Various reforms mentioned earlier (Preferential Schools Subsidy law and Inclusion Law) failed to create a more equal system. Next, we discuss three structural weaknesses in the governance of public education to explain why the hierarchal market model has not led to a more equitable and high-performing system (Bellei, 2018).

\section{Lack of coherent planning and a systemic approach for the governance of public education}

When schools were devolved to municipal governments in the 1980s, administrative support/control (municipalities) and technical-pedagogical control/ support (Mineduc) to schools were separated. Each of the 345 municipalities administering public schools operated as an independent and isolated unit, with low regional coordination. Although the ministry regulates some of the basic conditions of educational quality and efficiency nationally, as described in the previous section (e.g. curriculum, assessment), municipal authorities may have 
had different priorities or lack the capacity to ensure that schools were supported in offering a high level of education.

The lack of the ministry's control over municipalities (see also point 2) and the potential divergence of interests between the two inhibited the impact of several ministerial initiatives that aimed at medium- and long-term goals at the school level. The lack of system coherence could also be observed when the objectives of a school's improvement plan (PME) differed from the goals of the municipality, which, in turn, differed from those proposed by the ministry. For example, where many municipalities often prioritized lowering personnel costs by reducing staff, a school may have called for an increase in the number of teachers to implement a new reading programme coming from the ministry of education (Montecinos et al., 2015).

\section{Low accountability at the municipal level}

Although municipalities were responsible for the quality of their schools, they themselves were not held accountable for their role in supporting school improvement. Vertical accountability mechanisms defined by the National System of Quality Assurance of Education particularly served to pressure schools. For instance, schools classified with Insufficient performance should be closed after 4 years, whereas their municipal administrator faced no sanctions for failing to properly support these schools. Their priorities often also diverged from those of their schools as SAC did not require both to share responsibility for attaining outcomes defined in school improvement plans, even though municipalities controlled the resources schools needed to implement their improvement plan. When funds were insufficient or arrived too late for schools to ensure a good-quality education, they were the ones facing sanctions instead of the municipality.

Municipalities were expected to be held accountable for the lack of school quality in local elections and through citizen voice, but in Chile the demand for quality education has always been directed at the state. A study by UNICEF (2014) shows that when social groups rejected the market model and the commodification of education and called for higher quality education for all, they targeted the Ministry of Education as the agent of change.

\section{Heterogeneous capacities among municipalities to support schools}

With few exceptions, municipalities have been an inefficient manager of educational services, lacking capacity due to factors such as their geographical location (i.e. rural or inner-city urban), their small size (too few schools or low enrolment) and financial constraints brought by the steady decline in enrolment (Donoso-Díaz et al., 2015). Municipalities' financial resources, management and technical-pedagogical capacity to support schools were closely associated with their size and wealth, contributing to high spatial inequalities. 
Small-scale and disjointed operations thus resulted in most municipalities not implementing functions and responsibilities associated with an effective intermediate level (Raczynski et al., 2019). Among the 345 municipalities in Chile, few were able to (1) ensure that each school had access to resources and capacity to provide their students with quality instruction; (2) generate conditions at each school to engage in a process of school improvement; (3) lever the power of networking to foster individual, collective, organizational and inter-organizational learning and innovation within the municipality and (4) generate data and information at the municipal level to engage in systemic improvement. Moreover, within a municipality, high- and low-performing schools coexisted without local capacity to ensure that the latter received needed support. As enrolment determines access to funding and other resources, schools within a municipality competed against each other through various marketing schemes to attract students (Carrasco and Fromm, 2016).

\section{Framing a solution: The New Public Education System}

The NEP aims to address these structural weaknesses by dismantling municipal administration and devolving control to the State which will steer public education through a new structure of SLEs (see Figure 10.1). At the central level, the

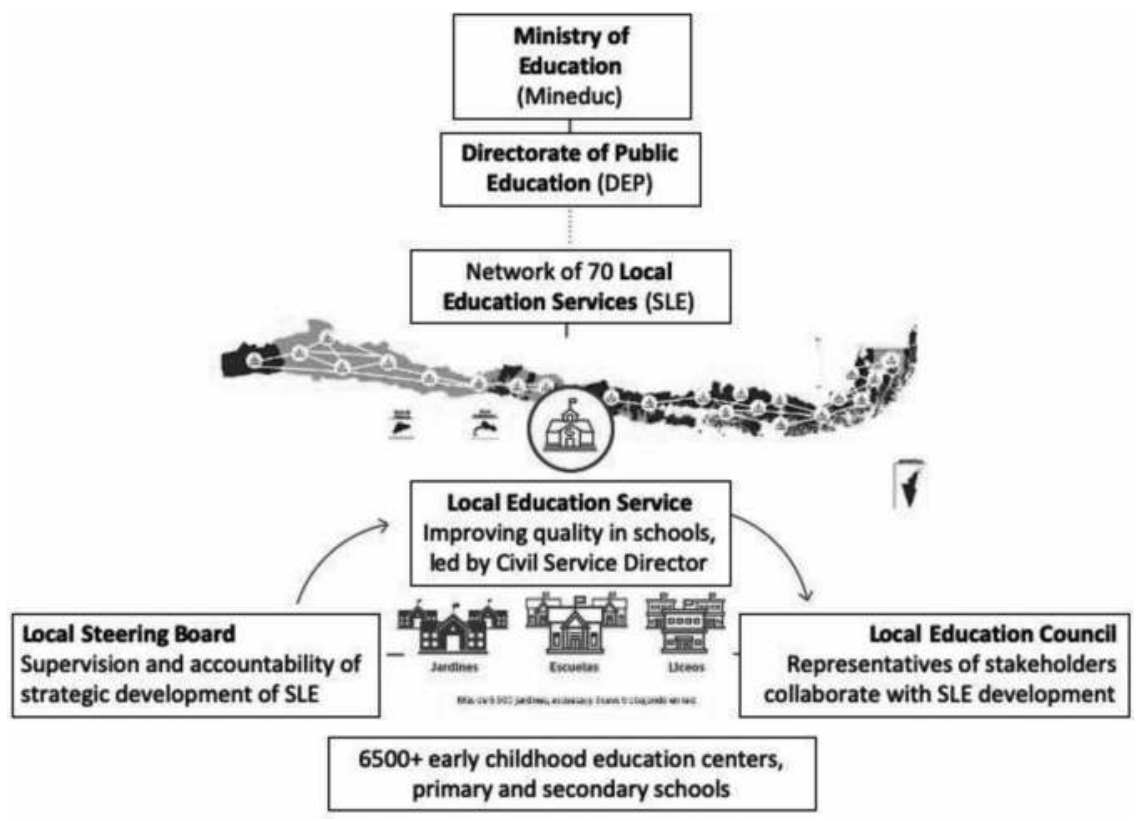

FIGURE 10.1 New public education system

Source: Adapted and translated from https://educacionpublica.cl/organigrama-sistema-2/ (accessed on March 2019). 
DEP steers the system coordinating 70 SLEs. These SLEs are positioned at the intermediate level, each managing between 35 and 140 schools previously under municipal administration, consolidating five municipal departments or corporations of education on average. Each SLE is led by an appointed Civil Service Director with a Local Steering Board and a Local Education Council representing a range of stakeholders of the territory (teachers, parents, local businesses, universities, etc.). Schools and early years centres are the basic units of the system.

The coordination among these three levels, DEP, SLE and schools, is at the centre of NEP's governance model. Our analysis of the NEP Law shows that to accomplish this, two mechanisms were put in place, each with their own set of drivers: managerialism and networking. Managerialism places PME processes as key coordination drivers (Maxcy, 2009). Networking positions trust between partner organizations in the network and their collaboration as the principal method of coordination (Brown and Poortman, 2018). We explain both mechanisms next and then discuss how these are expected to coexist.

\section{Managerialism as a coordination mechanism}

A managerial approach, as characterized by Maxcy (2009), involves an emphasis on professionals who improve productivity (learning outcomes), through effective processes of PME. By providing performance information, derived from the aforementioned processes, power asymmetries between educational service providers and clients are expected to be reduced, while transparency is increased. As pointed out in previous sections, this form of managerialism has been a standing feature of the Chilean school system, as a way for the State to control and regulate the education quasi-market and it is now intensified to coordinate and control the operation of SLEs, the collaboration between schools in a territory and between the SLEs. The NEP legislation introduced a series of performance-management instruments and procedures that are meticulously detailed in the law, aiming to enhance vertical and horizontal accountability, and trust through transparency (see Table 10.1). These instruments specify the responsibilities, roles and rules of coordination among actors involved in the new system in terms of their (1) planning, (2) evaluation and monitoring and (3) participation.

\section{Planning}

Each SLE develops its own Local Strategic Plan (6-year plan) and Annual Plan, and each school develops an Improvement Plan (4-year plan); all of these are developed through participatory processes where the legislation also ensures alignment between both plans. Each SLE Local Strategic Plan should define local goals that integrate national ones (National Strategy developed by DEP), as well as those from each school's Improvement Plan. Each school also develops an Institutional Educational Plan defining the mission, vision and values orienting the collective work within schools. To safeguard decentralization, the person 
208 Carmen Montecinos, Álvaro González, and Melanie Ehren

TABLE 10.1 Summary of planning instruments and their monitoring

\begin{tabular}{|c|c|c|c|}
\hline Law Article & Planning Instrument & Periodicity & Audience \\
\hline 6 & National Strategy & $\begin{array}{l}\text { Every } 2 \text { years progress } \\
\text { report }\end{array}$ & $\begin{array}{l}\text { Education Commission } \\
\text { in Congress } \\
\text { SQA institutions } \\
\text { Ministry of Education } \\
\text { website }\end{array}$ \\
\hline $\begin{array}{l}\text { Transitory } \\
\text { provisions, } \\
\text { article } 7\end{array}$ & $\begin{array}{l}\text { Implementation process } \\
\text { (2018-2025) } \\
\text { Report prepared by the } \\
\text { National Council for } \\
\text { the evaluation of the } \\
\text { Public Education } \\
\text { System }\end{array}$ & Yearly progress report & $\begin{array}{l}\text { President } \\
\text { Education Commission } \\
\text { in Congress }\end{array}$ \\
\hline 11 & SLE Strategic Plan & Yearly progress report & $\begin{array}{l}\text { Annual meeting of } \\
\text { heads of schools and } \\
\text { early childhood centres }\end{array}$ \\
\hline 30 & SLE Strategic Plan & $\begin{array}{l}\text { Progress report as } \\
\text { requested }\end{array}$ & Local Steering Board \\
\hline 46 & Annual Plan & $\begin{array}{l}\text { Progress report as } \\
\text { requested }\end{array}$ & Local Steering Board \\
\hline 8 & $\begin{array}{l}\text { School Improvement } \\
\text { Plan }\end{array}$ & $\begin{array}{l}\text { Yearly participatory } \\
\text { evaluation day }\end{array}$ & $\begin{array}{l}\text { School members } \\
\text { Representative from the } \\
\text { SLE }\end{array}$ \\
\hline 10 & $\begin{array}{l}\text { School Management- } \\
\text { School Principal }\end{array}$ & Yearly & $\begin{array}{l}\text { School members } \\
\text { SLE director or } \\
\text { representative }\end{array}$ \\
\hline 22 & SLE Annual Plan & Yearly & $\begin{array}{l}\text { Open meeting } \\
\text { Publish in SLE website } \\
\text { Local Steering Board }\end{array}$ \\
\hline 23 & $\begin{array}{l}\text { Categorization by } \\
\text { Quality Agency }\end{array}$ & $\begin{array}{l}\text { Any time a school is } \\
\text { categorized as } \\
\text { Insufficient by the } \\
\text { Agency }\end{array}$ & $\begin{array}{l}\text { Director of Public } \\
\text { Education } \\
\text { SLE Local Council }\end{array}$ \\
\hline 37 & $\begin{array}{l}\text { Meetings of the Local } \\
\text { Steering Board }\end{array}$ & Every meeting & Publish in SLE website \\
\hline 41 & $\begin{array}{l}\text { SLE Director } \\
\text { Performance } \\
\text { Agreement }\end{array}$ & Yearly progress & $\begin{array}{l}\text { Director of Public } \\
\text { Education } \\
\text { Publish in SLE website }\end{array}$ \\
\hline 52 & $\begin{array}{l}\text { Assessment report of } \\
\text { schools' results - } \\
\text { Quality Agency }\end{array}$ & As requested & Local Council \\
\hline 52 & $\begin{array}{l}\text { SLE Inspection } \\
\text { Report - Quality } \\
\text { Agency }\end{array}$ & As requested & Local Council \\
\hline
\end{tabular}


TABLE 10.1 Summary of planning instruments and their monitoring (continued)

\begin{tabular}{|c|c|c|c|}
\hline Law Article & Planning Instrument & Periodicity & Audience \\
\hline 57 & $\begin{array}{l}\text { Meetings of the Local } \\
\text { SLE Council }\end{array}$ & Every meeting & Publish in SLE website \\
\hline 61 & $\begin{array}{l}\text { State of Public } \\
\text { Education - DEP } \\
\text { Director }\end{array}$ & Yearly & N/A \\
\hline 82 & $\begin{array}{l}\text { Holistic evaluation of } \\
\text { the Functioning of } \\
\text { SLE - Quality Agency }\end{array}$ & $\begin{array}{l}\text { At least every } 6 \text { years } \\
\text { or more frequently } \\
\text { based on the number } \\
\text { of schools categorized } \\
\text { as Insufficient }\end{array}$ & $\begin{array}{l}\text { Director of Public } \\
\text { Education } \\
\text { Local Steering Board } \\
\text { Local Council }\end{array}$ \\
\hline
\end{tabular}

Source: Authors' own elaboration, based on Law No 21.040.

responsible for managing each of these plans has a certain level of autonomy to define goals and activities coupled with some level of discretion as to whether to use the feedback from advisory groups or upper level line managers.

These planning instruments (National Strategy, SLE Strategic Plan, SLE Annual Plan and School Improvement Plan) share a number of characteristics of a managerial approach, particularly by aligning the goal-setting and subsequent planning among them. First, planning is limited by the funding available, but each partner is afforded relative autonomy in deciding on the type and level of investment needed to improve the quality of schools. Second, by aligning goals and strategies across these plans and widely publicizing them through institutional websites, citizens know the outcomes against which to judge the performance of public education. Third, a result-oriented system enables policymakers and practitioners to develop a monitoring and evaluation system to orient decision-making. Fourth, these plans integrate administrative, financial and pedagogical resources and actions that mutually support each other. For example, the law is very specific in requiring each SLE to include a provision of support of failing schools in their annual plan and an SLE director can be dismissed if the number of school deemed of Insufficient performance in his/her jurisdiction does not decrease in line with the goals stipulated in the annual plan.

This managerial approach to planning is further evidenced by performancemanagement agreements through which the head of the various administrative units will be yearly assessed: SLE director, SLE deputy directors and school principals. They will be individually evaluated against progress towards the goals in their respective plans and leaders across the various levels know what they and others must account for and work towards in an effort to make their SLE locally, and NEP nationally, coherent and effective. SLE directors' agreements are negotiated between the SLE Board and the DEP director, and the SLE director defines agreements for school principals. The evaluation and monitoring, 
a second element of a managerial approach, further aim to improve the planning for improvement and building of trust across the SLE, as we outline next.

\section{Evaluation and monitoring}

Evaluation and monitoring of both schools and the SLE are also regulated in the NEP. The legislation puts in place multiple controls for the performance of SLEs, in order to enhance accountability, in terms of responsibility for results, and trust, in terms of transparency of information to the community. Three distinct strategies are to be implemented: external evaluation through the Quality Agency, internal monitoring through performance management and public reporting.

External evaluation through the Quality Agency. Accountability as regulated in the NEP builds on the existing hierarchical accountability exercised through the National Quality Assurance System (SAC) but further integrates the various measures in the system, particularly the national standardized testing of student outcomes in the SIMCE test, school inspections with subsequent publication of reports and the categorization of schools by the Quality Agency. These are no longer stand-alone instruments, but their outcomes must be considered in the implementation of performance-management instruments described earlier. Moreover, the Quality Agency is charged with inspecting each SLE at least once every 6 years. ${ }^{2}$ Unchanged is the publication of league tables of individual schools' performance to inform parents' choice, motivate schools with lower performance to either improve their scores or close if failure persists over time. In other words, national tests (SIMCE) and inspections of individual schools will continue to be used to place pressure on schools to raise standards but now this pressure extends to the SLE as they are required to act on the evaluation provided by the Quality Agency.

Internal monitoring through performance management. As shown in Table 10.1, there are a number of articles in the NEP legislation which specify the monitoring of the planning instruments described earlier. Monitoring is done through yearly progress reports of outcomes that should be known, and in some instances approved, by the various entities designed to provide oversight as well as by the school communities. These various audiences of reports range from the President of Chile (yearly progress through the implementation phase of NEP), to the legislature (DEP's National Strategy), local boards and school communities (SLE Strategic and Annual Plans), to a school's council (School Improvement Plan). The implementation of these managerial mechanisms is mostly focused on providing information to understand where adjustments to strategies are needed, but they can also be used to make decisions about the employment of SLE and school leaders.

Transparency through public reporting. NEP legislation introduces several mechanisms for greater transparency and direct access to data on schools' and SLEs' performance. Attention focuses mainly on ensuring wide publicity of SLE- and school-level goals but also includes reporting progress of the DEP's National Strategy. Furthermore, information about the planning, performance agreements and board's and council's decisions are available on a website. 


\section{Participation}

Creating structured spaces that enable different stakeholders to present their views during planning and evaluation seeks to ensure (1) actual transparency in how schools are functioning, (2) the quality they are offering and (3) responsiveness to their concerns throughout the planning and evaluation. Participation allows trust to be built and restored as a condition of successful collaboration within the SLE as well as to incentivize parents to choose public schools over private ones (Smith and Benavot, 2019). Participation, understood as the right to express educational stakeholders' opinions on matters that directly affect them, is a principle of the NEP. Participation is promoted through representative boards and councils and direct involvement through consultation.

Advisory boards and councils. The composition of boards and councils provides for a mechanism to ensure collaboration and articulation among different social services, educational levels and stakeholders within the SLE. The Regional Coordinating Committee includes representatives from a broad range of institutions (i.e. universities, health, SLE, SAC system, regional government offices) and twice a year by the region's governor convenes a meeting. These meetings aim to ensure harmonization between the SLE Strategic Plan and the Regional Development Strategy.

As Figure 10.1 outlines, each SLE has two additional boards: the Local Steering Board and the Local Council. The Local Steering Board (five or six people) represents parents, municipal authorities and regional authorities. Its main responsibilities include proposing to the President of Chile a list of three candidates to appoint the SLE director and request to the DEP director the removal of an incumbent SLE director, approving the Strategic Plan, offer non-binding recommendations for the Annual Plan and providing advice and holding the SLE director accountable for progress in the implementation of these plans.

The Local Council (12 people) is composed of representatives from various constituents (students, teachers, parents, senior school leaders, school councils and tertiary education institutions). Their role is to collaborate with SLE directors by bringing to their attention the needs and opinions of these constituents. The SLE director may seek their advice on matters he or she deems pertinent. The Local Council is also asked to support in the provision of student, parent/guardian and school councils for each school; the latter being constituted of advisors or other stakeholders who support in the development and implementation of the school improvement plan. Additionally, each school has its own advisory council with decision-making power regarding the approval of the school calendar and internal norms and regulations.

Direct consultation with various stakeholders. These various boards and councils all have their role in planning and evaluation, either at the school or SLE level. The NEP regulation, for example, requires the Ministry to consult with school communities when developing the national strategy, while the SLE director is required to convene a meeting with all school principals at least once a year to 
consult them on the implementation of the SLE strategic plan, suggesting areas of improvement. Although these consultations are non-binding, there is an expectation that stakeholders' opinions and proposals are taken into consideration in planning and goal setting.

\section{Networking as a coordination mechanism}

As the previous section outlined, networking, in addition to the management of performance, is one of the key principles of NEP (Art. 5, e and Art. 14) and a key strategy for enhancing school and system capacity for improvement. A networking approach implies a set of policies and arrangements that links a group of three or more legally autonomous organizations around a purpose and set of joint goals (Mayne et al., 2003). This approach positions trust between partner organizations in the network and their collaboration as the principal method of coordination, mobilizing knowledge creation and exchange to increase individual and collective capacity (Brown and Poortman, 2018).

Whereas managerialism has been part and parcel of Chile's educational sector over the last 40 years, NEP's introduction of networking represents a break from the past where schools competed over students in a highly marketized system. In the past, networks only functioned as part of a hierarchical structure where their use was to communicate ministerial policies from the top down (Pino-Yancovic et al., 2020). This legacy is to some extent still present in the NEP as the SLEs are not building on local forms of collaboration or bottom-up initiatives but are mandating the formation of networks from the top down.

To understand the rationale behind the decision to incorporate networking, while at the same time intensifying performance management, we interviewed three key policymakers involved in drafting the NEP law. ${ }^{3}$ Our analysis of these interviews suggests that the introduction of networking aimed to differentiate NEP form municipal governance and generate a more collaborative approach to system-wide improvement.

Networking as a governance mode. From these policymakers' perspectives, the introduction of networking as a principle relates to how the new system will organize and function to enhance schools' quality and improvement. In their views, networks are better able to acknowledge and/or organize the complexities of a Public Education System:

Not only is it [networks] a work modality, it is a vision of governance that is different, it has a touch, an accent, a more administrative emphasis, regarding how we are going to organize schools because we want to generate local systems, we want to generate an ecology that didn't exist before. So, I believe that beyond a strategy that one can implement, this has a foundation in governance, that is, we will structure the institutional framework so that it works that way. [...] where attributions exist and where there is a certain mechanism that makes this [public education system] work best (Policymaker 3). 
The introduction of networks in the restructuring of the public system was also a politically convenient compromise. During the legislative process, there was resistance in congress as some members viewed the NEP as an attempt to centralize decision-making to the state and undermining the autonomy of schools. Networking provided a technical solution for this political problem by distributing more decision-making power to the local level, now well-funded and with management and technical-pedagogical capacity to support schools:

The general idea that is behind the design of the law is an articulated, professionalized system, unlike the municipal system that is a system that is diagnosed as low professionalization and high atomization and disarticulation [...] in this institutional framework they [SLE] will have an opportunity for monitoring and follow-up, infinitely superior and on a scale that the municipal system will never be able to offer (Policymaker 2).

The network approach to improvement also extends to the national level where the DEP is in charge of promoting and facilitating collaboration between the SLEs to ensure their development and continuous improvement. The Directorate is expected to create robust structures to support the SLEs and improve the planning and collaboration across the system, thereby addressing some of the key problems of the lack of coherent planning, atomization and diffusion of responsibilities in the previous system.

Networking as a capacity building strategy. The NEP law indicates that the SLE will be responsible for strengthening and developing the capacities of schools to improve their education. Networking is considered to be a key strategy through which SLE's can meet this expectation ${ }^{4}$ by first of all working on a larger scale in providing support and sharing resources and, second, by exchanging good practices in the collaboration between schools in a territory. As one of the interviewed policy-makers explained, the larger scale of the SLEs is expected to create more capacity for improvement; SLEs can work more efficiently in developing a support package for a larger number of schools instead of, as in the past system, for a few schools in one municipality:

One of the structural deficits, so to speak, of the municipalized system is precisely that it does not have the capacity to develop capacities or to attract them. [...] For instance, the [name] municipality will never be able to offer to its schools a specialist in mathematics in a permanent, systematic way, but the local service could do it, and it could do it because it has the linkage at national level and having a slightly larger scale and being set in an urban center has the ability to attract better professionals, generating a virtuous cycle that should be exploited (Policymaker 1).

Capacity is also built through the collaboration between schools in an SLE, as mandated under the NEP legislation. The Act requires the SLE to develop a strategic 
plan in which it sets out how it builds on existing networks in the territory and structures the collaboration, information sharing and joint decision-making around professional development, access to services and facilities, pedagogy and management of schools. The SLEs central office should decide on the type of collaboration between schools in the territory, such as by grade level, geographical proximity or how schools are assessed by the Quality Agency. Different actors (e.g. school leaders, teachers of language arts) can be asked to join in working groups where each group is expected to address school- or territory-specific issues and needs, such as school climate or truancy. The SLE is also expected to reach out to other organizations, such as healthcare providers, sports clubs or even private schools where relevant.

Tensions and Possibilities of Combining Managerial and Networking Mechanisms to Enhance Capacity and Trust within the NEP.

The predominantly managerial approach to coordinate NEP through strategic planning, performance monitoring and evaluation, vertical and horizontal lines of accountability create an interesting conundrum given the logic of trustful and collaborative relationships underpinning network governance and the hierarchical approach inherent to performance management. Both trust and collaboration are, in the field of public administration, seen as key conditions for more localized decision-making and flexibility in meeting local needs. How does the NEP law create conditions for schools and SLEs to develop the required trust to drive constructive collaboration at the local level? Here we first discuss potential opportunities and risks in relation to the two underpinning principles of the NEP: (1) alignment of goals and subsequent evaluation and reporting within the SLE, and (2) participatory processes of goal setting and evaluation. Then, we analyse how likely these principles are to become reality given the existing pressure from external accountability and the past structure and mindsets of competition and compliance.

\section{Alignment of goals}

As we explained in this chapter, the NEP legislation includes a set of requirements for managers at different levels of the system to become accountable for attaining a set of interconnected targets. These targets structure and incentivize collaboration between the various actors as each benefits from the other's achievement: SLE directors will only be able to meet their targets and receive a positive evaluation if their senior staff at the SLE as well as school principals down the line also meet their own performance targets. This interconnected approach in how performance is managed across the SLE essentially creates a system of reciprocal accountability around a set of joint goals, enabling the various actors to come to a common and shared understanding of values and objectives about high-quality education in the territory. Working towards a set of shared values and objectives across schools within the SLE, particularly when these objectives are developed through a participatory process, provides fertile ground for the development of interpersonal and inter-organizational trust that underpins capacity building within a model of network governance. 
These provisions of aligning goals across the hierarchical levels and ensuring a participatory process of goal setting allow for trust to build across the SLE, even within a managerial approach which is generally more top down and rational. Trust can also be purposefully managed as part of the hierarchical steering: including stakeholders in a local education council provides them with agency in improving education across their territory, it creates buy-in for the improvement goals set in the various plans and strategies and also allows them to hold the various administrative units (SLE director, deputy directors and principals) to account. The alignment of goals in the school, SLE and national strategy could further allow for trust to build by clarifying the various roles and responsibilities across the SLE and ensuring these are oriented towards the same set of outcomes and activities, as some research has found in decentralized systems (Ehren et al., 2017; Janssens and Ehren, 2016). Furthermore, the specific provisions in the NEP legislation to support failing schools (i.e. those judged by the Quality Agency as 'insufficient'), where SLE directors are held accountable for improving these schools, are expected in the rebuild parents' trust in public education and act as a positive lever for their involvement in schools and local education councils.

The availability of information on the attainment of goals, both at the school and SLE level, is another important mechanism to build trust. It allows partners (e.g. schools working with an SLE director and also parents choosing a school) to understand each other's competences in meeting these goals while enabling the alignment of their values and expectations. Transparency and information, however, only enable trust building when data are used and confirm positive expectations of the other's performance, allow partners to use it for benevolent purposes and are collected and used in a fair and just manner. Mills and Rubinstein Reiss (2017) and Barrera et al. (2015), for example, explain how formal control can build trust by providing objective measures and rules that lead to a track record of compliance to be used in future trust evaluations. Barrera et al. (2015) further point out how monitoring can build trust as trust of a trustor increases with her/his possibility of informing other trustors about the behaviour of the trustee, or with the expected duration of a series of interactions between the trustor and trustee in the future. For monitoring to be used in an evaluation of someone's trustworthiness, it needs to be transparent and informative. The multitude of plans and reports required by the NEP legislation (see Table 10.1) suggests that partners in the exchange potentially face an information overload where, even though the information is available, it does not inform the relationship or enable the various actors to constructively work towards the improvement on a set of joined outcomes.

Another potential risk lies in the ability of stakeholders to reach consensus on goals for the various strategies. As the IDB-LAPOP 5 (2016-2017) survey indicates, $64 \%$ of the respondents in Chile stated that they do not trust the political parties and 34\% failed to trust their own communities. Given the low levels of trust within the wider Chilean society, it might be difficult to reach such a consensus among groups with different set of interests and who might be suspicious to one another. 


\section{Participation}

A second principle underlying the performance management in the NEP is the participation of stakeholders in the network in setting goals for both schools' and SLE's strategic plans. Participation in decision-making is an important strategy to build trust as it creates agency among those involved to work collaboratively on improvement, where the collaboration itself can help in aligning values of those involved and creating a sense of shared responsibility for learning outcomes of pupils across the SLE. Involving stakeholders in the decision-making enables them to understand the challenges and issues that need to be addressed from the viewpoint of all involved. Having an opportunity to take someone else's perspective endorses a more positive viewpoint to the other's perspective, which generates trust across the network (Williams, 2012). It also builds capacity, such as when school principals better understand the challenges that teachers are facing in the classroom, or when SLE directors learn which school or school principal needs their support. Williams (2012) describes how such 'perspective-taking' not only fosters understanding and caring actions that build social bonds but also strengthens the trustworthiness of perspective takers in three ways: (1) by motivating them to engage in benevolent actions, (2) by fostering goal alignment, and (3) by enabling them to have a positive emotional influence on others.

A key consideration is that participation is genuine and not just a formality where stakeholders are consulted on set points in time, but where their concerns are taken into account and those involved are listened to. Genuine participation and the involvement of stakeholders can be a difficult process, particularly when there are significant divergences of views and interests among the relevant stakeholders. Hooge and Helderman (2008) talk about how such diversity of views makes joint decision-making and policymaking particularly challenging.

Given the past 40 years of operating within a market model, providing school constituents with greater information can lead to monitoring and disciplining providers, rather than promote innovation and a growth-mindset (Mizala et al., 2007). The functioning and effectiveness of the Local Steering Boards and the extent to which they will be able to collaborate on the basis of trust are contingent on the ability and willingness of the different groups to work together to reach a consensus. As Brown and Poortman (2018) outline, networks can only be successful in improving education when those who are part of the network (parents, teachers, school principals and SLE staff) have the skills and mindsets to collaborate. In the Chilean context, where teachers and principals are accustomed to competition and hierarchical supervision and control, they need to learn how to negotiate a set of shared purposes, develop a sense of shared responsibilities for learners' outcomes across schools and how to create structures to support these. Greany and Higham's (2018) study of Multi-Academy Trusts in England highlights the challenges in making such a transition: school principals in their study, for example, initially wanted to protect the 'intellectual property' of their school and did not want to share resources or expertise with 
other schools out of fear for losing their competitive advantage. Those who are in charge of a network (i.e. the SLE directors in Chile) need to develop new skills in leading and managing change across a network of schools; a skill that is distinctly different from the leadership of a single school.

Teachers and principals will only trust the new structure of working collaboratively in an SLE when their opinions are taken into account in formal planning and evaluation. In the initial evidence regarding the implementation of the first four SLE's, teachers noted that these structures for participation did not seem to have a real impact on decision-making (Uribe et al., 2019). Moreover, as noted by the authors of that evaluation study, concerns over administrative aspects have distracted attention away from building structures for participation and from building a shared vision on teaching and learning.

Other risk around participation as a strategy to build trust and capacity and ensure reciprocal accountability is that the NEP legislation only allows stakeholders, such as parents, to advice and inform on the strategic plans but not to make decisions. López et al. (2012) provide evidence from the Chilean school system indicating that if parents perceive their role as only a formality, their trust in the school (and in this case, the SLE) may be eroded and they are likely going to withdraw their involvement or not become involved at all.

Furthermore, given the existing structure of Chile's education system with public, private and private-subsidized schools, SLEs tend to have a large proportion of high-poverty schools in their network. These schools often perform less well in inspections, league tables and on the SIMCE test. Under the continuing national system of quality assurance (SAC), these schools, when assessed 'insufficient', have to be inspected every 2 years and the report is published online. This creates intense pressure to improve in a short period of time. SLE staff have to ensure the improvement of these schools within 4 years to prevent the school from being closed, while the SLE director might lose his/her job when too many schools are judged 'Insufficient'. The punitive nature of the external accountability and strict time span for improvement allows little time and flexibility for the involvement of stakeholders in decision-making and improvement and little opportunities to rebuild or build trust. Where SLEs need to support schools judged 'insufficient' to improve quickly, they might turn to more centralized approaches to direct school improvement, as we have seen in similar examples of Multi-Academy Trusts in England (Ehren and Godfrey, 2017). This could result in a changed structure of more top down hierarchical steering and control instead of lateral collaboration which might persist after schools come out of the 'Insufficient' category.

\section{Conclusion}

The change in governance structure of public education that we have examined in this chapter is part of a broader programme of reforms aimed at creating a more equitable and inclusive education system. A whole of systems approach 
is required to align roles and responsibilities across the system, improving efficiency as well as reducing potential overlap or conflict (Burns and Köster, 2016). Reforms must resolve tensions between potentially conflicting forces such as accountability and trust, and consensus building within a complex system. In the case of Chile, a sequential approach to governance reforms first introduced changes to regulate private provision (Inclusion Law in 2015) and a reform later addressed public provision (NEP Law in 2017). Implementing in parallel these two reforms presents important challenges to the extent that there is a lack of synergy between them (Muñoz and Weinstein, 2019). For example, private sector regulations may increase the flight of families to private-subsidized schools as entry barriers such as co-payment and selection have been removed (Valenzuela and Montecinos, 2017), while simultaneously NEP aims to increase public schools' enrolment.

The introduction of networking to promote a new and distinctive mode of coordination, combined with a managerial approach to educational improvement, presents both possibilities and tensions for enhancing trust and capacity in public education. By mandating networks, NEP creates external structures to motivate and enable schools to collaborate (Armstrong and Ainscow, 2018; Ehren and Perryman, 2018; Feys and Devos, 2015). External conditions, however, are not enough as internal conditions such as trust between network members, structures for collaboration and knowledge mobilization, skilled leadership to facilitate interactions and broker resources are needed to ensure that networks are an effective strategy to improve education system-wide (Brown and Poortman, 2018; Díaz-Gibson et al., 2017). These internal conditions will need to develop overtime.

As NEP is implemented, addressing potential ways in which managerialism may enhance the leverage of transparency and trust through horizontal accountability and as well as undermine school-to-school collaboration is paramount considering findings from an initial evaluation of the first year of implementation of the first four SLEs (Uribe et al., 2019). Overall, school principals favoured the creation of this new intermediate level, perceiving it as an opportunity to improve public education. Although teachers have been less involved than principals in the implementation process, they also expressed the expectation of receiving greater support from this level. They understood that during the first year, these expectations probably would not be met but they also noted that moving forwards they did expect to receive what NEP promised.

\section{Notes}

1 Funding: This chapter draws data from a larger study supported by the Comision Nacional de Investigación Científica y Tecnológica [CONICYT Grant FB003].

2 An aspect of SAC, explicitly addressed by Law No 21.040 but not addressed in the current chapter, is the role of the Superintendence of Education in the oversight of the correct use of financial resources and compliance with various laws governing the education sector. 
3 Policymaker 1 was a high-rank ministry advisor, especially appointed to lead the legislative discussion of the reform; Policymaker 2 was a senior ministry official, in charge of coordinating and leading professional teams designing the reform; and Policymaker 3 was an expert consultant, leading the archival and literature search to develop minutes, memos and working papers to support the legislative discussion of the law. These interviews were conducted in the context of a larger exploratory case study investigating how two of the first four SLEs to begin operating in 2018 organized inter-organizational networks in their formation stage.

4 Direct support to schools is another strategy, particularly for school classified as Insufficient by the Quality Agency.

5 "How does trust impact your quality of life?" Interamerican Development Bank (IDB) website. Retrieved from https://www.iadb.org/en/improvinglives/how-does-trustimpact-your-quality-life.

\section{References}

Armstrong, P., \& Ainscow, M. (2018). School-to-school support within a competitive education system: Views from the inside. School Effectiveness and School Improvement, 29(4), 614-633.

Bellei, C. (Ed.) (2018). Nueva Educación Pública contexto, contenidos y perspectivas de la desmunicipalización. Santiago: Universidad de Chile.

Bellei, C., \& Vanni, X. (2015). Chile: The Evolution of Educational Policy, 1980-2014 (pp. 179-200). In: S. Schwartzman (Ed.), Education in South America: Education around the World. London: Bloomsbury Academic.

Brown, C., \& Poortman, C. L. (2018). Networks for Learning: Effective Collaboration for Teacher, School and System Improvement. London: Routledge.

Burns, T., \& Köster, F. (Eds.) (2016). Governing Education in a Complex World. Paris: OECD Publishing.

Carrasco, A., \& Fromm, G. (2016). How local market pressures shape leadership practices: Evidence from Chile. Journal of Educational Administration and History, 48(4), 290-308.

Carrasco, A., Gutiérrez, G., \& Flores, C. (2017). Failed regulations and school composition: Selective admission practices in Chilean primary schools. Journal of Education Policy, 48(4), 290-308. https://doi.org/10.1080/02680939.2017.1312549.

Díaz-Gibson, J., Zaragoza, M. C., Daly, A.J., Mayayo, J. L., \& Romaní,J. R. (2017). Networked leadership in Educational Collaborative Networks. Educational Management Administration and Leadership, 45(6), 1040-1059.

Donoso-Díaz, S., Castro-Paredes, M., Alarcón-Leiva, J., \& Davis-Toledo, G. (2015). Nudos críticos del sistema escolar chileno, hipótesis explicativas y propuestas para una nueva arquitectura del sector público. Ensaio: Avaliação e Políticas Públicas Em Educação, 23(86), 37-71.

Ehren, M. C. M., \& Godfrey, D. (2017). External accountability of collaborative arrangements; a case study of a Multi Academy Trust in England. Educational Assessment, Evaluation and Accountability, 29(4), 339-362.

Ehren, M. C. M., \& Perryman, J. (2018). Accountability of school networks: Who is accountable to whom and for what? Educational Management Administration and Leadership, 46(6), 942-959.

Elacqua, G., Martínez, M., \& Aninat, C. (2010). ¿Cómo fortalecer la educación municipal? Capacidad y responsabilidad política (pp. 101-130). In: S. Martinic \& G. Elacqua (Eds.), ¿Fin del ciclo? Cambio en la gobernanza del sistema educativo. Santiago: UNESCO-Pontificia Universidad Católica. 
Feys, E., \& Devos, G. (2015). What comes out of incentivized collaboration: A qualitative analysis of eight Flemish school networks. Educational Management Administration and Leadership, 43(5), 738-754.

Greany, T., \& Higham, R. (2018). Hierarchy, Markets and Networks. Analysing the 'SelfImproving School-Led System' Agenda in England and the Implications for Schools. Available at http://www.lcll.org.uk/uploads/2/1/4/7/21470046/hierarchy-marketsand-networks.pdf.

Janssens, F. J., \& Ehren, M. C. M. (2016). Toward a model of school inspections in a polycentric system. Evaluation and Program Planning, 56, 88-98.

Manroy, C. (2004). Regulation and Inequalities in European Education Systems. Louvain: Université Catholique de Louvain (GIRSEF).

Maxcy, B. (2009). New public management and district reform: Managerialism and deflection of local leadership in a Texas school district. Urban Education, 44(5), 489-521.

Mayne, J., Wileman, T., \& Leeuw, F. (2003). Networks and Partnering Arrangements: New Challenges for Evaluation and Auditing. In: A. Gray, B. Jenkins, F. Leeuw, \& J. Mayne (Eds.), Collaboration in Public Services: The Challenge for Evaluation. New Brunswick, NJ: Transaction Publishers.

Mizala, A., Romaguera, P., \& Urquiola, M. (2007). Socioeconomic status or noise? Tradeoffs in the generation of school quality information. Journal of Development Economics, 84(1), $61-75$.

Mizala, A., \& Torche, F. (2012). Bringing the schools back in: The stratification of educational achievement in the Chilean voucher system. International Journal of Educational Development, 32(1), 132-144.

Montecinos, C., Ahumada, L., Galdames, S., Campos, F., \& Leiva, M.V. (2015).Targets, threats and (dis)trust:The managerial troika for public school principals in Chile. Education Policy Analysis Archives, 23(0), 87.

Muñoz, G., \& Weinstein, J. (2019). The Difficult Process in Chile: Redefining the Rules of the Game for Subsidized Private Rducation (pp. 72-100). In: C. Ornelas (Ed.), Politics of Education in Latin America. Leiden: Brill/Sense.

López, V., Madrid, R., \& Sisto, V. (2012). "Red Light" in Chile: Parents Participating as Consumers of Education under Global Neoliberal Policies. In: H. Cuadra-Montiel (Ed.), Globalization - Education and Management Agendas. London, UK: IntechOpen Limited (pp. 45-74). In Tech. https://doi.org/10.5772/50305.

OECD (2004). Reviews of National Policies for Education: Chile. Paris: OECD Publishing.

OECD (2017). Education in Chile. Reviews of National Policies for Education. Paris: OECD Publishing.

Parcerisa, L., \& Falabella, A. (2017). The consolidation of the evaluative state through accountability policies: Trajectory, enactment and tensions in the Chilean education system. Education Policy Analysis Archives. [S.1.], v. 25, p. 89. Available at https://epaa.asu.edu/ ojs/article/view/3177.

Pino-Yancovic, M., Oyarzún-Vargas, G., \& Salinas-Barrios, I. (2016). Crítica a la rendición de cuentas: Narrativa de resistencia al sistema de evaluación en Chile. Cadernos CEDES, 36(100), 337-354.

Pino-Yancovic, M., González, Á., Ahumada Figueroa, L., \& Chapman, C. (2020). School Improvement Networks and Collaborative Inquiry: Fostering Systemic Change in Challenging Contexts. Bingley: Emerald Publishing.

Raczynski, D., \& Muñoz, G. (2007). The Chilean Educational Reform:The Intricate Balance between a Macro and Micro Policy. In: W. T. Pink \& G. W. Noblit (Eds.), International Handbook of Urban Education (Volume 19, pp. 641-664). The Netherlands: Springer. 
Raczynski, D., Rivero, R., \& Yañez,T. (2019). Nivel intermedio del sistema escolar en Chile: Normativa y visión de los sostenedores sobre funciones, preparación para el cargo y las prácticas. Calidad en la Educación, 51, 382-420.

Smith, W. C., \& Benavot, A. (2019). Improving accountability in education:The importance of structured democratic voice. Asia Pacific Education Review, 20, 193-205.

Treviño, E.,Valenzuela,J. P., \&Villalobos, C. (2016).Within-school segregation in the Chilean school system: What factors explain it? How efficient is this practice for fostering student achievement and equity? Learning and Individual Differences, 51, 367-375.

UNICEF (2014). La voz del movimiento estudiantil 2011. Educación pública, gratuita y de calidad. Santiago, Chile: Fondo de las Naciones Unidas para la Infancia.

Uribe, M., Valenzuela, J. P., Anderson, S., Cuglievan, G., Núñez, I. M., \& Hernández, C. (2019). Estudio de seguimiento y sistematización de procesos críticos en la transición de la gestión de la educación pública de los municipios a los Servicios Locales de Educación (SLE).Valparaíso, Chile: Centro Líderes Educativos Pontificia Universidad Católica de Valparaíso; CIAE Universidad de Chile.

Valenzuela, J. P., \& Montecinos, C. (2017). Structural Reforms and Equity in Chilean Schools. Oxford Research Encyclopedia of Education (Volume 1). Oxford University Press. https://doi. org/10.1093/acrefore/9780190264093.013.108. 


\section{1}

\section{CONTRASTING APPROACHES, COMPARABLE EFFICACY?}

\section{How macro-level trust influences teacher accountability in Finland and Singapore}

\section{Yue-Yi Hwa}

\section{Introduction}

On today's educational stage, Finland and Singapore cast outsized shadows. Since gaining the spotlight with standout performances in PISA and TIMSS, these relatively small countries - each weighing in at roughly 5.5 million people (United Nations, 2017) - have featured in policy discussions and media reports worldwide. It has become commonplace to see headlines such as 'Scotland eyes Singapore in "radical” overhaul of teaching career paths' (Hepburn, 2017) or 'Highly trained, respected and free: why Finland's teachers are different' (Crouch, 2015).

Yet, as these headlines suggest, Finland's and Singapore's respective teaching professions are lauded for different traits. While international commentary often highlights Singapore's teaching career progression that creates incentives for teachers to incrementally develop their practice (e.g. Nelson, 2016), discussions about Finland frequently focus on the professional autonomy that its teachers enjoy (e.g. Crouch, 2015). These differences derive from disparate approaches to teacher accountability. Singapore has an extensive teacher performance management system incorporating detailed performance standards, regular formal appraisals, a career ladder and competition-based salary bonuses (Kan, 2014; Sclafani and Lim, 2008). In contrast, the teaching profession in Finland has minimal rewards and penalties, with neither formal evaluation nor promotion (Finnish National Board of Education, 2013; Sahlberg, 2015).

Strikingly, these disparate approaches to teacher accountability both appear to be effective. Notwithstanding Singapore's recent dominance of TIMSS and PISA league tables and Finland's recent declines in the same (Martin et al., 2016a,b; OECD, 2016), both the education systems continue to receive international adulation, and justifiably so. What accounts for these different pathways to comparable educational success? 
I propose that the answer lies in how teacher accountability instruments affect teacher motivation, and how this accountability-motivation link is shaped by sociocultural patterns in the macrosystem. To preview the argument, one crucial macrosystemic pattern is that both the countries enjoy high public trust in the education system - but this trust is distributed differently across the two systems. In Finland, public trust permeates every level of the education system. Accordingly, at the classroom level, teachers are trusted to work autonomously, with little formal monitoring. However, in Singapore, public trust is focused upon the Ministry of Education and its capacity to deliver good learning outcomes system-wide - hence the extensive, centrally steered system for managing the teachers' performance. Despite these differences, both the systems experience a similar feedback loop between trust, accountability and teacher motivation: the distribution of trust legitimates the choice of teacher accountability instruments,

\section{BOX 1: INTERVIEW METHODS}

In this chapter, I draw on interviews with 12 teachers from 11 different secondary schools in Singapore (for pupils from ages 13 to 16/17), and 12 teachers from 10 lower secondary schools in Finland (for ages 13-15). The interviews were conducted during July 2018 and September 2018, respectively. These interviews are part of a larger mixed-methods research project on teacher accountability policy and sociocultural context across countries, which also include statistical analysis of sociocultural surveys and international largescale assessments. Accordingly, I interviewed teachers in the level of schooling that corresponded with PISA and TIMSS (Grade 8) participation.

The sample of participants was not chosen systematically, since I relied on personal and professional networks for participant recruitment. However, I ensured that participants varied in the subjects they taught, their teaching experience, administrative roles and school types, and also in personal characteristics such as gender, ethnolinguistic background and whether they grew up in the country or abroad. Each teacher participated in one individual interview, which was audio recorded and subsequently transcribed. I then coded the transcripts using a coding scheme that was based on the theoretical framework and research questions, with revisions and additions to accommodate themes arising in the corpus. Portions of the coded data were summarised into matrices, to give a visual overview of the corpus without the misleading appearance of precision that would accompany percentages or other numerical summaries. Finally, I reread each transcript in full to ensure that the matrices and illustrative quotes accurately represented each interview. Participants are identified by pseudonyms, and interview quotes have been edited for readability. For further details about interview methods, see Hwa (2019). 
which means that teachers respond to the instruments positively, which, in turn, contributes to desirable student outcomes that reinforce the distribution of trust.

\section{Teacher accountability in Finland and Singapore}

If we think of teacher accountability instruments as structures or practices that (1) set standards, (2) convey information and (3) allocate rewards and penalties for teacher practice, ${ }^{1}$ Finland and Singapore differ in all the three categories. In Singapore, all teachers are subject to the education ministry's Enhanced Performance Management System (EPMS), which codifies extensive standards for teacher practice, collects detailed information on teachers' work and administers a complicated system of rewards and penalties for teacher performance. In the words of Mark, an interview participant from Singapore:

I really do think that the EPMS works well for the identification of teachers who are in need of support, and the identification of teachers who are in need of greater opportunity in order to stretch them for greater impact. I also think that, on a simple day-to-day level, [...] it helps to guide the work of every general education officer and senior education officer in the system. [...] Now, from an incentives perspective, it certainly incentivises hard work, if it's done fairly.

In contrast, Finland sets high standards at the point of entry into the teaching profession, through highly selective admissions to teacher training and extensive socialisation during this training. But once teachers are in classrooms, their school leaders, municipal officials and central government authorities generally assume that they are doing their jobs well, unless complaints from students or parents indicate otherwise. According to Finnish interview participant Helena,

You don't need as many control tools when the assumption is that we teachers are professionals who know their job and have the skills, and that we are all interested in the same goals and in delivering the curriculum.

In the rest of this section, I explore these contrasts between Finland's and Singapore's teacher accountability approaches in more detail.

\section{Setting standards for teacher practice}

Finland's teacher accountability system operates primarily by setting high standards for teacher practice. Almost every interview participant named the national curriculum as an accountability instrument. Even if some teachers may not always comply with curricular minutiae, they still value its standard-setting role. For example, while calling the current curriculum 'very complicated to understand' 
and 'sometimes unclear', and saying that there were probably some curricular expectations that she was not fulfilling, Finnish participant Liisa also said:

Well, we need to have a national curriculum. It's definitely a 'must'. If we didn't have it, then we would not have a common ground for the students to continue on to upper secondary school.

In addition to agreeing that the curriculum was important, participants also agreed that it left them substantial freedom in their practice. In Anneli's words, 'The national curriculum gives us guidelines, but I can still do my work the way that I feel is the best way for me, and for my students.'

However, a strong national curriculum is hardly a unique feature among education systems worldwide. Arguably more important than the curriculum are the standards set by Finland's famously stringent admissions processes for teacher training (Malinen et al., 2012; Muhonen, 2017), as well as its rigorous, master's-level teacher training programmes (Sahlberg, 2015; Tirri, 2014). Admissions standards and pre-service training may not fit some conceptions of teacher accountability instruments, but they undoubtedly play a role in Finnish teacher accountability. Admissions standards ensure that those who are chosen to enter the profession are highly motivated to teach well, while pre-service training ingrains the expertise that orients their motivation towards effective practices. Although a few interview participants mentioned neither the admissions criteria nor the pre-service training, this is probably because such point-of-entry instruments may be taken for granted in teachers' day-to-day work. ${ }^{2}$ However, Antero called the stringent admissions processes 'the most important thing' that the government can do for teaching quality. Likewise, Masa said that pre-service teacher training was 'the most influential thing' in teacher-related policy, far more important 'incentives and disincentives and that kind of stuff'.

Singapore's teacher training programme is similarly selective, with one account stating that it admits roughly $12.5 \%$ of applicants (Butrymowicz, 2014), comparable to Finland's 11\% admission rate for class teacher education programmes in 2016 (Paronen and Lappi, 2018). However, Singaporean interview participants did not identify teacher training as an instrument for teacher accountability. The closest they came was Timothy mentioning that he had to apply twice before successfully getting a teacher training scholarship, and Andy saying that one of his lecturers had told their class of trainees that 'sometimes you do need to know when to blow your own trumpet' in order to get a good performance ranking.

Such ranking takes places through the EPMS. The EPMS is the education ministry's version of the national civil service appraisal system, which was itself based on the performance management system used by the Shell Petroleum Company in the 1980s (Ho and Koh, 2018; Liew, 2012; Neo and Chen, 2007; Quah, 2010). Central to the EMPS is its teaching career ladder, which has three 
tracks: teaching, leadership and senior specialist (Crehan, 2016b). The system sets performance standards in several key results areas under three outcome categories, which vary according to the teacher's position on the career ladder as well as their career track (Kan, 2014). To illustrate, Joseph said that:

Under the teaching track, there are three areas that they will look at. The first, nurturing the child, will encompass your subject and your form teacher responsibilities, as well as aspects of your CCA [i.e. cocurricular activity]. Then you have professional development—so, what are the training plans that you have? Another one is organisational contribution-so, what are the portfolios and school programmes that you actually contribute to?

This comprehensive, tiered system of standards contrasts with the approach described by Finnish interview participants, who emphasised the pedagogical freedom afforded by the curriculum. Besides EMPS structures, many interview participants also mentioned accountability instruments within their subject departments. Department-level instruments include standards for syllabus coverage and assessment frequency - and also for student achievement in the national standardised exams that loom large over the school system.

\section{Collecting and conveying information on teacher practice}

While test scores are pivotal to many teacher accountability systems, pupils in Finland do not take any national standardised tests until they reach the matriculation exam for university entry. Instead, in many schools, one source of information on teacher practice is an annual developmental discussion between each teacher and their principal (Kumpulainen and Lankinen, 2016). While every interview participant mentioned such discussions, some said that the discussions did not take place every year, or that they took place in groups of subject teachers rather than as targeted individual reviews. ${ }^{3}$ Most said that the discussions were informal in tone, involved two-way feedback and did not lead to any follow-up. In Maarit's account:

I think that the sitting down and talking are sometimes forgotten after that. I'll write down something fine, and then we talk, and then the year, every day continues, and probably now I don't even remember what I answered.

Apart from the developmental discussions, sources of information about teacher practice that were identified by interview participants include self-initiated collaborations between colleagues (e.g. to develop content for a particular unit, or to remedy a classroom problem), and discussions or lesson observations triggered by parental complaints. Such parent-triggered interventions appear to 
be rare, with participants mentioning only a few specific incidents over their careers. (That said, a few participants did mention that parents have become more demanding in recent years, especially in more socioeconomically privileged areas.)

Besides these relatively sparse instruments that collect and communicate information in order to directly influence teacher practice, there are some monitoring instruments that collect information on teacher practice in order to facilitate administrative decisions. The Finnish Education Evaluation Centre administers a system of sample-based tests to monitor national educational quality (Vainikainen et al., 2017). However, these sample-based tests do not play an accountability function in individual teachers' work. In Kristiina's words, 'I have never known anybody, or any school, or any teacher who has taken part of them. So they are not related to the everyday work of a teacher.' Similarly, Satu mentioned that her municipality collects feedback about some aspects of teacher practice and job satisfaction but added that:

It's not a very important thing for me when I'm working from Monday to Friday. I don't think it about it very much. [...] It's just a way to transfer the results to higher levels, but it doesn't come down to me again. [...] The municipality gives the money and the guidelines about what work you have to do. So they have to follow up about whether everything is okay, and if there are things to do better, in the future. It's important. But most of the teachers continue teaching the same way, and it doesn't affect them so much.

Each of Finland's municipalities - of which, in 2018, there were 311 (Statistics Finland, 2018) - has considerable decision-making power over local education (European Commission/EACEA/Eurydice, 2018b; Simola et al., 2009). As a result, some municipalities frequently collect information from teachers (Emilia: 'a massive amount of different questionnaires'), whereas others do not (Hannele: 'not at all').

On the other hand, under Singapore's centralised EPMS structures, all teachers regularly report on their work through formal channels that inform annual performance grades. These performance grades are awarded on the basis of several sources of information. At the beginning, middle and end of each school year, every teacher is required to document their targets and achievements on an EPMS form, and then to discuss their performance in a work review session with their reporting officer, i.e. a teacher with management-level responsibilities. Teachers are also observed in the classroom once a year by their respective reporting officers, who also examine a sample of students' work. These sources of information are then discussed at appraisal panels, where reporting officers triangulate each other's observations and compare teachers across each level of the career ladder, before allocating performance grades for the year. This can lead to 
the sense that teachers are constantly under observation by all reporting officers in the school. As Sonia said:

Every time someone walks by, you know you are being judged. Say you turn up five minutes late to the parade ground for assembly, you know that someone out there is eyeballing you and marking you down and saying, 'Okay, this is the person with the so-called punctuality problem.'

Besides heightening self-consciousness and stress among some teachers, the informational requirements of the EPMS generate substantial administrative work for reporting officers, who may have to appraise numerous colleagues (Eleanor: 'sometimes you have got a good ten staff to oversee, plus all the other admin work').

Although EPMS criteria do not officially include student test results, almost all participants mentioned accountability pressures from tests and exams, whether national exam results at the end of secondary school or school-level tests and exams throughout the year. Another frequently cited teacher accountability instrument was parental feedback. Participants gave varying accounts of the frequency and intensity of parental feedback, but it was clear that this feedback could sometimes be onerous. For instance, Mark said that 'parents expect the teachers to be on call 24/7 for student needs', and Maggie noted that some teachers use phone number masking services in order to forestall such $24 / 7$ contact.

Interestingly, Finnish and Singaporean participants had similarly mixed views on the extent to which professional collaborations with other teachers functioned as an accountability instrument. Among Finnish participants, some spoke of regular collaborations, especially with colleagues who teach the same subjects. However, others said that collaboration was infrequent or only happened in specific situations, such as adapting new curricular requirements to their school's needs. A few noted that levels of collaboration vary from school to school. Partly because of these differing levels of collaboration, participants also mentioned different degrees of accountability from colleagues, ranging from clear accountability relationships (e.g. Emilia: 'At least in this school, because we collaborate so much, it's more difficult to do things your own way, or to cut corners, or things like that') to tenuous ones (e.g. Satu: 'Nobody knows what I do in my classroom, [...] only myself and the students, but not my colleagues or the headmaster, nobody'). Likewise, some Singaporean participants did not mention peer collaboration at all, whereas some said that they collaborated regularly with colleagues, whether through formal departmental structures and professional learning communities or via informal information-sharing and self-initiated partnerships. One participant, Maggie, said that most Singaporean teachers 'hoard their materials and information quite a lot' - but also described an intensive, yearlong collaboration with a colleague as her proudest achievement in teaching. These similarly mixed pictures of professional collaboration among teachers contradict 
both the image of Finland as a haven of teacher professional collaboration (e.g. Strauss and Sahlberg, 2015), as well as the image of Singaporeans as relentlessly competitive, selfish people (e.g. Pierson, 2019).

\section{Allocating rewards and penalties}

Finland's teacher accountability instruments for allocating rewards and penalties are similar to its instruments for collecting information on teacher practice: they are usually unobtrusive, and they vary across municipalities. Rewards come in the form of small salary supplements for teachers who take on extra tasks that are otherwise uncompensated - rather than bonuses based on how well a teacher has performed their tasks. Some of these supplements are part of the union-negotiated salary structure, while others come from the municipality's discretionary budget. Supplements from the discretionary budget are allocated in different ways (e.g. Antero: based on the principal's decision; Liisa: based on an application to the municipality) and for different tasks, whether routine (e.g. Kristiina: 'if you take care of the annual choir performances at the school') or ad hoc (e.g. Emilia: 'a project which has touched the whole school'; Juhani: 'when there's been renovations in some schools, and you had to figure out new ways of teaching and change places a lot').

Although there may be some element of merit in awarding the supplements for ad hoc tasks or in how the routine tasks are allocated, most participants did not regard these supplements as merit-based. (In Masa's words: 'I see that as, "If you want to do this crap job, then we'll give you money for it." And some crazy person is going to be okay with that, whereas the rest are like, "Phew, thank goodness I don't have to do it."') Different municipalities distribute these salary supplements differently, and they can be so inconspicuous that two interview participants from the same school disagreed about whether or not these supplements existed. Where they do exist, participants regarded them as token sums (e.g. Liisa: 'around a hundred euros a month'; Emilia: 'a gift card somewhere for fifty euros or something'; Satu: 'the [salary] difference is very low, maybe you can buy one movie ticket'). As for penalties, besides the developmental actions that can be triggered by parental complaints, the only penalty that participants identified was the possibility of being fired for egregious misconduct, such as drunkenness or physical violence in school.

In contrast, every teacher in Singapore is eligible for an annual performance bonus - or subject to career progression penalties - based on their performance grade. According to Mark, top-performing teachers in Singapore can receive annual bonuses of up to 3.5 months' salary. Performance grades also affect the speed at which teachers are promoted through the career ladder. Additionally, good performance grades render teachers eligible for funded study leave (as noted by Maggie), whereas unsatisfactory grades lead to developmental coaching and extra monitoring (as noted by Jane and Joseph) and, eventually, firing (as noted by Maggie and Mark; see also Singapore Teachers' Union, 2014, 2015). 
A noteworthy feature of the EPMS is that performance grades do not derive solely from EPMS standards. Rather, as Geok Ling observed, 'It's criterionreferenced, and then it's also norm-referenced'. Within each school, performance grades are awarded on a forced curve that benchmarks teachers against colleagues of the same level of the career ladder. Official guidelines about the EPMS grading system are not publicly available, but one non-Ministry source says that approximately $30 \%$ of teachers receive $\mathrm{A}$ or $\mathrm{B}$ grades, $65 \%$ receive $\mathrm{C}$ grades and $5 \%$ receive D or E grades (McMillan, 2017). Most participants mentioned that the competitive grading system can generate stress and demotivation for some teachers, and that a small minority of teachers attempted to game the system (e.g. by shifting time away from classroom preparation towards more visible committee work). However, despite its inbuilt competitiveness, and despite participants' acknowledgement that the fairness of the grading system could vary vastly across schools and reporting officers, the EPMS did not appear to undermine collegiality among teachers. In Andy's words, 'We do recognise those who are deserving of credit because [...] something about them enables them to go above and beyond for the students, and we don't begrudge them if they are rewarded accordingly'.

\section{Compatibility among teacher accountability instruments}

In Finland, selection processes for admission into teacher training ensure that those who enter the profession are intrinsically motivated to do their jobs well, and carefully designed pre-service teacher training programmes ensure that teachers know what such effectiveness looks like. This lessens the need for standards, informational channels and reward systems for controlling the in-service processes and outputs of teachers' work. In this setting, such external controls are not only unnecessary, but also undesirable. As Deci and Ryan (2000) famously argue, extrinsic rewards and other forms of control can impinge on the sense of autonomy that is crucial to sustaining intrinsic motivation (see also Chapter 2 of this volume). Furthermore, several participants emphasised the need for teachers to have the freedom to use their skills and personalities to facilitate student learning as they see fit. Such autonomy would be incompatible with exhaustive standard-setting and information-gathering instruments.

Autonomy notwithstanding, the input standards are strong enough to lessen the risk that teachers will deviate from the overarching goals of the education system. The government's teacher accountability instruments may be concentrated at the point of entry into the profession, but these standards are sustained - and perhaps reinforced - over time. According to Päivi, an experienced teacher who had once been in school leadership before opting to return to the classroom:

When I was the headmaster, I had the idea that when young teachers come to the school, then we could learn so many new things from them. 
[...] But then I saw that, after a year or two, new teachers are just the same as old teachers. [...] But I wouldn't change it. Teachers are kind and they are caring and they are hoping for the children's best. And that's the way it is.

Thus, the rigorous input standards offset the risks generated by the sparseness of formal teacher accountability instruments for processes and outputs. The capacity of these standards for reducing deviation is also evident in the fact that Finland has consistently had the smallest between-school variance in PISA scores among OECD countries (with the exception of PISA 2003, when Iceland had a marginally lower variance, and PISA 2000, when Sweden and Iceland had lower variances; OECD, 2004, 2007, 2010, 2013, 2016; OECD and UNESCO Institute for Statistics, 2003).

In Singapore, the teacher accountability system hinges on extensive information and powerful extrinsic incentives. This means that it would be vulnerable to failure if either the information or the consequences proved inadequate. But this vulnerability is mitigated by the magnitude of the performance bonuses, which can influence teacher motivation considerably. (In Adeline's words: 'Singaporean teachers are very typical civil servants, and they like to have their various KPIs and know that if they meet them, they might get rewarded'.) Furthermore, EPMS job descriptions for management-level positions in the career ladder ensure that enough time is channelled towards collecting information on teacher practice. Peter, who had recently become a subject head, said the following about his promotion:

It has changed my outlook of who I am as a teacher. [...] Because I know that my performance is going to be evaluated in the lens of being a subject head, I guess that shifts my focus a little when it comes to how much I'm involved in things that aren't subject-related.

Thus, there is compatibility between the standard-setting, informational, and consequential elements of the EPMS. The standards designate resources for information collection (among other things), while information enables the allocation of consequences, which, in turn, shore up the influence of the standards.

Collectively, these EPMS instruments exert extensive government control over teachers' work. However, this impingement on teacher autonomy is somewhat mitigated because government expectations of teachers often converge with what teachers expect of themselves. In particular, several teachers mentioned that they appreciated the ministry's emphasis on both academic and socioemotional development. When asked whether ministry expectations overlapped with her own, Sonia said:

I think they do converge quite a bit. [...] Why I got into [teaching] was because I want to help mould the character of students, and it does give me a lot of satisfaction when I see my students perform well academically. 
Hence, good policy design softens teachers' experiences of extensive accountability instruments. While criticising some aspects of the EPMS, Timothy also said he was 'intrigued and amazed' at the comprehensiveness of its rubrics. Similarly, Maggie said that the ministry's pedagogical recommendations 'are not really giving you trouble; they actually make your life simpler'. Again, international assessment results offer further support: in 2015, Singapore topped the tables across all PISA and TIMSS subjects and levels (Martin et al., 2016a,b; OECD, 2016).

\section{Summary}

As shown in Table 11.1, Singapore's teacher accountability system is far more extensive than Finland's in almost every respect. The sole exception is standard-setting at the point of entry to the teaching profession, which plays a bigger role in Finland than in Singapore. Besides these entry standards and the national curriculum, Finland's instruments for communicating information and allocating consequences for teacher practice are comparatively low-key and can vary considerably across schools and municipalities. In contrast, Singapore's EPMS structures for teacher accountability are highly centralised and influential. The pervasiveness of this performance management system is evident in the fact that every single Singaporean interview participant, when asked about teacher accountability instruments, mentioned EPMS key results areas, performance reporting forms, thrice-yearly work review sessions, performance grades and promotions.

\section{Different teacher accountability approaches, comparable efficacy?}

Given these stark differences between Finland's and Singapore's approaches to teacher accountability, how can we understand the high quality of teaching in both of these countries? One way to interpret this is that teacher accountability

TABLE 11.1 Summary of teacher accountability instruments in Finland and Singapore

\begin{tabular}{lll}
\hline & Finland & Singapore \\
\hline Setting standards & $\begin{array}{l}\text { National curriculum; strong } \\
\text { standards at the point of entry } \\
\text { to the teaching profession }\end{array}$ & $\begin{array}{l}\text { Extensive teacher performance } \\
\text { standards in the EPMS }\end{array}$ \\
$\begin{array}{l}\text { Communicating } \\
\text { information }\end{array}$ & $\begin{array}{l}\text { Annual developmental } \\
\text { discussions with the principal } \\
\text { in most schools }\end{array}$ & $\begin{array}{l}\text { Annual lesson observations; thrice- } \\
\text { yearly performance management } \\
\text { reports and discussions with } \\
\text { reporting officers }\end{array}$ \\
$\begin{array}{lll}\text { Allocating } \\
\text { consequences }\end{array}$ & $\begin{array}{l}\text { Small salary supplements for } \\
\text { additional responsibilities; firing } \\
\text { for egregious behaviour }\end{array}$ & $\begin{array}{l}\text { Performance-based annual bonuses } \\
\text { and career progression }\end{array}$ \\
\hline
\end{tabular}


instruments are irrelevant to the quality of teachers' work and do not make any positive contribution to teacher practice. A second interpretation is that teacher accountability instruments can, in fact, contribute to teacher practice: disparate approaches to teacher accountability can be equally effective, as long as the instruments are well designed. A third possible interpretation agrees with the second in arguing that accountability instruments do affect teachers' work, but diverges from the second in further asserting accountability instruments have different effects depending on how well they fit the larger contexts in which they are embedded.

Based on the interviews I conducted as well as secondary sources, I believe that the third interpretation is the most plausible. Teacher accountability instruments can, indeed, influence teacher practice constructively - but only if the instruments are compatible with the macro and exo-contexts in which they are implemented. To give some suggestive evidence, Figure 11.1 summarises interview participants' responses when I asked them what would happen, hypothetically, if their country adopted the other country's approach to teacher accountability (i.e. if Finland introduced EPMS structures, or if Singapore removed the career ladder, formal teacher appraisal and performance bonuses). Note that I asked this question in an open-ended manner, so the statements in Figure 11.1 represent my summaries of participants' responses, rather than a set of statements presented to participants for their agreement or disagreement.

Regardless of their personal preferences, none of the participants believed that the other country's teacher accountability approach would definitely be preferred by most teachers and/or would improve education in their country. Several Singaporean participants said that they would personally prefer the non-competitive autonomy of Finland's approach - but even these participants doubted that it would work at the system level, because they anticipated that some teachers would dislike the relative lack of structure and the absence of performance-based reward. For example, Singaporean participant Maggie said that the Finnish approach would give her the flexibility to develop her teaching practice long term rather than focusing on short-term performance targets, but added that:

- agree partially agree $O$ disagree [ ] not mentioned during the interview

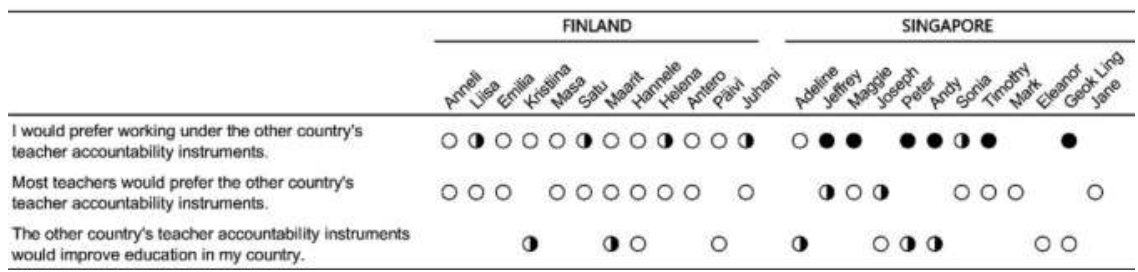

FIGURE 11.1 An summary of interview participants' answers when asked what would happen if, hypothetically, their country adopted the other country's teacher accountability instruments 
I think if you suddenly changed the system, most of the teachers will be very stressed because they have no idea what is being observed. Singaporeans find comfort, I think, in knowing exactly what is expected, and they like to do it to the letter.

Similarly, Finnish participant Juhani appreciated that Singapore's EPMS recognises good work and gives teachers a structured way of identifying their strengths and weaknesses but said that:

We are so independent here. And we like that independence in our classrooms so much, that even that bonus would not make this system a good thing. [...] And we are so equal, among teachers. [...] We do not want to give others the possibility of rushing higher. [...] We have done some studies about rewarding people with money, and it gives satisfaction for shorter period of time than when you are valued by the society you work in.

Given these responses, it seems unlikely that teacher accountability instruments have no influence on teachers' work, as in the first possible interpretation. And given how strongly participants reacted against the other country's accountability approach (e.g. Masa, describing Finnish teachers: 'They would quit. They would go on strike'; Sonia: 'The average Singaporean teacher will probably be up in arms'), it seems unlikely that any well-aligned set of accountability instruments could be equally effective in any given setting, as in the second possible interpretation. Internal coherence within a set of teacher accountability instruments may be necessary for efficacy, but it is not sufficient. ${ }^{4}$ Instead, as in the third interpretation, teacher accountability instruments need to be compatible with their respective macrosystems in order to be effective.

\section{Teacher motivation as the key to teacher accountability}

The key to making sense of Finland's and Singapore's disparate but comparably effective approaches to teacher accountability lies in (1) the relationship between accountability and motivation, and (2) the relationship between motivation and context. First, motivation - defined by Schunk et al. (2010, p. 4) as 'the process whereby goal-directed activity is instigated and sustained' - is closely connected to accountability. Whether implicitly or explicitly, accountability instruments targeting teacher practice assume that student outcomes can improve when teachers work harder, i.e. with raised motivation, and/or work differently, i.e. with motivation redirected towards different goals (see also Kozlowski and Lauen, 2019). Thus, teacher motivation is the linchpin of accountability instruments that successfully change teacher practice.

All three accountability mechanisms discussed in the previous section - setting standards, communicating information and allocating consequences - can influence teacher motivation, although they do so in different ways. For example, 
informational instruments influence teachers' motivation via their desire to be regarded favourably when information about their practice is compared to stakeholder expectations. When asked how accountability instruments affected his work, Timothy, a Singaporean interview participant, responded:

It's always at the back of one's mind. [...] Yeah, because you know that there are other people who are watching you, so to speak. [...] I would consider it a negative motivation, or not the most desired form of motivation. But at least it helps to spur you on when you are drained.

Informational instruments can vary greatly. There are many differences between, for example, a mandatory questionnaire on classroom activities and a self-initiated troubleshooting session with colleagues. Despite these differences, any information-based accountability instrument that successfully shapes teacher motivation does so by prompting teachers' desires to compare favourably to a set of expectations, whether codified or tacit, shared or unilateral, precise or vague, externally imposed or personally espoused.

These expectations may come from accountability instruments that set standards for teacher practice. Unlike the informational mechanism, which operates through teachers' awareness that they are being actively compared to a set of expectations, the standard-setting mechanism passively directs teacher motivation towards such expectations. Passivity notwithstanding, standards can be highly influential. For example, Satu said the following about Finland's national curriculum:

I have the basic rules there. So it's easier than thinking, 'Whoa, what I will do now?' The year is not empty. [...] And it's easier for the teacher to follow the curriculum than invent something by themselves.

As with informational instruments, standards can take on a variety of tones in a range of relational settings. Standards can safeguard community networks through shared norms, or channel competition towards remunerative targets.

Finally, and perhaps most obviously, some accountability instruments allocate consequences based on stakeholders' judgements of teacher practice - thus influencing teacher motivation via the desire to gain rewards and avoid penalties. When asked about the consequences of teacher performance grades, Geok Ling, a Singaporean teacher in middle management, said:

You know that, 'Okay, they're going to grade me like this, and this is how I can get more because of the bonus.' So people will drive their behaviour towards the criteria. [...] But you can argue that it's a necessary devil; it's a double-edged sword. While it may raise the standard and it motivates some, because they see it as an affirmation, it will also demoralise some. 
As Geok Ling observes, teachers' perspectives on accountability instruments can be heterogeneous, yielding heterogeneous effects on motivation.

To illustrate the degree to which accountability instruments may potentially change teacher motivation, consider interview participant Masa's description of Finland's new Kilpailukykysopimus (KIKY, i.e. competitiveness pact) policy. KIKY stipulates, among other things, that all full-time employees nationwide work an extra 24 hours annually without extra pay (SAK, 2016). This emphasis on regulating work hours is a clear departure from Finland's established approach to teacher accountability, in which careful selection processes and pre-service training construct a competent and motivated body of teachers who experience relatively few external controls over their day-to-day work. In Masa's view, the mismatch between KIKY stipulations and this established approach was damaging:

Teachers started complaining about being a teacher, which was new, for me. People were starting to think about changing jobs. Because all of a sudden, we've got this bureaucracy that's keeping track of this time. They're realising that we already do more than that anyway. And [...] now they're doing it for the time, which equates to money, rather than because something inside says, 'This is what needs to be done.' So the teachers' personal standards have dropped.

Although the new cross-sector KIKY policy may have eroded some intrinsic motivation, most Finnish interview participants emphasised the long-established internal standards that Masa also mentioned. They agreed that most Finnish teachers are highly motivated to teach well, despite the negligible rewards and penalties.

\section{How the macrosystem shapes teacher motivation and teacher accountability}

So, what matters in teacher accountability is not whether the accountability instruments are extensive (as in Singapore) or less extensive (as in Finland). Rather, what matters is that the instruments influence teacher motivation in ways that lead to desirable student outcomes. Like other professionals who serve multiple goals that are difficult to monitor and measure, teachers constantly and actively redistribute limited resources between numerous priorities (Lipsky, 2010; Murnane and Cohen, 1986). And they do not respond mechanistically to accountability instruments. Satu, who was quoted earlier speaking favourably about Finland's national curriculum, also said that:

Even if they make quite a big change to the curriculum, some teachers [...] may say, 'Huh, pfft. Waste of time. I'll do what I have done for the past thirty-five years.' 
Similarly, Maggie, a teacher from Singapore, observed that:

Let's say you have a top-down policy implementation. If you didn't trust [the teachers] with the discussion beforehand, then when you do implement it, they would just do whatever it takes to survive, and it might not turn out the way you want it. Like, they will modify it, just to placate you and show you some semblance of what you want to see.

Satu's and Maggie's remarks illustrate an argument advanced by Andrews et al. (2017, pp. 113-118) that effective public service delivery relies not only on the administrative apparatus of accountability but also on the alignment between each actor's duties and their sense of personal and social responsibility (see also McLaughlin, 1987).

Simply put, an accountability instrument will only influence a teacher's motivation positively if the teacher regards the instrument as sufficiently meaningful, legitimate or otherwise persuasive (Verger and Parcerisa, 2017). In their review of psychological research on how accountability affects social choices, Lerner and Tetlock (1999) found that accountability only leads to beneficial increases in cognitive effort when numerous contingent factors coincide. One such factor was whether the accountability instrument was seen as legitimate. Similarly, in an analysis of an accountability policy change in a US state, Kim et al. (2019) matched data from teacher self-report questionnaires with principal-reported evaluation ratings and found that teachers who viewed the new evaluation policies as legitimate were more likely to improve their instructional practice.

There is a close connection here between legitimacy and trust. As noted in Chapter 1 of this volume, trust depends on the perception that the recipient of the trust is competent, benevolent and has integrity. These three traits clearly (though not completely) overlap with the three traits that Kim et al. (2019) identify as key ingredients in regarding a policy as legitimate: whether its instruments are valid and reliable, which overlaps with competence; whether it is procedurally fair, which overlaps with integrity; and whether its intended outcomes are worthwhile, which is somewhat analogous to benevolence (see also Tyler, 2006). Hence, if a teacher believes that the design and intent of a teacher accountability instrument do reflect these traits, they are more likely to regard the instrument as legitimate.

However, as noted earlier, teachers face competing priorities in their daily work, and even a legitimate accountability instrument may not influence teacher motivation if it is superseded by other priorities, as in Satu's observations on how some Finnish teachers' respond to curricular change. Conversely, even if a teacher does not regard an instrument as trustworthy, it may still influence their motivation, as with Finland's KIKY stipulation that Masa described. Furthermore, as Singaporean participant Geok Ling observed, a legitimate accountability instrument can have heterogeneous effects, raising the motivation of some teachers while demotivating others. 
Heterogeneity notwithstanding, there will be some broad patterns in the accountability instruments that most teachers within a given setting regard as compelling. This is because teachers' perspectives and, in turn, the overall efficacy of teacher accountability instruments are shaped by context. As Pawson and Tilley (1997, p. 216) observe in their seminal work on realist policy evaluation, 'subjects will only act upon the resources and choices offered by a program if they are in conducive settings'. Similarly, Bronfenbrenner (1977, p. 515) observes that macrosystems 'set the pattern for the structures and activities occurring at the concrete level'. These macrosystems comprise societal 'blueprints', or 'the overarching institutional patterns of the culture or subculture' (Bronfenbrenner, 1977). Hence, the interview participants' vehement reactions against the other country's accountability approach: Finland's macrosystem is not a conducive setting for Singapore's EPMS, nor is Singapore's macrosystem a conducive setting for Finland's light-touch approach.

\section{Compatibility between teacher accountability instruments and macro-level trust}

How does this play out in Finland and Singapore? First, as shown earlier, each country's set of teacher accountability instruments is well designed, with compatibility between the instruments such that they collectively influence teacher motivation in desirable ways. Finland's teacher accountability instruments establish and orient high levels of teacher motivation at the point of entry into the teaching profession, while Singapore manages and motivates teachers' work throughout their careers through a system of interlocking standards, monitoring structures and performance-based consequences. But internal compatibility is not enough. Another factor in their successful approaches to teacher accountability is compatibility between their accountability instruments and the wider macrosystem. In the absence of such external compatibility, the accountability instruments would fail to influence teacher motivation as intended by policymakers, instead of having negative or null effects - as with Finland's KIKY regulations that may have compromised teachers' intrinsic motivation.

Teacher accountability is influenced by numerous interacting macrosystemic patterns. I focus here on one such pattern: the distribution of public trust in the education system. ${ }^{5}$ Whereas the absence of external compatibility between teacher accountability instruments and the macrosystem can yield negative or null effects, the presence of such external compatibility may result in a positive feedback loop. If the choice of teacher accountability instruments is aligned with the distribution of trust, this trust will legitimate the instruments, thus increasing their likelihood of raising or positively redirecting teacher motivation. These positive motivational changes may then improve classroom practice and student outcomes, which, in turn, will shore up trust in the education system. 
Finns and Singaporeans both place great trust in their education systems, but the distribution of this trust differs. In Finland, the trust is spread throughout every level of the education system, such that a great deal of trust is invested in teachers as highly trained and motivated professionals. In Singapore, however, the main locus of trust is the Ministry of Education and its capacity to coordinate and incentivise desirable activities throughout the education system. Accordingly, Finland's teacher accountability instruments aim to mobilise and empower teachers to work towards desirable student outcomes, whereas Singapore's teacher accountability instruments pursue the same aim by endowing the government with extensive capacities for shaping teachers' work. Following Cerna's (2014) terminology, the dominant form of trust in Singapore's education system is institutional trust in the Ministry of Education. In Finland, institutional trust in the government is certainly present, but it does not supersede what Cerna calls generalised trust in teachers.

\section{Finland: Generalised trust in teachers alongside standard-setting accountability instruments}

The generalised trust that Finnish teachers enjoy is a form of interpersonal trust. However, while particularised interpersonal trust in a particular teacher is based on first-hand interactions, generalised trust is instead based on a widely shared confidence in teachers' collective professional integrity. In Hannele's words:

I think Finnish people rely on the teachers. I hear people say all the time that, 'I know you have a good school. I know you have good teachers.' [...] They trust that we do our job.

This trust in teachers does not imply a lack of trust in other levels of the education system, but rather that teachers are trusted to have command over their own work. According to Helena:

It goes through the hierarchy. The Ministry of Education trusts the [National Agency for Education] to develop the curriculum based on certain things they've laid out. [...] And then they trust the municipalities to come up with the local curricula. And those municipalities trust the schools to come up with school-based curricula. And then the school administration trusts that the teachers will do something useful with the school curricula in the way that they deem best, using the books, materials, and methods they find best. And then I, as a teacher, trust that when I tell my students to do something, they will do it.

Thus, actors at each level of educational delivery are trusted to do what is expected in their respective spheres. 
In the sphere of the classroom, both the general public and the government trust that teachers will do their best. Consequently, this generalised trust in Finland's selected and highly trained teachers legitimates the relative lack of formal teacher accountability. In Antero's words:

When they selected me to study teaching, of course they checked that my personality and who I am fits the job. And after that, I have been on my own. Nobody has come here to say that, 'You must change. And you must do it like this, not like that.' I am in charge here.

Concurrently, the successful track record of this approach reinforces public trust in teachers. According to Liisa:

There haven't been any discussions about Finland adopting any teacher accountability instruments from other countries. I think there's a consensus that the thing runs well now, so there are no very big problems about teachers not doing what they're supposed to do. [...] We know what we need to do. So we don't need an extra system to tell us what to do in that way.

Hence, the highly motivated and trained people who succeed in entering the teaching profession enjoy a virtuous cycle of autonomy and public trust (see also Aho et al., 2006; Crehan, 2016a; Simola et al., 2017). As early as 1981, a Finnish government official spoke to an OECD delegation about 'the importance of traditional freedom for teachers in Finland' to choose their teaching methods, textbooks and curricular emphases (OECD, 1982). Despite this longstanding autonomy, Finnish teachers have not always been so highly esteemed. Simola (2014) observes that, prior to the 2000s, most Finns regarded the school system as good, though not exceptional, but some influential business leaders were criticising the system vociferously and advocating for more educational competition - until the 2001 release of PISA results silenced them. Thus, PISA league tables inadvertently served as an accountability instrument, conveying the information that Finland's teachers were collectively doing a good job, thus easing the national-level pressure to apply more accountability instruments to teachers.

Beyond education, an oft-cited indicator of generalised trust is the proportion of people answering affirmatively when asked whether 'most people can be trusted' or 'you need to be very careful in dealing with people'. In the European Values Study (EVS) 2008, Finland had the fourth highest proportion of respondents favouring generalised trust, only exceeded by Nordic neighbours Denmark, Norway and Sweden (EVS, 2016). While Finland and its Nordic counterparts did not participate in the more recent World Values Survey (WVS) 2010-2014, which included the same questionnaire item, only the Netherlands exceeded the proportion of Finnish EVS 2008 respondents favouring trust, and marginally so (WVS Association, 2015). 
This balanced distribution of trust works in tandem with another important macro-level value: a societal emphasis on egalitarianism. Both of these values are evident in Helena's account of how some foreign exchange students at their school reacted to school cafeteria food that seemed tasteless to them:

Their solution to it was very simple: 'Why don't you just tell the kitchen to make spicier food?' Which makes sense from the point of view of, 'The kitchen staff's job is to serve me food,' and, 'They're lower in the hierarchy.' But from our point of view, the kitchen is run by a company, which we buy the service of. And they're just people working in the kitchen. And they don't come and tell me how to teach maths, so I wouldn't feel like it was my job to tell them how to make food.

Finland's egalitarianism is evident in, among other things, its relative economic equality. During the 10 most recent years for which Gini data are available (2005-2015), Finland consistently had 1 of the 10 most equal income distributions in the world (World Bank, 2019). Finland also had the third highest score in Oxfam's 2018 Commitment to Reducing Inequality index (Lawson and Martin, 2018).

\section{Singapore: Institutional trust in the education ministry and extensive instruments for teacher accountability}

On the other hand, Singapore had the eighth lowest score in the same Commitment to Reducing Inequality index (Lawson and Martin, 2018). One interview participant, Jeffrey, suggested that Singapore's tightly packed urban geography facilitates ubiquitous stratification:

You're kind of fenced in. [...] So that very much forces you higher up on the value chain. You basically can't choose to slack off. [...] . I mean, you can say publish or perish-but you don't even have the option (laughter) in the education system. It's pass or distinction. That's it. (laughing) Because no matter how badly you do, they're going to put you somewhere, whether it's ITE [i.e. Institute of Technical Education], Normal (Tech), or Foundation. There's somewhere that they have specially designated for you.

Jeffrey's remarks imply not only socioeconomic stratification (with a 'value chain' and a ladder of educational prestige), but also political hierarchy (with constraints on individual agency, since you are 'fenced in' and 'they', i.e. the government, designate your place in society). The combination of hierarchical governance alongside educational and socioeconomic stratification pervades the public sphere in Singapore, having been fostered by the government under the banner of competitive, progress-oriented meritocracy throughout the second half of the 20th century (Lee, 2000; Tan, 2018; see also Teo, 2018; Tremewan, 
1994). Some interview participants expressed discomfort with this emphasis on achievement, meritocracy and competition - whether in the EPMS rewards system or in Singapore's macrosystem more broadly - but they also assumed that it was ingrained in both local culture and national governance and was unlikely to change.

One element of Singapore's sociopolitical stratification is a hierarchical distribution of trust and authority in the education system. In Singapore, public trust in the education system is not distributed throughout the system, as in Finland. Instead, it is concentrated on the government. In the WVS 2010-2014, Singapore had the fourth highest proportion of respondents - after China, Qatar and Azerbaijan - reporting that they had 'a great deal' or 'quite a lot' of confidence in their central government (WVS Association, 2015).

When I asked interview participants whether their lived experience supported the WVS finding that most Singaporeans were highly confident in public institutions, every participant agreed. (However, some added that younger generations have started to question the government.) The precedence of trust in government over trust in teachers was also evident in some participants' responses to the hypothetical question about Finland's teacher accountability approach. For example, Eleanor said that:

My immediate response is, it's not going to work. [...] It will be very teacher-dependent, and so it will be the luck of the draw if your child gets into this classroom where this teacher is a bit more progressive or has more initiative, then you benefit. Whereas in other classrooms where the teacher is just happy with what she or he is doing, then the child is not going to learn as much. So that inconsistency is something the government will not want to risk, because the feedback from the people will be quite strong. I think there is a great sense of entitlement here, where the people depend a lot on the government to get things done.

Andy's response shows a similar dynamic. Andy described himself as a 'happy ordinary teacher', a moniker adopted by some Singaporean teachers who prioritise classroom teaching over climbing the career ladder towards the ministry headquarters. After I told him about Finland's teacher accountability approach, Andy commented that those who would enter teaching under Finland's approach were likely to be happy ordinary teachers, ${ }^{6}$ and then said:

So if Singapore were to embark on such a system, it's possible that it might be better in terms of attracting the right kind of people. But yeah, where would we train all our bureaucrats? They need to experience life on the ground as well.

This consciousness of system-level needs over teachers' needs was a recurring theme in the Singapore interviews. 
Even though Singapore's distribution of trust differs from Finland's, its teacher accountability instruments are likewise matched to its respective distribution. This yields the same mutual reinforcement: the locus of trust legitimates the chosen instruments, and the efficacy of the instruments strengthens public trust. Specifically, high public trust in Singapore's government legitimates its considerable control over teachers' work. The strength of this trust is such that teachers generally accede to the extensive accountability instruments, despite any personal qualms they may have (see also Ee, 2018). For example, Timothy said:

To use a very civil service phrase, these [EPMS] expectations are all cascaded down from your ministers and the top policymakers. So what they would have in mind, I believe, is in line with what, hopefully, will help make the country a better one.

This affirmation of government authority is especially striking given, less than a minute earlier, Timothy had said that he had 'many reservations' about how the EPMS was carried out. Several participants displayed a similar sense of internal tension when they disagreed with government stances. However, others appeared very comfortable with the hierarchical authority. Furthermore, as Jane suggests, even those who feel internal discomfort may outwardly comply because of the government's success in educational and economic development:

They have confidence [in government institutions] because, all the while, the country has been doing well. But I think, slowly, some of the younger ones are questioning, and some of the older ones want to have some opposition [to the government]. But they don't want to rock the boat, because they're still very happy.

Since the government's extensive control over education and other aspects of public life has proved effective, most Singaporeans continue to trust government institutions. For now, at least, trust in the Singaporean government supports and is supported by - its extensive teacher accountability instruments, whereas public trust in teachers justifies the Finnish government's relatively sparse teacher accountability instruments, and the sustained efficacy of these instruments shores up trust in teachers. ${ }^{7}$

\section{Conclusion}

To summarise, Singapore's education ministry sets extensive standards for multiple areas of teacher practice, and the information collected on these areas informs market-oriented governance of the school system. However, departing from libertarian conventions, teachers and schools are not independent suppliers in a marketplace in which families seek their best options for educational consumption. 
(In fact, the scope of Singapore's educational market is constrained by the stipulation that Singaporean citizens who seek to enrol in private schools require individual authorisation from the ministry [Singapore Ministry of Education, 2018].) Rather, teachers are dependent on the ministry as the sole buyer of their services. Instead of competing for higher enrolments in their schools, teachers compete for larger bonuses and career development opportunities, which are overseen by the ministry. Yet this top-heavy approach to teacher accountability works because of public confidence in the government, alongside established macrosystemic emphases on hierarchy and competition.

In contrast, Finland's government sets strong standards for teacher practice at the point of entry to the teaching profession, but this exercise of hierarchy serves to enable and safeguard subsequent teacher autonomy. Autonomy notwithstanding, teachers in Finland do not conform to an idealised template of network governance. While teachers, school leaders, municipalities and national-level education authorities do work towards common goals as interdependent actors, the dominant logic is not collaborative decision-making, but rather individual responsibility within clearly demarcated domains. Hence, the degree of professional collaboration among teachers may vary from extensive to virtually non-existent. But, for the most part, teachers share a strong work ethic and a commitment to the egalitarian aims of the national curriculum. Thus, hierarchical standard-setting in Finland facilitates loose networks of autonomous teachers, whereas standard-setting in Singapore facilitates centralised quality improvement via professional competition for bonuses, promotions and prestige.

Differences notwithstanding, these successful approaches to teacher accountability share an underlying principle: compatibility between teacher accountability instruments and the macrosystem. Each education system gives the final say in teacher practice to the stakeholders that are most trusted with classroom matters, whether Finland's highly trained teachers or Singapore's highly mobilised education ministry. This compatibility helps to legitimise these instruments in teachers' eyes, which facilitates the influence of the accountability instruments over teacher motivation and teacher practice. In turn, the efficacy of the accountability instruments in facilitating desirable educational outcomes also legitimises public trust in Finland's teachers and Singapore's education ministry. As observed in Chapter 1, the trust often has a reciprocal nature, which can deepen over time.

In aiming for brevity, this discussion has veered into some generalisations. Finland's education system may be premised on egalitarianism, but this does not obliterate the influence of family socioeconomic background on educational pathways. In Helena's words: 'We have research information that students whose parents have academic [rather than vocational] schooling do better academically. [...] So it would be lying to say that doesn't exist'. Also, despite stratification in Singapore, the school system offers a high baseline of education even for the less privileged, given that the lowest socioeconomic quartile of Singaporean 15-year-olds outperformed the average OECD 15-year-old in PISA 2015 
science (OECD, 2016). Moreover, Finland and Singapore may differ greatly, but they are far from being binary opposites. For one thing, both countries share a society-wide affirmation of the value of education, as observed by interview participant Mark. Additionally, interview participants in both the countries expressed a strong sense of intrinsic motivation to teach well, despite the vast differences in the extrinsic incentive structures that circumscribe their work.

Still, the dominant macrosystemic patterns discussed here - about egalitarianism, stratification, autonomy and structure - need to be taken seriously in designing teacher accountability policy: for example, a teacher who grew up in an egalitarian education system may treat a norm-referenced performance bonus very differently from a colleague who was educated in a highly competitive and stratified system. Equally, a teacher who had been socialised to prize their professional autonomy might resent the introduction of detailed guidelines outlining their duties, whereas a teacher accustomed to relying on such guidelines might flounder if they were removed.

In this chapter, I have mapped one pathway through which the cultural macrosystem can have a pivotal influence on individual teacher's responses to exosystem-level policy. However, I am not suggesting that macrosystemic constraints necessarily limit any given education system to a single, culturally dictated model of teacher accountability. Neither I am suggesting that macro-level cultural patterns are the sole - or even the prime - influence on how teachers respond to accountability instruments. Social and professional behaviour is informed by numerous inputs. Even if certain sociocultural patterns are dominant and widely acknowledged in a given macrosystem, these patterns will not be universally shared (Maxwell, 2012). ${ }^{8}$ What I am arguing, in line with the other chapters in this volume, is that teacher accountability takes place in an ecosystem, in which different levels of the system can influence each other multidirectionally. While the cases of Finland and Singapore show how the macrosystem can offer orientations for effective teacher accountability policy, it is equally possible that teacher accountability instruments can shape teachers' macrosystemically situated conceptions of their work (especially when accountability structures are closely aligned with initial teacher training and other aspects of teachers' socialisation, as in Holloway, 2018; Holloway and Brass, 2018). When discussing and designing teacher accountability policy, the macrosystem and its distribution of trust matters - but so do other components of the system.

\section{Acknowledgements}

The research in this chapter was conducted as part of a $\mathrm{PhD}$ funded by the Gates Cambridge Trust (BMGF OPP1144). I am also grateful for fieldwork funding provided by the Faculty of Education and St Catharine's College at the University of Cambridge. 


\section{Notes}

1 Following Bovens' (2007, p. 450) definition of accountability as 'a relationship between an actor and a forum, in which the actor has an obligation to explain and to justify his or her conduct, the forum can pose questions and pass judgement, and the actor may face consequences', I formally define teacher accountability instruments as tools, practices, and structures that aim to orient teacher practice toward stakeholder expectations by (a) collecting information about teachers' individual or collective practice and communicating this information to stakeholders, (b) setting standards by which stakeholders judge teacher practice, and/or (c) allocating consequences based on stakeholders' judgements of teachers' practice.

2 I am grateful to Lucy Crehan for the observation that teacher accountability systems can concentrate their quality controls on different junctures, whether the inputs, processes or outputs of the teaching profession. Similarly, Oates $(2015$, p. 4) observes that the teaching profession in Finland emphasises 'front-end restrictions' rather than 'back-end' ones.

3 For comparison, in a 2017 survey, 65\% of Finnish teachers said they had had one developmental discussion during the prior 12 months, $27 \%$ said they had had two or more discussions, while $8 \%$ said they had not had any (OAJ, 2018, p. 31).

4 Interview participants' generally positive views about their country's respective approaches to teacher accountability do not mean that they were uncritical. In fact, some participants from both countries emphasised that they knew of colleagues who were not especially motivated to teach well, but nonetheless were allowed to remain in the profession. In Finland, this was partly because many teachers hold tenured positions (European Commission/EACEA/Eurydice, 2018a). In Singapore, this room for 'cruising', as some participants called it, could emerge from teachers' seniority, or from favourably misrepresenting one's work to the reporting officer (e.g. showcasing recommended pedagogical strategies only during scheduled lesson observations), or from what one participant called the 'very merciful' lower tiers of the grading system (although other participants disputed these mercies). Besides this room for slack performance, other criticisms raised by multiple participants included the lack of professional feedback on classroom practice in Finland, and the competitiveness, stress, and potential harm to teachers' self-esteem in Singapore. Notwithstanding such shortcomings, the overall picture painted by interview participants was of effective teacher accountability instruments within effective school systems.

5 When conducting these interviews, I was not specifically investigating the distribution of public trust in the education system. I did ask interview participants about some aspects of social capital (including generalised trust) and about how hierarchical/accountability egalitarian their countries were, due to pre-existing studies indicating that these sociocultural constructs may affect the influence of instruments (on social capital, see Iyengar, 2012; Webber, 2010; on hierarchy, see Broekman, 2016; Gelfand et al., 2004;Velayutham and Perera, 2004). However, I only posed these questions about social capital and hierarchy at the end of each interview, after asking more general questions about aspects of sociocultural context that influence education in their country, and that influence teachers' responses to accountability instruments. The centrality of the distribution of trust in the education system emerged from participants' responses to these questions, as well as other parts of the interviews.

6 Besides Andy, a few other participants noted that changing the teacher accountability approach would also change the type of person who became a teacher, implying that a self-selection effect might influence the efficacy of teacher accountability. Even so, such self-selection would not be sufficient for effective teacher accountability if the accountability instruments were at odds with the macrosystem. As noted above, several Singaporean participants said that, on a personal level, they would strongly prefer Finland's teacher accountability approach over Singapore's. Yet they persist in Singapore's 
teaching profession despite their aversion to its accountability approach, thus showing that the self-selection mechanism is not all-encompassing - unlike the macrosystem, which influences every teachers' response to accountability instruments, albeit to different degrees.

7 Besides macrosystemic trust, there are other identifiable aspects of Finland's and Singapore's sociocultural contexts that have a mutually reinforcing interaction with each country's approach to teacher accountability. As I argue elsewhere, Finnish and Singaporean interview participants had distinctly different mental models of the factors that raised and lowered their motivation, and their respective mental models were compatible with each education system's respective accountability approach (Hwa, 2019).

8 Moreover, I suspect that Finland and Singapore may both benefit from relatively strong societal consensus about certain values and priorities - perhaps resulting from small populations and relative institutional stability, among other factors. If my hunch is true, such sociocultural alignment may offer clearer orientations for context-sensitive policymaking than would be present in many other education systems.

\section{References}

Aho, E., Pitkanen, K., \& Sahlberg, P. (2006). Policy Development and Reform Principles of Basic and Secondary Education in Finland Since 1968 (No. 36871). Retrieved from World Bank website: http://documents.worldbank.org/curated/en/124381468038093074/Policydevelopment-and-reform-principles-of-basic-and-secondary-education-in-Finlandsince-1968.

Andrews, M., Pritchett, L., \& Woolcock, M. (2017). Building State Capability: Evidence, Analysis, Action. Oxford, NY: Oxford University Press.

Bovens, M. (2007). Analysing and assessing accountability: A conceptual framework. European Law Journal, 13(4), 447-468.

Broekman,A. (2016).The effects of accountability:A case study from Indonesia (pp. 72-96). In: J.Evers \& R. Kneyber (Eds.), Flip the System: Changing Education from the Ground Up (Online edition). Oxford; New York: Routledge. https://doi.org/10.4324/9781315678573.

Bronfenbrenner, U. (1977). Toward an experimental ecology of human development. American Psychologist, 32(7), 513-531.

Butrymowicz, S. (2014). Lessons from abroad: Singapore's secrets to training world-class teachers. The Hechinger Report, February 10. Retrieved 5 April 2019, from The Hechinger Report website:https://hechingerreport.org/lessons-from-abroad-singapores-secrets-totraining-world-class-teachers/.

Cerna, L. (2014). Trust: What it is and Why it Matters for Governance and Education [OECD Education Working Papers]. Retrieved from OECD website: http://www.oecd-ilibrary. $\mathrm{org} /$ content/workingpaper/5jxswcg0t6wl-en.

Crehan, L. (2016a). Cleverlands. London: Randon House.

Crehan, L. (2016b). Exploring the Impact of Career Models on Teacher Motivation. Paris: International Institute for Educational Planning, International Institute for Educational Planning, UNESCO.

Crouch, D. (2015). Highly trained, respected and free: Why Finland's teachers are different. The Guardian, June 17. Retrieved from https://www.theguardian.com/education/2015/ jun/17/highly-trained-respected-and-free-why-finlands-teachers-are-different.

Deci, E. L., \& Ryan, R. M. (2000). The 'what' and 'why' of goal pursuits: Human needs and the self-determination of behavior. Psychological Inquiry, 11(4), 227-268.

Ee, D. (2018). A Monopoly over Morality: How Moral Issues Are Publicly Resolved in Singapore (Unpublished B.A. Thesis). New Haven, CT:Yale University. 
European Commission/EACEA/Eurydice (2018a). Finland: Conditions of Service for Teachers Working in Early Childhood and School Education. EACEA, December 27. Retrieved 23 April 2019, from Eurydice website: https://eacea.ec.europa.eu/national-policies/ eurydice/content/conditions-service-teachers-working-early-childhood-and-schooleducation-24_en.

European Commission/EACEA/Eurydice (Ed.), (2018b). Teaching Careers in Europe: Access, Progression and Support. Luxembourg: Publications Office of the European Union.

EVS (2016). European Values Study 2008 - Integrated Dataset (ZA4800) Variable Report No. 2016|2. gesis, April 15. Retrieved from https://dbk.gesis.org/dbksearch/sdesc2. asp?no=4800\&db=e\&doi=10.4232/1.12458.

Finnish National Board of Education (2013). Teachers in Finland- Trusted Professionals. Retrieved from http://www.oph.fi/download/148962_Teachers_in_Finland.pdf.

Gelfand, M. J., Lim, B. -C., \& Raver, J. L. (2004). Culture and accountability in organizations: Variations in forms of social control across cultures. Human Resource Management Review, 14(1), 135-160.

Hepburn, H. (2017). Scotland eyes Singapore in 'radical' overhaul of teaching career paths. Tes, June 23. Retrieved from https://www.tes.com/news/scotland-eyes-singapore-radicaloverhaul-teaching-career-paths.

Ho, J. -M., \& Koh, T. -S. (2018). Historical development of educational leadership in Singapore (pp. 29-83). In: T. -S. Koh \& D. Hung (Eds.), Leadership for Change: The Singapore Schools' Experience. Hackensack, NJ: World Scientific.

Holloway, J. (2018). Teacher evaluation as an onto-epistemic framework. British Journal of Sociology of Education, 40(2), 174-189.

Holloway, J., \& Brass, J. (2018). Making accountable teachers: The terrors and pleasures of performativity. Journal of Education Policy, 33(3), 361-382.

Hwa, Y. (2019). Teacher accountability policy and sociocultural context: A cross-country study focusing on Finland and Singapore (Doctoral thesis). https://doi.org/10.17863/ CAM.55349.

Iyengar, R. (2012). Social capital as the catalyst for school participation. Compare, 42(6), 839-862.

Kan, Y. (2014). Your appraisal and career planning process streamlined. Contact: The Teachers' Digest, (14), 14-15.

Kim, J., Sun, M., \& Youngs, P. (2019). Developing the “will”: The relationship between teachers' perceived policy legitimacy and instructional improvement. Teachers College Record, 121(3), 1-44.

Kozlowski, K. P., \& Lauen, D. L. (2019). Understanding teacher pay for performance: Flawed assumptions and disappointing results. Teachers College Record, 121(2), 6.

Kumpulainen, K., \& Lankinen, T. (2016). Striving for educational equity and excellence: Evaluation and assessment in Finnish basic education (pp. 71-82). In: H. Niemi, A. Toom, \& A. Kallioniemi (Eds.), Miracle of Education: The Principles and Practices of Teaching and Learning in Finnish Schools. Rotterdam: Sense Publishers. (2nd revised edition).

Lawson, M., \& Martin, M. (2018). The Commitment to Reducing Inequality Index 2018: A Global Ranking of Governments Based on What They are Doing to Tackle the Gap Between Rich and Poor. https://doi.org/10.21201/2018.3415.

Lee, K. Y. (2000). From Third World to First: The Singapore Story, 1965-2000. New York: HarperCollins Publishers.

Lerner, J. S., \& Tetlock, P. E. (1999). Accounting for the effects of accountability. Psychological Bulletin, 125(2), 255-275.

Liew, W. M. (2012). Perform or else: The performative enhancement of teacher professionalism. Asia Pacific Journal of Education, 32(3), 285-303. 
Lipsky, M. (2010). Street-Level Bureaucracy: Dilemmas of the Individual in Public Service (30th Anniversary Edition). New York: Russell Sage Foundation.

Malinen, O. -P.,Väisänen, P., \& Savolainen, H. (2012). Teacher education in Finland: A review of a national effort for preparing teachers for the future. Curriculum Journal, 23(4), 567-584.

Martin, M. O., Mullis, I.V. S., Foy, P., \& Hooper, M. (2016a). TIMSS 2015 International Results in Mathematics. Retrieved from http://timss2015.org/wp-content/uploads/filebase/ full\%20pdfs/T15-International-Results-in-Mathematics.pdf.

Martin, M. O., Mullis, I.V. S., Foy, P., \& Hooper, M. (2016b). TIMSS 2015 International Results in Science. Retrieved from http://timss2015.org/wp-content/uploads/filebase/full\%20 pdfs/T15-International-Results-in-Science.pdf.

Maxwell, J. A. (2012). A Realist Approach for Qualitative Research. Los Angeles, CA: SAGE Publications.

McLaughlin, M.W. (1987). Learning from experience: Lessons from policy implementation. Educational Evaluation and Policy Analysis, 9(2), 171-178.

McMillan, C. (2017). Churchill Fellowship Report:To Investigate How Public Education Systems of Government in Singapore and Finland Develop and Prepare Leaders. Retrieved from Winston Churchill Memorial Trust website: https://www.churchilltrust.com.au/media/fellows/ McMillan_C_2015_educational_leaders_and_increased_autonomy.pdf.

Muhonen, S. (2017). In Finland, it's easier to become a doctor or lawyer than a teacherHere's why. News blog, October 16. Retrieved 22 February 2019, from The Hechinger Report website: https://hechingerreport.org/teacher-voice-in-finland-its-easier-tobecome-a-doctor-or-lawyer-than-a-teacher-heres-why/.

Murnane, R. J., \& Cohen, D. K. (1986). Merit pay and the evaluation problem: Why most merit pay plans fail and a few survive. Harvard Educational Review, 56(1), 1-18.

Nelson, L. (2016,). What the US could learn from Singapore about making teachers better. Vox, January 15. Retrieved 28 February 2019, from Vox website: https://www.vox. com/2016/1/15/10773592/teachers-singapore-shanghai-professional-development.

Neo, B. S., \& Chen, G. (2007). Dynamic Governance: Embedding Culture, Capabilities and Change in Singapore. Hackensack, NJ: World Scientific.

OAJ. (2018) Opetusalan työolobarometri 2017 [Barometer of Teachers' Working Conditions] (OAJ:n julkaisusarja 5:2018). Retrieved from OAJ (Trade Union of Education in Finland) website: https://www.oaj.fi/ajankohtaista/julkaisut/2018/opetusalan-tyoolobarometri/.

Oates,T. (2015). Finnish Fairy Stories. Retrieved from Cambridge Assessment website: https:// www.cambridgeassessment.org.uk/Images/207376-finnish-fairy-stories-tim-oates.pdf.

OECD (1982). Reviews of National Policies for Education: Finland. Paris: OECD Publishing.

OECD (2004). Learning for Tomorrow's World: First Results from PISA 2003. In: PISA. https:// doi.org/10.1787/9789264006416-en.

OECD (2007). PISA 2006: Science Competencies for Tomorrow's World: Analysis (Volume 1). In: PISA. https://doi.org/10.1787/9789264040014-en.

OECD (2010). PISA 2009 Results: Overcoming Social Background: Equity in Learning Opportunities and Outcomes (Volume II). In: PISA. https://doi.org/10.1787/9789264091504-en.

OECD (2013). PISA 2012 Results: Excellence through Equity: Giving Every Student the Chance to Succeed (Volume II). In: PISA. https://doi.org/10.1787/9789264201132-en.

OECD (2016). PISA 2015 Results: Excellence and Equity in Education (Volume I). Retrieved from http://dx.doi.org/10.1787/9789264266490-en.

OECD, \& UNESCO Institute for Statistics (2003). Literacy Skills for the World of Tomorrow: Further Results from PISA 2000. In: PISA. https://doi.org/10.1787/9789264102873-en.

Paronen, P., \& Lappi, O. (2018). Finnish Teachers and Principals in Figures (No. 2018:4). Retrieved from Finnish National Agency for Education website: https://www.oph.fi/ download/189802_finnish_teachers_and_principals_in_figures.pdf. 
Pawson, R., \& Tilley, N. (1997). Realistic Evaluation. London: SAGE Publications.

Pierson, D. (2019). Singapore's 'kiasu' culture makes FOMO look like child's play. Los Angeles Times. January 18. Retrieved from https://www.latimes.com/world/asia/ la-fg-singapore-kiasu-fomo-20190118-story.html.

Quah, J. S. T. (2010). Public Administration Singapore-Style. Bingley, UK: Emerald Publishing. https://doi.org/10.1108/S0732-1317(2010)19.

Sahlberg, P. (2015). Finnish Lessons 2.0: What Can the World Learn from Educational Change in Finland? (2nd edition). New York: Teachers College Press.

SAK (2016). The competitiveness pact in brief. SAK, March 7. Retrieved 21 February 2019, from SAK:The Central Organisation of Finnish Trade Unions website: https://www.sak. fi/en/whats-new/news/competitiveness-pact-brief.

Schunk, D. H., Pintrich, P. R., \& Meece, J. L. (2010). Motivation in education: Theory, research, and applications. In: Pearson Educational International (3rd edition, International edition). London: Pearson, Merrill, Prentice-Hall.

Sclafani, S., \& Lim, E. (2008). Rethinking Human Capital in Education: Singapore as a Model for Teacher Development. Retrieved from Aspen Institute website: http://eric. ed.gov/?id=ED512422.

Simola, H. (2014). The Finnish Education Mystery: Historical and Sociological Essays on Schooling in Finland (1st edition). London; New York: Routledge.

Simola, H., Kauko, J., Varjo, J., Kalalahti, M., \& Sahlstrom, F. (2017). Dynamics in Education Politics: Understanding and Explaining the Finnish Case. Abingdon, Oxfordshire; New York: Routledge.

Simola, H., Rinne, R., Varjo, J., Pitkänen, H., \& Kauko, J. (2009). Quality assurance and evaluation (QAE) in Finnish compulsory schooling: A national model or just unintended effects of radical decentralisation? Journal of Education Policy, 24(2), 163-178.

Singapore Ministry of Education (2018). Admissions: Returning Singaporeans: Other information. MOE, May 23. Retrieved 22 April 2019, from Ministry of Education website: https:// www.moe.gov.sg/admissions/returning-singaporeans/other-information.

Singapore Teachers' Union (2014). Performance \& expectations - The 'D' grade. The Mentor, Term, 4-5.

Singapore Teachers' Union (2015). Revisions to the performance review process. The Mentor, Term, 4.

Statistics Finland (2018). Finland in Figures 2018. Retrieved from http://www.stat.fi/tup/ julkaisut/tiedostot/julkaisuluettelo/yyti_fif_201800_2018_19693_net.pdf.

Strauss, V., \& Sahlberg, P. (2015). Teach for Finland? Why it won't happen. Washington Post, February 12. Retrieved from https://www.washingtonpost.com/news/answer-sheet/ wp/2015/02/12/teach-for-finland-why-it-wont-happen/.

Tan, K. P. (2018). Singapore: Identity, Brand, Power (1st edition). Cambridge, UK: Cambridge University Press. https://doi.org/10.1017/9781108561273.

Teo,Y.Y. (2018). This Is What Inequality Looks Like. Singapore: Ethos Books.

Tirri, K. (2014). The last 40 years in Finnish teacher education. Journal of Education for Teaching, 40(5), 600-609.

Tremewan, C. (1994). The Political Economy of Social Control in Singapore. Retrieved from https://www.palgrave.com/gb/book/9781349235124.

Tyler,T. R. (2006). Psychological perspectives on legitimacy and legitimation. Annual Review of Psychology, 57(1), 375-400.

United Nations (2017). World Population Prospects: The 2017 Revision, Key Findings and Advance Tables (No. ESA/P/WP/248). Retrieved from United Nations, Department of Economic and Social Affairs, Population Division website: https://population.un.org/ wpp/Publications/Files/WPP2017_KeyFindings.pdf. 
Vainikainen, M. -P., Thuneberg, H., Marjanen, J., Hautamäki, J., Kupiainen, S., \& Hotulainen, R. (2017). How do Finns know? Educational monitoring without inspection and standard setting (pp. 243-259). In: S. Blömeke \& J. -E. Gustafsson (Eds.), Standard Setting in Education:The Nordic Countries in an International Perspective. Cham, Switzerland: Springer. https://doi.org/10.1007/978-3-319-50856-6_14.

Velayutham, S., \& Perera, M. H. B. (2004). The influence of emotions and culture on accountability and governance. Corporate Governance, 4(1), 52-64.

Verger,A., \& Parcerisa, L. (2017).A difficult relationship. Accountability policies and teachers: International evidence and key premises for future research (pp. 241-254). In International Handbook of Teacher Quality and Policy. New York: Routledge. https://doi.org/10.5281/ zenodo. 1256602 .

Webber, D. J. (2010). School district democracy: School board voting and school performance. Politics and Policy, 38(1), 81-95.

World Bank (2019). World Development Indicators: GINI Index. Retrieved 26 February 2019, from DataBank website: https://databank.worldbank.org/data/reports.aspx?source $=2 \&$ series $=$ SI.POV.GINI\& country $=$.

WVS Association (2015). World Values Survey (2010-2014): Crossings by Country. Retrieved from World Values Survey Association website: http://www.worldvaluessurvey.org/ WVSDocumentationWV6.jsp. 


\title{
12
}

\section{DISTRUST, ACCOUNTABILITY AND CAPACITY IN SOUTH AFRICA'S FRAGMENTED EDUCATION SYSTEM}

\author{
Nomancotsho Pakade and Thokozani Chilenga-Butao
}

\section{Introduction}

The right to education in South Africa is enshrined in the 1996 Constitution of the Republic of South Africa which refers to an education system tasked with making education available, accessible and adaptable. Despite the South African government's explicit commitments to education reform in the 1990s, there are provincial education departments (PEDs) that are dealing with an education system with uneven performance and poor learner outcomes.

This chapter highlights the challenges of accountability and capacity in PEDs of education, specifically in the province of the Eastern Cape. This province underwent a process referred to as the School Rationalisation Project (SRP) which sought to facilitate an accessible and better resourced schooling system through the eradication of small and unviable schools. After some internal reflections and several attempts to rationalise in the province, the Eastern Cape Department of Education requested support from the Government Technical and Advisory Centre (GTAC) to assist with the SRP. This project was an attempt to provide access to better quality education in a province whose education department has a strong historical legacy from the apartheid education system, which still plagues the structure and actions of the department to date. Through this case study, we argue that on the one hand there is formal bureaucratic accountability that is expressed through laws and regulations, while on the other, there are uncodified practices within micro-systems of governance at the provincial and district levels that produce different outcomes to the intended goals of improved learning and teaching.

We apply the notion of codified and uncodified practices to examine this argument using the case study of the SRP and its particular implementation in the Eastern Cape province of South Africa. Yee (1997: 1012) ${ }^{1}$ describes codified 
practices as, 'Formal rules [that] are intentional social entities...within an organization context, given a textual expression, and endowed with external, often coercive, enforcement capabilities'. Therefore, codified practices are ones which are legally or formally regulated practices within an organisation or institution. However, institutions also create uncodified practices, described as 'norms, conventions, self-imposed codes of conduct, and shared understandings and expectations (i.e. informal rules) [which] are largely evolutionary ideational entities, usually uncodified and unwritten, possessing informal social and psychological enforcement' (Yee 1997: 1012). This chapter argues that given the difficulties around accountability and capacity in the South African education system as a whole, there is evidence that officials resort to using uncodified practices more than codified practices in order to implement education policy, as evidenced in the rationalisation case study that we present below.

This chapter is divided into three sections: the first section provides a brief history of the South African education system, which prior 1994 was separated into one education system for white learners and another for African, Coloured and Indian learners. The former system was implemented in the then four provinces: the Cape Province, Natal, Orange Free State and Transvaal. The latter was implemented in regions collectively known as the Bantustans. The Bantustan comprised the 'independent homelands' of the Transkei, Bophuthatswana, Venda and the Ciskei, as well as a number of 'own administrations' for Coloured and Indian people. This segregated system was based on the racist apartheid regime. The explanation of this system is integral to understanding the wider historical implications of such a system on the current education landscape in South Africa's democracy.

The second section of this chapter outlines where there were possibilities for trust, accountability and capacity in the post-apartheid education system. Here, we illustrate that there were a number of codified norms that were established in the democratic state, which informed policy formulation by the Department of Basic Education (DBE), and its subsequent implementation in PEDs, and their districts and schools. In this section, we also posit that given the enormity of the task of transforming South Africa's education system from a balkanised apartheid system to an inclusive one under the democratic dispensation, the most prevalent issues in the system are accountability and capacity more than trust. The reason for this is that first, incoherence in accountability and capacity does not create the environment to cultivate trust amongst education stakeholders. Second, there are various reasons for issues of trust and distrust in the South African education system. We provide reflections in order to show the various reasons for trust and distrust in South Africa's education system.

The third and final section of this chapter examines the case of the SRP in the Eastern Cape PED. Rationalisation is not new in the province and previous rationalisation efforts were characterised by non-compliance with the South African Schools Act (84 of 1996) on the issues of closure of schools, fragmented planning that impacted on the availability of scholar transport, teacher placements (from old to new schools), and availability of resources and infrastructure 
in receiving schools. The unavailability of scholar transport for learners moving to schools further away created distrust from communities which, in turn, fuelled the resistance against rationalisation. The need for rationalisation in the Eastern Cape is especially pressing. The Province inherited a particularly high number of very small schools from the former Transkei era, while migration to urban centres both within and outside of the province has further contributed to the proliferation of small, unviable schools. When the GTAC Project began in 2016, close to $50 \%$ of schools had fewer than 250 learners, and an additional $17 \%$ had less than 100 learners. This environment creates very difficult challenges for the future institutionalisation of the intervention undertaken by GTAC. A number of potentially valuable lessons can be drawn from the SRP, both for the Eastern Cape experience and for other provinces implementing rationalisation.

Finally, this chapter emphasises the role and significance of the mesosystem and the exosystem of the education ecology system, as per Bronfenbrenner's (1989) illustration discussed in Chapter 1 of this book. In this exosystem of South Africa's basic education system, we find the PEDs, which are integral to the implementation of education policy, while school district offices are located in the mesosystem of South Africa's basic education system. Both the PEDs and district offices are focal points in our argument and reflect the practical aspects of education provision in South Africa. Moreover, the exosystem and mesosystem are the levels wherein improvements and interventions for education are most implemented, as our case study will illustrate. By focusing on these levels, we extend our analysis beyond the interaction between learners, parents and teachers and move towards other instrumental actors and institutions in South Africa's education provision. The instrumental actors and institutions are provincial officials, district officials and education intervention strategists.

\section{Fragmentation and transition: A brief overview of the South African education system}

South Africa's current education system is a result of and a reflection on significant efforts to create a public administration representative of all South Africans and to meet democratic and development goals to provide inclusive, quality primary education. Before the formation of the current system of education in 1994, there was minimal trust, accountability and capacity in the apartheid education system. The apartheid education system was built on several education systems following its missionary school predecessor. The latter espoused less discriminatory efforts towards racially inclusive education while the apartheid regime promoted separate development for whites, Indians, Coloureds and Blacks which translated to better investments in white minority education (Behr, 1988; Thompson, 2000). As such, the Eiselen Commission was formed in 1949 (although preceded by the 1947 Sauer Report) to investigate how to restructure the education system for the particular incorporation of Black Africans. ${ }^{2}$ Following the Eiselen Commission, the education of black Africans was centralised into the Department of Native 
Affairs. When the Bantu Education Act was formalised in 1953, about 90\% of the African schools were state-aided mission schools. The function of education for black Africans was moved to the Department of Bantu Administration, Development, and Bantu Education. The education of Coloured (Education for Coloured People Act of 1965) and Indian (Indians Act of 1969) children fell under the control of the provinces which administered white minority education but later moved to 'Own Affairs' departments situated in those communities.

The restructuring of the education system from 1948 to 1990 under the apartheid regime coincided with a number of legislative changes such as the Native Laws Amendment Act 54 of 1952 and the Reservation of Separate Amenities Act 49 of 1953, which restricted the access of Africans to urban areas for 72 hours and provided separate amenities for different races in different spaces, respectively. These two acts are illustrative of the spatial location of Africans, specifically in rural homelands and township settings. Hence, the movement of education from the Department of Natives Affairs to the Department of Bantu Administration and Development and then Bantu Education was not coincidental because it emphasised the spatial arrangements of segregation that would come to characterise the apartheid state in general, and the administration and resourcing of education to the disadvantage of the majority of the population.

The political result of this was that education for white learners at the time was well funded and produced the desired outcome of developing their education and training. Furthermore, as a regulated practice in education but more broadly under the apartheid segregation, education for white learners, also known as Model $\mathrm{C}^{3}$ education, was superior to the underfunded, biased and limited education for Black African, Coloured and Indian learners. As a result, even in South Africa's current inclusive democracy, racist tropes about who is better educated, depending on the history of the resourcing of schools during apartheid, remain. The administrative result of the apartheid education system underpinned by the racist ideology is that, apart from white schools and their governance, the system had approximately 19 different education systems in the Bantustans (Letsoalo, 1995; Ngoma, 2007; Van der Berg, 2007). Each of the education systems in the Bantustans had its own administrative, financial and operative patterns as well as its own institutional, environmental and political factors that influenced its education outcomes. The Bantu education system comprised the following:

1. 'The four independent homelands, Transkei, Bophuthatswana, Venda and Ciskei, each with its education system

2. The six governing territories, Lebowa, Gazankulu, Kwandebele, QwaQwa, KwaZulu and Kwangane

3. The three different own affairs education departments for whites, Coloureds and Indians

4. The two general affairs education departments for Education and Training and the National Education' (Letsoalo, 1995: 1). 
Therefore, the Bantustan education system was subject to apartheid decentralisation, as a form of state formation, as much as the rest of South Africa (Bardhan and Mookerjhee, 2006). During the transition to democracy, a process that began in the 1991 negotiations for a peaceful transition, all existing and separate education departments were amalgamated. This meant that education departments in the Bantustans and provinces had to merge into one national department and nine oversight provincial departments in each of South Africa's nine provinces. Currently, there is one department, nine provincial departments and 86 districts (DBE, 2019). Despite the reform efforts, as early as 1999 the state limited expenditure in education even though education is positioned as a necessary human resource for the political and economic development of the country. As Languille (2018) points out, the public schools are empowered to determine fees for the school, thereby creating elite public schools financially inaccessible to the majority of South Africans. This class demarcation reinforces the gap between affluent and no-fee paying schools (See also Fiske and Ladd, 2005). The Eastern Cape between 2006 and 2013 experienced a sharp increase of learner migration to independent schools.

The enrolment of learners under Bantu Education was determined by the Native Commissioner in consultation with the Bantu Education Inspector who had the power to recommend candidates. The Act also had a special provision for the sons of chiefs to train them as future leaders (Ramoketsi, 2008). Locally, access to schooling was facilitated via the elite groups of chiefs, headmen and other community leaders as school committee and board members. Additionally, learners to be considered for admission would belong to an ethnic group from the surrounding communities that served the school. The enrolment numbers were kept as low as possible and the curriculum remained elementary with emphasis on producing manual labourers. Between 1953 and 1965, the enrolment of Bantu education schools doubled and they were overcrowded: primary school enrolment increased from 852,000 to 1833,000 while secondary schools also grew from 31,000 to 66,000 (Ramoketsi citing Troup 1977). By 1979, 3484,329 African children were listed as learners. The expansion was contained: white compulsory education with the state expenditure 10 times more per capita than on African learners (Thompson, 2000). From trained teachers, infrastructure and textbooks, Black schools in the urban townships and Bantustans were severely under-resourced through the race-based funding scale (Christie, 1985). This was as a consequence of the growing economy which demanded semi-skilled migrant workers with minimum basic education for the rapid industrialisation of the markets and the rise of population growth: the Bantu education provided exactly that. As such by 1991, in Black schools, the infrastructure shortfall was at 29,000 classrooms at the primary level and 14,000 at the secondary level. By 1994, approximately one in three teachers were underqualified (Fiske and Ladd, 2005) or 46\% were under- or unqualified (South African Institute of Race Relations, 1997).

Thus, Bantu education became a product of the requirements of political and economic structures of the country, intended not to raise the abilities of 
Africans to their full capacity but to facilitate access to cheap labour under the slogan 'teach the native to work'(Nokwe, 1954; See also Christie and Collins, 1984). The teacher-learner ratio was $1: 42$ on average but in the former Transkei, as the anomaly, it stood at 1:70. ${ }^{4}$ Between 1953 and 1993, the teacher-learner ratio in black schools was 1:50, 1:70 in extreme cases, while the white schools had a maximum ratio of 1:25 (Fedderke et al., 2000). The outcome of this was the low performance of Black, Coloured and Indian learners. In addition to this, the resourcing of schools in Bantustans, in particular, was highly politicised. That is, these schools functioned in environments where financial resources were dependent on the relationship between Bantustan leaders and the apartheid government. For instance, the Lebowa Department of Education, then part of the Northern Transvaal Education Department and now a predecessor of the Limpopo Department of Education, was a weak institution with an equally weak administration (Jacklin, 1994: 1). Furthermore, the Lebowa Department of Education was 'operating in the poorest region in the country with minimal resources, capacity and legitimacy' (Jacklin, 1994: 1). In the midst of this, the Lebowa Department of Education was riddled with mismanagement and corruption, which residents in the community protested against (Jacklin, 1994: 2).

Similarly, the Gazankulu Education Department of Education - also within the Northern Transvaal Education Department - was characterised by mismanagement and corruption that was 'rampant, undisguised and relatively unchallenged' (Jacklin, 1994: 2). However, unlike other Bantustan education departments, Gazankulu was allocated more resources by the apartheid government because it had a higher number of 'qualified teachers' (Jacklin, 1994: 1). Moreover, the institutions and bureaucracies of the Bantustan education system each had their own patterns and ways of working (Ngoma, 2007). Therefore, the levels of trust, accountability and capacity in the Bantustans were low at most and varied at least.

\section{Legacies of fragmented trust, accountability and capacity in South Africa's education system}

At the end of apartheid, there was a large, inefficient and race-based education system. More importantly for this book and chapter, there were mixed levels of trust, accountability and capacity in this highly fragmented apartheid education system. The education system as a whole, from the micro to the macro systems, was geared to advantage or disadvantage learners according to their race and geographical location. Within that system, mostly white schools were $80 \%$ state resourced (Dekker and Van Schalkwyk, 1996) financially, well-staffed with teachers and the necessary learning materials needed for education. In democratic South Africa's education system, it is these schools that still maintain their reputation for high levels of trust, accountability and capacity.

A case in point that illustrates the interplay of these three variables is the notion of learner migration and urbanisation based on the parent's choice and 
perceptions of the schools. For instance, Msila (2009) identified three kinds of choices: first, the voice option which seeks to change the school, second, loyalty as a default position in the absence of alternatives or means to choose otherwise and third, the exit option that has learners' move to the suburban former White, Indian and Coloured schools, seen to be more effective than Black rural or township schools. The third option is the frequently exercised one, even though authors such as Msila have documented intra-township migration to better performing schools. Sekete et al.'s (2001) study on learner migration indicated that the migration is one-way, towards better resourced schools. Furthermore, the exit option introduces a financial burden on the parent, which may include payment of fees, transportation and other compulsory extracurricular activities. As a result of this migration, rural and township schools inhabit empty schools commonly referred to as 'white elephant schools', which have empty buildings or small schools with below average learner enrolment numbers and teachers. Migration to better schools is caused by high levels of poverty and unemployment in people's resident or indigent provinces.

The case of learner migration from schools that were under-resourced during apartheid shows a lack of trust in education provision in these schools. These schools were established on an unequal footing with Bantu education, thus causing distrust in the ability of these schools to provide a good education. This distrust was a direct result of the lack of capacity in these schools. And, thus, began a vicious cycle of distrust, lack of capacity and lack of accountability in a fragmented education system, which continues today.

In terms of the trust, former White schools continue to enjoy institutional trust, as outlined in Chapter 1, because historically they have the resources and status of providing better education in South Africa. These schools are known as former Model C schools, which generally 'represent high-quality teaching, better discipline, safety; a chance to better oneself through one's children' (Battersby, 2004: 281). Furthermore, the high-quality teaching in these former Model C schools continues partly as a result of unequal resourcing and privilege during apartheid and partly because the legacy and institutional fortitude of these schools remain. This indicates a high level of capacity in these former Model C schools. In terms of the trust, parents continue to trust former Model C schools because of the high levels of capacity demonstrated in these spaces. Parents' perceptions about former Model C schools are that they are a 'site for fast-tracking a way out of historical disadvantage...because they believe it will provide their children with opportunities that they themselves did not have, opportunities to be counted equal with white children on entering the workforce' (Battersby, 2004: 280-281).

These high levels of trust are not only reserved for former white Model C schools but also other schools with generally high levels of capacity due to resourcing during apartheid. As a result, 'Children from black African communities are sent to historically Coloured schools, children from poor and gang-ridden Coloured communities are sent to schools in wealthier coloured suburbs, filling the gaps left by children from these areas leaving to go to Model C schools' 
(Battersby, 2004: 281). In terms of accountability, these schools are efficiently run through their school governing bodies (SGBs) and are accordingly granted extensive powers over their finances and operational procedures. These schools are currently known as Section 21 schools in South Africa, based on that section of the South African Schools Act of 1996 (SASA, 1996) and have integrated the migrant black learners from township and suburban dwellers.

Therefore, capacity and trust in the education system, both during apartheid and in other areas of the system of the current democratic South Africa, are highly racialised due to the direction of resources from the apartheid to White, Coloured, Indian and Black schools, and in descending order. However, despite these high levels of capacity and trust, there is a more complex issue emerging around racial integration at former Model C schools in democratic South Africa (Battersby, 2004; Soudien, 2010; Robinson, 2019). Thus, the education landscape has been shaped by the apartheid spatial legacy where schools with a high trust dividend attract learners from under resourced areas - even if it means that they are moving from no-fee schools to fee-paying schools. In addition to this, there is the pattern of contemporary economic migration, where families of learners move to areas with better service delivery and employment opportunities which are most likely to be in urban centres, the coastal line for the Eastern Cape. What they leave behind, are a number of small and unviable schools and at worst, empty school buildings that become vandalised if not handed over to the Department of Public Works as legislated.

This background to the current education system in South Africa matters because it shows how trust, accountability and capacity can differ significantly within an education system based on the political and social conditions of a country. The background of the apartheid education system also matters because it should cause us to question the aggregation or disaggregation of trust, accountability and capacity in the current education system. This is evident in the amalgamation of all the education departments during the transition to democracy. The amalgamation revealed that among education bureaucrats in provincial head offices there were high levels of distrust between white and black bureaucrats and between black bureaucrats from different Bantustans (Ngoma, 2007). Capacity and accountability were low too. Finally, this background matters because it indirectly shows the potential for trust, accountability and capacity in the reformed South African education system. The potential for trust is available from the micro to the macro level as the current education system is pursuing quality education for all learners, based on the principles of equality, access and social welfare.

\section{Possibilities for trust, accountability and capacity in the post-apartheid education system}

During the transition to democracy in South Africa, there was an opportunity to create a space for trust, accountability and capacity within the new education system as part of the transformation, redress agenda. Like many of South Africa's 
administrative and public policy changes at the time, the national education system underwent significant top-down and political change. The political change was primarily that of an inclusive and democratically based state. Also, along with this, the national education system experienced significant changes including the formalisation of the single, non-racialised education system as well as the reorientation of school governance and funding (DBE, 1995). The reformation of the education system in South Africa paralleled the state formation that was taking place in the country at the time. Both these processes were aimed at nation-building, policy renewal (Booysen, 2001), restructuring and transformation (Ncholo, 2000), and a general alignment with the Constitution's Bill of Rights and public administration values (Constitution of the Republic of South Africa, 1996).

At the provincial level, the reformation of the education system in South Africa was more complex and challenging. This is because, as mentioned earlier, the changes that needed to take place to align with the national education system's reformation were ones that involved the former Bantustan and White schools and their districts amalgamating under the new provinces to form a new education system. The challenges of this amalgamation revolved around different institutional legacies colliding; varying degrees of administrative capacity; multiple factions and their logic being forced to cooperate; and sometimes, the perception that the top-down process of reformation was flawed (Ngoma, 2007). Other transformative interventions included an increase in expenditure in programmes such as infrastructure (school renovations), fee subsidy, school nutrition and training of teachers. All these factors contributed to the reduction of teacher-learner ratio from 1:47 in 1994 to 1:35 by 2000 (Department of Education South Africa, 2001). Acemoglu and Robinson (2006) argue that political transition relies heavily on the idea of 'credible commitment' by the government. The challenges remain, as the number of dropouts, particularly in the secondary schools, continues to grow, with a reported $50 \%$ between Grades 10 and 12 (De Kadt, 2011). Despite these issues, there were and still are possibilities for higher levels of trust, accountability and capacity to take root in South Africa's education system.

One of the most apparent areas for higher levels of these factors was at national level, with the reformation and the representation of education through the creation of a single and national DBE. Like most national departments in South Africa, the DBE formulates policy, collates and monitors data and is supposed to maintain quality, accessible and equal education for all learners. The role of the DBE is, thus, to provide accountability in education while overseeing trust and capacity in PEDs, districts and their schools. Second, the PEDs role is then to implement national education policies at provincial level through teaching and learning; the provision of learner teacher support materials (LTSM); maintaining the provision of teachers as well as maintaining a functional provincial head office. Therefore, to do this, PEDs require a high level of capacity, and they have to oversee capacity in districts and their schools. 
It is important to note that the South African education system strives to be a hierarchical one, as outlines in Chapter 2 of this book. That is, it is an educational system that is "tightly managed from the centre with strong, topdown control of recruitment, promotion, curriculum and content of classroom instructions', as per Levy et al. (2018). Such hierarchical education systems also have relationships of 'command and control', with high levels of dependence on the state regulation of school quality through national standards and regulations. For instance, through rationalisation, the incorporation of community inputs through the public hearings and submissions are limited by the authority of the MEC who rests with the decision to close or maintain a school. As such the community's role is not on the basis of consent but consultation.

A third possibility for trust, accountability and capacity to take root is through the PEDs ability to implement auxiliary but necessary national education policies such as transport for learners, and the National School Nutrition Programme (NSNP) at the district and local level. The implementation of these policies not only requires a high level of capacity but also establishes an avenue for transparency between various internal and external stakeholders. This means that there needs to be a level of trust between schools, parents, PEDs and the DBE as well as the ability to develop mutual accountability between these stakeholders under the promise to access quality-free compulsory basic education.

A final possibility for higher levels of trust and accountability is through districts and their schools. Schools and districts are at the frontline of education provision and thus their ability to manage daily processes in schools, as well as manage the expectations of learners and their parents, is an ever-present one. However, this outline of the national, provincial and local levels of governance in education is part of a codified system of education. Uncodified practices also exist within PEDs and districts as they make trade-offs between their resources, priorities and capabilities in the large, and complicated education system. To further complicate the issue of capacity and accountability, the following section focuses on policy implementation challenges in the rationalisation process and intervention. District officials are administratively accountable to PEDs as well as the school and community. As this chapter focuses on the SRP implementation at the district level, the ensuing analysis enables a reading of the institutional interplay of these trust, accountability and capacity.

\section{Interventions in the Eastern Cape: The Schools Rationalisation Project (SRP)}

The Eastern Cape is an ideal case for understanding these dynamics of the South African educational system because it has some of the lowest learner outcomes in the country, coupled with a high poverty rate and, historically, is one of the prominent examples of apartheid spatial legacies. These three factors collide in this province to create a fertile ground for education reform, and yet the reforms have been stifled by a number of factors including a lack of capacity and accountability. 
PARI worked in a team of experts for 3 years in the SRP (Aug 2016-May 2019) and a number of minutes and interviews were collected during the project's life cycle to produce a case study documenting the implementation of the intervention. Through the SRP example, we can identify the tension between codified education reform and uncodified practices as a way to think through how trust, accountability and capacity manifest in a fragmented system.

\section{Background to the Eastern Cape PED}

The education system in the Eastern Cape, like the rest of the country, is shaped by colonial and apartheid racialised segregation; the two regions of the former Transkei and Ciskei in the Eastern Cape had their independent education institutions until the post 1994 education reforms that consolidated these two administrations. As a consequence of this spatial legacy, local communities would petition government or traditional authorities to establish a school in their area producing a landscape of rural communities that each had their own primary schools, far and scattered across the province. Post 1994, the economic migration to urban centres has also contributed to the emergence of underutilised, small schools in the rural areas. The Eastern Cape has about 5554 public schools with $15.4 \%$ of national enrolment. Relative to national averages and trends, the province has around 1849 schools too many (ECDoE Annual Report, 2015). Data from $2014^{5}$ and $2016^{6}$ demonstrates that there is still a high concentration of small schools. Some of these schools have large physical structures, but low enrolment. ${ }^{7}$ Furthermore, the Eastern Cape is also characterised by $56 \%$ of No Fee schools (Bloch, 2010), reflecting the poverty and inequality of the province. The Eastern Cape Department of Education's (ECDE) 2015 Annual Report revealed a high number of small and unviable schools which made it difficult for the department to resource the schools adequately. In addition, the Eastern Cape generally has the highest net migration statistics of the nine South African provinces. ${ }^{8}$

Cole (2018) further details the incremental numbers of learner migration within the province, noting that the migration is from rural to urban townships and suburban areas around the coastal lines are seen to be economically more viable. Furthermore, Motala and Pampallis (2001) argue that despite the attempts to redress resource allocation under the Norms and Standards for School Funding, poorer schools still face funding deficiencies compared to urban schools. This also relates to weak capacity in financial management within PEDs. Similarly, Soudien (2004) points out that the reverse of immigration to black schools has never happened in the history of the country. Thus, rationalisation comes from an analysis of poor educational outcomes within the context of a system that was chronically underfunded and arguably continues to be underfunded given the Bantustan and apartheid legacy along with the skills capacity for administration.

The SRP aimed to address the effects and consequences of the historical legacy just outlined. Rationalisation is a national DBE programme which was initiated in 1996 with the enactment of various legislation, formalised through 
the Ministerial Committee on Rural Education in 2005, and which was supposed to have been finalised in 2012 (Directorate Rural Education, 2009:7). Rationalisation is intended to consolidate the education landscape by reducing the number of small schools deemed unviable to improve resource allocation towards quality access to teaching and learning centres. More importantly, the reduction in the number of small and unviable schools allows a full education curriculum to be run daily. Instead of having one teacher teach multiple grades of learners in one class, it allows for the creation of larger, better resourced and accessible schools. Thus, rationalisation streamlines multiple administrative processes around teacher post provisioning, scholar transport, the NSNP, providing LTSM and others. Some of the provinces with large numbers of these small schools that are targeted for rationalisation are the Eastern Cape and KwaZulu-Natal.

Rationalisation of schools in the Eastern Cape, therefore, provides opportunities for high levels of trust, accountability and capacity of education provision in this province. These possibilities are presented by the policy's intention to amalgamate rural schools and thereby enhance available education resources and increase the accessibility and efficiency of education in rural areas.

The SRP in the Eastern Cape also illustrates the depth of transition and overhaul required in a fragmented system. As outlined earlier in this chapter, the transition of the education system from apartheid to democracy was mainly a national effort, comprising the amalgamation of the fragmented system and confronting the legacies of this system. In addition to this, the nine provinces in South Africa had to address regional and local legacies of apartheid's fragmentation. For the Eastern Cape in particular, and similarly to other large rural provinces, rationalisation sought to address this spatial legacy of education provision.

\section{Codified regulations for the SRP}

The SRP comprises three aspects of rationalisation, which are codified practices for closure, merger and the realignment of schools, which are set out in writing. The first type is the closure of a school, according to Section 33 of South African Schools Act 84 of 1996 (SASA). This section of the SASA stipulates that schools selected for closure should be notified by letter from the PED, public hearings should be held, documented and submission of representations made, a report with recommendations on these should be provided by a district official to inform the Member of the Executive Council's (MEC ${ }^{9}$ ) final decision. Notice for closure should be gazetted in government media and teachers and learners should be transferred to the identified nearby operational schools.

The second aspect of rationalisation is a merger, according to Section $12 \mathrm{~A}$ of the SASA, whereby following the selection of schools to be merged, a notice of merger is sent to the school and SGB to activate the submission of representations to the district office. Similar to the closure process, a report with recommendations is submitted by the district office to inform the MEC's decision. Once the MEC makes a decision, a notice of merger is published in the newspapers. 
The MEC hears appeals and oversees the necessary planning before administrative adjustments are activated. Key to the process is the establishment of an interim SGB where other details, such as the transfer of enrolment of learners, are finalised.

The third aspect of rationalisation is the process of realigning schools, as per the Regulations Relating to Minimum Uniform Norms and Standards for Public School Infrastructure, discussed further later. Following the selection of schools for realignment, provincial and district officials produce a report outlining the process for realignment, following which the district office and the PED decide to realign the school and give notice to the SGB and the transfer of learners and teachers takes place.

The Regulations Relating to Minimum Uniform Norms and Standards for Public School Infrastructure have created a clear legal position on what constitutes a small or large school. Regulation 5(3) (ii) provides that a small primary school should consist of a minimum capacity of 135 learners while regulation 5(5) (i) provides that a small secondary school should consist of a minimum capacity of 200 learners. Section 5(2) and Section 5(4) stipulate that a streamlined system of schooling should be maintained with 8 years of primary schooling (Grade R-7) and 5 years of secondary schooling (Grade 8-12). Through the Imbewu Project, it was noted that unlike the national norm where primary school starts from Grade R-7 and secondary starting from Grade 8-12, Eastern Cape has the following school system:

- $\quad$ Primary schools (lower) - Grade R-3

- $\quad$ Primary schools (higher) - Grade 4-7

- Secondary schools (junior) - Grade 7-9

- Secondary schools (senior) - Grade 8-12; Grade 10-12

- Combined schools - different combinations of primary and secondary schools (Grade R-12; Grade R-9).

By 2011 an estimate of $66 \%$ of schools in the province were classified in quintiles 1 and 2 which is the poorest and lowest end in the scale of 1-5 quintile categorisation. Generally, poorer households are more likely to require state support in the form of services like scholar transport. When small schools are closed down through rationalisation, increased burden of resources, such as infrastructure adjustments and scholar transport, falls on the PEDs. The Education Management Information System (EMIS) data shows an increase in the number of small schools between the years 2009 and 2012. Most of these schools are located in the former Transkei.

The recommendations that framed the SRP were sourced from (1) the 2005 Ministerial Committee for Rural Education which set the national tone through the Guidelines for the Merger and Closure of Rural and Farm Schools (DBE, 2012) and the provinces were left to produce detailed plans; (2) the ECDE commissioned research from Imbewu Project (2005-2008), which provided analysis for the provincial context on rationalisation; (3) between 2009 and 2010, the ECDE 
worked closely with the Committee on Rural Education to conceptualise the rationalisation process for the province. In 2012, the concept became formalised as the Provincial Framework For Rationalisation, Re-Alignment, Merger And Closure Of Public Ordinary/Special Schools In The Eastern Cape Department Of Education and; (4) the recommendation by the Council of Educators, which recommended school prototypes, thereby positioning realignment of schools as a necessary element of rationalisation.

The rationalisation and realignment of the school landscape in the Eastern Cape can be understood as a restructuring intervention both at the systemic and school levels. This analysis of learner performance shows a correlation between performance and small and unviable schools. Furthermore, the recommendations to rationalise and realign the education landscape in the province is underpinned by the need to adhere to national standards and norms (infrastructure, resources and learner numbers, etc.) which has implications for the effective and responsive distribution of resources within the education system.

These codified practices for the SRP illustrate attempts by the South African government to standardise the outputs and outcomes in a system that remains fragmented due to unequal inputs during apartheid. To this end, and as described by Levy et al. (2018), carrying out bureaucratic tasks becomes a measure of successful standardisation and results in hierarchical coordination and the consolidation of trust, accountability and capacity. The analysis in this chapter demonstrates that standardisation, as a process of hierarchical coordination, is difficult to achieve because it is complex with competing procedures and resource intensive, and the district officials who implement the SRP resort to uncodified practices for implementation.

\section{Uncodified practices in the implementation of the SRP}

The first attempt to rationalise schools in the Eastern Cape began between 2012 and 2015, using the 2012 Provincial Guidelines. Three parallel processes characterised the implementation of the SRP:

Practice A: Closing non-operational schools led by the Eastern Cape PED. These are shell schools considered 'unofficially closed' because of the procedural non-compliance that hinders the the PED's education management information system (EMIS) from deregistering such schools. Therefore categorised as 'pending closure' dependent on the official closure by EMIS as delegated by the MEC. Thus EMIS suspend the resource allocation to schools pending closure.

Practice B: Meanwhile, district offices are also closing non-operational schools, whereby district officials quietly implement rationalisation in their own areas. At the district level these ad-hoc practices of school closures relied on what PARI refers to as the 'operational power' 10 of circuit and district managers, who facilitate learners' and teachers' movements based 
on pressing demands in their area, submitted by principals and upon negotiation with parents to move learners. Nevertheless, these sometimes innovative and well-intentioned ways of navigating the administrative processes for rationalisation on the ground, sometimes adversely contribute to instability in the system (including the management of data on assets, teacher and learner numbers and so on, which are important for planning and resource management purposes). Similar to Practise A, the school remains registered with dwindling learner and teacher numbers categorised as pending closure in the EMIS system, until the community and district officials agree to move i.e. 10 to 50 learners left.

Practice C: This refers to the GTAC SRP which uses the CSLPs as the cornerstone for an improved comprehensive and coordinated planning that centres on the district capacity to implement.

The first two practices feed into each other and create two non-compliant parallel practices of closure which have not led to the de-registration of schools via the MEC and EMIS, as codified. During this period, approximately 504 schools were unofficially closed. ${ }^{11}$ The initial $2012-15$ rationalisation process showed that the challenges confronting school closures were based on the scholar transport and the infrastructure ${ }^{12}$ backlogs. The critical success factor in this period was the leadership role of district directors in closing some schools, although the process was reactionary and not driven by an overall district plan.

This reactionary approach was further weakened by a lack of compliance with the Rationalisation Guidelines and dysfunctionality of the Provincial Coordinating Team (PCT) and the District Implementing Teams (DIT). The rationalisation process became limited with regards to legislative compliance such that there was incomplete implementation of the Section 33 or 12A processes due to factors such as lack of information about the codified practises that are in place. The result of this incomplete compliance was 'white elephant' schools, which are vacant school buildings, that were not officially handed over to PED nor the Department of Public Works as outlined under the asset management procedures of the department, because the official deregistration of closed or merged schools can only be finalised once all the procedural steps in the codified practice have been carried out and authorised by the MEC. Therefore, the result of the uncodified practices is an increasing number of 'white elephant' schools as well as 'double parking' of teachers. Double parking refers to the process of teachers following learners to rationalised receiving schools, when their home school is closed or merged; this creates an excess supply of teachers in the receiving school.

By the time the GTAC intervention was implemented (2016-19), it became the third practice that tried to consolidate these parallel systems. The GTAC model facilitated the transfer of learners and teachers through a planned and phased approach, driven by data in the comprehensive Circuit School Landscape Plans (CSLPs). Additionally, the process guided the Standard Operating Procedures 
that outline all the steps for the three aforementioned scenarios for rationalisation in line with legislation. The GTAC systems and tools distinguished this phase from the previous ECDE - one that had less coordinated planning and limited tools. Due to multiple rounds of interventions and approaches, there has been a proliferation of processes and leaderships associated with rationalisation. This has created frustration for some district and circuit officials and has led to ad-hoc approaches to rationalisation being followed on the ground. More generally, uneven and often broken administrative systems (for example, data collection) deepen the complexity in this rationalisation process in the province. This administrative environment also has insufficient coordinated planning for rationalisation in two key areas - scholar transport and school infrastructure.

At the PED level, the uncodified practices of the SRP are compounded by newer projects and programmes that reorient the planning and implementation of the ECDE. Therefore, there is continuous change and restructuring at the provincial level, which destabilises SRP further because these changes often occur without the effectiveness of existing interventions having been tested or with competing demands on the officials, which further adds to the incoherence of project implementation. For instance, the SRP unfolds under the Service Delivery Model approach, Strategic Plans, Data-Drive Districts and the Accelerated Schools Infrastructure Development Initiative amongst other strategic initiatives that are conceptually interwoven but, in practice, operate in silos. During the School Rationalisation, the department had 242 circuits in 23 districts coordinated through three clusters at the provincial Head Office, it currently has 220 circuits in 12 districts under two clusters post the amalgamation. This process of district rationalisation delayed the finalisation of the CSLPs. The creation of parallel systems has an adverse long-term effect on the overall project. Jansen (2004) maintains that complexity within the education system is such that the system requires much coordination of various actors who possess some degree of autonomy and relating to this, is the high level of interdependence, which all require alignment of the policy strands.

\section{Conclusion}

The case of rationalisation includes the post-apartheid transition, continuous restructuring within the education system, and how the historically fragmented system shapes interventions. The combination of institutional priorities, strategies and interventions aimed to improve the education system, have the potential (as they are simultaneously implemented) to exert what McIntyre and Klugman (2003) describe as transformation fatigue characterised by isolated initiatives, which potentially could divert work from the overarching goals. Transformation, therefore, requires various forms of accountability that consider delegation, the flow of resource and support, information management and motivation. As such, weak institutional capacity can be the result of incoherence in accountability relationships within the organisation, in particular, the management relationship. 
Weak implementation of projects can be symptomatic of weak elements of the system of accountability relationships in which an organisation is embedded (Andrews et al., 2017). Thus, the uncodified practices, which result in administrative challenges such as 'double parking' for Human Resource and post provisioning processes which affect teaching capacity at the school level, are amongst the typical ways in which accountability can become incoherent.

Districts, as intermediaries between National and Provincial Departments on the one hand, and schools, on the other, are required to implement policy by monitoring progress, as well as to provide support to schools but yet with limited managerial autonomy. Added to this, circuit offices are virtual with limited staff capacity, in that they are generally located in the District Office, only staffed with a circuit manager. Districts have become the locus where much of this information is demanded from schools for reporting purposes up to the system - the case of rationalisation points to the hyper-congestion of competing and parallel demands at the district level. Moreover, at the different operational levels when there are multiple interpretations of the intervention simultaneously at play - which was the case with the multiple rounds of rationalisation interventions - this contributes to uncodified practices. The SRP invested in central planning through the identified structures as per the rationalisation guidelines. This central planning system used the bottom-up approach of feeding data from the districts to the provincial office. However, the recipients at the provincial level would distribute the data, but this would not activate the codified processes of closure.

The district interfaces with various education stakeholders and positions circuit managers as responsible for the rationalisation demands without the means to allocate budget to the infrastructure of scholar transport community concerns. The issue of 'closing schools' or 'failure to deliver' scholar transport or deploy (i.e. Maths) teachers, bring together the political reading of education promises unfulfilled by political heads-nationally, which in turn casts doubt on the ability of the provincial government to deliver services. When the Superintended General (SG) or other senior officials concede to unbudgeted demands due to political expediency, the technical consequences of these are no different from uncodified practices used by district officials to navigate the rationalisation of schools. These threaten a stable financial management through proper budgeting, control and reporting as the challenges of expenditure exceeding the projections due to unscheduled payments remain. Other factors maintain these limitations such as the communication challenges between and to districts, and the dishonouring of confirmed programmes and agreements. ${ }^{13}$

Although there has been some critique on focusing on learner enrolment numbers in determining the restructuring of the education landscape, the enrolment numbers are indicative of the stark inequalities in learner performance amongst the different school quintiles. The disparity in resource allocation and the migration from low-to-high performing schools widens the performance gap. Additionally, this points to the provincial department's ability to facilitate 
access to quality teaching and learning. The public trust in the education system has been declining over the years such that interventions like rationalisation are met with suspicion, partly due to the limited communication on rationalisation with both internal and external stakeholders which points to an erosion of accountability.

The GTAC team influenced the discourse of rationalisation and produced data-driven planning tools, with flexibility for future change that is dependent on maintenance and timely updates. Coordination remains central to the mainstreaming of tools and its data. The coordination requires a knowledgeable and skilled team, the department of education both at the district and national level often absorbs teachers as employees who learn through experience the administrative-governance practices in the department. This points to the limited technical expertise for complex interventions such as rationalisation but this is not to undermine the critical contribution that emerges from the teaching experience. Nonetheless, capacity enables service delivery which in turn improves trust amongst the education stakeholders at different levels of the education chain; this then opens up the possibility to develop accountability. At the same time, accountability is essential for capacity building. Additionally, this responsive accountability, as argued by Andrews et al. (2017), is not a matter of logistical implementation addressed through training or skills capacity building, and technical capacity but we argue illustrates the overall organisational capacity as a question of coherence and mainstreaming of projects (indicative of the bureaucratic and political interface).

The ECDoE 2016/17 Head Office Operational Plan reflects that, amongst its key challenges, dishonouring of decisions pertaining to efficient administration through good corporate governance and management, and the lack of capacity in internal audit, which show the limitations in the internal accountability system. The technical documents with the symbolic championing of the SRP by the SG are not met with the timeous and consistent application of the project further reinforcing the lacking confidence in the ability of the department to deliver its service. Hence, the notion of distrust. The many announced rounds of rationalisation have limited legitimacy in the public, as Andrews et al. (2017) put it 'the external accountability is not driven by accounting or detailed measures of cost effectiveness or proven impact or reducible to precise figures, but they have to continually prove to key constituencies that they work, and if these external actors no longer believe the organization's account then they lose traction with their internal agents and external constituencies no matter what the accounting says'. Thus, the technical and political mechanisms are intertwined.

Apart from availability of resources, the sustainability of rationalisation hinges on two main processes: the ECDoE's adoption of the institutionalisation framework and the actual implementation of the rationalisation plans, both produced as part of the intervention for a 10-year phased implementation approach. Institutionalising rationalisation beyond the SRP intervention will rely on several factors such as improved coordination for the planning and 
implementation of the work along with the delegation of authority and compliance by the implementers at the district level. What the case study illustrates is that access to free basic education necessitates the reduction of financial barriers in order to facilitate access; hence, the scholar transport, teacher placement and adherence to infrastructure norms and standards become integral in the rationalisation process. While efforts to implement policy are underway, the context, that is regional and other inequalities, continue to characterise the administrative (in)efficiency and (in)coherent policy implementation. The assumption is that learner improved achievement may be cultivated by the availability of resources and responsive allocation thereof. However, resources are part of the demand on capacity as the redistribution/reallocation of funds is not a guarantee of improving performance. Van der Berg (2007) maintains that it is one thing for a department/programme to have resources at its disposal but quite another for it to use the resources effectively. The dynamics of management factors required for the efficient and effective use of resources points to planning, monitoring and oversight as underlying features necessary for responsive accountability and administration.

\section{Notes}

1 See also Goodin and Olsen (2013).

2 We use 'African' as encompassing the population categories that were classified as nonWhites by the South African Population Registration Act No. 30 of 1950 and which did not form part of the Tricameral parliament system in 1984. In this chapter, Africans are those who were classified in terms of their ethnicities, namely Nguni and Sotho. Among the Nguni are the Zulus, Xhosas, Tsongas, Vendas, Swatis and Ndebeles and among the Sotho are the Pedis, Tswanas and Sotho-speaking people of the apartheid era. They were referred to as 'Blacks'. In the Employment Equity Act No. 55 of 1998, the term 'Black' refers to Africans, Coloureds and Indians. It should be noted that references used in this chapter might sometimes use the term 'Blacks' to mean non-Whites and also to mean 'Africans', interchangeably or Black African.

3 Model C schools were introduced in terms of the Education Affairs Act No. 70 of 1988 that entailed the transfer of ownership from the state to the school even though they remained state aided. These schools received largest per capita budgetary allocations. Entrenched existing privileges with the best facilities and highly qualified teachers (Gazette No. 16987 vol. 169, 14 February 1996 - Education White Paper 2). Note that the Model C-former white schools - referred to throughout the chapter - do not include the white private or independent schools as the focus is on public schooling system.

4 For detailed figures between 1953 and 1988 see F. Troup. Forbidden Pastures: Education Under Apartheid.

5 Eastern Cape Planning Commission, 2013. Diagnostic overview of the Eastern Cape Province: A resource document for the Vision 2030 Development Plan; http://www. itec.org.za/wp-content/uploads/2014/03/ECPC_DiagnosticOverview.pdf.

6 ECDE Annual Report.

7 http://www.itec.org.za/wp-content/uploads/2014/03/ECPC_DiagnosticOverview.pdf.

8 See Stats SA: Mid-year population estimates 2018, http://www.statssa.gov.za/ publications/P0302/P03022018.pdf, also ECDoE Long-Term Provincial Education Plan Incoporating Schools' Rationalisation, April 2019. 
9 In South Africa, an MEC is a member of the province's cabinet, who is responsible for a particular portfolio of services in the provinces. In this case, the district officials will recommend which schools should be closed to the education MEC for the Eastern Cape province.

10 As described by Simkins (2003) and Moyo (2017) to mean incongruence between the lived experience(practise) which illustrates how the work is done in relation to how it has been planned for or envisioned (policy), pointing to the need to capture the unofficial way of doing this, which can become a structural constraint.

11 That is they were still within the education system as they were not regularised because the South African School Act (SASA) Sections 33 and 12A empower the MEC to officially close schools and for EMIS to de-register them from the education system once processes in the Act are followed. The process to address the 310 schools was taken back to the districts, EMIS as a source of data confirmed that some schools were closed as far back as 2009 .

12 https://www.provincialgovernment.co.za/department_annual/131/2014-eastern-capeeducation-annual-report.pdf.

13 https://docplayer.net/26053804-Eastern-cape-department-of-education-2016-17head-office-operational-plan.html.

\section{References}

Acemoglu, D., \& Robinson, J.A. (2006). Economic Origins of Dictatorship and Democracy. Cambridge: Cambridge University Press.

Andrews, M., Pritchett, L., \& Woolcock, M. (2017). Building State Capability: Evidence, Analysis, Action. Oxford: Oxford University Press.

Bardhan, P., \& Mookherjee, D. (Eds..) (2006). Decentralisation and Local Governance in Developing Countries: A Comparative Perspective. Cambridge:The MIT Press.

Battersby, J. (2004). Cape Town's Model C schools: Desegregated and desegregating spaces? Urban Forum. 15(3), 279-291.

Behr, A L. (1988). Education in South Africa: Origins, issues and trends, 1652-1988. Pretoria: Academia.

Bloch, G. (2010). Basic Education:Where Are We Going? Focus: The Journal of Helen Suzman Foundation, Issue 59, 10-16.

Booysen, S. (June 2001). Transitions and trends in policymaking in democratic South Africa. Journal of Public Administration, 36(2), 125-144.

Christie, P., \& Collins, C. (1984). Bantu education: Apartheid Ideology and Labour Reproduction. In: Kallaway, P. (Ed.), Apartheid and Education: The Education of Black South Africans. Johannesburg: Ravan Press.

Christie, P. (1985). The Right to Learn: The Struggle for Education in South Africa. Braamfontein: Ravan Press.

Cole, P. 2018. A Comparative Analysis of Education Expenditure. Eastern Cape Department of Education. Bhisho, Eastern Cape.

De Kadt,J. (2011). "Learner mobility in Johannesburg-Soweto, South Africa: dimensions and determinants," Ph.D. thesis, Wits School of Education, Faculty of Humanities, University of the Witwatersrand.

Dekker, E., \& Van Schalkwyk, O.J. (1996). Modern Education Styles. Johannesburg: Heinemann.

Fiske, E.B., \& Ladd, H.F. (2005). Racial equality in education: How far has south Africa come?. Working Paper Series SAN05-03, Duke:Terry Sanford Institute of Public Policy.

Government of South Africa. Constitution of the Republic of South Africa, 1996. Available at: https://www.gov.za/documents/constitution-republic-south-africa-1996 
Government of South Africa. Department of Basic Education. White Paper on Education and Training, 1995. Available at: https://www.education.gov.za/Portals/0/Documents/ Legislation/White\%20paper/White\%20paper\%20on\%20Education\%20and\%20 Training\%201995.pdf?ver=2008-03-05-111656-000

Eastern Cape Department of Education. ECDoE Annual Report 2015. Available at https:// provincialgovernment.co.za/department_annual/256/2015-eastern-cape-educationannual-report.pdf.

Government of South Africa. Department of Basic Education. 2011. Report on dropout and learner retention strategy to portfolio committee on education. Available at: http://www. education.gov.za/Resources/Reports.aspx

Government of South Africa. Directorate Rural Education. 2009. Guidelines for the rationalisation of small or non-viable schools. Available at: https://wcedonline.westerncape. gov.za/documents/LearnerPlacementPlans/DBE-Guidelines-for-rationalisation.pdf

Fedderke, J.W., de Kadt, R.H.J., \& Luiz, J.M. Uneducating South Africa: The failure to address the need for human capital. International Review of Education (UNESCO), 46 (3-4) (2000), 257-281. Working Paper No.2, Econometric Research Unit, University of the Witwatersrand.

Goodin, R., March, J., \& Olsen, J. (2013). The logic of appropriateness. In The Oxford Handbook of Political Science. Oxford: Oxford University Press.

Jacklin, H. (1994). Schooling in Gazankulu. Available at: http://wiredspace.wits.ac.za/ handle/10539/25649.

Jansen, J. D. (2004). Autonomy and accountability in the regulation of the teaching profession: A South African case study. Research Papers in Education, 19(1), 51-66.

Languille, S. 2018. 'Affordable' private schools in South Africa: Affordable for whom?. In P. Srivastava, and G. Walford (Eds.), Non-State Actors in Education in the Global South. New York: Taylor and Francis

Letsoalo, M. M. (1995). The Incorporation of the Seven Northern Transvaal Education Management Structures into a Provincial Unit. Thesis. University of the Witwatersrand.

Levy, B., Cameron, R., \& Naidoo, V. (2018). Context and Capability: A Tale of Two Bureaucracies. In Levy, Brian (Ed.); Cameron, Robert (Ed.); Hoadley, Ursula (Ed.); Naidoo, Vinothan (Ed.) (2018): The Politics and Governance of Basic Education: A Tale of Two South African Provinces. Oxford University Press, Oxford.

McIntyre, D., \& Klugman, B. (2003). The human face of decentralisation and integration of health services: Experience from South Africa. Reproductive Health Matters, 11(21), $108-119$.

Motala, E., \& Pampallis, J. (eds) (2002). The State, Education, and Equity in Post-apartheid South Africa:The Impact of State Policies. Burlington: Ashton Publishing.

Msila,V. (2009). School choice and intra-township migration: Black parents scrambling for quality education in South Africa. Journal of Education 46, 81-98.

Ncholo, P. (2000). Reforming the public service in South Africa: A policy framework. Public Administration and Development, (20),87-102.

Ngoma, W.Y. (2007). The Complexities of Organisational Change: The Case of the Eastern Cape Department of Education (Doctoral dissertation, University of Witwatersrand, 2007).

Nokwe, D. (1954). The Meaning of Bantu Education. Liberation. Digital Innovation South Africa. Available at: https://www.sahistory.org.za/archive/the-meaning-ofbantu-education

Ramoketsi, M.S. (2008). The transformation of Black school education in South Africa, 1950-1994: A historical perspective. Unpublished PhD Thesis, University of the Free State: Bloemfontein. 
Robinson, N. (22 February 2019). Are elite former white schools 'the best'? Mail \& Guardian. Available at: https://mg.co.za/article/2019-02-22-00-are-elite-former-whiteschools-the-best

Soudien, C. (2004). 'Constituting the class': An analysis of the process of 'integration' in South Africa. In L. Chisholm (Ed.), Changing Class. Education and Social Change in Postapartheid South Africa. Cape Town: HSRC.

Soudien, C. (2010). The reconstitution of privilege: Integration in former white schools in South Africa. Journal of Social Issues, 66(2), 352-366.

South African Institute of Race Relations, 1997. South Africa Survey 1996/97, Johannesburg. Sekete, G. P. I., Shilubane, M. S., \& Moila, B. M. (2001). Deracialisation and Migration of Learners in South African Schools. Cape Town: Human Sciences Research Council.

Thompson, L. M. (2000). A History of South Africa. London:Yale University Press.

Van der Berg, S. (2007). Apartheid's enduring legacy: Inequalities in education. Journal of African Economies, 16, 849-880.

Yee, A. S. (1997). Thick rationality and the missing "brute fact": The limits of rationalist incorporations of norms and ideas. The Journal of Politics 59(4): 1001-1039. 


\title{
13
}

\section{DOWNWARD SPIRAL OR \\ UPWARD TRAJECTORY?}

\section{Building a public profession to meet the shifting technical, social and political demands of education}

\author{
Joshua L. Glazer and Jal Mehta
}

\section{Acknowledgements}

The authors thank David Cohen, Megan Duff, Melanie Ehren, Michael Feuer, Penina Glazer, Diane Massell and Clarence Stone for their comments.

\section{Introduction}

The origin story of US public education differs notably from those of Europe and Asia, and these differences are critical to understanding how the US historically managed the issues of capacity, accountability and trust, as well as why this approach increasingly faltered in the latter part of the 20th century. In contrast to other countries where a formal system of education was an invention of the national government, in the United States, public education emerged largely as a grassroots movement motivated by local communities that saw economic and social returns in exchange for establishing and paying for a local system of schools. The combination of local governance and finance, secularism, and inclusiveness (for whites) facilitated a rapid expansion of schooling such that by the late 1800 s, the vast majority of white Americans were in publicly supported schools. The movement was supported by civic elites who saw public schools as a remedy for rising inequality, crime and social instability that they feared threatened to undermine the young republic (Kaestle, 1983).

By 1910, the movement had expanded to high schools, and by 1940 , nearly three quarters of American youth were enrolled in public high school (Kaestle, 1983). Though schools remained separated by race, African-Americans also made large gains in educational attainment during this period. A small segment of college-bound students studied in the 'honors track', but most students expected to learn the basics and graduate from high school, and the schools typically held 
up their end of that bargain. These may seem like modest aspirations by contemporary standards, but economists attribute America's economic dominance of the 20th century in large part to its mass education system that preceded those of Europe by over a generation (Goldin and Katz, 2009).

For our current purposes, what is important is that the social consensus that early reformers brokered in the late 19th century in which the public agreed to (a degree of) centralization, taxation and secularization held steady for a century (Kaestle, 1983). Modest goals, coupled with local control, ensured that problems of capacity, accountability and trust did not escalate out of control. Schools were expected to uphold American values, assimilate immigrants, provide custodial care for children and teach some basic academic content. Trust, in this context, was rooted in teachers and schools upholding American values; capacity inhered in the fact that those teachers embodied the values that they were expected to instill; and accountability was facilitated by a system of local control in which most consequential decisions (e.g. hiring) were made at the community level (Graham, 2005; Tyack, 1974). Most teachers had meagre knowledge of content and pedagogy, but by the standards of the time, little more than that was needed. Capacity, in other words, is relative to the task at hand, and the task of teaching was constructed in ways far less complex than contemporary standards.

The social contract inherent in this arrangement was expressed in what scholars have referred to as The One Best System. In The One Best System, local governments had a relative monopoly on the delivery of educational services; most educational decisions were made at the local level; and teaching, learning and administration were loosely coupled at every level of the system (Tyack, 1974). Structurally, The One Best System was characterized by the familiar look of US districts with a locally elected school board that hired a superintendent who, in turn, ran the central office. Schools were organized into elementary, middle and high schools; traditional subject areas; and grades. Few questioned or even imagined an alternative. ${ }^{1}$ Schools were not the only beneficiaries of public confidence, as Americans generally trusted government well into the second half of the 20th century (Goldin and Katz, 2009; Lipset and Schneider, 1987). The general point, however, is that the social contract between Americans and schools worked well so long as academic expectations were modest for the majority of students. As the last 50 years have shown this would not last.

\section{New goals, challenges to the social contract}

Midway through the 20th century, cracks appeared in the social contract that had maintained relative system-wide equilibrium. Accountability, capacity and trust, which for decades had been maintained on largely non-academic grounds, gradually shifted their locus to the question of academic achievement. This began in the 1950s, as critics pointed to lax academic standards in American schools (Bestor, 1953), a critique that was strengthened by the launch of Sputnik 
and the fear of losing the space race. These concerns were only accelerated by the events that followed.

Analytically, we can organize these events into four big shifts that motivated the press for increased academic accountability. The first was economic. The shift from an industrial to a post-industrial economy (Bell, 1973) made it increasingly difficult to live a middle-class existence without some post-secondary education, which increased the expectations for the K-12 system as a whole, as more students needed to be prepared for college. A second, and particularly monumental, shift came with respect to equity and race. Beginning with Brown v. Board (1954), continuing through the civil rights movements (the 1960s), state adequacy and equity lawsuits (the 1970s to present), and most recently the standards and accountability movement (1980s to present), the press for a more equitable school system has become a politically contentious issue that rests on the country's long history of racial inequality and oppression.

A third factor was international competition. The $A$ Nation at Risk report, published in 1983, argued in apocalyptic terms that American economic pre-eminence was at risk if its schools did not improve, which launched a flurry of school reform activities that continue unabated today. A final factor is decreasing confidence in the profession and the state - beginning in the 1960s with community control movements and continuing through today with the rise of market-based approaches to schooling as well as efforts to hold teachers and schools accountable for results (Confidence in institutions, 2017). Rising neoliberalism, pushed by a network of conservative actors and increasingly accepted by centre-left Democratic politicians, similarly embraced a framework that was less about distributive justice and addressing poverty and more about holding schools accountable for test score outcomes (Stone, 2019). At the same time, declining confidence in schools stretched across political lines. Conservatives saw urban districts as bloated, inefficient bureaucracies, whereas liberals were increasingly impatient with the slow pace of reform and the growing achievement gap (Henig, 2018).

All of these forces have put increasing pressure on what Meyer and Rowan (1977) called a 'logic of confidence' that traditionally prevailed in schools and replaced it with a much more sceptical 'logic of results' (Meyer and Rowan, 2006). The broad coalition that had supported the system of public schools for over a century began to wobble as primary stakeholder groups looked for new arrangements that they hoped would produce different outcomes and accommodate shifting ideological preferences. This growing dissatisfaction and commensurate lack of trust motivated policies with far-reaching implications for the education sector. Standards-based reform, stringent accountability and teacher-evaluation systems, the spread of charter schools ${ }^{2}$ and other market-oriented policies all challenged The One Best System that had characterized the great majority of US schools and districts for a century. The current system, with its eclectic variation of providers, networks and sub-systems, is substantially different, and even more fractured, from the one that existed for most of US history (Cohen et al., 2018). 
For traditional districts, then, the current period presents formidable challenges and significant risk. They educate the vast majority of US students, and a larger proportion of the most needy and difficult to educate (Rotberg, 2018). At the same time, they compete with charter schools and other sub-systems for teachers, students and other resources within a new political ecology that has shifted authority away from education's traditional power centres (Feuer, 2016; Henig, 2009; Reckhow, 2012). The spread of the Common Core State Standards $(\mathrm{CCSS})^{3}$ has set expectations at an all-time high while continued public scrutiny over performance, pressure from conservative groups seeking to weaken the legitimacy of the public system and declining public trust further exacerbate conditions under which many local leaders operate.

In sum, if capacity accountability and trust were once managed by the logic of confidence in which schools were expected to adhere to local norms and values, in the current era, public expectations for students' academic performance have greatly complicated the political, social and professional dynamics with which educational leaders must contend. This analysis raises the question that we turn to next: how do accountability, capacity and trust interact in this new environment?

\section{Capacity, accountability and trust: Resources for improvement or caught in a downward spiral?}

Systems thinking provides a useful lens to think about the US approach. Rather than assessing the impact of particular programmatic choices on short-term outcomes, a systems thinking approach examines how different elements of the overall ecology interact to produce differing outcomes. In particular, systems thinking identifies the importance of 'loops' - balancing loops, whereby actions provoke opposite reactions (such as how a thermostat kicks in cold air when it gets too hot), and self-reinforcing loops, whereby sets of actions tend to build on one another in an upward or downward spiral (Sweeney, 2016; Senge, 1990).

This framework offers multiple perspectives on the US system from which we sketch here two broad narratives. One is that myriad issues have thrust the system into a downward spiral of high accountability, low trust and low capacity. Underlying this interpretation is that beginning in the 1990s and culminating with the passage of No Child Left Behind (NCLB) in 2001, policymakers pursued an extensive effort to create a nationwide system of assessments and accountability without a similar effort to build the needed professional capacity, commitment and learning that might enable schools to achieve those ends. The choice of testing instruments was driven more by accountability concerns than learning - every child, in grades 3-8, in math and reading, every year - which led to the use of low-level off-the-shelf multiple-choice tests, relatively cheap and easy to administer and score, but not reflective of the complex thinking tasks desirable for 21st century schools (Yuan and Le, 2012). The combination of accountability radically outpacing support, and the chosen instruments emphasizing rote learning on multiple-choice tests created, by the end of the NCLB 
era, a reform approach that left many teachers 'demoralized' and politicians of both parties fleeing the legislation (Duff and Wohlsetter, 2019; Mehta, 2013; Santoro, 2018).

A second, more positive interpretation, argues that the standards and accountability movement exposed the limitations of the underlying educational infrastructure and, in doing so, stimulated important efforts at reform and improvement. The efforts of a handful of charter school networks, comprehensive school reform providers, as well as some high performing schools and districts can all be seen as evidence that the external shift in expectations created opportunities for educators, researchers and reformers to collaborate on important innovations and measurable changes in teaching and learning. This perspective also sees NCLB's requirement for disaggregated data as creating an unprecedented emphasis on equity, and an impetus to improve low-performing schools, and schools that cater to underserved populations. At least some analysts claimed that the policy succeeded in somewhat narrowing the achievement gap (Gamoran, 2007). In this version, the movement from No Child Left Behind era tests to Common Core-aligned assessments signals a welcome shift towards higher order thinking tasks, while the shift from NCLB to the more open 2015 Every Student Succeeds Act reflects gradual system-level learning about the limits of top-down reform (Duff and Wohlsetter, 2019).

Even such an optimistic interpretation, however, does not change the fact that political and social expectations have far outpaced the underlying educational infrastructure needed to achieve them, or that this infrastructure is just beginning to emerge. American schooling has long lacked a strong technical core to guide practice; teacher preparation is distributed across more than 1300 institutions of variable quality; and race, class, and geography highly influence the distribution of learning opportunities. Moreover, the opportunities opened to women and people of colour by the civil rights movement reduced the pool of captive skilled labour on which education historically relied (Mehta, 2013). For these and other reasons, then, when expectations increased, the capacity to elevate educational performance was inconsistent and often weak; most schools and districts lacked the expertise, norms and social organization to work collectively towards common educational goals, let alone achieve them. To the degree there was system-level expertise it was mainly found among a handful of skilled teachers and some high quality schools, but there were few mechanisms to mobilize that knowledge for systemic improvement.

Not surprisingly, then, the imposition of external accountability on a highly unequal system produced highly unequal results. For many schools and districts that served the most disadvantaged students, where high staff turnover is common and internal coherence is lacking, external pressure often led to a variety of perverse consequences - narrowing the curriculum, focusing on the 'bubble kids' (those just below the proficiency cut-off), teaching to the test and, in a few cases, outright cheating (Elmore 2004: Koretz, 2017). Accountability outpaced capacity and trust, frequently with unfortunate results. 
At the same time, other schools and districts reflected a happier marriage between capacity, accountability and trust. For example, Long Beach California, through the efforts of two long-standing superintendents, leveraged state standards to build a culture of learning, improvement and shared commitment to student outcomes that led to gradual improvement over time (Honan et al., 2004; Austin et al., 2006). Education leaders in Montgomery County, MD established a careful vertical alignment of standards along with thoughtful professional development and professional learning communities that enabled a cascade of positive changes over time (Childress et al., 2009). More recently, the Shelby County iZone has undertaken a multi-year, systemic effort to build professional capacity around an ambitious vision of teaching and learning in a highly impoverished community (Glazer, et al., 2020). In all these examples, system leaders incrementally built capacity (through extensive opportunities for professional learning), cultivated trust (through developing a culture of respect and learning and avoiding scapegoating) and balanced external accountability with internal accountability rooted in professional norms and common expectations for performance.

The examples are important in that they show how shifts in the political and social environment have the potential to serve as resources that enable leaders to design coherent, high-performing systems in which capacity, accountability and trust spiral upwards to the benefit of students, teachers and the public. At the same time, these types of success stories remain relatively rare. The question, then, is how can the United States capitalize on the few but meaningful examples of systemic improvement while avoiding the unintended and negative outcomes that threaten to erode trust and undermine capacity?

\section{Building a 'public profession'}

Teaching, perhaps more than any other occupation, is a profession whose practice is on public display and subject to social critique. Further, the public nature of the profession means that it is more sensitive to broader social dynamics than other professions - e.g. dentists, engineers, pilots and accountants - that can keep societal issues at arm's length. As noted earlier, politically and socially controversial issues such as race relations, international competition and economic equity shape public views and discourse about the teaching profession and its work. For education, then, a central question is how to maintain legitimacy in an increasingly polarized and distrustful society, and in a policy environment that is placing ever-increasing demands on it. ${ }^{4}$ In this section, we argue that a "public profession' - one that has the technical proficiency to cope with 21st century policy demands and is yet attentive to local values - is one way to manage this dilemma. We further contend that the key to accomplishing this is a partnership between the profession and the public. To elaborate this idea, we bring together sociological analysis of the professions with political science research on the nature of cross-sector coalitions that support social change. 
Professions engender public confidence when they conceptualize and reason about problems in ways that resonate with public values, and when they are perceived as solving the problems for which they claim authority (Abbott, 1988). Consider the example of alcoholism. Long recognized as a serious social problem, its treatment was initially the province of the clergy who saw faith as the cornerstone of effective treatment. But by the middle of the 20th century, the clergy lost authority to social workers whose conceptual and practical tools were better aligned with a society that had become less religious and more inclined towards scientific rationality. The victory of the social works was short-lived, however, as its failure to develop an effective remedy opened the door for doctors who conceptualized alcoholism as a disease amenable to medical treatment. Medicine has been vulnerable to the encroachment of lay groups (e.g. AA) that have proven equally effective (Abbott, 1988).

For education, thus, one part of the challenge is technical. In an era that demands and obsessively measures results, trust requires a far more technically proficient system of practice. Wide-scale technical proficiency, in turn, requires a commensurately coherent professional community. We say this because a profession's social legitimacy stems not from isolated cases of success, however inspiring they might be, but from the collective ability of the occupation to consistently deal with a set of social problems over which it claims authority. One key to collective efficacy is a shared set of professional tools such as curriculum, assessments, lesson plans and academic tasks. These are roughly akin to what standard contracts are to lawyers, the APGAR scale is to obstetricians, the $\mathrm{DSM}^{5}$ is to psychiatrists and the prayer book is to the clergy - the tools of the trade common to all who belong to its community of practice.

A second component is a shared theory of teaching and learning. Collective efficacy requires an accepted (even if partial) set of causal relationships that undergird a profession's problem-solving capacity. This can entail a scientifically validated set of causal relations, commonly held rules of thumb (Haas, 1993; Lampert and Graziani, 2009) or agreed upon relations between diagnosis and treatment. Shared theory is a key tool by which professions make sense of and reason about a domain of problems over which they claim to expertise (Abbott, 1988), provides a profession-wide 'schema' for the interpretation of experience ${ }^{6}$ (Håkanson, 2007); enables learning and incremental improvement within and across local contexts (Duguid, 2005; Glazer and Peurach, 2015); and supports problem-solving for novel cases (Patel, 1999). Shared theory is also what allows accountants, doctors, engineers, social workers and other professionals to diagnose, reason about and intervene on problems with a high degree of consistency across local and national contexts (Håkanson, 2007).

Teaching practice bound by shared tools and theory would represent a significant shift for a profession historically characterized by norms of privacy, suspicious of external intrusion into the classroom, and with little experience in collective problem-solving. As one long-time observer of the profession puts it, 'American teachers have historically treated instruction as if it were their 
personal possession'? Of course, this is not a historical curiosity, but a fundamental feature of a political system rooted in fear of central authority and designed to curtail it. It also is reflective of American social norms that have favoured a highly localized vision of education in which quality and legitimacy are rooted in local values as much as they are in universal conceptions of knowledge and learning. But as noted earlier, changes are afoot and have been for some time. Several traditional districts and some charter school networks have taken strides to deprivitise practice, build networks of schools organized around a shared technical culture and common tools, and establish the normative and organizational basis for meaningful collegial problem-solving (Cohen et al., 2017; Glazer et al., 2018; Johnson, 2019; Glazer, et al., 2020; Reinhorn, 2015). These changes to the social organization of schools and the concomitant shift from individual to collective conceptions of practice - if they continue to spread - have the potential to gradually strengthen education's legitimacy and engender public trust.

But a public profession cannot be built by teachers and school leaders alone. Creating the conditions for positive interactions between capacity, accountability and trust rests equally on the actions of policymakers and the public that elects them. Policies can be designed in ways that bring diverse constituencies together in support of common goals, or they can exacerbate social divisions in ways that erode trust and weaken possibilities for collective action. As we elaborate later, the United States is replete with examples of both.

In their landmark study of urban school reform, Stone et al. (1988) coined the phrase civic capacity to argue that broad-based, local coalitions are critical to sustaining public commitment to school improvement. Civic capacity, they argued, inheres in the joint commitments and shared understandings that bind key constituencies - e.g. the business sector, organized labour and civic elites into a single coalition in active support of ambitious reform which, in turn, can keep the pernicious effects of policy churn at bay. For example, states like Massachusetts and Kentucky which made early and sustained commitments to standards-based reform in the 1990s did so in part by building a diverse coalition of legislators, business leaders, state leaders, civil rights activists and other interest groups to support their respective reforms.

Conversely, policies that expose historic divisions by upending traditions of local control, replacing local (and often African-American) teachers and otherwise challenging traditional notions of schooling risk eroding trust and social legitimacy - particularly if they fail to deliver on promised results (Glazer and Egan, 2018). Examples of this dynamic can be found in Detroit, Newark, New Orleans and Memphis where new governance arrangements exacerbated historic grievances and consequently failed to develop trust and social legitimacy (e.g. Morel, 2018). For instance, an analysis into the political dynamics of a state takeover of schools in Memphis found that while state officials thought that the effort to improve local schools would engender community support, it instead surfaced longstanding tensions deeply embedded in the region's troubled history 
of race relations. As the analysis of one of the authors puts it, 'The formal link to Nashville, selection of a White superintendent from out of state, displacement of local teachers and administrators, loss of funds to the district, and use of non-local CMOs all, in their own way, stressed historic fault lines and further aroused mistrust and suspicion' (Glazer and Egan, 2018, 959).

The ideas behind civic capacity emphasize that education is as much a political enterprise as it is a technical one, and that building capacity and trust places demands on the public and its elected officials no less than it does on the profession. As Stone (2019) puts it, 'Good, bad, or mixed, politics is about how people cope with diversity in their interests in a world of interdependence' (2). Nowhere is this more true than education, and it is for precisely this reason that policies and reform initiatives, regardless of their intent and technical merits, have little chance of success if they do not bring together constituencies and stakeholder groups across the social and political spectrum. At the same time, politically inclusive and ideologically palatable policies do not necessarily produce meaningful changes in teaching and learning. A public profession must manage the politics of representation, the cultural sensibilities of local communities and the technical demands of service delivery.

\section{Conclusion}

Could such a partnership between the public and the profession take shape in the United States? A sceptic would have little trouble marshalling evidence against that possibility. A structurally fractured system and a politically divided society present formidable obstacles to both sides of the partnership. Unlike countries with more centralized systems, and in contrast to those professions that operate at a greater distance from government, many of the core tools of educational practice curriculum, assessment, interventions and professional development - are selected by local leaders from an unwieldy and notoriously uncoordinated collection of firms, non-profits and institutions of higher education that produce them. As such, no government office can legislate a professional community of teachers into existence, and no professional body can demand or enforce changes to practice.

Moreover, the increasing pluralism of the provider landscape further complicates the emergence of a profession committed to shared tools and theory. As we noted above, The One Best System is now one of many that also includes individual charter schools, charter networks, other types of networks, and private schools. Regardless of how one views these various arrangements, the practical implications are that teachers work in a diverse array of systems that differ in governance, educational philosophy, and organization. If a common technical orientation is to take hold among teachers, it will need to do so in a not-so-common array of work environments.

Further, writing in 2020, it is difficult not to conclude that the public side of the partnership faces equally daunting obstacles as historical divisions, the legacy 
of racism and segregation, rising inequality, xenophobia and political polarization make civic capacity an even more challenging proposition. Further, in the contemporary era, civic capacity entails more than brokering agreements in a single municipal context but rather encompasses multiple layers of government, the public and private sector, higher education, philanthropy, and numerous interest groups. Simplistic notions of 'district-led initiatives', 'philanthropic agendas' or 'federal priorities' belie the intersecting maze of organizations and agencies active in the education sector. Henig's (2009) depiction of this new localism 'as a more fluid and dynamic arena... in which education supporters must build cross-sector coalitions in order to hold their own' (127) aptly captures the challenge of civic capacity in the current era.

Despite these admittedly formidable, even daunting, challenges, we see reasons for optimism. One is that changes over the last several decades can be seen as evidence of system-level learning (though in a very American sort of way). As we noted above, the emergence of the CCSS, the national focus on equity (Peurach, 2019), the softening of norms of privacy among teachers (Johnson, 2019) and efforts to reduce financial inequality among districts would have been unimaginable as recently as the 1980s. It is true that these changes have not eradicated the education system's historic inequities or its extreme decentralization, or that many of the tools critical to a more coherent profession are developed in the private sector, but the last several decades do represent a national effort to address historic deficiencies in the education system and to grapple with the entailments of systemic improvement.

In the United States, this type of system-level learning occurs in piecemeal fashion, as ideas and knowledge slowly spread across traditional districts, charter schools and networks, and non-profit and the private sector. A variegated provider landscape may not create optimal conditions for cross-organizational learning (Groth et al., 2017), but it does create opportunities for leaders to learn and innovate in ways that can inform the larger system. A brief anecdote illustrates this point. In Memphis, a tense political environment pitted the state's takeover district (known as the Achievement School District) against the local district as the two fiercely competed for students, teachers, dollars and legitimacy. Although politically worlds apart, the two systems, both under enormous pressure to show results, converged towards the same curriculum, instructional philosophy and improvement strategy (Glazer et al., 2018; Larbi-Cherif et al., 2018). Knowledge can spread even in a politically contentious environment.

That being said, the degree of political divineness that the ASD engendered in Memphis can be avoided. Ideologically driven politics on the national level can mask localities' more practical approach towards brokering compromises and promoting a shared civic agenda (Henig and Stone, 2008; Lyon and Henig, 2019). For sure, historic divisions and political polarization make these coalitions fragile - but fragile is not impossible. James and Deborah Fallows' portrait of 
American towns and cities depicted remarkable instances of civic capacity that stand in stark contrast to the national political scene.

Even as national politics induces distrust and despair, most polls show rising faith in local governance... [T] his was true even in cities with significant racial and economic diversity, from Greenville to Fresno, Los Angeles, and San Bernardino. ...Across the country, millions of people in thousands of organizations are working toward common goals, generally without being aware of how many other people and organizations are striving toward the same end (Fallows, 2018).

We do not wish to downplay the structural challenges of the US system or the social challenges of the contemporary era. Rather, our aim is to emphasize the centrality of professional and system-level learning as integral to fostering positive interactions among capacity, accountability and trust. This includes educational providers learning to design systems that incrementally move towards greater intellectual rigor and equity; school leaders learning to construct and manage school organizations that foster collegiality and collective problem solving; and teachers developing and refining a shared set of pedagogical tools and theories on which student learning ultimately depends. This also includes policymakers and the public learning that improvement is as much political as it is technical, that regardless of their intentions, policies are not granted a clean historical slate (Stone, 2019), and that educational initiatives can exacerbate historical grievances as easily as they can bring diverse interest groups together.

The partnership we envision, and the public profession that will emerge from it, can continue to materialize, but it will do so slowly and intermittently, and in a manner distinct to American governance, politics and history. Moreover, as new ideas spread, they will take on the imprimatur of local communities that vary in culture, history and capability, and that at best work to satisfice problems more than to solve them. As a mechanism of improvement, this is both slow and inconsistent - slow because there are few formal mechanisms for knowledge diffusion and inconsistent for all the reasons we listed previously. This is far from a perfect recipe for change, but compared to many past attempts, it may be the most promising path forward.

The authors dedicate this chapter to the memory of David Cohen, mentor, colleague, and friend.

\section{Notes}

1 Not all Americans benefited equally from The One Best System. For most of the 20th century, African-Americans attended segregated schools with far fewer resources than white schools. Even following court-mandated desegregation, schooling remained highly unequal in terms of funding, non-fiscal resources, and outcomes. 
2 Charter schools are public schools of choice that are run by private organizations. Roughly akin to England's academy schools, charter schools are exempted from many regulations that constrain traditional schools, can draw students from outside neighbourhood boundaries, and compete for students, teachers, and other resources with traditional schools and other charters. More than 3 million students are currently enrolled in the charters.

3 Common Core State Standards are a set of voluntary performance standards and aligned assessments developed by a consortium of states and non-profit organizations. Their purpose is to establish a common set of rigorous standards that would replace NCLB-era standards which in many cases were of much lower quality. Forty-one states have adopted the CCSS, though in some cases, states have made modifications.

4 In the tradition of Weber, Parsons, and other sociologists, we use the term legitimacy to indicate the extent that an organization's practices and aims are socially accepted and taken for granted within a particular cultural context. Meyer and Scott's (1983) definition is particularly useful: '... the extent to which the array of established cultural accounts provide explanations for (an organization's) existence, functioning, and jurisdiction, and lack or deny alternatives (201)'.

5 The Diagnostic and Statistical Manual of Mental Disorders is widely known as the DSM.

6 Schema typically refers to the cognitive structures that enable individuals to assimilate new information, but some theorists use it as a way to conceptualize organizational learning.

7 David Cohen, 2019, personal communication.

\section{References}

Abbott, A. (1988). The system of the professions. Chicago: University of Chicago Press.

Austin, J. et al. (2006). Long beach unified district (A): Change that leads to improvement (19922002). Cambridge, MA: Public Education Leadership Project at Harvard University.

Bell, D. (1973). The coming of post-industrial society: A venture in social forecasting. New York, NY: Basic Books.

Bestor, A. (1953). Educational wastelands: The retreat from learning in our public schools. Urbana: University of Illinois Press.

Childress, S. et al. (2009). Leading for equity. Cambridge, MA: Harvard Education Press.

Duff, M., \& Wohlstetter, P. (2019). Negotiating Intergovernmental Relations Under ESSA. Educational Researcher, 0013189X19854365.

Elmore, R. F. (2004) School reform from the inside out: Policy, practice, and performance. Cambridge, MA: Harvard Education Press.

Fallows, J., \& Fallows, D. (2018). Our towns: A 100,000-mile journey into the heart of America. New York: Vintage.

Feuer, M. J. (2016). The rising price of objectivity: Philanthropy, government, and the future of education research. Cambridge, MA: Harvard Education Press.

Gamoran, A. (2007). Introduction: Can Standards-Based Reform Help Reduce the Poverty Gap in Education? In A. Gamoran (Ed.), Standards-Based Reform and the Poverty Gap: Lessons for "No Child Left Behind", Washington, DC: Brookings Institute.

Glazer, J. L., \& Peurach, D. J. (2015). Occupational control in education: The logic and leverage of epistemic communities. Harvard Educational Review, 85(2), 172-202.

Glazer, J. L., \& Egan, C. (2018). The ties that bind: Building civic capacity for the Tennessee Achievement School District. American Educational Research Journal, 55(5), 928-964.

Goldin, C. D., \& Katz, L. F. (2009). The race between education and technology. Cambridge, MA: Harvard University Press 
Goldin, C. D., \& Katz, L. F. (2009). The race between education and technology. Harvard University Press.

Graham, P. A.. (2005). Schooling America: How the public schools meet the nation's changing needs. New York; Oxford: Oxford University Press.

Håkanson, L. (2007). Creating knowledge: The power and logic of articulation. Industrial and Corporate Change, 16(1), 51-88.

Henig, J. R. (2018). Charter schools in a changing political landscape. In: I. C. Rotberg \& J. L. Glazer (Eds.), Choosing Charters: Better Schools or More Segregation, New York: Teachers College Press.

Henig, J. R. (2009), The politics of localism in an era of centralization, privatization, and choice. Yearbook of the National Society for the Study of Education, 108(1), 191-218.

Henig, J. R. \& Stone, C. N. (2008). Rethinking school reform:The distractions of dogma and the potential for a new politics of progressive pragmatism. American Journal of Education, 114(3).

Honan, J. et al. (2004). Long beach unified district (B): Working to sustain improvement (20022004). Cambridge, MA: Public Education Leadership Project at Harvard University.

Johnson, S. J. (2019). Where teachers thrive. Harvard Education Press.

Kaestle, C. F. (1983). Pillars of the republic: Common schools and American society, 1780-1860 (Vol. 154). New York: Macmillan.

Koretz, D. (2017). The testing charade: Pretending to make schools better. Chicago: University of Chicago Press.

Larbi-Cherif, A., Lenhoff, S.W., \& Glazer, J.L. (2018). Where's the playbook? Curriculum, infrastructure, and high school turnaround. In: C. Meyers \& M. Darwin (Eds.), School Turnaround in Secondary Schools Possibilities, Complexities, \& Sustainability. Information Age Publishing.

Lyon, M. A., \& Henig, J. R. (2019). Blurring lines? How locally based collaborations handle the redistribution/development tradeoff. Urban Affairs Review, 55(4), 1100-1124.

Lipset, S. M., \& Schneider, W. (1987). The confidence gap: Business, labor, and government in the public mind. New York: Free Press.

Mehta, J. 2013. The Allure of order: High hopes, dashed expectations and the troubled quest to remake American schooling. New York, NY: Oxford University Press.

Meyer, J. W. (1977). The effects of education as an institution. American Journal of Sociology, 83(1), 55-77.

Meyer, J., \& Rowan, B. (1977). Institutionalized organizations: Formal structure as myth and ceremony. American Journal of Sociology 83(1): 340-363.

Meyer, H.-D. (2006). The rise and decline of the common schools as an institution: Taking "myth and ceremony" seriously. In H. D. Meyer \& B. Rowan, The New Institutionalism in Education. Albany: State University of New York Press.

Meyer, H.-D., \& Rowan, B. (2006). The new institutionalism in education. Albany: State University of New York Press.

Morel, D. (2018). Takeover: Race, education, and American democracy. Oxford University Press.

Reckhow, S. (2012). Follow the money: How foundation dollars change public school politics. Oxford University Press.

Santoro, D. (2018). Demoralized: Why teachers leave the profession they love and how they can stay. Cambridge: Harvard Education Press.

Senge, P. (1990). The fifth discipline: The art and practice of the learning organization. New York, NY: Doubleday.

Stone, C. N. (2019). Rhetoric, reality, and politics: The neoliberal Cul-de-Sac in education. Urban Affairs Review. Online version downloaded from: journals.sagepub.com/home/uar. 
Sweeney, L. (2016). Systems thinking: A means to understand our complex world. https://asknature.org/resource/systems-thinking-a-means-to-understanding-ourcomplex-world/.

Tyack, D. (1974). The one best system: A history of American urban education. Cambridge, MA: Harvard University Press.

Yuan, K., \& Le, V. (2012). Estimating the percentage of students who were tested on cognitively demanding items through the state achievement tests. Santa Monica, CA: RAND Corporation. 


\section{4}

\section{TRUST, CAPACITY AND ACCOUNTABILITY}

\section{A triptych for improving learning outcomes}

\section{Melanie Ehren and Jacqueline Baxter}

\section{Trust, accountability and capacity for system improvement}

This book introduced trust and accountability and how both interact to improve the capacity of education systems to provide for high-quality and equitable education: How, like a classical triptych, the three variables must be considered, not in isolation, but in tandem, in order to offer a full and holistic system-wide perspective. We also positioned capacity as a precursor for both trust and accountability, making the trio of variables a dynamic one that changes over time. Accountability and trust are considered beneficial for schools and systems to have, or to build the capacity for change, but both can be at odds with one another: the direction of the relationship between trust and accountability is complex and bidirectional; high-stakes accountability often destroys trust or would be implemented in contexts where there is a pre-existing lack of trust in schools and teachers. But, on the other hand, accountability may also improve trust when locking partners into a sustainable collaboration. And to add to the complexity, capacity may serve as an outcome of both trust and accountability, such as when someone who has expert knowledge in a certain area is trusted to do his/her job well.

The intricacy of the relations between trust, accountability and capacity and how their interaction varies across countries and contexts is at the centre of this book. In this final chapter, we reflect on the lessons learned from the various countries presented in this book, whether and how trust and accountability improve education systems and how that depends on the institutional structures and the social and cultural context in which they operate. In this final chapter, we will also use the framework in Table 14.1 to understand some of the cross-country mechanisms and how trust and accountability operate to improve education systems or provide barriers for change. 
TABLE 14.1 Structural embeddedness of trust and accountability

\begin{tabular}{ll}
\hline System & Accountability systems: \\
& - Compliance oriented, professional and performance based \\
& General trust/distrust: \\
& - A predisposition to trust unknown others \\
& - Level of suspicion in society \\
Organization & - Internal accountability (e.g. quality assurance systems, school \\
& self-evaluation and quality culture/learning organization) \\
& Organizational/collective trust amongst school staff and with the \\
Interpersonal & - Accountability relations: the extent to which actors are held \\
relations & accountable for their behaviour and performance by other actors \\
& (through measures: inspections, testing, monitoring) and the \\
& consequences for low performance \\
& Interpersonal trust: willingness to take a risk based on an assessment \\
& of a trustee's competence, benevolence and integrity
\end{tabular}

The table presents accountability and trust on both the system level and organizational level, and as part of interpersonal relations. This distinction follows Lyon et al.'s (2015) argument that situational and contextual factors are important or even decisive to understand why people trust. We extend the argument by saying that the same applies to how individuals hold each other accountable. In some schools, teachers are working in silos without any accountability to peers or their head teachers, while in other schools, there is a strong professional culture where staff hold each other and are held accountable for high learning outcomes. The wider (accountability) system in which they work, as well as the general trust in the profession, will have an effect on such interactions as we will explain in this chapter.

\section{Interpersonal relations of trust and accountability}

Trust is a process that is deeply embedded in social relations (Lyon, 2005). In Chapter 1, we described trust as 'a trustor's willingness to take a risk based on assessments of a trustee's competence, benevolence and integrity' (Mayer et al., 1995; p. 156). We followed Oomsels and Bouckaert (2017, pp. 82-88) in explaining these three indicators of trust as follows:

- Competence: perceived ability, or expectation that the other party has competence to successfully complete its task.

- Benevolence: expectation that the other party cares about the trustor's interests and needs.

- Integrity: expectation that the other party will act in a just and fair way. 
This definition features interpersonal relations, such as between teachers in a school, or between teachers and head teachers: head teachers, for example, have to rely on their teachers' skills, knowledge, judgements or actions when they give them autonomy to decide on how to deliver the school curriculum and instruct their students. Trust between teachers is relevant as it allows them to share work-related or personal information of a sensitive nature (disclosure), have a collaborative school culture and, for example, work towards a well-aligned curriculum which allows students to transition from one grade to the next without unnecessary gaps in learning. Both elements - relying on someone else and disclosing information - are essential for trust. Gillespie (2015) argues that where there is no requirement to rely on someone else, there is also no need for trust.

Interpersonal relations often also have an element of accountability, such as in school inspector-head teacher relations, or when head teachers hold teachers accountable for student outcomes in staff performance reviews. We described such accountability relations as 'the extent to which actors are held accountable for their behaviour and performance by other actors, such as through inspections, testing, monitoring and review'. Such measures typically have formal and/or informal consequences in an accountability context: head teachers in England, for example, face additional inspection monitoring when their school is failing an inspection, while their careers often get sidetracked after a failing inspection. Teachers with below-average student outcomes might fail to receive their annual salary increment. These examples are of vertical accountability relations where individuals have a hierarchical positioning towards each other. Hooge et al. (2012) also argue for horizontal accountability between equal peers. Examples would come from professional accountability systems, such as the one in Finland, presented by Hwa in Chapter 11. In such systems, teachers typically peer review each other's work and observe and evaluate each other's lessons, creating a strong professional culture in the school.

\section{Three perspectives on trust and accountability}

Although trust and accountability both sit at the individual level, they are not uncontested partners. Six discusses the relationship between trust and accountability in Chapter 2 and whether these can meaningfully be part of one and the same relation. She presents the two perspectives on trust and control which have been presented in the wider organizational management literature. Accountability, in her description, is positioned as a form of control, but both accountability and control are, she argues, about setting standards, gathering information and making judgements, possibly followed by interventions. 'Control', according to Six, is usually defined from the perspective of the controller, while accountability takes the position of the account giver. In our framework, we use the two somewhat interchangeably as they both can be seen as an attempt by partners in an exchange relation to address the vulnerabilities inherent in trust by producing relevant information on someone else's competencies and intentions. 
Whether the production of accountability information and subsequent consequences destroys or improves trust is what is discussed in the two (complementary and substation) perspectives, presented by Six in Chapter 2. We add a third 'inverse perspective' here which seems particularly relevant for an education context where there is not always an option to opt-out of a relationship and where relationships, given the nature of education as a public good, are not necessarily transactional in nature. In an inverse perspective, control is seen to violate the underpinning principle of trust and that you cannot control someone you trust. Williamson (1991) and Gundlach and Cannon (2010), for example, argue that control stems from a position of distrust (Macaulay, 1963): if one is not trusted, one trusts less, leading to a lower level of trust in the relationship. In a school context, a teacher who is evaluated and observed by his/her head teacher might feel that he/she is not trusted to do his/her job, particularly when other teachers are not undergoing the same type of evaluation.

\section{Complementary perspective}

The first complementary perspective positions trust and control as complements which can reinforce one another and lead to better, and a broader set of performance outcomes (Barrera et al., 2015; Mills and Rubinstein Reiss, 2017; Näslund and Hallström, 2017). This perspective contends that governance of exchange relations may be crafted with multiple mechanisms that address different governance problems. In this perspective, the information gathered on someone's performance may, for example, confirm initial (positive) assumptions of someone's (perceived) trustworthiness and enhance the collaboration and trust between partners. Monitoring and control can also provide information that both partners can use to improve their exchange. In this case, control and monitoring and being accountable to someone else will (when implemented and enacted in a fair and just way and introduced in a collaborative setting) ensure that trust becomes a social reality, or an established feature of the relationship. As Näslund and Hallström (2017) explain, formal control may promote trust when those being regulated perceive the monitoring and sanction/reward process as a sign of good intentions and benevolence on the part of the regulator and when they interpret the monitoring as a signal of interest and credible concern.

In a school setting, a head teacher who performance manages his/her teachers would come to trust these teachers when these performance reviews confirm that teachers are doing a good job and are trying to teach well. Teachers would equally come to trust their head teacher when they feel they are treated fairly and just and understand the performance management to be implemented in an attempt to help them improve their work. In this case, the control and monitoring by the head teacher and teachers being accountable to their head teacher can promote trust and ensure that it becomes an established feature of the relationship. Formal control may thus promote trust when those being regulated perceive the monitoring and sanction/reward process as a sign of good intentions 
and benevolence on the part of the regulator and when they interpret the monitoring as a signal of interest and credible concern.

In Chapter 3, Six' reference to self-determination theory (SDT) allows us to understand the complementary nature of trust and control more widely and how the wider accountability system shapes such relations. SDT explains how social context, and the extent to which individuals feel supported in their basic psychological needs, will affect their intrinsic motivation, performance and well-being. She argues that teachers and school leaders who experience accountability as enabling and supporting them in what they are trying to achieve will be intrinsically motivated to meet standards and improve their work in line with the external accountability framework. The role of motivation is positioned as a key condition for a positive outcome of accountability and control, and contrary to the types of systems that start with a position of distrust in schools and teachers and try to coerce or pressure teachers and schools into change. Such 'trust-based accountability' would engage professionals in the development and execution of formal control or accountability measures, support professionals' own initiatives and allow them to make their own choices and have some discretion over the interpretation of standards. It would also take the perspective of professionals into account in the interpretation of accountability information. Learning-oriented feedback, given within meaningful social relationships and accompanied by opportunities for professional development to improve on measured standards, is also relevant, according to Six. These conditions are all less likely in high-stakes, punitive systems where small errors or underperformance are punished without sufficient opportunity to improve and accountability is perceived to be unfair and unjust, or when the cultural context has distrust embedded systemically within it as Baxter reports in Chapter 4.

\section{Substitution perspective}

The second substitution perspective positions trust and control as two separate governance mechanisms: you either transact on the basis of trust or contractual agreements and verification. In this view, control mechanisms are viewed as redundant and inefficient when there is trust between partners. The higher transaction cost of control and monitoring are seen to lower performance, particularly when measured in terms of efficiency (Granovetter, 1985; Williamson 1991), as Yi Hwa reports on the Finnish system in Chapter 11.

The argument was initially developed in the context of economic transactions where buyers either trust their supplier to deliver good-quality goods or control suppliers to work according to contractual specifications. In an education context, a similar logic, however, applies when we position teachers or head teachers as the suppliers of education, where parents and students (or a central state on their behalf) are 'buying' the education. The Chilean voucher system, presented in Chapter 10, where schools get paid on the basis of student numbers and attendance, comes quite close to such a transactional relationship. When there 
is a high level of trust in the relationship, the need for effortful monitoring and frequent reanalysis of a situation or relationship is reduced as it enables people to make intuitive judgements and evaluations on the basis a few simpler rules or cues (Lewicki and Brinsfield, 2015; Ostrom, 2010). In a context of high trust where partners in the relationship share the same set of goals and are committed to meeting these goals, intensive monitoring is seen to be unnecessary and costly.

When we apply this logic to a schooling context, we would expect a head teacher not to performance manage his/her staff through a formal process of annual reviews. Trust would allow him/her to regularly observe his/her teachers, offer feedback on the quality of lessons and monitor in a more informal manner. More formal monitoring, in a substitution perspective, would be viewed as pointless, adding no extra value, and possibly detracting from the trust relationship already established between parties, particularly when there is clear goal commitment from both partners in the relationship.

Finland offers an interesting example of a system which embraces the substitution perspective. In Chapter 11, Hwa explains the absence of any form of formal accountability of teachers there and how external control is considered both unnecessary and undesirable. In her research, teachers explained how external control of teacher practice reduces the intrinsic motivation of teachers as their motivation comes from the freedom to 'use their skills and personalities to facilitate student learning as they see fit'. Such autonomy is thought to be incompatible with exhaustive standard-setting and information-gathering instruments, and also considered irrelevant given the high standards for entering the teaching profession. Intensive training ensures teachers' motivation and capacity to engage in effective practice and also legitimizes a system of high levels of teacher autonomy. Finland's high scores on PISA enhance such legitimacy.

\section{Inverse perspective}

The use of monitoring and control can, however, also crowd out trust in the sense that the level of trust in the relationship is eroded when control and monitoring are introduced. Control is thought to reduce the level of trust in the relationship when partners in a relationship attribute the collaborative behaviour to the existence of control and monitoring, instead of innate behaviours and values on which trust would be based. In our previous example of performance management in a school, the head teacher would believe that teachers are only doing a good job because they are performance managed instead of out of intrinsic interest and concern for students' learning and the quality of the school.

Those who argue for this inverse perspective (Gundlach and Cannon, 2010; Williamson, 1991) would state that control violates the underpinning principle of trust and that you cannot control someone you trust. These colleagues would argue that control stems from a position of distrust (Macaulay, 1963). If one is not trusted, one trusts less, leading to a lower level of trust in the relationship (Enzle and Anderson, 1993). 
The United States, presented by Glazer and Mehta in Chapter 13, offers an interesting example of how trust and accountability act as inverses on the system level. Their chapter describes how the declining trust in schools and the teaching profession in general provided the space for standards-based reform, stringent accountability and teacher evaluation systems, as well as the spread of charter schools and other market-oriented policies. The decline in trust, largely informed by a change in logic from confidence in schools to one of results, motivated the introduction of a series of policies which were all driven by a focus on student learning outcomes, intellectual rigour, and equity according to these authors. But rather than improving outcomes through strengthening the profession or the capacity of schools, high-stakes testing became one of the key levers to enforce change, with a further decline in public trust when these tests indicated failure or shifted teachers' allegiance to ensuring a high outcome on the test, instead of their students' individual learning and well-being or adhering to local norms and values.

Glazer and Mehta explore the potential contradiction between these two seemingly distinct values underpinning the teaching profession: teaching to/ incorporating local norms and values and thereby gaining local trust of parents and communities, or abiding by a set of national or state-level standards, enforced by external accountability which would perhaps engender more generalized trust in the education system as a whole. They argue that the divide can only be crossed when teaching becomes a 'public profession', one that is both universally competent and locally sensitive in combining technical expertise with a commitment to the needs of local communities. The converse also applies when institutions of accountability set up by the state, have no real legitimacy in the perceptions of those they would hold accountable: as reported in Chapter 4, systemic trust begins at government level, if corruption or nepotism is evident at this level within the system, it is very difficult to break down that culture and install trust at organizational level.

Their examples allow us to think about how, at the very core of where schooling takes place - through the work of teachers - a complementary perspective of trust and accountability can be established, through teachers who share ideas, language and tools in professional communities, build collective expertise and engage in joint problem solving, while similarly understanding that schools are more than 'achievement factories' and working with the local community to bring in the repositories of local culture and history, instil a source of local pride and take into account the unique, local needs. This, however, requires extraordinary capacities from both teachers and the local community in order to negotiate shared problem definitions and agree on solutions and, again, the trust to have those conversations and cross potential divides. It is also very difficult to achieve in cultures in which the 'achievement factory' ideal of education has unilaterally been accepted by government, such as in the case of England (Baxer and Floyd, 2019).

The US and Finnish examples also highlight how the initial starting point of how teachers are viewed by the public, their capacity to teach well and their sense 
of moral responsibilities and obligation matters in how control and accountability operate in relation to trust. Control, when introduced into a distrustful relationship, seems to escalate distrust, while the literature on external and internal motivation also indicates that external incentives to perform well may crowd out internal motives.

How control and accountability impact on trust also depends on the wider educational and societal context as our country chapters have shown. Countries vary in which types of control and monitoring are considered legitimate by the wider public as well as teachers, the types of instruments and measures they consider fair and adequate for measuring quality and outcomes and what is considered 'normal practice' and professionalism. These views also change over time as our chapters on the United States and Chile highlight, bringing in a temporal dimension of how trust and accountability interact over time to improve learning outcomes. Both levels of embeddedness (institutional and temporal) are further discussed below.

\section{Institutional embeddedness: Accountability systems and general trust}

The institutional context of trust is one that is often discussed in light of providing safeguards for trust, both on the organizational and system levels. Lyon (2005), for example, describes the political, legal and economic frameworks, as well as the informal rules that affect trust-building processes. These structures and informal rules affect the interaction between individuals and codify their practices as we explained in Chapter 2 when introducing three distinct types of governance of education systems (hierarchy, market and network) and how they regulate the interaction between educators and between educators and their stakeholders (parents, wider community).

In Chapter 12, Pakade and Chilenga-Butao also introduce the notion of uncodified practices: norms, conventions, self-imposed codes of conduct and shared understandings and expectations. They explain how these are shaped by, and inform system-level conceptualizations of trust, such as between different societal groups, public trust in the education system or in specific subsets of schools. Pakade and Chilenga-Butao illustrate this through South Africa's policy for closure of small, largely empty schools. The intention of South Africa's 'rationalization policy' was to amalgamate rural schools and thereby enhance available education resources, thus increasing the accessibility and efficiency of education in rural areas. The lack of coordination capacity and high levels of distrust between white and black bureaucrats, and between black bureaucrats from different Bantustans, however, meant that there was little follow-through in the planned closure, merger and realignment of schools, further reducing the overall capacity in the system when teachers follow learners to the new (merged) school while continuing to be on the staff roll of their previous school which is now empty but not yet closed formally. This 'double parking' means that there 
is an excess supply of teachers in the receiving school, where other schools have vacancies which cannot be filled. The lack of trust between government departments who work in silos reduces the coordinating capacity of the system and as result, the trust teachers and the public have in the education system to ensure a high-quality education. The example shows how local norms, values and distrust overrule national attempts to reform the education system for better learning outcomes.

Institutions - the formal and informal rules that organize social, political and economic relations (North, 1990) - affect the trust process through a logic of appropriateness, by socializing actors into widely accepted practice and influencing actors' conscious and unconscious decision to cooperate. Van der Voort 2017 argues that institutions play at least three roles in a trust relationship:

1. they may provide meaning to the circumstances before any relationship is built. When people are recruited into the roles of teachers and school principal, they will have an expectation on how to behave towards one another which will affect their relationship.

2. they influence the patterns of how trustors and trustees interact when they start to actively establish a relationship, such as how often teachers and their principal interact and the content of what they would communicate about, while

3. a trustor also has faith in the institutional arrangements themselves, making them the object of trust. A relevant example here would be of the fairness of established performance management systems within the school, or the external monitoring system by which teachers and principals are held to account.

Institutions therefore provide direction to the behaviour of trustors and trustees, whether consciously or not, both before and during cooperation. They can be positioned on the school organizational level, such as in the examples here, but equally on the system level where national systems of quality assurance structure such systems and interactions on the school level.

A good example of how institutions structure relations between teachers, principals and wider stakeholders comes from Chapter 7. Here, Altrichter describes how Austria's education system was traditionally characterized as state based with a high level of bureaucratic governance of schooling and compliance-oriented accountability. He describes how this system purposefully kept parents and other stakeholders out of the schooling exercise while organizing 'antagonistic cooperation' between the principal and agents in the education system: teachers and school administrators. He explains how the external accountability was geared towards input regulations (curriculum delivery and extracurricular activities) and to inform centralized decisions on resources, curricula and school structures. These set the conditions in which teachers do their work, but the technical core of their work (instruction, assessment and grouping of students in the classroom) 
was left untouched '... once the teacher closes the classroom door and starts teaching, the state cannot have a say in this area'. The examples Altrichter offers are of an accountability system which allows the various actors in the system to deliver on their own roles and responsibilities without much interaction and to have a culture where state administration and teachers were mutually and suspiciously observing each other, for possible infringements that may impact on them.

In Chapter 6, Ehren particularly reflects on trust of parents in the education system, how this is informed by the wider accountability of schools and is allowing for a high level of segregation. She describes the Dutch primary school system as one where students are concentrated in homogenous schools according to socio-economic background and level of parental education and income. Free school choice and a regulatory context of freedom of education provide the legitimacy for parents to choose a school on the basis of inner-group trust. The high level of trust in the education system allows them to be confident in choosing a school on the basis of similarity of peers, rather than the quality of education. Parents expect all schools to be of (similar) good quality and may, as a result, think that it does not matter where a child goes to school in terms of learning outcomes or entry to secondary education. The assumption is reinforced by the inspectorate's statutory framework and mission statement which emphasize its role in 'guaranteeing a minimum quality level across all schools'. Here, the high parental trust in institutional arrangements for school inspections are shaping interpersonal relations on the micro level and sadly not incentivizing interaction beyond one owns social/income group.

Interestingly, the lack of trust in the education system in South Africa also affects school choice patterns there, but in a somewhat different manner. Here, in the absence of trust in the wider system, trust in the quality of individual schools becomes one of the main indicators for school choice. In Chapter 11, Pakade and Chilenga-Butao talk about the high level of migration within South Africa as parents move their child to suburban former white, Indian and Coloured schools, which are seen to be more effective than black rural or township schools. Although there is little standardized accountability information available on the actual quality of these schools, they enjoy high levels of institutional trust of parents, which is informed by the visible stark differences in capacity and level of resourcing. Parents understand the quality of these schools as historically having the resources and status of providing better education in South Africa in terms of high-quality teaching, better discipline and safety. They offer 'a chance to better oneself through one's children' in providing their children with opportunities for entering the workforce parents did not have, and are, as such, a 'site for fast-tracking a way out of historical disadvantage'. This 'exit option' is, however, an expensive one for parents as it introduces a financial burden on the parent in payment of fees, transportation and other compulsory extracurricular activities. Given the overall high levels of poverty and unemployment in people's resident or indigent provinces, parents actively seek out better quality schools, leaving the schools in their home territories largely empty. 
At the system level, Kaye, in his chapter about the implementation of Edtech reforms in Kenya introduces another dimension of system-level conceptualizations of trust: teachers' trust in technology in general and the extent to which a particular tool can support their work and improve learning outcomes. In his Chapter 9, he explains how there was an initial level of high trust in educational technology amongst teachers, but how this did not mediate the successful implementation of a digital literacy programme. Kaye describes how the implementation of the programme was inadequately managed and monitored by the national government, and how this led to a widespread failure in implementation and wastage of financial resources. His chapter shows how the national government failed to take into account teachers' concerns with the technology, monitor implementation and subsequently build capacity for adequate roll-out and use, causing a widespread unwillingness and inability of teachers to use the technology.

Hwa, in Chapter 11, also points to the wider societal values and macrosystemic patterns in which education is set to explain how the public trust in a Ministry of Education and the teaching profession provides legitimacy for different types of teacher accountability and influences teacher motivation. She describes how the distinct approaches to teacher accountability in Singapore and Finland - a highly regulatory approach in Singapore versus a high level of teacher autonomy with initial standard setting in Finland - are both considered in legitimate in each country and, as such, both favourably influence teacher motivation and practice. In Finland, the high level of public trust in the education system and in teachers is informed by high standards at the point of entry into the profession; both allow for an accountability approach that gives teachers considerable autonomy over their daily work. The teaching profession in Finland has minimal rewards and penalties, with neither formal evaluation nor promotion. There are annual informal discussions with the head teachers and self-initiated collaborations and reviews and observations of lessons with colleagues, but these have no formal consequences or underpinning framework. According to Hwa, the admissions standards to the profession ensure that those who are chosen to enter the profession are highly motivated to teach well, while pre-service training ingrains the expertise that orients their motivation towards effective practices.

In Singapore, the public trust in the education system particularly operates through the high trust in the Ministry and is much more institutionally focused according to Hwa. The result is a teacher accountability system which allows for much less autonomy and has an extensive teacher performance management system incorporating detailed performance standards, regular formal appraisals, a career ladder and competition-based salary bonuses.

Particularly relevant to explain the high performance in both countries are, in her view society-wide affirmation of the value of education, a strong intrinsic motivation amongst teachers to teach well and engage in effective practice (instilled by high entry standards to the profession and a sense of moral 
responsibility and duty). She positions these in wider macrosystemic pattern, such as egalitarianism, stratification, autonomy and structure. A teacher who grew up in an egalitarian education system may well treat a norm-referenced performance bonus very differently from a colleague who was educated in a highly competitive and stratified system. Equally, a teacher who had been socialized to prize their professional autonomy might resent the introduction of detailed guidelines outlining their duties, whereas a teacher accustomed to relying on such guidelines might flounder if they were removed.

\section{The embeddedness of trust in the wider accountability system}

The notion of 'embeddedness of trust' emphasizes how we cannot understand 'trust' as a generic concept but rather as something that is context specific and varies according to the object or institution in which actors need to place their trust: the level of trust varies according to who needs to trust what. The examples above particularly indicate the relevancy of the wider accountability system in structuring these regulations. For example, in a compliance-oriented system, such as the one described by Altrichter for Austria's past system, the state typically introduces a set of regulations on school curriculum, assessment or targeted levels of student outcomes and expects schools to comply and meet these standards; control and monitoring are introduced to measure the level of compliance (e.g. through inspections). Here the accountability system would particularly regulate vertical relations and situate these in a hierarchy of command and control. These controls will have an effect on trust in such vertical relations, such as between teachers and head teachers (when external standards are incorporated in school performance management systems) and between head teachers and higher levels in the educational hierarchy (school boards, districts etc.). As Six argues in Chapter 3, trust and control can only complement each other in such relations when those held to account are involved in the setting of accountability standards and when accountability feedback is supportive and learner oriented.

In professional accountability systems, such as in Finland, relations are primarily horizontal and directed at how schools and teachers conduct their profession and/or at how schools and teachers provide multiple stakeholders with insight into their educational processes, decision-making, implementation and results. Hooge et al. (2012, p. 8) explain how such horizontal accountability is premised on the development and evaluation of shared expectations amongst students, teachers, school leaders and other local stakeholders about learning outcomes and service delivery. Peer review and school self-evaluation are typically part of horizontal accountability, informing relations within schools and between schools and their learners and parents. Trust is an important condition for such horizontal accountability to work as it allows unfamiliar actors to form a bridge and establish a 'world in common' and shared language to allow for a discussion about problems, weaknesses and ways to improve practice. 


\section{A temporal perspective: The dynamic nature of accountability and trust}

As mentioned earlier, trust relations are not static but shift over time as illustrated by diachronic investigations into the nature and scale of trust in societies, such as the longitudinal work done by sociologists such as Mead (Mead and Métraux, 2000). Priem and Weibel (2015, p. 271) talk about temporal embeddedness where past experience in a repeated game situation affects trustors' decisions. A good example of this is the case of teachers and head teachers who have a bad experience with a school inspector and who would be less likely to trust that same (or perhaps any other) inspector again in the future: transferring their poor experience with one individual, to categorization of the whole inspectorate as not worthy of their trust. When the experience oriented by the wider institutional structures in place, such as a high-stakes accountability system, the accountability exercise will likely be coloured by poor perceptions of the system as a whole: as culture is notoriously difficult to change, these feelings and perceptions will persist even when new arrangements are introduced.

Past experiences and understandings of accountability and control, as well as initial levels of trust, have an impact on educators' responses to changes, as we saw in Hwa's Chapter 11 about Finland: public trust in teachers greatly increased here after the 2001 release of PISA results. These results and Finland's exceptional performance conveyed the message that Finland's teachers were collectively doing a good job, and as a result silenced national debates about the need to introduce competition and higher levels of teacher accountability: Thus confirming the cyclical nature of trust, the 'trust breeds trust' argument, illustrated by Baxter, in Figure 4.3 of Chapter 4.

In Austria and Chile, educators' past experiences with governance structures and accountability arrangements shaped their perceptions of newly introduced arrangements for coordination and quality assurance of schools, as we saw in Chapters 7 and 10. Both countries tried to change their governance arrangements and ways in which schools are coordinated and held accountable, but attempts were reframed in ways that fit past experiences of teachers and school leaders and expectations of purposes of monitoring and evaluation: Austria, for example, tried to change from a compliance-oriented system towards one which aimed to engage schools in a cycle of self-evaluation and external target agreement. The change in system resulted from Austria's disappointing results in PISA and hoped to create a more flexible system that is able to allow for local adaptation and concentrate on the performance of student outcomes. It, however, required a substantial shift in roles of all stakeholders towards more negotiation and agreement, where teachers and school leaders were also expected to have the expertise and capacity to actively engage in school self-evaluation. Altrichter's chapter shows that such change requires substantial effort, particularly to overcome initial suspicion of the profession that feels that these agreements are introduced to control their work, rather than provide them with agency for change. 
In Chile, the 'New Publication Education' legislation introduced network governance as a new strategy for coordinating school quality and improvement. The law establishes Local Public Education Services which will progressively be introduced between 2018 and 2025 and replace municipalities as the intermediate level of the new system. These services will be in charge of new, mandated, networks of schools and are expected to lead the formation of these networks, coordinate school quality and ensure these networks support the improvement of schools. As Montecinos, González and Ehren explain, the past experience of educators under a highly hierarchical and marketized system, however, means that the networks that are currently being developed lack the intended horizontal structure and sets of relations and trust, needed for collaboration and improvement. The mindsets of school leaders, as well as those working at the management level of the network, are informed by past networks which primarily had a role in translating top-down policy to schools and explaining new government directives to schools. The high-stakes testing and free school choice of parents with a voucher system where school funding was tied to student attendance also mean that schools have been in competition with one another for almost three decades. These past experiences and entrenched ways of thinking and working feature in how schools engage with the networks, placing the prerogative of creating and leading networks exclusively at the local services level. The result is a highly hierarchically organized network with little genuine collaboration between schools in a territory. Without an active development of trust between the schools in the network and their capacity to collaborate in sharing problems and co-developing solutions, there will be limited collective action to improve performance of all schools in the territory. The fact that schools are also still held accountable for their individual school-level results further limits any meaningful collaboration and development of trust between network partners.

Both examples show how trust of professionals (teachers, head teachers) in their administrators (regional/national government) is an important condition for them to actively engage in improvement and monitoring, particularly where these systems require them to be open about the aspects of their (school's) functioning which need to be improved. Without such trust and an expectation that control and monitoring will restrict their professional autonomy or even cause harm, their involvement will be superficial at best.

In Chapter 8, Lee, Ho and Tay offer an interesting view on how such trust between stakeholders is built in Singapore. They talk about a system where the capacity of teachers and school leaders is continuously built within a national framework of a standardized curriculum and assessments where networks of school clusters provide the linking pin between the state and schools. Within these networks, teachers and head teachers (coordinated by a cluster superintendent) interpret and implement national policy collectively and are given the autonomy to make decisions on how to adapt national policy to their own context. Here the idea of a network is not understood as a means to communicate policy from the top-down (as in Chile and Austria), but as a vehicle for improvement. The wider 
context of Singapore, in terms of its collective culture, their structure for initial teacher training, but also their practice of rotating head teachers between schools every 6-8 years provides the conditions for these networks to act as levers for local capacity building, professional accountability and building of trust. As Lee and colleagues' chapter highlights, Singapore's culture is one which includes a broad commitment to a nation-building narrative of meritocratic achievement and social stratification, ethnic pluralism, and collective values and social cohesion. Parents, students, teachers and policymakers share a highly positive but rigorously instrumentalist view of the value of education at the individual level, and these shared values are reinforced through a single route for initial teacher training. The high level of trust in the profession and from the profession towards the state comes from a sense of collective responsibility for the performance of learners and the quality of all schools in the system, which is actively managed, such as through the rotation of school leaders. The fact that Singapore is a small state enables such close physical contact and frequent exchange, allowing educators across the system to collectively engage in policymaking, evaluation and improvement.

People trust each other and other institutions when these perform consistently according to expectations that fit their role. This requires people and institutions to have competencies and organizational capacity to perform according to what is expected. When that capacity is not there, external support needs to be provided. Accountability can trigger such support, and also set the standards/ define a shared set of norms around behaviour/performance, while also providing information about the of people and organizations, which supports them in bringing their performance/behaviour in line with agreed-upon norms. Expectations/norms need to be linked to conditions that promote school quality and good student outcomes for all. Those at a higher hierarchical level (leaders) set the example for behaviour further down the chain of command, while a crucial mass of peers engaging in a certain type of behaviour (e.g. corruption) create a critical mass or tipping point where others are coerced into engaging in similar behaviour.

A well-functioning bureaucracy (legal, political and economic) and existing organizational rules affect the degree and nature of trust and someone's ability to trust. Sitkin and Roth (1993), for example, explain how rules constrain and orient its members, while a well-functioning bureaucracy protects people from risk when engaging in new relationships. Having a clear set of rules stabilizes and regulates people's interaction and provides normative certainty and accountability of power, according to Oomsels and Bouckaert (2017). Rules and institutional templates enable people to enact their civil rights, while similarly enforcing duties and safeguarding autonomy, both individually as professionally. When there is a legal system to protect them, people will feel safe to suspend vulnerability and take risks in giving others the benefit of the doubt, and having an optimistic outlook for future interactions with people in general. For trust to flourish, it needs to be linked to the political context, as well as formal, 
political and legal institutions. Where this is absent, such as in cases in which corruption perceptions are endemic, the legitimacy of institutions whose purpose is to provide a framework that supports trust between they and the public they serve, it is very difficult to establish systemic and organizational systems that are based around trust. This, as Chapter 4 illustrates, can lead to chronic levels of undercapacity within education systems that are very hard to shift. Establishing a trusting culture at every level in the system is important, in order for it to thrive and be effective.

The chapters in this book have illustrated the complex relationship between trust, accountably and capacity and the relationship between this and raising learning outcomes in a number of country contexts. In bringing these three elements together in one volume, we have argued that for educational innovations to work well, it is vital to consider all three factors: a triptych in which each factor alone is meaningless, yet when considered as a whole, provides valuable insights into the reasons why learning outcomes improve, or fail to do so, in the complex and demanding operating contexts of today.

\section{References}

Barrera, D., Buskens, V., \& Raub, W. (2015). Embedded Trust: The Analytical Approach in Vignettes, Laboratory Experiments and Surveys. In: F. Lyon, G. Mőllering, \& M. Saunders (Eds.), Handbook of Research Methods on Trust. Cheltenham/Northampton: Edward Elgar Publishing.

Baxter,J., \& Floyd,A. (2019). Strategic narrative in multi-academy trusts in England: Principal drivers for expansion. British Educational Research Journal, 45(5), 1050-1071.

Enzle, M. E., \& Anderson, S. C. (1993). Surveillant intentions and intrinsic motivation. Journal of Personality and Social Psychology, 64(2), 257.

Gillespie, N. (2015). Survey Measures of Trust in Organizational Contexts: An Overview (pp. 225-240). In: F. Lyon, G. Möllering, \& M. Saunders (Eds.), Handbook of Research Methods on Trust. Cheltenham/Northampton: Edward Elgar Publishing.

Granovetter, M. (1985). Economic action and social structure: The problem of embeddedness. American Journal of Sociology, 91(3), 481-510.

Gundlach, G. T., \& Cannon, J. P. (2010). 'Trust but verify'? The performance implications of verification strategies in trusting relationships. Journal of the Academy of Marketing Science, 38(4), 399-417.

Hooge, E., Burns, T., \& Wilkoszewski, H. (2012). Looking Beyond the Numbers: Stakeholders and Multiple School Accountability, OECD Education Working Papers, No. 85. Paris: OECD Publishing.

Lewicki, R.J., \& Brinsfield, C. (2015).Trust Research: MeasuringTrust Beliefs and Behaviours (pp. 46-65). In: F. Lyon, G. Mőllering, \& M. Saunders (Eds.), Handbook of Research Methods on Trust. Cheltenham/Northampton: Edward Elgar Publishing.

Lyon, F. (2005). Managing co-operation: Trust and power in Ghanaian associations. Organization Studies, 27(1), 31-52.

Lyon, F., Mőllering, G., \& Saunders, M. N. K. (2015). Introduction. Researching Trust: The Ongoing Challenge of Matching Objectives and Methods (pp. 1-25). In: F. Lyon, G. Möllering, \& M. Saunders (Eds.), Handbook of Research Methods on Trust. Cheltenham/ Northampton: Edward Elgar Publishing. 
Macaulay, S. (1963). Non-contractual relations in business: A preliminary study. American Sociological Review, 55-67.

Mayer, R. C., Davis, J. H., \& Schoorman, F. D. (1995). An integrative model of organizational trust. Academy of management review, 20(3), 709-734.

Mead, M., \& Métraux, R. (2000). The Study of Culture at a Distance. Oxford, New York: Berghahn Books.

Mills, R.W., \& Rubinstein Reiss, D. (2017). The Role of Trust in the Regulation of Complex and High-Risk Industries: The Case of the U.S. Federal Aviation Administration's Voluntary Disclosure Programs (pp. 37-60). In: F. Six, \& K. Verhoest (Eds.), Trust in Regulatory Regimes. Cheltenham: Edward Elgar.

Näslund, L., \& Hallström, K.T. (2017). Being Everybody's Accomplice:Trust and Control in Eco-Labelling (pp. 145-181). In: F. Six, \& K.Verhoest (Eds.), Trust in Regulatory Regimes. Cheltenham: Edward Elgar.

North, D. (1990). Institutions, Institutional Change, and Economic Performance. New York, NY: Cambridge University.

Oomsels, P., \& Bouckaert, G. (2017). Interorganizational Trust in Flemish Public Administration: Comparing Trusted and Distrusted Interactions between Public Regulatees and Public Regulators (pp. 80-112). In: F. Six, \& K. Verhoest (Eds.), Trust in Regulatory Regimes Scoping the Field. The Netherlands, Amsterdam Google Books.

Ostrom, E. (2010). Beyond markets and states: Polycentric governance of complex economic systems. American Economic Review, 100(3), 641-672.

Priem, R. L., \& Weibel,A.A. (2015). Measuring the Decision to Trust Using Metric Conjoint Analysis (pp. 265-279). In: F. Lyon, G. Mőllering, \& M. Saunders (Eds.), Handbook of Research Methods on Trust. Cheltenham/Northampton: Edward Elgar Publishing.

Sitkin, S. B., \& Roth, N. L. (1993). Explaining the limited effectiveness of legalistic 'remedies' for trust/distrust. Organization Science, 4(3), 367-392.

Van derVoort, H. (2017). Trust and cooperation over the public-private divide: an empirical study on trust evolving in co-regulation. In Trust in Regulatory Regimes. Edward Elgar Publishing.

Williamson, O. E. (1991). Comparative economic organization: The analysis of discrete structural alternatives. Administrative Science Quarterly, 36, 289-296. 


\section{INDEX}

Note: Italic page numbers refer to figures.

academic outcomes 1, 42, 128, 131, 134, 167, 168, 170, 171, 173

accountability 2, 3, 15, 33, 49, 56, 62, 93, 107, 111, 119, 144, 145, 157, 159, 170,

191, 277, 288, 290; agents 104, 107, 111, 113, 115-119; dynamic nature, temporal perspective 300-303; governance reforms and relationship 144-153; information 291, 292; instruments 225 , 226, 228, 233-238, 240, 244, 245; inter-organisational trust for 115-119; interventions 9, 12; mechanisms 92, 191, 194, 196, 198, 235; of power 137, 302 ; relations $13,15,31,36,290$; relationships $13,63,267,268$; for school improvement 103; in schools, mesosystem 65-66; standards 37, 105, 106, 114, 299; structures 178, 197, 245; for system improvement 103-106; system trust for 111-115; trust, governance mechanisms 57-58; trust, relationship 56; trust and control, perspectives 58-59; see also individual entries accountability systems 19, 20, 61-63, 65, 66, 102, 103, 110, 112-115, 119, 295-299; embeddedness of trust 299; general trust and 295-299

Acemoglu, D. 260 achievement orientation 16,82 actors $11,12,24,32,36,48,57,66,67$, $72,81,83-85,91,93,94,149,195,214$
Ainscow, M. 35, 46

Aldridge, S. 31

Altinok, N. 185

Altrichter, H. 144

ambivalent attitude, professionalism 151 Andrews, M. 237, 269 annual teaching plan 34, 35 antagonistic cooperation 144-147, 296 apartheid education system 252, 254, 255, 259

Austrian education system 148, 153 autonomous motivation 60 autonomy 59; need for 63-64, 69, 70

Bachmann, R. 48, 51, 111-112, 118

Ball, S. J. 79

Bandura, A. 59

Banks, J. A. 140

Bantu Education 255, 256, 258

Bantustan education system 256, 257

Bantustans 253, 255-257, 259, 295

Barrera, D. 215

basic education system 254

Basic Laws Amendment Bill 95

basic skills 17, 82

Bax, E.H. 136

Baxter, J. A. 144

Bearak, J. M. 14

Bell, C. 131

benevolence 10, 12, 35, 42, 83, 84, 116, 237, 289, 291, 292

benevolence-based trust 117

Berry, J. 13, 14 
better learning outcomes 66, 119, 183, 197, 296

between-school coordination 147

between-school segregation 124-126, 138

Beugelsdijk, S. 19, 111

black schools 132, 141, 256, 257, 259, 262

Boterman, W. R. 124, 128-132

Bouckaert, G. 10, 289, 302

Bourdeieu, P. 87

Bovens, M. 12

Braithwaite, J. 68, 112

Brandsen, T. 23

Brauckmann, S. 14

Bray, M. 4

Brennan, J. 13

Brinsfield, C. 11

Bronfenbrenner, U. 4, 238, 254

Brown, C. 216

Brown v. Board 276

Bubb, S. 17, 18

Burns, T. 3, 49, 82, 90

Cannon, J. P. 291

capacity 82-83; development 118, 189, 190, 194; of educators respond to accountability 107-109; governance reforms and relationship 144-153; high level of 111, 258, 260, 261; for improvement 33, 45, 47, 119, 213; individual level 91-92; institutional level 92; as precursor to accountability 106-107; restoration of 90-96; society 94-96; system level 92-94; test developers and Inspectorates of Education 109-111; trust and distrust, education 83-85; see also individual entries

capacity building 164-168, 170-174, 176, 178, 195-197; in communities and networks 174-177; for standardisation and efficiency 166-168

Cerna, L. 11, 19, 239

charter schools 38, 276, 277, 283, 294

children 41, 42, 44, 45, 110, 126, 129 , 130, 132, 133, 138-141, 184, 258, 297

Chile 201-203, 205, 206, 210-212, 215, 217, 218, 300, 301

Chilean education system 202; coherent planning and systemic approach 204-205; governance of public education 204-205; heterogeneous capacities 205-206; hierarchical governance in 203-204; low accountability, municipal level 205; managerialism, coordination mechanism 207-212; market governance in 202-203; municipal public education, structural weaknesses 204; networking, coordination mechanism 212-217; new public education system 206-207

Chilean school system 203, 207, 217

Chilean voucher system 292

chronosystem 4, 5, 7, 9, 23-24

citizenship education 140, 174

civic capacity 281-284

classroom teaching 144, 145

climate of distrust 89, 90

Coffield, F. 2

Cole, S. 262

collective trust 16, 18, 41

comparative education 1

competence $10,12,35,55,59,64-66$, $69,83,84,151,289$; need for 64-65, 69,70

complementary perspective 291-292

compliance-oriented system 299, 300

control mechanisms 84, 149, 151, 292

cooperative atmosphere 17,82

corruption 78, 80-83, 86-90, 94-96, 257

cultures: distrusting 81, 82, 85, 86, 90, 96, 97; of trust 18, 91

curricular autonomy 148

Daly, A. 105

Darling-Hammond, L. 110

Das, T. K. 149

David, R. 82

De Charms, R. 59

Deci, E. L. 63, 65, 230

De Grauwe, A. 20, 33, 38, 39, 104, 105, 109

Dembélé, M. 109

Denton, D. S. 128

Digital Literacy Programme (DLP) 183, 189-191, 193-195, 197, 198; trust, capacity and accountability 193-194

distrust 85-86; cultures 86-90; high levels of 81, 86, 259, 295; overturning 90-96

diverse schools 126, 138, 139

Dougill, P. 13

Driessen, G. 135-136

Dronkers, J. 131, 136

Dutch education system 127-128, 138

Earley, P. 17-18, 22, 23, 38

Eastern Cape: interventions 261-262; PED 262-263

ecological systems theory 4, 5 
ecosystem 7, 8, 23, 245

Eddy-Spicer, D. 106, 108, 109, 114

EdTech, Kenya 188-198; Digital

Literacy Programme (DLP) 189-191;

trust, capacity and accountability

191-198; Tusome 190-191

educational leadership 17, 83

educational networks 44, 47

educational technology 182, 183, 185, 187, 189, 191, 195, 197, 298

education leaders 21, 279

education systems, governance $30-51$;

hierarchy 32-38

effective instructional arrangements 17, 83

egalitarian education system 245, 299

Ehren, M. C. M. 13, 37, 44, 47-49, 103-105, 108, 109, 114, 115, 118, 144

England 7, 8, 13, 45-47, 79, 102, 103, 107-110, 112, 152

Enhanced Performance Management System (EPMS) 224, 226-228, 230-232, 243

Eurostat survey 137

Evers, J. 72

evidence-based governance models 148-150, 153, 156; criticism and re-written versions of 150-153; PISA shock and 148-150

evidence-based strategy 150-152 exosystem 5, 7-9, 19-21, 55, 56, 61, 65, $66,72,254$; regulatory relation 66 ; trust and accountability in 66 extensive accountability instruments 232 , 243

external accountability 13, 15, 21, 24, 103, 105, 107, 108, 111, 214, 217, 294, 296

fairness 112-115

Ferris, J. 34

Finland 222-226, 232, 233, 236, 239-241, 244, 293, 298, 300

Finland's teacher accountability approach 242; see also teacher accountability

Fledderus, J. 111

Forsyth, P. B. 16, 105, 133

fragmented system 262, 263

Frank, D. H. 40, 41

freedom of education 127,128 , 138-140

Freidson, E. 11, 18, 20, 23, 33-35

frequent evaluation 17, 83

Fullan, R. 177
Gamoran, A. 126

general trust 136-138, 295-299

Gillespie, N. 11, 290

Glatter, R. 22

Godfrey, D. 7, 24, 47

Goldstein, H. 110

Goodall, C. 137

good education 23, 49, 72, 83, 84, 106, 119, 258

good governance 78,79

good-quality education 40, 205

Goodwin, A. L. 164

Government Technical and Advisory Centre (GTAC) 252, 254

Gray, A. 44, 47

Greany, T. 7, 22-23, 31, 38, 42, 45, 47-48, 216

Grek, S. 108-109

Grimaldi, E. 43, 45, 46

groups of schools 89, 93, 102, 128

groupthink 48, 51

Gundlach, G. T. 291

Gunningham, N. 112

Gutiérrez, G. 124, 125, 128, 138

Hadfield, M. 35, 46

Hall, C. 14, 108

Hallström, K. T. 15, 291

Handscomb, G. 24

Hardin, R. 37

Hargreaves, D. 177

Härkönen, U. 5

Harris, A. 110

Hatch, T. 20, 105

head teachers 7, 13-15, 47, 48, 116, 117, 289-293, 299, 301

Helderman, J. K. 216

Henig, J. R. 283

hierarchical education systems 32-38, 50, 176, 261; alignment and 36-38

hierarchical systems 23, 30, 33-35, 37, 38,50

hierarchical with network governance: broader educational outcomes 171-172; cluster of schools as structure 168-170; for school-led accountability and capacity building 168-172; school-led appraisal $170-172$

hierarchy 30-33, 35, 36, 43, 50, 51, 57, $58,66,72,201,244$

Higham, R. 7, 22, 31, 42, 45, 216

higher education 282, 283

high expectations 16,82

high general trust 126, 133, 138, 139 
high level of segregation 124, 125, 138, 297

high-quality education 23, 44, 104, 106, 111, 119, 214, 296

high schools 131, 274, 275

high-stakes accountability 8, 13, 24, 288

high-trust relations $12,31,37,50$

high workloads 109, 176

Höhne, T. 15

homogenous schools 129, 130, 132, 133, 138-140, 297

Honingh, M. 23

Hooge, E. 216

Hooge, E. 290, 299

horizontal accountability 21, 104, 207, 218, 290, 299

Hutchings, M. 47

Hutchinson, M. 109

IDB-LAPOP survey 215

inclusive education 44, 47, 48, 254

index of dissimilarity (DI) 129

individual level 82

individual schools 19, 21, 127, 147, 154,

155, 158, 210, 297

individual teachers 14, 18, 23, 227

initial teacher training 46, 245, 302

Inkpen, A. C. 111, 112

inner group identity 138

inner group trust 124-127, 129, 131, 133, 137, 139, 141

in-school coordination 147-148

institutional-based trust 111

institutional embeddedness 295-299

institutional level 82

institutional trust 112, 239, 241, 258, 297

integrity 11

inter-organisational trust 111, 115-117;

for effective accountability 115-119

interpersonal relations 10, 15, 19, 289-290

interpersonal trust 22, 35, 36, 80, 137, 239

interview methods 223

intrinsic motivation 55, 59-62, 64, 65, 69, 236, 238, 245

inverse perspective 293-295

Isaacs, S. 183

Jackson, D. 174

Jackson, J. 114, 115

Jaffer, K. 106

Jansen, J. D. 267

Jennings, J. 14

Jennings, J. L. 14, 105
Jerrim, J. 124, 125

Jessop, B. 51

Jiang, K. 4

Johnson, E. S. 7, 8

Kaffenberger, M. 183

Karsten, S. 131

Kelchtermans, G. 13

Kenis, P. 43

Kenya 182-191, 193-197

Kenyan education system 191, 194, 196, 198

Kenya's primary education system 183-188; EdTech, education quality 188-198; Kenyan development context 184-185; low learning outcomes 185-186; quality 187-188

Kim, J. 237

Klijn, E. J. 12

Klugman, B. 267

Kneyber, R. 72

Koppejan, J. F. M. 12

Koretz, D. M. 103

Köster, F. 49, 82, 90

Lasky, S. 108

Lave, J. 176

learning communities 172, 176

learning outcomes 37, 61, 81-83, 182, 183, 188, 189, 191, 196, 197, 288-303

Leckie, G. 110

legitimacy 112-115

Lemke, J. L. 24

Lerner, J. S. 237

level of trust 83, 171, 257, 261, 293, 299

Levy, B. 20, 21, 37, 261, 265

Lewicki, R. J. 11

local education councils 207, 215

logic of confidence 276

Looney, J. 34, 36, 105, 106

López, V. 217

low-performing schools 33, 47, 204, 206, 278

Luhmann, N. 118

Lyon, F. 289, 295

MacBeath, J. 41, 108

macro-level trust 111, 117, 238; see also system trust

macrosystem 4, 5, 7, 8, 21-24, 72, 223, 238, 244, 245

Makkai, T. 68

managerialism, coordination mechanism 207-212; evaluation and monitoring 
210; external evaluation, quality agency 210; internal monitoring, performance management 210 ; participation 211-212; planning 207-210; transparency, public reporting 210

Mansbridge, J. 115

Maritzen, N. 155

market-based systems 22, 38, 39, 50

market governance 38-43

market mechanisms 22, 38, 41-43, 50, 66,71

market models 201, 202, 204, 205, 216

Marquand Le E. L. 22

Massey, D. S. 128

Maxcy, B. 207

Mayne, J. 47

Mazibuko, S. P. 106

McEvily, B. 112

McIntyre, D. 267

Meadows, D. H. 4

Meier, B. 18

mesosystem 5, 6, 8, 9, 16-19, 56, 61, 62, $65,72,254$; accountability in schools 65-66; trust and accountability in 62-63

Meuleman, L. 22, 23, 43

Meyer, J. 276

microlevel 10-15

microsystem 4-8, 11, 21, 23

Mills, R. W. 215

monitoring systems 33, 106, 107

Moolenaar, N. M. 16

Morrone, A. 37, 137

Motala, E. 262

Mourshed, M. 2, 21, 22

Msila, V. 258

Muijs, D. 82

municipalities 129, 202, 204-206, 213, 227, 229, 232, 239, 244

Mwingirwa, L. M. 193

Näslund, L. 15, 291

national curriculum 33, 34, 169, 202, 204, 225, 232, 235, 236, 244

national education system 260

Nelson, R. 17, 18

Netherlands 124-141; parental preferences 130-132; perceived school quality in 130-132; religious and pedagogical profile 130-132; school choice 130-132; school population, composition 130-132 networked learning communities (NLCs) 172-174, 176, 177

network governance $31,43-51,57,72$, 168, 170, 177, 201

networking, coordination mechanism 212-217; alignment of goals 214-215; capacity building strategy 213 ; governance mode 212; participation 216-217 new education system 259, 260 new public management (NPM) 78, 79 new systemic quality management policy 153-156 non-operational schools 265

Noyes, A. 14, 108

O'Brien, S. 14

Ofsted 9, 114

The One Best System 275, 276, 282

O'Neill, O. 15

Oomsels, P. 10, 289, 302

orderly climate 17,82

Organisation for Economic Cooperation and Development (OECD) 82, 164

Ostrom, E. 94

Oviawe, J. 109

Ozga, J. 108-109

Pallas, A. M. 105

Pampallis, J. 262

parental education 127, 297

parental involvement 17,83

parental preferences $130-132$

parental school choice 38, 42, 127, 133

parents $3,10,12,19,39,42,43,84,95$, 116, 127, 130-134, 138, 141, 145, 297

Parker, P. D. 124

Pashiardis, P. 14

Pawson, R. 238

performance-based accountability 79, 110

performance grades 227, 229, 230, 232

performance management

system 226, 232

performance standard policy 148

Perryman, J. 8, 44, 47, 49

Persson, A. 96

Piezunka, A. 152

Pinochet, Augusto 202

Piper, B. 196

Plowright, D. 109

Poortman, C. L. 216

Porritt, V. 177

post-apartheid education system 253, 259-261

Priem, R. L. 300 
primary education 126-128, 132, 134, 254

primary schools $42,126,136$, 262, 264

Pritchett, L. 32, 33, 36, 37

private schools $127,130,136$, 214, 244, 282

private-subsidized schools 202-204, 217, 218

professional capacities 144, 149, 152, 153, 155-159, 277, 279

professional development 17, 83

professional learning 168, 170, 172-174, 176, 279

Programme for International Student Assessment (PISA) 1, 102, 149, 153, 156, 164, 222, 223, 231, 293, 300

Provan, K. G. 43

provincial education departments (PEDs) 252-254, 260-264, 266

psychological needs 59-60

public education 72, 201-204, 206, 209, 215, 217, 218, 274; system 22, 188, 212 public profession 279-282

public schools 127, 130, 136, 201, 202, 204, 211, 218, 256, 262, 274, 276

public trust 149, 151, 223, 224, 238, 240, 242-244, 294, 295, 298, 300

quality agency 203, 204, 210, 214, 215 quality development 20,21, 104, 154, 155 quality education 95, 102, 106, 118, 205, 259

rationalisation 253, 254, 261-267, 269

reformation 260

regulatee trust 68,69

regulatory relations: exosystem 66 ; trust in 68-69

regulatory trust triangle 66-69

reinforcing mechanism 132-133

relatedness 55, 59-61, 65, 66, 71; need for $65,69,70$

relationship of trust 12, 144, 145, 147, 149 , 152, 293, 296

re-written evidence-based policy: challenges and pitfalls 156-159

Robertson, S. L. 32, 34, 38, 39

Robinson, J.A. 260

Roth, N. L. 302

Rothstein, B. 19, 113, 114, 137

Rowan, B. 276

Rubinstein Reiss, D. 215

Ryan, R. M. 63, 65, 230
Sabelli, N. H. 24

Scheerens, J. 82, 88, 94, 126, 128

Schmidt, M. 14

Schneider, J. 104

school accountability 8, 166, 167, 174

school autonomy 21, 124, 125, 127, 129, 131, 133, 137, 139, 141, 147; policies 147; reforms 147-148

school-based curriculum 173, 177

school boards 3, 10, 12, 44, 47, 48, 105, 131, 188, 299

school choice 43, 50, 126-128, 130-132, 135, 138-140; Article 23 of the

Constitution 133-136; pillarized society 135-136; and trust in education system 133-138

school closures 265, 266

school cluster system 169, 169 , 170, 176, 177

school communities 16, 17, 95, 132, 174, 178, 210, 211

school curriculum 24, 290, 299

school evaluation 102, 170, 171

School Excellence Model (SEM) 170-172

school governing bodies (SGBs) 95, 259, 263-264

school improvement: accountability

for 103; plan 108, 203, 205,

209-211

school inspectors 79, 93, 119, 166, 290, 300

school leaders (SLs) 145-148, 150-152, 154-159

school leadership 147, 231

school-led accountability 165 , 168-170, 174

school-led capacity building: for innovations 172-174; networked learning communities across schools 173-174; professional learning communities (PLCs) 172-173

school networks 281, 301

school population 130, 131, 133

school principals 33, 155, 167, 168, 171, 209, 211, 214, 216, 218

school segregation 126-129

school self-evaluation 17, 103, 108, 155, 299, 300

Schools Rationalisation Project (SRP) 252-254, 261-265, 267-269; codified regulations for 263-265; uncodified practices in implementation 265-267 school systems 21, 92, 128, 148, 149, 151, 154, 156, 240, 243, 244 
Schulqualität Allgemeinbildung (SQA) 149, 153-159

Schunk, D. H. 234

Seashore Louis, K. S. 10

secondary education 102,126 , 134, 138, 297

secondary schools $23,157,167,223,228$, 256, 260, 264

segregation: institutional context of 127-130

Sekete, G. P. I. 258

self-determination theory (SDT) 55-56, 59-61, 63, 71-72, 292; core conceptual model of 61 ; intrinsic motivation and 59

self-improving school systems 176

self-improving system 177,178

self-segregated landscape 128-130

self-segregation 132-133

Shah, T. 13

Simola, H. 240

Sinclair, D. 112

Singapore 164, 166, 173, 176, 177, 222-224, 229, 232, 241, 242, 244, 245, 298, 302

Singapore education system 164-166, $171,174,177,239$; educational wastage and academic outcomes 167-168; evolution of 175 ; hierarchical governance 166-168; hierarchical with network governance 168-172; survival of nation, standardisation 166

Sitkin, S. B. 302

Six, F. 69, 85

Six, F. E. 11, 66, 69

SLEs 201, 206, 207, 209-218

small schools 254, 258, 262-264

Snyder, S. 4, 9

social contract $22,275-277$

societal level 82

Sohn, H. 14

Soudien, C. 262

South Africa 34, 35, 252-256, 258-260, 263, 295, 297

South African education system 253, 254; fragmentation and transition 254-257; fragmented trust, accountability and capacity, legacies 257-259

South African Schools Act 84 of 1996 (SASA) 263

South Africa's education system $253,257,260$
Southern and Eastern Africa Consortium for Monitoring Educational Quality

(SACMEQ) assessment 185, 186

special schools $44,45,51$

Spivack, M. 183

stability 144-147

stakeholder trust 195, 196

Stone, C. N. 281, 282

strong leadership 17, 83

structural embeddedness 289

student learning 3, 4, 11, 16, 17, 103, 108, 149, 158, 167, 172

Sturm, J. 135-136

substitution perspective 292-293

support schools 66, 103, 106, 186, 205, 213, 217

support teachers 62, 190, 194

Sustainable Development Goals

(SDGs) 182

system actors $83,93,153$

system capacity 83, 90, 93, 105, 197, 212

system change, preoccupation 1-4

system level 82

system-level improvement 78-97

system levels $82,83,85,89,92,97,155$, 156, 289, 294-296, 298

systems, defining 4-9

systems thinking 277

system trust 19, 111, 112, 117-119, 172;

for effective accountability 111-115;

fairness and legitimacy 112-115

Tacke, V. 15

teacher accountability 222, 223, 225, 232-234, 236, 238, 241, 245, 298, 300; comparable efficacy 233-239; Finland 239-241; in Finland and Singapore 224; generalised trust 239-241; information on teacher practice 226-229; institutional trust 241-243; instruments 223-225, 230, 232-234, 237, 238, 240, 243-245; instruments, compatibility 230-232; macro-level trust and 238-239; macrosystem and 236-238; rewards and penalties 229-230; Singapore 241-243; standards for teacher practice 225-226; teacher motivation and 234-238

teacher accountability systems 226 , 231, 298

teacher autonomy 48, 145, 231, 244, 293, 298

teacher motivation 223, 224, 234-238, 244, 298 
Teacher Performance Appraisal and Development (TPAD) 187, 188 teacher practice 224-227, 229, 231-235, 243, 244

teacher professionalism 36, 86, 147 teachers $11,12,15,40,63,65,72,145$, 157, 172-174, 176, 194, 224, 227, 230, 231, 237, 239; and principals 6, 22, 41, 42, 107, 216, 217, 296; and school leaders 7, 71, 106, 108, 114, 193, 281, 292, 300, 301; and schools 19, 22, 41, $56,145,153,156-158,174,244$

Teachers Service Commission (TSC) 186-188, 193, 194

teaching quality $13,63-65,108,149,195$, 225, 269

Temperley, J. 174

Teng, B. S. 149

Teo, C. H. 169

Tetlock, P. E. 237

Thinking Schools, Learning Nation (TSLN) vision 168, 169

Tilley, N. 238

Timperley, H. 18

transformation fatigue 267

transparency 78, 81, 195, 207, 210, 211, 215, 218, 261

trust: dynamic nature, temporal perspective 300-303; as enabler of effective accountability 111; governance reforms and relationship 144-153; school and system improvement 111; see also individual entries

trust-based accountability 55-73, 292; in education 71

trustworthiness $12,66,67,71,80,117$, 215, 216, 291

Tschannen-Moran, M. 11

Tudge, J. R. H. 8, 21

Tusome programme 190-191; trust, capacity and accountability 195-196

Tyler, T. R. 34
UNESCO report 88

UNICEF 205

unviable schools 252, 254, 259, 262, 263, 265

Uslaner, E. M. 37

value of education 41, 245, 298, 302

Van der Berg, S. 270

Van Der Slik, F. 135, 136

Van der Voort, H. 296

Van De Walle, S. 85

Van Ees, H. 11

Vanneste, B. S. 40-41

Veenstra, R. 131

Verhoest, K. 69

vertical accountability relations 12,290

Volante, L. 139

Waslander, S. 38, 39, 42

Weenink, D. 41

Weibel, A. 58

Weibel, A. A. 300

well-functioning bureaucracy 19, 302

well-functioning system 32, 95

Wenger, E. 176

White, R. W. 59

white elephant schools 258, 266

Whitty, G. 38, 41, 42

wider accountability system 299

Wilkins, C. 13

Williams, M. 216

Williamson, O. E. 291

Windzio, M. 30-32

World Development

Report 21

Yee, A. S. 252

Zeldenrijk, D. 130, 132, 139 